Semicontinuidade Inferior de Atratores para Problemas Parabólicos em Domínios Finos Ricardo Parreira da Silva 
SERVIÇO DE PÓS-GRADUAÇÃO DO ICMC-USP

Data de Depósito: 12/09/2007

Assinatura:

\title{
Semicontinuidade Inferior de Atratores para Problemas Parabólicos em Domínios Finos
}

\author{
Ricardo Parreira da Silva ${ }^{1}$ \\ Orientador: Prof. Dr. Alexandre Nolasco de Carvalho ${ }^{2}$ \\ Co-Orientador: Prof. Dr. José Maria Arrieta Algarra ${ }^{3}$
}

Tese apresentada ao Instituto de Ciências Matemáticas e de Computação - ICMC - USP, como parte dos requisitos para obtenção do título de Doutor em Matemática.

\author{
USP - São Carlos \\ Outubro/2007
}

\footnotetext{
${ }^{1}$ Suporte FAPESP $n .^{o}$ 03/12611 - 2, Suporte CAPES $n .^{\circ}$ BEX1190/05 - 9 .

${ }^{2}$ Universidade de São Paulo.

${ }^{3}$ Universidad Complutense de Madrid.
} 
À minha esposa, Karina. 



\section{Agradecimentos}

Não obstante sejam muitas as pessoas a quem devo agradecer, não posso deixar de atribuir especial merecimento a algumas delas.

Ao Prof. Dr. Alexandre Nolasco de Carvalho do ICMC-USP por sua amizade e orientação na execução deste trabalho.

Ao Prof. Dr. José Maria Arrieta Algarra da Universidad Complutense de Madrid por sua colaboração na execução de algumas etapas deste trabalho.

À minha família José Carlos, Ivone, Dirce, Juninho, Rodolfo e Gabrielzinho, cujo apoio incondicional permitiram que esta etapa de minha vida pudesse ser mais prazerosa.

E finalmente à Karina Schiabel, hoje Sra. Karina Schiabel Silva, esposa e companheira que conviveu, assistiu e participou da concepção desse trabalho. 



\section{Resumo}

Neste trabalho estudamos problemas de reação-difusão semilineares do tipo

$$
\begin{aligned}
u_{t}(x, t) & =\Delta u(x, t)+f(u(x, t)), & & x \in \Omega \\
\frac{\partial u}{\partial v}(x, t) & =0, & & x \in \partial \Omega .
\end{aligned}
$$

Desenvolvemos uma teoria abstrata para a obtenção da continuidade da dinâmica assintótica de $(P)$ sob perturbações singulares do domínio espacial $\Omega$ e aplicamos a uma série de exemplos dos assim chamados domínios finos. 



\section{Abstract}

In this work we study semilinear reaction-diffusion problems of the type

$$
\begin{aligned}
u_{t}(x, t) & =\Delta u(x, t)+f(u(x, t)), & & x \in \Omega \\
\frac{\partial u}{\partial v}(x, t) & =0, & & x \in \partial \Omega .
\end{aligned}
$$

We develop a abstract theory to obtain the continuity of the asymptotic dynamics of $(P)$ under singular perturbations of the spatial domain $\Omega$ and we apply that to many examples in thin domains. 



\section{Sumário}

Agradecimentos

Resumo

$\mathbf{V}$

Abstract $\quad$ vii

Introdução 1

1 Teoria abstrata dos sistemas dinâmicos dissipativos $\quad 7$

1.1 Semigrupos de operadores e equações de evolução . . . . . . . . . . . . . . . . 8

1.1.1 Potências fracionárias de operadores setoriais . . . . . . . . . . . . . . . . 11

1.1.2 Atratores para sistemas dissipativos . . . . . . . . . . . . . . . . 12

1.2 Grau Topológico . . . . . . . . . . . . . . . . . . . . . . . . . . 17

1.2.1 Grau Topológico em dimensão finita . . . . . . . . . . . . . . . . . . . . 17

1.2.2 Grau Topológico em dimensão infinita . . . . . . . . . . . . . . . . . . . . 19

2 Convergência compacta e continuidade dos atratores 23

2.1 Teoria linear . . . . . . . . . . . . . . . . . . . . . . . . . . . 24

2.1.1 Continuidade dos semigrupos lineares . . . . . . . . . . . . . . . . . . 31

2.1.2 Continuidade dos semigrupos não-lineares . . . . . . . . . . . . . . . 33

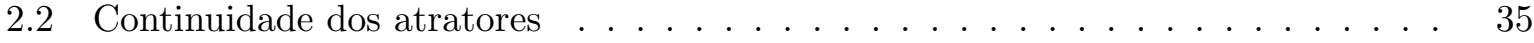

2.2 .1 Continuidade dos conjuntos de equilíbrio . . . . . . . . . . . . 36

2.2.2 Linearização em torno de um ponto de equilíbrio . . . . . . . . . . . . . . 39

2.2.3 Continuidade das variedades instáveis . . . . . . . . . . . . . . . . . . 44

2.2.4 Semicontinuidade inferior dos atratores . . . . . . . . . . . . . . 53

Segunda parte - Aplicações

3 Domínios finos - Perturbação simples $\quad 57$

4 Domínios finos curvos $\quad 69$

5 Domínios finos - Homogeneização da fronteira $\quad 77$ 
6 Domínios finos - Two layers 


\section{Introdução}

$\mathcal{S}$ eja em ciências físicas ou naturais, a compreensão de fenômenos evolutivos está intimamente ligada à sua previsibilidade ao longo do tempo. Quando da possibilidade de se determinar a evolução de seu estado inicial, é natural esperar, ao menos para sistemas que dissipam energia, que uma parcela considerável de sua dinâmica se concentre numa determinada região do seu espaço de fase. Esta região é conhecida como atrator.

Entretanto, os modelos sempre apresentam um certo grau de incerteza na determinação de seus parâmetros, de modo que se faz necessário determinar condições de estabilidade relativa a perturbações nos parâmetros do modelo proposto. Evidentemente os atratores também sofrem influência desta incerteza, e neste caso estuda-se quão robusto permanece o atrator. Em geral isto é feito comparando-se o "tamanho" dos atratores quando sob pequenas perturbações.

Aqui buscamos compreender o comportamento assintótico de sistemas de reação-difusão onde os parâmetros considerados são os domínios de suas variáveis espaciais que são singularmente perturbados. Como um protótipo consideremos $\Omega \subset \mathbb{R}^{n}$ um domínio limitado com certo grau de regularidade e seja $\Omega_{\varepsilon}:=\Omega \times(0, \varepsilon)$, para algum $\varepsilon>0$ pequeno. Em $\Omega_{\varepsilon}$ consideramos a equação do calor semilinear

$$
\begin{array}{ll}
u_{t}=\Delta u-u+f(u), & (0, \infty) \times \Omega_{\varepsilon} \\
\frac{\partial u}{\partial \eta_{\varepsilon}}=0, & (0, \infty) \times \partial \Omega_{\varepsilon} .
\end{array}
$$

Parametrizado por $\varepsilon$, o domínio $\Omega_{\varepsilon}$ varia de acordo com o parâmetro. Fazendo o parâmetro $\varepsilon \rightarrow 0$, analisamos a dinâmica de $\left(P_{\varepsilon}\right)$ comparando os atratores $\mathscr{A}_{\varepsilon}, \varepsilon \in(0, \bar{\varepsilon}]$, e $\mathscr{A}_{0}$, e damos condições suficientes para que a família de atratores $\left\{\mathscr{A}_{\varepsilon}\right\}_{\varepsilon \in[0, \bar{\varepsilon}]}$ seja contínua em $\varepsilon=0$.

Alguns dos primeiros a investigar este tipo de problema foram Hale e Raugel em [31]. Eles mostram a existência de uma equação limite quando $\varepsilon \rightarrow 0$ e resultados de semicontinuidade superior para a família de atratores, onde os domínios $\Omega_{\varepsilon}$ consistem da região delimitada pelo gráfico de uma função regular $g_{\varepsilon}: \mathbb{R}^{n} \rightarrow \mathbb{R}^{+}$. Neste mesmo espírito Prizzi e Rybakowski em [39] generalizam os resultados de Hale e Raugel considerando domínios mais gerais, podendo inclusive conter buracos. A correspondente equação limite é uma equação abstrata definida em um subespaço fechado $H_{s}^{1}(\Omega)$ de $H^{1}(\Omega)$. Além disso, para uma classe de domínios $\Omega \subset \mathbb{R}^{2}$, chamada de domínios bem decompostos os autores descrevem o problema limite explicitamente como um sistema de equações diferenciais ordinárias de segunda ordem definidas em um grafo e 
acopladas por certas condições de balanço.

Neste trabalho introduzimos um marco funcional para o tratamento de uma ampla classe de problemas singularmente perturbados que, em particular, inclui os domínios estudados em [31] e [39].

Em espaços funcionais apropriados, podemos escrever o problema $\left(P_{\varepsilon}\right), \varepsilon \in[0,1]$, como uma equação de evolução abstrata

$$
\left\{\begin{array}{l}
\dot{u}+A_{\varepsilon} u=F_{\varepsilon}(u), \quad t>0 \\
u(0)=u_{0} \in X_{\varepsilon}
\end{array}\right.
$$

para uma certa família de espaços $X_{\mathcal{\varepsilon}}$.

Como em [6], onde os autores fazem um cuidadoso estudo das propriedades espectrais de convergência do operador Laplaciano, nossa técnica tem como base um estudo criterioso da parte linear da equação $\left(P_{\varepsilon}\right)$ e esta informação é traduzida para sua dinâmica não linear. Primeiramente estudamos a convergência dos operadores resolventes $A_{\varepsilon}^{-1} \stackrel{\varepsilon \rightarrow 0}{\longrightarrow} A_{0}^{-1}$, em um sentido apropriado considerando o fato que os espaços $X_{\mathcal{\varepsilon}}$ variam conforme o parâmetro $\varepsilon$. Com a convergência dos operadores resolventes, mostramos um Teorema do tipo Trotter-Kato, isto é, mostramos a convergência $e^{-A_{\varepsilon} t} \rightarrow e^{-A_{0} t}$ dos semigrupos associados aos problemas lineares

$$
\left\{\begin{array}{l}
\dot{u}=-A_{\varepsilon} u, \quad t>0 \\
u(0)=u_{0} \in X_{\varepsilon}
\end{array}\right.
$$

quando $\varepsilon \rightarrow 0$.

Agora, se

$$
T_{\mathcal{\varepsilon}}(t)=e^{-A_{\varepsilon} t} u_{0}+\int_{0}^{t} e^{-A(s-t)} F\left(T_{\mathcal{\varepsilon}}(s)\right) d s
$$

é a solução de $\left(P_{\varepsilon}\right)$, com uma desigualdade do tipo Gronwall mostramos que $T_{\mathcal{\varepsilon}}(t) \stackrel{\varepsilon \rightarrow 0}{\longrightarrow} T_{0}(t)$, de modo apropriado. Destes resultados segue a semicontinuidade superior dos atratores $\mathscr{A}_{\varepsilon}$.

Nosso próximo passo é então escrever o problema estacionário,

$$
\left\{\begin{array}{l}
A_{\varepsilon} u=F_{\varepsilon}(u), \quad t>0 \\
u(0)=u_{0} \in X_{\varepsilon}
\end{array}\right.
$$

como um problema de ponto fixo $\left(u_{\varepsilon}=A_{\varepsilon}^{-1} F_{\varepsilon}\left(u_{\varepsilon}\right)\right)$. Usando a convergência $A_{\varepsilon}^{-1} \stackrel{\varepsilon \rightarrow 0}{\longrightarrow} A_{0}^{-1}$ mostramos a convergência dos equilíbrios do problema $\left(P_{\varepsilon}\right), \varepsilon>0$, para os equilíbrios do problema $\left(P_{0}\right)$. Além disso, se um equilíbrio $u_{0}^{*}$ de $\left(P_{0}\right)$ é hiperbólico, então $u_{0}^{*}$ é isolado e existe um único equilíbrio $u_{\varepsilon}^{*}, \varepsilon>0$, próximo a ele. Neste passo mostramos que $z_{\varepsilon}^{*}$ é um equilíbrio numa vizinhança de $u_{\mathcal{\varepsilon}}^{*}$ se, e somente se, $z_{\mathcal{\varepsilon}}^{*}=u_{\mathcal{\varepsilon}}^{*}+v_{\mathcal{\varepsilon}}^{*}$, onde $v_{\mathcal{\varepsilon}}^{*}$ é um ponto fixo do operador

$$
T v_{\varepsilon}=\left(A_{\varepsilon}-F_{\varepsilon}^{\prime}\left(u_{\varepsilon}^{*}\right)\right)^{-1}\left(F_{\varepsilon}\left(v_{\varepsilon}+u_{\varepsilon}^{*}\right)-F_{\varepsilon}\left(u_{\varepsilon}^{*}\right)-F_{\varepsilon}^{\prime}\left(u_{\varepsilon}^{*}\right) v_{\varepsilon}\right) .
$$

Assim, ao linearizarmos o lado direito da equação $\left(P_{\varepsilon}\right)$ ao redor de um ponto de equilíbrio, as convergências do operador resolvente e dos equilíbrios garantem a convergência do respectivo 
operador resolvente desta linearização. Adicionalmente, se o equilíbrio $u_{0}^{*}$ do problema $\left(P_{0}\right)$ é hiperbólico, então existe uma seqüencia de equilíbrios $u_{\varepsilon}^{*}$ do problema $\left(P_{\varepsilon}\right)$ tais que a variedade instável linear de $u_{\varepsilon}^{*}$ converge para a respectiva variedade instável linear de $u_{0}^{*}$.

Neste ponto assumimos que os equilíbrios do problema $\left(P_{0}\right)$ são todos hiperbólicos (propriedade genérica para o problema limite). Logo, a parte do espectro de $A_{\varepsilon}$ do lado direito do eixo imaginário consiste somente de um número finito de autovalores com multiplicidade finita. Se $Q_{\varepsilon}^{+}$é a projeção definida por essa parte do espectro de $A_{\varepsilon}$, temos, para $v_{0} \in Q_{\varepsilon}^{+} X_{\varepsilon}^{\frac{1}{2}}$ (que é finito dimensional), que a solução $v\left(t, v_{0}\right)$ de $\left(L_{\varepsilon}\right)$ existe para todo tempo negativo, e ainda $v\left(t, v_{0}\right) \rightarrow 0$ quando $t \rightarrow-\infty$. Quando perturbamos a equação $\left(L_{\varepsilon}\right)$ com uma não-linearidade $F_{\varepsilon}$ suficientemente pequena ${ }^{2}$, é natural então esperar que as soluções de $\left(P_{\varepsilon}\right)$ também existam para todo tempo negativo. Naturalmente, os dados iniciais para os quais essas soluções existem não podem estar distantes de $Q_{\varepsilon}^{+} X_{\varepsilon}^{\frac{1}{2}}$ mas sim em uma variedade (não-linear) próxima a ela. A técnica para se obter a continuidade dessas variedades é escrevê-las como um gráfico $W_{\varepsilon}^{u}=\left\{\left(z, S_{\varepsilon} z\right): z \in Q_{\varepsilon}^{+} X_{\varepsilon}^{\frac{1}{2}}\right\}$, e observar que as funções $S_{\varepsilon}: Q_{\varepsilon}^{+} X_{\varepsilon}^{\frac{1}{2}} \rightarrow\left(I-Q_{\varepsilon}^{+}\right) X_{\varepsilon}^{\frac{1}{2}}$ comportam-se continuamente com respeito ao parâmetro $\varepsilon$. A seguir damos uma idéia de como proceder para construir as funções $S_{\varepsilon}$.

Primeiramente decompomos o espaço $X_{\varepsilon}^{\frac{1}{2}}$ por meio da projeção $Q_{\varepsilon}^{+}$, isto é, $X_{\varepsilon}^{\frac{1}{2}}=Q_{\varepsilon}^{+} X_{\varepsilon}^{\frac{1}{2}} \oplus Q_{\varepsilon}^{-} X_{\varepsilon}^{\frac{1}{2}}$, onde $Q_{\varepsilon}^{-}=I-Q_{\varepsilon}^{+}$. Isto induz uma decomposição da equação $\left(P_{\varepsilon}\right)$ no seguinte sentido: se $z$ é uma solução de $\left(P_{\varepsilon}\right)$, escrevemos $z^{+}=Q_{\varepsilon}^{+} z$ e $z^{-}=Q_{\varepsilon}^{-} z$, e assim

$$
\begin{aligned}
& \dot{z}^{+}+A_{\varepsilon}^{+} z^{+}=H_{\varepsilon}\left(z^{+}, z^{-}\right), \\
& \dot{z}^{-}+A_{\varepsilon}^{-} z^{-}=G_{\varepsilon}\left(z^{+}, z^{-}\right),
\end{aligned}
$$

onde $A_{\varepsilon}^{ \pm}:=A_{\left.\varepsilon\right|_{Q_{\varepsilon}^{ \pm} X^{\frac{1}{2}}}}$, e $H_{\varepsilon}\left(z^{+}, z^{-}\right)=Q_{\varepsilon}^{+} F_{\varepsilon}\left(z^{+}+z^{-}\right), F_{\varepsilon} G_{\varepsilon}\left(z^{+}, z^{-}\right)=Q_{\varepsilon}^{-} F_{\varepsilon}\left(z^{+}+z^{-}\right)$são continuamente diferenciáveis com $H_{\varepsilon}(0,0)=G_{\varepsilon}(0,0)=0$ e $F_{\varepsilon}^{\prime}(0,0)=G_{\varepsilon}^{\prime}(0,0) \equiv 0$. Sendo $W_{\varepsilon}^{u}$ invariante, para um dado inicial $\left(\tau^{+}, S_{\varepsilon}\left(\tau^{+}\right)\right) \in W_{\varepsilon}^{u}$, a solução de $\left(D_{\varepsilon}\right)$ permanece no gráfico de $S_{\varepsilon}$ para todo tempo $t \in \mathbb{R}$. Isto nos diz que $z^{-}(t)=S_{\mathcal{\varepsilon}}\left(z^{+}(t)\right)$ e que, para todo $t$, a equação $\left(D_{\varepsilon}\right)$ se escreve como

$$
\begin{aligned}
& \dot{z}^{+}+A_{\varepsilon}^{+} z^{+}=H_{\varepsilon}\left(z^{+}, S_{\varepsilon}\left(z^{+}\right)\right) \\
& \dot{z}^{-}+A_{\varepsilon}^{-} z^{-}=G_{\varepsilon}\left(z^{+}, S_{\varepsilon}\left(z^{+}\right)\right) .
\end{aligned}
$$

Ainda, a solução $\left(z^{+}(t), z^{-}(t)\right)$ deve ir para 0 quando $t \rightarrow-\infty$ e, em particular, permanecer limitada para todo tempo negativo. Desde que

$$
z^{-}(t)=e^{-A_{\varepsilon}^{-}\left(t-t_{0}\right)} z^{-}\left(t_{0}\right)+\int_{t_{0}}^{t} e^{-A_{\varepsilon}^{-}(t-s)} G_{\varepsilon}\left(z^{+}(s), S_{\varepsilon}\left(z^{+}(s)\right)\right) d s
$$

fazendo $t_{0} \rightarrow-\infty$, obtemos

$$
z^{-}(t)=S_{\varepsilon}\left(z^{+}(t)\right)=\int_{-\infty}^{t} e^{-A_{\varepsilon}^{-}(t-s)} G_{\varepsilon}\left(z^{+}(s), S_{\varepsilon}\left(z^{+}(s)\right)\right) d s,
$$

\footnotetext{
${ }^{2}$ Supomos também que $F_{\mathcal{E}}(0)=0$ e $F^{\prime}(0) \equiv 0$
} 
e em particular

$$
S_{\mathcal{\varepsilon}}\left(z^{+}(\tau)\right)=z^{-}(\tau)=\int_{-\infty}^{\tau} e^{-A_{\varepsilon}^{-}(\tau-s)} G_{\varepsilon}\left(z^{+}(s), S_{\varepsilon}\left(z^{+}(s)\right)\right) d s .
$$

Assim, devemos ter $S_{\varepsilon}$ como um ponto fixo de um operador definido em um espaço de funções adequadas.

Uma vez obtida a existência das variedades instáveis como gráfico, a convergência das variedades instáveis lineares nos fornece a continuidade das variedades instáveis não-lineares. Finalmente, usando a estrutura gradiente dos problemas $\left(P_{\varepsilon}\right)$, mostramos a semicontinuidade inferior dos atratores e conseqüentemente sua continuidade.

Este roteiro está sendo empregado com sucesso no estudo da dinâmica assintótica sob perturbações singulares e já aparece de maneira implícita em [6, 13, 27].

Além dos problemas citados acima, há na literatura uma vasta gama de equações estudadas em domínios finos das mais diversas naturezas. Em [44] e suas referências podemos ter uma idéia das extensões que abrangem este tipo de perturbação. Para citar alguns exemplos: Antonci e Prizzi em [2] consideram o domínio $\Omega$ ilimitado, para Elsken em [22] o operador linear na equação $\left(P_{\varepsilon}\right)$ é um operador fortemente elíptico geral (não necessariamente auto-adjunto) e em [21] o domínio $\Omega$ consiste de faixas que se interceptam (L-shaped). Em [16] e [17], Ciuperca trabalha comprimindo com taxas distintas diferentes regiões do domínio $\Omega$, dando origem a problemas do tipo Dumbell $([3,7])$. Em vez de olhar diretamente o comportamento dos atratores, pode-se considerar isoladamente subconjuntos $B_{\varepsilon}(t)$ invariantes - objetos de estudo da teoria do índice de Conley - e resultados levando em conta domínios finos podem ser encontrados em Carbinatto e Rybakowski em [11]. Outros exemplos que poderão ser encontrados nesta tese:

Exemplo 1: Comprimimos como em [39] o domínio $\Omega$ por um fator $0<\varepsilon<1$ na direção vertical, obtendo o domínio comprimido $\Omega_{\varepsilon}$, isto é, colocando $\Omega_{\varepsilon}:=T_{\varepsilon}(\Omega)$, onde $T_{\varepsilon}: \mathbb{R}^{m+n} \rightarrow \mathbb{R}^{m+n}$ é a transformação $T_{\varepsilon}(x, y)=(x, \varepsilon y)$. Consideramos neste domínio a família de problemas de reaçãodifusão

$$
\begin{array}{ll}
u_{t}=\Delta u-u+f(u), & (0, \infty) \times \Omega_{\varepsilon} \\
\frac{\partial u}{\partial \eta_{\varepsilon}}=0, & (0, \infty) \times \partial \Omega_{\varepsilon},
\end{array}
$$

onde $\eta_{\varepsilon}$ é o vetor normal unitário exterior a $\partial \Omega_{\varepsilon}$, e $f: \mathbb{R} \rightarrow \mathbb{R}$ é uma não-linearidade satisfazendo certas condições de crescimento. Verificamos neste exemplo de forma detalhada que as hipóteses necessárias aos resultados abstratos estão satisfeitas.

Exemplo 2: Generalizamos o Exemplo 1 comprimindo como em [43] o domínio $\Omega \subset \mathbb{R}^{n}$ em uma variedade $\mathscr{M}$ de dimensão $m<n$.

Exemplo 3: Já neste exemplo estudamos o caso em que o domínio $\Omega$ se colapsa a um domínio de dimensão inferior tal que há uma região de sua fronteira que apresenta um comportamento 
altamente oscilante. Este problema está relacionado a problemas de homogeneização de fronteira $([4,48])$.

Exemplo 4: Este exemplo apresenta como em [46] um modelo de reação-difusão descrevendo o comportamento de um sistema com duas componentes finas adjacentes $\Omega_{1, \varepsilon}$ e $\Omega_{2, \varepsilon}$ separadas por uma membrana permeável $\Gamma$. Supondo que a interação dos reagentes ocorra somente na interface $\Gamma$, veremos que a intensidade da reação química em $\Gamma$ dependerá de quão fino forem os domínios preenchidos pelos reagentes.

$\mathrm{Na}$ análise de cada problema citado existe uma repetição de certos procedimentos. Como descrito, conseguimos dar um tratamento abstrato suficientemente satisfatório para os problemas envolvendo domínios finos listados na literatura. Com isso, o problema de se obter a continuidade dos atratores para essa classe de perturbações se resume a checar as hipóteses necessárias aos teoremas abstratos. Dessa forma esta tese está dividida da seguinte forma:

No Capítulo 1 coletamos uma série de resultados clássicos da Teoria de Semigrupos de Operadores Lineares e sua relação com a existência e unicidade de solução para o Problema de Cauchy; propriedades dos semifluxos $\left\{T_{\mathcal{\varepsilon}}(t) ; t \geqslant 0\right\}$ gerados por essas soluções de modo a garantir a existência de atratores e uma teoria de existência de pontos fixos para operadores compactos.

No Capítulo 2 apresentamos a teoria abstrata de convergência em espaços que variam e seguimos desenvolvendo resultados que garantam a continuidade dos atratores para os semifluxos $\left\{T_{\varepsilon}(t) ; t \geqslant 0\right\}$.

Nos capítulos subseqüentes aplicamos essa teoria abstrata aos Exemplos 1-4 descritos anteriormente. 



\title{
CaPÍtulo
}

1

\section{Teoria abstrata dos sistemas dinamicos} dissipativos

\begin{abstract}
$\mathcal{X}$
a tentativa de fazermos este texto tão auto-contido quanto possível, devotamos o primeiro capítulo a teoria dos Semigrupos de Operadores e sua relação com a existência de atratores globais para sistemas dissipativos. Expomos de forma rápida e sucinta um apanhado de definições e resultados que esperamos precisar nos capítulos subseqüentes. Observamos que algumas definições e resultados apresentados serão por conveniência enunciados na forma em que serão utilizados em nossas aplicações, e não em seu maior grau de generalidade. Tratando-se de ferramentas clássicas no estudo de equações de evolução em espaços abstratos, nos furtaremos a dar as provas da maioria dos resultados enunciados, podendo estas serem encontradas, por exemplo, em $[10,14,26,34,37,47]$.
\end{abstract}




\subsection{Semigrupos de operadores e equações de evolução}

Se $X$ e $Y$ são espaços de Banach sobre um corpo $\mathbb{K}(\mathbb{K}=\mathbb{R}$ ou $\mathbb{K}=\mathbb{C})$, denotamos por $\mathscr{L}(X, Y)$ o espaço dos operadores lineares e contínuos de $X$ em $Y$, munido da norma

$$
\|T\|_{\mathscr{L}(X, Y)}=\sup _{\substack{x \in X \\ x \neq 0}} \frac{\|T x\|_{Y}}{\|x\|_{X}}, \quad T \in \mathscr{L}(X, Y) .
$$

Em particular, se $X=Y$, escrevemos simplesmente $\mathscr{L}(X)$ para $\mathscr{L}(X, X)$. Por $X^{*}$ denotamos o dual topológico de $X$. A fim de fixarmos a notação, denotamos por $\langle\cdot, \cdot\rangle$ a $X-X^{*}$ dualidade e por $R(T)$ a imagem de $T$.

Definição 1.1. Um semigrupo de operadores lineares em $X$ é uma família a um parâmetro $\{T(t) ; t \geqslant 0\} \subset \mathscr{L}(X)$ tal que

(i) $T(t+s)=T(t) T(s)$, quaisquer que sejam $t, s \geqslant 0$,

(ii) $T(0)=I$,

onde I denota o operador identidade de X. Se, adicionalmente,

(iii) $\|T(t) x-x\|_{X} \rightarrow 0$ quando $t \rightarrow 0^{+}$, para todo $x \in X$,

dizemos que o semigrupo é fortemente contínuo, ou um $C^{0}$-semigrupo.

Teorema 1.2. Se $\{T(t) ; t \geqslant 0\} \subset \mathscr{L}(X)$ é um semigrupo fortemente contínuo em $X$, então existem constantes $M \geqslant 1$ e $\beta \in \mathbb{R}$ tais que

$$
\|T(t)\|_{\mathscr{L}(X)} \leqslant M \mathrm{e}^{\beta t}, \quad t \geqslant 0 .
$$

Definição 1.3. Seja $A: D(A) \subset X \rightarrow X$ um operador linear fechado. Ao conjunto $\left\{\lambda \in \mathbb{C}:(\lambda I-A)^{-1}: X \rightarrow X\right.$ existe e é limitado $\}$ chamamos o conjunto resolvente do operador $A$, e denotamos por $\rho(A)$. Ao conjunto $\sigma(A)=\mathbb{C} \backslash \rho(A)$ chamamos o espectro do operador $A$.

Definição 1.4. Se $\{T(t) ; t \geqslant 0\} \subset \mathscr{L}(X)$ é um semigrupo fortemente contínuo de operadores lineares, seu gerador infinitesimal é o operador definido por $A: D(A) \subset X \rightarrow X$, onde

$$
D(A)=\left\{x \in X: \exists \lim _{t \rightarrow 0^{+}} \frac{T(t) x-x}{t}\right\}, \quad A x=\lim _{t \rightarrow 0^{+}} \frac{T(t) x-x}{t} .
$$


O próximo resultado reúne algumas das propriedades dos semigrupos fortemente contínuos e seus respectivos geradores.

Teorema 1.5. Se $\{T(t) ; t \geqslant 0\} \subset \mathscr{L}(X)$ é um semigrupo fortemente contínuo de operadores lineares em $X$, então

(i) Para cada $x \in X, \lim _{t \rightarrow 0^{+}} \frac{1}{t} \int_{0}^{t} T(\tau) x d \tau=x$;

(ii) Para cada $x \in X$ fixado, a aplicação $t \mapsto T(t) x$ é contínua para todo $t \geqslant 0$;

(iii) A aplicação $t \mapsto\|T(t)\|_{\mathscr{L}(X)}$ é semicontínua inferiormente, e portanto mensurável;

(iv) Se A é o gerador infinitesimal de T(t), então A é um operador fechado e densamente definido. Além disso, para cada $x \in D(A)$, a aplicação $t \mapsto T(t) x$ é continuamente diferenciável com

$$
\frac{d}{d t} T(t) x=A T(t) x=T(t) A x, \quad t>0
$$

(v) Para cada $x \in X$ e $t>0$

$$
\int_{0}^{t} T(\tau) x d \tau \in D(A) \quad e \quad A \int_{0}^{t} T(\tau) x d \tau=T(t) x-x ;
$$

(vi) $\bigcap_{m \geqslant 1} D\left(A^{m}\right)$ é um subespaço denso de $X$;

(vii) Para $\operatorname{Re} \lambda>\beta$, onde $\beta$ é dado no Teorema $1.2, \lambda \in \rho(A) e$

$$
(\lambda-A)^{-1} x=\int_{0}^{\infty} \mathrm{e}^{-\lambda t} T(t) x d t, \quad x \in X .
$$

Teorema 1.6 (Hille-Yosida). Se $A: D(A) \subset X \rightarrow X$ é um operador linear, então as seguintes afirmações são equivalentes:

(i) A é o gerador infinitesimal de um semigrupo fortemente contínuo $\{T(t) ; t \geqslant 0\} \subset \mathscr{L}(X)$ tal que

$$
\|T(t)\|_{\mathscr{L}(X)} \leqslant M \mathrm{e}^{\beta t}, \quad t \geqslant 0
$$

(ii) A é um operador fechado, densamente definido, cujo conjunto resolvente contém propriamente o intervalo $(\beta, \infty)$ e

$$
\left\|(\lambda-A)^{-n}\right\|_{\mathscr{L}(X)} \leqslant M(\lambda-\beta)^{-n}, \quad \lambda>\beta
$$


Consideremos um espaço de Hilbert $H$, munido do produto interno $(\cdot, \cdot)$. Identificamos através do Teorema de Representação de Riez os espaços $H$ e $H^{*}$, e denotamos ambos por $H$.

Definição 1.7. Um operador $A: D(A) \subset H \rightarrow H$ é simétrico se $\overline{D(A)}=H$ e $A \subset A^{*}$, isto é, $(A x, y)=(x, A y)$ quaisquer que sejam $x, y \in D(A)$. O operador $A$ é auto-adjunto se $A=A^{*}$.

Lema 1.8. Seja $A: D(A) \subset H \rightarrow H$ um operador simétrico. Se $R(A)=H$ então $A$ é auto-adjunto.

Em muitos exemplos, a técnica empregada para verificar as estimativas espectrais necessárias para que um particular operador seja o gerador de um semigrupo fortemente contínuo são obtidas através da localização da chamada imagem numérica.

Definição 1.9. Se $A: D(A) \subset X \rightarrow X$ é um operador linear em um espaço de Banach complexo $X$, a sua imagem numérica $W(A)$ é o conjunto

$$
W(A)=\left\{\left\langle x^{*}, A x\right\rangle: x \in D(A),\|x\|_{X}=1, x^{*} \in X^{*},\left\|x^{*}\right\|_{X^{*}}=1,\left\langle x^{*}, x\right\rangle=1\right\} .
$$

No caso em que $X$ é um espaço de Hilbert, $W(A)=\left\{(A x, x): x \in D(A),\|x\|_{X}=1\right\}$.

Teorema 1.10. Sejam $A: D(A) \subset X \rightarrow X$ um operador fechado e densamente definido, $W(A)$ a imagem numérica de $A$ e $\Sigma$ um subconjunto aberto e conexo contido em $\mathbb{C} \backslash W(A)$. Se $\lambda \notin \overline{W(A)}$, então $\lambda-A$ é um operador injetivo com imagem fechada e, para todo $x \in X$,

$$
\|(\lambda-A) x\|_{\mathscr{L}(X)} \geqslant \operatorname{dist}(\lambda, W(A))\|x\|_{X} .
$$

Adicionalmente, se $\rho(A) \cap \Sigma \neq \emptyset$, então $\Sigma \subset \rho(A)$, e neste caso

$$
\left\|(\lambda-A)^{-1}\right\|_{\mathscr{L}(X)} \leqslant \frac{1}{\operatorname{dist}(\lambda, W(A))}, \quad \lambda \in \Sigma .
$$

Definição 1.11. Seja $\Delta_{\varphi}=\{z \in \mathbb{C}:|\arg \mathrm{z}|<\varphi\}$ e, para $z \in \Delta_{\varphi}$, seja $T(z)$ um operador linear limitado. A família a um parâmetro $\left\{T(z) ; z \in \Delta_{\varphi}\right\} \subset \mathscr{L}(X)$ é um semigrupo analítico sobre $\Delta_{\varphi}$ se

(i) A aplicação $z \rightarrow T(z)$ é analítica no setor $\Delta_{\varphi} \backslash 0$;

(ii) $T\left(z_{1}+z_{2}\right)=T\left(z_{1}\right) T\left(z_{2}\right)$, quaisquer que sejam $z_{1}, z_{2} \in \Delta_{\varphi}$;

(iii) $T(0)=I$;

(iv) $\lim _{\substack{z \rightarrow 0 \\ z \in \Delta_{\varphi}}} T(z) x=x$, para todo $x \in X$. 
Definição 1.12. Seja $A: D(A) \subset X \rightarrow X$ um operador fechado e densamente definido. Se existem constantes reais a, $M$ e $\varphi \in\left(0, \frac{\pi}{2}\right)$, tais que o setor $\Sigma_{a, \varphi}=\{\lambda \in \mathbb{C}: \varphi \leqslant|\arg (\lambda-a)| \leqslant \pi, \lambda \neq a\}$ esta contido propriamente em $\rho(A)$, e

$$
\left\|(\lambda-A)^{-1}\right\|_{\mathscr{L}(X)} \leqslant \frac{M}{|\lambda-a|}, \quad \lambda \in \Sigma_{a, \varphi}
$$

então o operador A é setorial do tipo $(a, M, \varphi)$.

Teorema 1.13. Se o operador $A: D(A) \subset X \rightarrow X$ é um operador setorial do tipo $(a, M, \varphi)$, então $-A$ é o gerador de um semigrupo fortemente contínuo $\{T(t) ; t \geqslant 0\} \subset \mathscr{L}(X)$, com

$$
T(t)=\frac{1}{2 \pi i} \int_{\Gamma_{a}} \mathrm{e}^{\lambda t}(\lambda+A)^{-1} d \lambda
$$

onde $\Gamma_{a}$ é a fronteira do conjunto $\{\lambda \in \mathbb{C}:|\arg (\lambda+a)| \leqslant \pi-\phi\} \backslash\{\lambda \in \mathbb{C}:|\lambda+a| \leqslant r\}$, com $0<\phi<$ $\varphi$ e $r$ pequeno, orientada no sentido da parte imaginária crescente. Adicionalmente, a aplicação $t \mapsto T(t)$ se estende analiticamente a uma função definida em $\left\{z \in \mathbb{C}:|\arg z|<\frac{\pi}{2}-\phi\right\}$ tomando valores em $\mathscr{L}(X)$ (ou a complexificação de $X$, se $X$ for um espaço de Banach real). Ainda, para $\operatorname{algum} K>0$,

$$
\|T(t)\|_{\mathscr{L}(X)} \leqslant K \mathrm{e}^{-a t}, t \geqslant 0, \quad e \quad\|A T(t)\|_{\mathscr{L}(X)} \leqslant K t^{-1} \mathrm{e}^{-a t}, t>0
$$

1.1.1 Potências fracionárias de operadores setoriais

Definição 1.14. Suponhamos que $A: D(A) \subset X \rightarrow X$ seja um operador setorial positivo, isto é, $A$ é setorial e $\operatorname{Re} \sigma(A)>0$, então, para todo $\alpha>0$ definimos

$$
A^{-\alpha}=\frac{1}{\Gamma(\alpha)} \int_{0}^{\infty} t^{\alpha-1} e^{-A t} d t
$$

$O$ operador potência fracionária $A^{\alpha}$, associado ao operador $A$, é a inversa de $A^{-\alpha}$, para $\alpha>0$, com $D\left(A^{\alpha}\right)=R\left(A^{-\alpha}\right)$. Definimos $A^{0}$ como o operador identidade em $X$.

Teorema 1.15. Se $A: D(A) \subset X \rightarrow X$ é um operador setorial positivo, então para todo $\alpha>0, A^{-\alpha}$ é um operador linear limitado em $X$, injetivo, e satisfaz $A^{-\alpha} A^{-\beta}=A^{-(\alpha+\beta)}$, quaisquer que sejam $\alpha>0, \beta>0$. Além disso, para $0<\alpha<1$,

$$
A^{-\alpha}=\frac{\operatorname{sen}(\pi \alpha)}{\pi} \int_{0}^{\infty} \lambda^{-\alpha}(\lambda+A)^{-1} d \lambda .
$$


Se A é um operador setorial positivo e $\alpha \geqslant 0$, então o espaço de potência fracionária $X^{\alpha}$ associado a $A$ é o espaço $D\left(A^{\alpha}\right)$ munido da norma do gráfico $\|x\|_{\alpha}=\left\|A^{\alpha} x\right\|_{X}, x \in X^{\alpha}$.

Teorema 1.16. Se $A: D(A) \subset X \rightarrow X$ é um operador setorial positivo, então $X^{\alpha}, \alpha \geqslant 0$, é um espaço de Banach e, para $\alpha \geqslant \beta \geqslant 0, X^{\alpha} \hookrightarrow X^{\beta}$. Além disso, se A tem resolvente compacto, então $X^{\alpha} \hookrightarrow X^{\beta}$ compactamente, para $\alpha>\beta \geqslant 0$.

Teorema 1.17. Seja $A: D(A) \subset X \rightarrow X$ um operador setorial com $\operatorname{Re} \sigma(\mathrm{A})>\delta>0$. Então para todo $\alpha \geqslant 0$, existe uma constante $C=C(\alpha)$ satisfazendo

$$
\left\|A^{\alpha} e^{-A t}\right\|_{\mathscr{L}(X)} \leqslant C t^{-\alpha} e^{-\delta t}, \quad t>0
$$

Teorema 1.18 (Desigualdade do Momento). Se $A: D(A) \subset X \rightarrow X$ é um operador setorial positivo, então para quaisquer $\beta<\alpha<\gamma$, se $x \in D\left(A^{\gamma}\right)$, existe uma constante $C=C(\beta, \alpha, \gamma)$ tal que

$$
\left\|A^{\alpha} x\right\|_{X} \leqslant C\left\|A^{\gamma} x\right\|_{X}^{\frac{\alpha-\beta}{\gamma-\beta}}\left\|A^{\beta} x\right\|_{X}^{\frac{\gamma-\alpha}{\gamma-\beta}}
$$

\subsubsection{Atratores para sistemas dissipativos}

Em nossas aplicações seguiremos utilizando resultados relacionados a existência global de soluções para equações de evolução semilineares em um espaço de Banach $X$. Nossas equações são escritas formalmente como

$$
\begin{aligned}
\dot{u}+A u & =F(u), \quad t>0 \\
u(0) & =u_{0},
\end{aligned}
$$

onde $A$ é um operador setorial em $X$, positivo, com resolvente compacto, $F: X^{\alpha} \rightarrow X$ é uma aplicação Lipschitz contínua e limitada para algum $\alpha \in[0,1)$ e $u_{0} \in X^{\alpha}$.

Definição 1.19. Se existem $\tau \in(0, \infty]$ e uma função $u \in C\left([0, \tau), X^{\alpha}\right)$ tal que $u \in C^{1}((0, \tau), X)$, $u(t) \in D(A)$, para todo $t \in(0, \tau)$ e a equação (1.4) se verifica para todo $t \in[0, \tau)$, então u é uma $X^{\alpha}$-solução da equação (1.4). 
Teorema 1.20. Para cada $u_{0} \in X^{\alpha}$ existe uma única $X^{\alpha}$-solução $u=u\left(\cdot, x_{0}\right)$ de (1.4) definida na semi-reta $[0, \infty)$.

Corolário 1.21. Para cada $\beta \in[\alpha, 1)$ e $u_{0} \in X^{\beta}$, existe uma única $X^{\beta}$-solução $u=u\left(\cdot, x_{0}\right)$ de (1.4) definida na semi-reta $[0, \infty)$.

A partir de agora, respaldados pelo Teorema 1.20, nos referiremos somente a soluções globalmente definidas $(\tau=\infty)$. Podemos ainda caracterizá-las e derivar algumas propriedades adicionais de regularidade.

Teorema 1.22. Uma função $u \in C\left([0, \infty), X^{\alpha}\right)$ é uma $X^{\alpha}$-solução de (1.4) se, e somente se,

$$
u(t)=e^{-A t} u_{0}+\int_{0}^{t} e^{-A(t-s)} F(u(s)) d s, \quad \text { para todo } t \geqslant 0 .
$$

Corolário 1.23. Se $u \in C\left([0, \infty), X^{\alpha}\right)$ é uma $X^{\alpha}$-solução de $(1.4)$, então $u \in C\left((0, \infty), X^{1}\right) \cap$ $C^{1}\left((0, \infty), X^{\gamma}\right)$ para todo $\gamma \in[0,1)$.

Teorema 1.24. Sejam $u \in C\left([0, \infty), X^{\alpha}\right)$ uma $X^{\alpha}$-solução de (1.4) e $\left\{u_{n}\right\}$ uma seqüencia em $X^{\alpha}$ com $u_{n} \rightarrow u_{0} \in X^{\alpha}$. Então, para todo $T \geqslant 0$,

$$
\lim _{n \rightarrow \infty} \sup _{[0, T]}\left\|u\left(t, u_{n}\right)-u\left(t, u_{0}\right)\right\|_{X^{\alpha}}=0 .
$$

Se $u=u\left(\cdot, u_{0}\right)$ é uma solução de $(1.4)$, podemos considerar a seguinte família de operadores $\{T(t) ; t \geqslant 0\}$, atuando em $X^{\alpha}$, definida por

$$
T(t) u_{0}:=u\left(t, u_{0}\right), \quad u_{0} \in X^{\alpha}, \quad t \geqslant 0
$$

Da unicidade e continuidade com relação aos dados iniciais das soluções da equação (1.4), podemos mostrar que a família de operadores $\{T(t) ; t \geqslant 0\}$ possui as propriedades

(i) $T(0)=I$;

(ii) $T(t) T(s)=T(t+s), t, s \geqslant 0$;

(iii) A aplicação $[0, \infty) \times X^{\alpha} \ni(t, x) \mapsto T(t) x \in X^{\alpha}$ é contínua; 
o que nos motiva a chamá-la um semigrupo fortemente contínuo (não-linear) associado a equação (1.4). Podemos ainda via Teorema 1.22, caracterizar os operadores

$$
T(t) u_{0}=e^{-A t} u_{0}+\int_{0}^{t} e^{-A(t-s)} F\left(T(s) u_{0}\right) d s, \quad u_{0} \in X^{\alpha} .
$$

Definição 1.25. Uma família $\left\{T(t): X^{\alpha} \rightarrow X^{\alpha} ; t \geqslant 0\right\}$ é um semigrupo fortemente contínuo se satisfaz as propriedades (i), (ii) e (iii) acima.

Definição 1.26. Se $\{T(t) ; t \geqslant 0\}$ é um semigrupo fortemente contínuo um subconjunto $B \subset X^{\alpha}$ é invariante (resp. positivamente invariante) sob $T(t)$ se $T(t) B=B, \forall t \geqslant 0(\operatorname{resp} . T(t) B \subset B, \forall t \geqslant 0)$. Um subconjunto $B \subset X^{\alpha}$ atrai $C \subset X^{\alpha}$ sob $T(t)$ se $\operatorname{dist}(T(t) C, B) \rightarrow 0$, quando $t \rightarrow \infty$.

Seja $B \subset X^{\alpha}$. Ao conjunto

$$
\gamma^{+}(B):=\bigcup_{t \geqslant 0} T(t) B
$$

chamamos a órbita positiva de $B$.

Se $u \in X^{\alpha} \quad$ e $H_{u}=\left\{\phi:(-\infty, 0] \rightarrow X^{\alpha}: \phi(0)=u, T(t) \phi(s)=\phi(t+s),-\infty<s \leqslant-t \leqslant 0\right\}, \quad o$ conjunto

$$
\gamma_{\phi}^{-}(u)=\bigcup_{t \geqslant 0} \phi(-t)
$$

onde $\phi \in H_{u}$, é chamado uma órbita negativa de $u$.

Uma órbita completa por $u \in X^{\alpha}$ é qualquer conjunto

$$
\gamma_{\phi}(u)=\gamma^{+}(u) \cup \gamma_{\phi}^{-}(u)
$$

Ao conjunto

$$
\omega(B):=\bigcap_{s \geqslant 0 t \geqslant s} T(t) B
$$

chamamos o $\omega$-limite de $B$.

Um subconjunto limitado $B \subset X^{\alpha}$ possui órbita positiva limitada se o conjunto $\gamma^{+}(B)$ é um subconjunto limitado de $X^{\alpha}$.

Observamos que a imagem $R(T(t))$ de $T(t)$ pode não ser todo o espaço $X^{\alpha}$. Logo, dado $u \in X^{\alpha}$, dizer que existe uma sua órbita negativa impõe certas restrições à escolha de $u$. O próximo lema dá uma caracterização para a existência de órbitas completas.

Lema 1.27. Um subconjunto $B \subset X^{\alpha}$ é invariante se, e somente se, para cada $u \in B$, existe uma órbita completa $\gamma_{\phi}(u) \subset B$. 
Definição 1.28. O semigrupo $\{T(t) ; t \geqslant 0\}$ é ponto dissipativo se existe um subconjunto limitado $B \subset X^{\alpha}$ que atrai todo ponto de $X^{\alpha}$ sob $T(t)$. O semigrupo $\{T(t) ; t \geqslant 0\}$ é assintoticamente suave se todo subconjunto não vazio, fechado, limitado e positivamente invariante, $B \subset X^{\alpha}$, contém um subconjunto não vazio $C \subset X^{\alpha}$ que o atrai.

Definição 1.29. Um subconjunto não vazio $\mathscr{A} \subset X^{\alpha}$ é um atrator global para o semigrupo $\{T(t) ; t \geqslant 0\}$ se $\mathscr{A}$ é compacto, invariante e atrai subconjuntos limitados de $X^{\alpha}$.

Observação 1.30. O atrator global, se existe, é único e maximal na classe dos subconjuntos limitados e invariantes de $X^{\alpha}$. Entretanto o atrator global é minimal na classe dos subconjuntos limitados de $X^{\alpha}$ que atraem limitados.

Teorema 1.31. Se o semigrupo $\{T(t) ; t \geqslant 0\}$ é ponto dissipativo, assintoticamente suave, e órbitas positivas de subconjuntos limitados são limitadas, então $T(t)$ possui um atrator global.

Com nossas hipóteses a respeito da equação (1.4), não é difícil checarmos a validade das condições do Teorema 1.31 para o semigrupo $\{T(t) ; t \geqslant 0\}$ dado em (1.5). De fato, da compacidade de $T(t)$ (derivada da compacidade do resolvente do operador linear $A$ ), segue que este semigrupo é assintoticamente suave. A dissipatividade pontual e limitação das órbitas positivas seguem dos lemas:

Lema 1.32. Se $B \subset X^{\alpha}$ é um conjunto limitado, então $\omega(B)$ é não vazio, compacto, invariante e atrai $B$. Além disso, se $B$ é conexo, então $\omega(B)$ é conexo.

Lema 1.33. Para cada conjunto limitado $B \subset X^{\alpha}$, existe um instante $\tau_{B}>0$, e uma constante $N_{B}$ tal que

$$
\sup _{t \geqslant \tau_{B}} \sup _{w \in T(t) B}\|w\|_{X^{\alpha}} \leqslant N_{B}
$$

Demonstração: Dados um conjunto limitado $B \subset X^{\alpha}$ e $u \in B$,

$$
T(t) u=e^{-A_{0} t} u+\int_{0}^{t} e^{-A_{0}(t-s)} F(T(s) u) d s .
$$

Das hipóteses sobre $F$ e $A$, existe uma constante $K$ tal que $\|F(w)\|_{X^{\alpha}} \leqslant K$, para todo $w \in B$ e

$$
\|T(t) u\|_{X^{\alpha}} \leqslant M e^{-\beta t}\|u\|_{X^{\alpha}}+M K \int_{0}^{t}(t-s)^{-\alpha} e^{-\beta(t-s)} d s .
$$

Como $B$ é limitado, existe $\tau_{B}$ tal que

$$
\|T(t) u\|_{X^{\alpha}} \leqslant 1+M K \beta^{-\alpha} \Gamma(1-\alpha)=: N_{B}, \quad t \geqslant \tau_{B} .
$$


Corolário 1.34. Se $B_{N}=\left\{u \in X^{\alpha}:\|u\|_{X^{\alpha}} \leqslant N\right\}$, então $\omega\left(B_{N}\right)$ é o atrator global para $T(t)$.

Demonstração: Claramente, $\omega(B)$ é compacto, invariante, conexo e atrai $B_{N}$. Notemos primeiramente que para todo conjunto limitado $B \subset X^{\alpha}$, existe $\tau_{B}$ tal que $T(t) B \subset B_{N}$, para todo $t \geqslant \tau_{B}$. Como $B_{N}$ é atraído por $\omega\left(B_{N}\right)$ e $B_{N}$ atrai $B$, o resultado segue.

Para certas classes de sistemas dinâmicos podemos descrever, com algum detalhe, a estrutura de seu atrator global.

Definição 1.35. Uma função contínua $V: X^{\alpha} \rightarrow \mathbb{R}$ é um funcional de Lyapunov para o semigrupo não-linear $\{T(t) ; t \geqslant 0\}$ se

(i) $V$ é limitada inferiormente e para cada $u \in X^{\alpha}$ a função $(0, \infty) \ni t \mapsto V(T(t) u) \in \mathbb{R}$ é não crescente,

(ii) $\mathscr{E}=\left\{u \in X^{\alpha}:-A u+F(u)=0\right\}=\left\{u \in X^{\alpha}: V(T(t) u)=V(u), \forall t \in \mathbb{R}^{+}\right\}$.

Se o semigrupo $\{T(t) ; t \geqslant 0\}$ possui um funcional de Lyapunov associado dizemos que o semigrupo possui estrutura gradiente.

Proposição 1.36. Se $\{T(t) ; t \geqslant 0\}$ possui estrutura gradiente e para um ponto $u \in X^{\alpha}$ sua órbita positiva $\gamma^{+}(u)$ é limitada, então $\omega(u) \subset \mathscr{E}$.

Teorema 1.37. Seja $\mathscr{A}$ o atrator global do semigrupo-não linear $\{T(t) ; t \geqslant 0\} . S e\{T(t) ; t \geqslant 0\}$ possui estrutura gradiente e o conjunto $\mathscr{E}$ é finito, então

$$
\mathscr{A}=\bigcup_{u^{*} \in \mathscr{E}}\left\{u \in X^{\alpha}: \exists \phi \in H_{u}, \phi(-t) \stackrel{t \rightarrow \infty}{\longrightarrow} u^{*}\right\}
$$

Definição 1.38. Seja $\Lambda$ um espaço topológico. Consideremos uma familia $\left\{J_{\lambda}\right\}_{\lambda \in \Lambda}$ de subconjuntos do espaço de Banach $X^{\alpha}$.

A família $\left\{J_{\lambda}\right\}_{\lambda \in \Lambda}$ é semicontínua superiormente em $\lambda=\lambda_{0}$ se $\lim _{\lambda \rightarrow \lambda_{0}} \operatorname{dist}\left(J_{\lambda}, J_{\lambda_{0}}\right)=0$.

A familia $\left\{J_{\lambda}\right\}_{\lambda \in \Lambda}$ é semicontínua inferiormente em $\lambda=\lambda_{0}$ se $\lim _{\lambda \rightarrow \lambda_{0}} \operatorname{dist}\left(J_{\lambda_{0}}, J_{\lambda}\right)=0$.

A familia $\left\{J_{\lambda}\right\}_{\lambda \in \Lambda}$ é contínua em $\lambda=\lambda_{0}$ se for semicontínua superior e inferiormente.

O seguinte lema nos fornece uma caracterização da semicontinuidade superior e inferior da família de conjuntos $\left\{J_{\lambda}\right\}_{\lambda \in \Lambda}$. 


\section{Lema 1.39.}

(i) A família $\left\{J_{\lambda}\right\}_{\lambda \in \Lambda}$ é semicontínua superiormente em $\lambda=\lambda_{0}$ se toda seqüência $\left\{u_{\lambda_{n}}\right\}_{n \in \mathbb{N}}$, com $u_{\lambda_{n}} \in J_{\lambda_{n}}, \lambda_{n} \rightarrow \lambda_{0}$, possui uma subseqüência convergente para um elemento de $J_{\lambda_{0}}$.

(ii) Se $J_{\lambda_{0}}$ é compacto e para todo $u \in J_{\lambda_{0}}$, existe uma seqüência $\left\{u_{\lambda_{n}}\right\}_{n \in \mathbb{N}} \operatorname{com} u_{\lambda_{n}} \in J_{\lambda_{n}}, \lambda_{n} \rightarrow \lambda_{0}$, tal que $u_{\lambda_{n}} \rightarrow u$, então a família $\left\{J_{\lambda}\right\}_{\lambda \in \Lambda}$ é semicontínua inferiormente em $\lambda=\lambda_{0}$.

\subsection{Grau Topológico}

Nesta seção desenvolveremos a teoria do Grau Topológico de Leray-Schauder. Este invariante, além de garantir a existência de solução para certas classes de problemas elípticos, garante também a existência de solução para perturbações destes problemas. Uma vez que esta teoria é não usual na maioria dos cursos de Análise Matemática, nos permitimos tomar uma pequena parte deste texto para construir as ferramentas necessárias. Para uma leitura mais aprofundada deste tema sugerimos as referências $[19,36,49]$.

\subsubsection{Grau Topológico em dimensão finita}

Definição 1.40. Dados $\Omega \subset \mathbb{R}^{n}$ aberto e limitado, $f \in C\left(\bar{\Omega}, \mathbb{R}^{n}\right)$ e $y \in \mathbb{R}^{n} \backslash f(\partial \Omega)$, ao número inteiro $d(f, \Omega, y)$ chamamos de grau da aplicação $f$ com relação a $\Omega$ e $y$, onde $d:\left\{(f, \Omega, y): \Omega \subset \mathbb{R}^{n}\right.$ aberto e limitado, $\left.f \in C\left(\bar{\Omega}, \mathbb{R}^{n}\right), y \in \mathbb{R}^{n} \backslash f(\partial \Omega)\right\} \rightarrow \mathbb{Z}$ é qualquer função satisfazendo

(d1) $d(i d, \Omega, y)=1$, para todo $y \in \Omega$;

(d2) $d(f, \Omega, y)=d\left(f, \Omega_{1}, y\right)+d\left(f, \Omega_{2}, y\right)$, para quaisquer subconjuntos abertos e disjuntos $\Omega_{1}, \Omega_{2}$ de $\Omega$ tais que $y \notin f\left(\bar{\Omega} \backslash\left(\Omega_{1} \cup \Omega_{2}\right)\right)$;

(d3) $d(h(t, \cdot), \Omega, y(t))$ é independente de $t \in[0,1]$, sempre que $h:[0,1] \times \bar{\Omega} \rightarrow \mathbb{R}^{n}$ é contínua, $y:$ $[0,1] \rightarrow \mathbb{R}^{n}$ é contínuo e $y(t) \notin h(t, \partial \Omega)$ para todo $t \in[0,1]$.

Teorema 1.41. Sejam $M=\left\{(f, \Omega, y) ; \Omega \subset \mathbb{R}^{n}\right.$ aberto e limitado $\left., f \in C\left(\bar{\Omega}, \mathbb{R}^{n}\right), y \in \mathbb{R}^{n} \backslash f(\partial \Omega)\right\}$. Então existe no máximo uma função $d: M \rightarrow \mathbb{Z}$ satisfazendo as propriedades $(\mathrm{d} 1)-(\mathrm{d} 3)$ da Definição 1.40. 
Definição 1.42. Nas condições do Teorema 1.41 a função d é chamada o Grau de Brower em $\mathbb{R}^{n}$.

Exemplo 1.43. Sejam $\Omega \subset \mathbb{R}^{n}$ aberto e limitado, $f \in C^{1}\left(\bar{\Omega}, \mathbb{R}^{n}\right)$ e $S_{f}$ o conjunto dos pontos críticos de $f$. A função

$$
d(f, \Omega, y)=\sum_{x \in f^{-1}\{y\}} \operatorname{sgn} J_{f}(x), \quad y \in \mathbb{R}^{n} \backslash f\left(\partial \Omega \cup S_{f}\right)
$$

onde $J_{f}(x)$ é o determinante Jacobiano da Matriz $D f(x)$ e $\sum_{\emptyset}:=0$, satisfaz as propriedades $(\mathrm{d} 1)$ - (d3) da Definição 1.40.

Na prova do Teorema 1.41, mostra-se que se $g \in C^{2}\left(\bar{\Omega}, \mathbb{R}^{n}\right)$ é tal que $\|g-f\|_{\infty}<\operatorname{dist}(y, f(\partial \Omega))$, e $y^{1}$ é um valor regular de $g$ tal que $\left\|y-y^{1}\right\|<\operatorname{dist}(y, f(\partial \Omega))$, então $d\left(g, \Omega, y^{1}\right)=d(g, \Omega, y)=$ $d(f, \Omega, y)$. Logo podemos estender (1.7) para a classe de todas as funções contínuas definidas em $\bar{\Omega}$, e pelo Teorema 1.41 segue consistente a seguinte definição alternativa:

Definição 1.44. Sejam $\Omega \subset \mathbb{R}^{n}$ aberto e limitado, $f \in C\left(\bar{\Omega}, \mathbb{R}^{n}\right)$ e $y \in \mathbb{R}^{n} \backslash f(\partial \Omega)$. Definimos $d(f, \Omega, y):=d(g, \Omega, y)$, onde $g \in C^{2}\left(\bar{\Omega}, \mathbb{R}^{n}\right)$ é qualquer aplicação tal que $\|g-f\|_{\infty}<\operatorname{dist}(y, f(\partial \Omega))$.

Proposição 1.45. Nas condições da definição anterior,

$(\mathrm{d} 4) d(f, \Omega, y) \neq 0$ implica $f^{-1}\{y\} \neq \emptyset$;

$(\mathrm{d} 5) d(f, \Omega, \cdot)$ é constante em cada componente conexa de $\mathbb{R}^{n} \backslash f(\partial \Omega)$;

(d6) $d(f, \Omega, y)=d\left(f, \Omega_{1}, y\right)$ para todo subconjunto aberto $\Omega_{1}$ de $\Omega$ tal que $y \notin f\left(\bar{\Omega} \backslash \Omega_{1}\right)$;

(d7) $d(f, \Omega, y)=d(g, \Omega, y)$, sempre que $f_{\mid \partial \Omega}=g_{\mid \partial \Omega}$.

Se $K$ é uma componente conexa de $\mathbb{R}^{n} \backslash f(\partial \Omega)$, denotamos por $d(f, \Omega, K):=d(f, \Omega, y)$, qualquer que seja $y \in K$.

Teorema 1.46. Sejam $\Omega \subset \mathbb{R}^{n}$ aberto e limitado, $f \in C\left(\bar{\Omega}, \mathbb{R}^{n}\right), g \in C\left(\mathbb{R}^{n}, \mathbb{R}^{n}\right)$ e $K_{i}$ as componentes conexas limitadas de $\mathbb{R}^{n} \backslash f(\partial \Omega)$. Se $y \notin g f(\partial \Omega)$, então

$$
d(g f, \Omega, y)=\sum_{i} d\left(f, \Omega, K_{i}\right) d\left(g, K_{i}, y\right),
$$

onde somente um número finito de parcelas são não-nulas. 


\subsubsection{Grau Topológico em dimensão infinita}

Como mencionado no início da seção, buscamos um invariante que garanta a existência de solução para certas classes de equações diferenciais parciais. Mas, em geral, nosso conceito de solução vive em espaços mais gerais que os espaços euclidianos $\mathbb{R}^{n}$, e uma extensão do nosso conceito de grau faz-se necessária. Entretanto para que isso seja possível nos vemos obrigados a restringir nossa classe de aplicações.

Definição 1.47. Sejam $X, Y$ espaços de Banach, $e \Omega \subset X$. Uma aplicação $F: \Omega \rightarrow Y$ é compacta se $F$ é contínua e $F(\Omega)$ é um subconjunto relativamente compacto de $X$. Denotaremos por $\mathscr{K}(\Omega, Y):=o$ conjunto das aplicações compactas de $\Omega$ em $Y$.

Teorema 1.48. Sejam $X$ um espaço de Banach real e $I: X \rightarrow X$ o operador identidade em $X$. Então existe uma única função

$$
D:\{(I-F, \Omega, y): \Omega \subset X \text { é aberto e limitado, } F \in \mathscr{K}(\bar{\Omega}, X), y \notin(I-F)(\partial \Omega)\} \rightarrow \mathbb{Z}
$$

satisfazendo

(D1) $D(I, \Omega, y)=1$, para todo $y \in \Omega$;

(D2) $D(I-F, \Omega, y)=\left(I-F, \Omega_{1}, y\right)+\left(I-F, \Omega_{2}, y\right)$ sempre que $\Omega_{1}, \Omega_{2}$ forem abertos disjuntos de $\Omega$ e $y \notin(I-F)\left(\bar{\Omega} \backslash\left(\Omega_{1} \cup \Omega_{2}\right)\right)$;

(D3) $D(I-H(t, \cdot), \Omega, y(t))$ é independente de $t \in[0,1]$ sempre que $H:[0,1] \times \bar{\Omega} \rightarrow X$ é compacta, $y:[0,1] \rightarrow X$ é contínua e $y(t) \notin(I-H(t, \cdot))(\partial \Omega)$ qualquer que seja $t \in[0,1]$.

Demonstração: Primeiramente mostremos que $D$ é unicamente determinada pelas propriedades (D1) - (D3).

Se $F \in \mathscr{K}(\bar{\Omega}, X)$, então $(I-F)(\partial \Omega) \subset X$ é fechado. Logo, se $y \notin(I-F)(\partial \Omega)$ então $\rho=$ $\operatorname{dist}(y,(I-F)(\partial \Omega))>0$, e existe $F_{1} \in \mathscr{K}(\bar{\Omega}, X)$ de posto finito tal que sup $\left\|F x-F_{1} x\right\|<\rho$. Por (D3), tomando $H(t, x)=F x+t\left(F_{1} x-F x\right)$ e $y(t) \equiv y$, vemos que $D(I-F, \Omega, y)=D\left(I-F_{1}, \Omega, y\right)$.

Seja $X_{1}$ um subespaço de $X$, com $\operatorname{dim} X_{1}<\infty$ tal que $y \in X_{1}, F_{1}(\bar{\Omega}) \subset X_{1}$ e $\Omega_{1}:=\Omega \cap X_{1} \neq \emptyset$. Existe uma projeção $P_{1}: X \rightarrow X_{1}$ contínua, de modo que podemos decompor $X=X_{1} \oplus X_{2}$ (com respeito a $\left.P_{1}\right)$. Observemos ainda que $X_{2}$ é fechado, uma vez que $\left(I-P_{1}\right)$ também é contínua. Seja $\tilde{F}_{1}: X_{1} \rightarrow X_{1}$ qualquer extensão contínua de $F_{\left.1\right|_{\bar{\Omega}_{1}}}$. Novamente por (D3), agora aplicado a $H(t, x)=t F_{1} x+(1-t) \tilde{F}_{1} P_{1} x$ e $y(t) \equiv y$, segue que $D\left(I-F_{1}, \Omega, y\right)=D\left(I-\tilde{F}_{1} P_{1}, \Omega, y\right)$. Mas todas as soluções em $\Omega$ de $x-\tilde{F}_{1} x=y$ e $x-\tilde{F}_{1} P_{1} x=y$ pertencem a $\Omega_{1}$, de modo que se $B_{1}(0)$ é a bola 
unitária de $X_{2}$, pelas propriedades (D3) e (D2) tem-se que $D(I-F, \Omega, y)=D\left(I-F_{1}, \Omega, y\right)=D\left(I-\tilde{F}_{1} P_{1}, \Omega, y\right)=D\left(I-\tilde{F}_{1} P_{1}, \Omega_{1}+B_{1}(0), y\right)=D\left(I-F_{1} P_{1}, \Omega_{1}+B_{1}(0), y\right)$. Agora, dados quaisquer $\Omega_{1} \subset X_{1}$ aberto e limitado , $f: \bar{\Omega}_{1} \rightarrow X_{1}$ contínua e $y \in X_{1} \backslash f\left(\partial \Omega_{1}\right)$, definimos

$$
d_{0}\left(f, \Omega_{1}, y\right):=D\left(f P_{1}+\left(I-P_{1}\right), \Omega_{1}+B_{1}(0), y\right) .
$$

Então as propriedades (D1) - (D3) implicam que $d_{0}$ satisfaz as propriedades $(\mathrm{d} 1)$ - (d3) da Definição 1.40 e portanto $d_{0}$ é o grau de Brower em $\mathbb{R}^{n} \cong X_{1}$. Em particular, tomando $f=$ $\left(I-F_{1}\right)_{\left.\right|_{\Omega \cap X_{1}}}$,

$$
D(I-F, \Omega, y)=D\left(I-F_{1}, \Omega, y\right)=D\left(\left(I-F_{1}\right)_{\left.\right|_{\Omega \cap X_{1}}}, \Omega, y\right) .
$$

Pode-se mostrar que $D$ não depende da particular escolha do subespaço $X_{1}$. A unicidade de $D$ decorre portanto da unicidade de $d_{0}$.

Proposição 1.49. Nas condições do Teorema 1.48 seguem as seguintes propriedades:

(D4) Se $D(I-T, \Omega, 0) \neq 0$, então $T$ possui ao menos um ponto fixo em $\Omega$;

(D5) $D(I-T, \tilde{\Omega}, 0)=D(I-T, \Omega, 0)$, para qualquer aberto $\Omega \subset \subset \Omega$ tal que $T$ não possua ponto fixo em $\bar{\Omega} \backslash \tilde{\Omega}$. Em particular, para um ponto fixo isolado $u^{*}$ de $T, D(I-T, \tilde{\Omega}, 0)$ não depende da vizinhança suficientemente pequena $\tilde{\Omega}$ de $u^{*}$ e é chamado o índice de $u^{*}$, e denotado por $\operatorname{ind}\left(u^{*}, T\right)$;

(D6) $D(I-T, \Omega, 0)=D\left(\left.(I-T)\right|_{\Omega_{0}}, \Omega_{0}, 0\right)$, se $T(\bar{\Omega}) \subseteq E$ é um subespaço fechado de $X$ e $\Omega_{0}:=\Omega \cap E$;

(D7) $D(I-T, \Omega, 0)=D\left(B(I-T) B^{-1}, B(\Omega), 0\right)$ para qualquer isomorfismo contínuo $B \in \mathscr{L}(X, Y)$;

(D8) Se $T$ é Frechet diferenciável no ponto fixo $u^{*}$ de $T$ e se $\operatorname{Ker}\left(I-T^{\prime}\left(u^{*}\right)\right)=\{0\}$, então o ponto fixo $u^{*}$ é isolado e $\left|\operatorname{ind}\left(u^{*}, T\right)\right|=1$.

Proposição 1.50. Sejam X um espaço de Banach real, $L_{0}, M_{0} \in \mathscr{K}(X)$. Se $L=I-L_{0}$ e $M=I-M_{0}$ são 1-1, então

$$
D(L M, \Omega, 0)=D(L, \Omega, 0) D(M, \Omega, 0),
$$

para todo aberto limitado $\Omega \subset X$ tal que $0 \in \Omega$. Além disso, se $X=\underset{1=1}{m} X_{i}$ se escreve como soma direta dos subespaços $X_{1}, \cdots, X_{m}$ com $L_{0}\left(X_{i}\right) \subset X_{i}$, então

$$
D\left(L, B_{1}(0), 0\right)=\prod_{i=1}^{m} D\left(\left.L\right|_{X_{i}}, B_{1}(0) \cap X_{i}, 0\right) .
$$


Teorema 1.51. Sejam $X$ um espaço de Banach real, $L \in \mathscr{K}(X)$ e $\lambda \neq 0$ tal que $\lambda^{-1}$ não é um autovalor de L. Seja também $\Omega \subset X$ aberto e limitado com $0 \in \Omega$. Então

$$
D(I-\lambda L, \Omega, 0)=(-1)^{m(\lambda)},
$$

onde $m(\lambda)$ é a soma das multiplicidades algébricas dos autovalores $\mu$ tais que $\mu \lambda>1$, e $m(\lambda)=0$ se $L$ não possui autovalores $\mu$ deste tipo.

Como ilustração do Teorema 1.51 consideremos o seguinte exemplo:

Exemplo 1.52. Consideremos o seguinte problema de valor de fronteira

$$
\left\{\begin{array}{c}
x^{\prime \prime}+\mu x=0 \\
x(0)=x(1)=0
\end{array}\right.
$$

que é equivalente ao problema de encontrar $x \in C([0,1])$ tal que

$$
x(t)-\mu \int_{0}^{1} k(t, s) x(s) d s=0, \quad t \in[0,1],
$$

onde

$$
k(t, s)= \begin{cases}s(1-t), & 0 \leqslant s \leqslant t \leqslant 1 \\ t(1-s), & 0 \leqslant t \leqslant s \leqslant 1\end{cases}
$$

Sejam $X=C([0,1])$ e $L: X \rightarrow X$ dado por $L x(t)=\int_{0}^{1} k(t, s) x(s) d s$. Como $k$ é contínua, $L$ é um operador linear compacto. Logo, uma solução não trivial $x \in X$ de (1.11) $(x-\mu L x=0)$ existe se, e somente se, $\mu^{-1}$ é um autovalor de L. Para $\mu \leqslant 0$ a solução geral de (1.10) é

$$
x(t)=\left\{\begin{array}{cc}
c e^{\sqrt{-\mu} t}+d e^{-\sqrt{-\mu} t}, & \mu<0 \\
c+d t, & \mu=0
\end{array}\right.
$$

e as condições de fronteira implicam que $d=c=0$.

Por outro lado, se $\mu>0$, temos

$$
x(t)=c \sin (\sqrt{\mu} t)+d \cos (\sqrt{\mu} t) .
$$

Novamente, as condições de fronteira implicam $d=0$ e $c \sin (\sqrt{\mu})=0$. Isto é possível para $c \neq 0$ se, e somente se, $\mu=n^{2} \pi^{2}$, para algum $n \in \mathbb{N}$, ou seja, os autovalores $\lambda_{n}$ de $L$ são da forma $\lambda_{n}=\left(n^{2} \pi^{2}\right)^{-1}$ para $n \geqslant 1$. Além disso, cada auto-espaço $N\left(\lambda_{n} I-L\right)$ é um subespaço unidimensional de $X$ gerado por $x_{n}(t)=\sin (n \pi t)$ e a multiplicidade algébrica de cada $\lambda_{n}$ é 1. Logo, do Teorema 1.51

$$
D\left(I-\lambda L, B_{1}(0), 0\right)=\left\{\begin{array}{rll}
1 & \text { para } & -\infty<\lambda<\pi^{2} \\
(-1)^{n} & \text { para } & n^{2} \pi^{2}<\lambda<(n+1)^{2} \pi^{2} .
\end{array}\right.
$$





\title{
Capítulo 2 Convergência compacta e continuidade dos atratores
}

\begin{abstract}
$\mathcal{U}$
ma das principais dificuldades no tratamento de problemas envolvendo perturbação de fronteira em equações diferenciais, é que as soluções geralmente vivem em espaços diferentes. Neste capítulo daremos um tratamento suficientemente adequado para nossa teoria de aproximação. Posteriormente veremos como o comportamento espectral dos operadores lineares $A_{\varepsilon}$, determinam o comportamento da dinâmica não linear do semigrupo $\left\{T_{\varepsilon}(t) ; t \geqslant 0\right\}$.
\end{abstract}




\subsection{Teoria linear}

Como já dissemos, seguimos interessados em estudar fenômenos que podem ser descritos formalmente como uma equação da forma,

$$
\dot{u}_{\varepsilon}+A_{\varepsilon} u_{\varepsilon}=F_{\varepsilon}\left(u_{\varepsilon}\right), \quad \varepsilon \in[0,1],
$$

em um espaço de Banach $X_{\varepsilon}$. Em geral o operador $A_{\varepsilon}$ é linear e atua em um subespaço próprio do espaço de fase $X_{\mathcal{\varepsilon}}$. Nossa técnica para estudar a continuidade do comportamento assintótico de (2.1) consiste num estudo cuidadoso do operador linear $A_{\varepsilon}$ obtido através das propriedades de convergência, em um sentido a ser descrito, das soluções dos problemas lineares

$$
A_{\varepsilon} u_{\varepsilon}=f_{\varepsilon} \in X_{\varepsilon} \quad \text { e } \quad A_{0} u_{0}=f_{0} \in X_{0} .
$$

Seja $\left\{X_{\varepsilon}\right\}_{\varepsilon \in[0,1]}$ uma família de espaços de Banach, e suponhamos que exista uma família de operadores $E_{\varepsilon} \in \mathscr{L}\left(X_{0}, X_{\varepsilon}\right)$ tais que

$$
\left\|E_{\varepsilon} u\right\|_{X_{\varepsilon}} \stackrel{\varepsilon \rightarrow 0}{\longrightarrow}\|u\|_{X_{0}}
$$

qualquer que seja $u \in X_{0}$. Como conseqüência do Príncípio da Limitação Uniforme (Generalizado) existem constantes $\tilde{\varepsilon} \in(0,1]$ e $M>0$ tais que $\sup _{[0, \tilde{\varepsilon}]}\left\|E_{\varepsilon}\right\|_{\mathscr{L}\left(X_{0}, X_{\varepsilon}\right)} \leqslant M$. De fato, seja $X_{n}=\left\{x \in X_{0}:\left\|E_{\varepsilon} x\right\|_{X_{\varepsilon}} \leqslant n, \forall \varepsilon \in[0,1]\right\} \subset X_{0}$. Observemos que $X_{n}$ é fechado e de (2.3) $X_{0}=\cup_{n=1}^{\infty} X_{n}$. Do Teorema de Baire existe $n_{0} \geqslant 1$ tal que int $X_{n_{0}} \neq \emptyset$. Sejam $x_{0} \in X_{0}$ e $r>0$ tais que $B\left(x_{0}, r\right) \subset X_{n_{0}}$. Assim $\left\|E_{\varepsilon}\left(x_{0}+r z\right)\right\|_{X_{\varepsilon}} \leqslant n_{0}$ quaisquer que sejam $\varepsilon \in[0,1]$ e $z \in B(0,1)$. Portanto

$$
r\left\|E_{\varepsilon} z\right\|_{X_{\varepsilon}} \leqslant\left\|E_{\varepsilon}\left(x_{0}+r z\right)\right\|_{X_{\varepsilon}}+\left\|E_{\varepsilon} x_{0}\right\|_{X_{\varepsilon}} \leqslant 2 n_{0} .
$$

A afirmação segue tomando o supremo no lado esquerdo para $\varepsilon \in[0,1]$ e $z \in B(0,1)$.

Definição 2.1. Dizemos que uma família $\left\{u_{\varepsilon}\right\}_{\varepsilon \in(0,1]}, u_{\varepsilon} \in X_{\mathcal{\varepsilon}}, E$-converge para $u_{0} \in X_{0}$ se $\left\|u_{\varepsilon}-E_{\varepsilon} u_{0}\right\|_{X_{\varepsilon}} \stackrel{\varepsilon \rightarrow 0}{\longrightarrow} 0$. Escrevemos $u_{\varepsilon} \stackrel{E}{\longrightarrow} u$.

Com esta noção de convergência introduzimos uma noção de compacidade.

Definição 2.2. Uma sequência $\left\{u_{\varepsilon_{n}}\right\}_{n \in \mathbb{N}}$, com $u_{\varepsilon_{n}} \in X_{\varepsilon_{n}}$ e $\varepsilon_{n} \rightarrow 0$, é E-relativamente compacta, se para toda subsequência $\left\{u_{\varepsilon_{n^{\prime}}}\right\}$ de $\left\{u_{\varepsilon_{n}}\right\}$ existem uma subsequência $\left\{u_{\varepsilon_{n^{\prime \prime}}}\right\}$ de $\left\{u_{\varepsilon_{n^{\prime}}}\right\}$ e $u \in X_{0}$ tais que $u_{\varepsilon_{n^{\prime \prime}}} \stackrel{E}{\longrightarrow} u$. A família $\left\{u_{\varepsilon}\right\}_{\varepsilon \in(0,1]}$ é E-relativamente compacta se toda sequência $\left\{u_{\varepsilon_{n}}\right\}$, com $\varepsilon_{n} \rightarrow 0$, é E-relativamente compacta. 
Definição 2.3. A família de operadores $\left\{B_{\varepsilon} \in \mathscr{L}\left(X_{\varepsilon}\right)\right\}_{\varepsilon \in(0,1]} E$-converge para o operador $B \in \mathscr{L}\left(X_{0}\right)$, se $B_{\varepsilon} u_{\varepsilon} \stackrel{E}{\longrightarrow}$ Bu sempre que $u_{\varepsilon} \stackrel{E}{\longrightarrow} u \in X_{0}$. Escrevemos $B_{\varepsilon} \stackrel{E E}{\longrightarrow} B$.

Definição 2.4. Uma família de operadores compactos $\left\{B_{\varepsilon} \in \mathscr{L}\left(X_{\varepsilon}\right)\right\}_{\varepsilon \in(0,1]}$ converge compactamente para um operador compacto $B \in \mathscr{L}\left(X_{0}\right)$ se para toda familia $\left\{u_{\varepsilon}\right\}_{\varepsilon \in(0,1]}$ com $\left\|u_{\varepsilon}\right\|_{X_{\varepsilon}}=1$, a familia $\left\{B_{\varepsilon} u_{\varepsilon}\right\}_{\varepsilon \in(0,1]}$ é E-relativamente compacta e $B_{\varepsilon} \stackrel{E E}{\longrightarrow} B$. Escrevemos $B_{\varepsilon} \stackrel{C C}{\longrightarrow} B$.

Lema 2.5. Se $B_{\varepsilon} \stackrel{C C}{\longrightarrow} B_{0}$ então existe $\varepsilon_{0}>0$ e uma constante $M>0$ tal que $\left\|B_{\varepsilon}\right\|_{\mathscr{L}\left(X_{\varepsilon}\right)} \leqslant M$, para todo $\varepsilon \in\left(0, \varepsilon_{0}\right]$.

Demonstração: Suponhamos que isso não seja verdade, ou seja, suponhamos que existam sequências $\varepsilon_{n} \stackrel{n \rightarrow \infty}{\longrightarrow} 0$ e $u_{\mathcal{E}_{n}} \in X_{\varepsilon_{n}}$ com $\left\|u_{\varepsilon_{n}}\right\|_{X_{\varepsilon_{n}}}=1$ tais que $\left\|B_{\varepsilon_{n}} u_{\mathcal{E}_{n}}\right\|_{X_{\varepsilon_{n}}} \stackrel{n \rightarrow \infty}{\longrightarrow}$. Mas isto contradiz a convergência compacta de $B_{\varepsilon}$ dada na Definição 2.4 .

Lema 2.6. Assuma que $B_{\varepsilon} \stackrel{C C}{\longrightarrow} B_{0}$. Suponha também que $\mathscr{N}\left(I+B_{0}\right)=\{0\}$. Então, existem $\varepsilon_{0}>0$ e $M>0$ tais que

$$
\left\|\left(I+B_{\varepsilon}\right)^{-1}\right\|_{\mathscr{L}\left(X_{\varepsilon}\right)} \leqslant M, \quad \forall \varepsilon \in\left(0, \varepsilon_{0}\right]
$$

Demonstração: Afirmamos que existe um $\tilde{\varepsilon_{0}}$ tal que $\mathscr{N}\left(I+B_{\varepsilon}\right)=\{0\}$, para todo $\varepsilon \in\left(0, \widetilde{\varepsilon_{0}}\right]$. De fato, suponha por absurdo, que existam sequências $\varepsilon_{n} \rightarrow 0$ e $u_{\varepsilon_{n}} \in \mathscr{N}\left(I+B_{\varepsilon_{n}}\right)$, com $\left\|u_{\varepsilon_{n}}\right\|_{X_{\varepsilon_{n}}}=1$. Como $\left\{B_{\varepsilon_{n}} u_{\varepsilon_{n}}\right\}$ é relativamente compacta, existem, uma subseqüência, (denotada também por $\left.\left\{B_{\varepsilon_{n}} u_{\varepsilon_{n}}\right\}\right)$, e $u_{0} \in X_{0}$, tais que $B_{\varepsilon_{n}} u_{\varepsilon_{n}} \stackrel{E}{\longrightarrow} u_{0}$ quando $n \rightarrow \infty$. Além disso, $u_{\varepsilon_{n}}+B_{\varepsilon_{n}} u_{\varepsilon_{n}}=0$ o que implica $u_{\varepsilon_{n}} \stackrel{E}{\longrightarrow}-u$, e portanto $\left(I+B_{0}\right) u_{0}=0$, com $\left\|u_{0}\right\|_{X_{0}}=1$, o que contradiz a hipótese.

Agora, da compacidade dos operadores $B_{\varepsilon}$, segue que os operadores $\left(I+B_{\varepsilon}\right)^{-1}$ estão bem definidos e são limitados para cada $\varepsilon \in\left(0, \tilde{\varepsilon}_{0}\right]$.

Observemos que mostrar (2.4) é equivalente a mostrar

$$
\left\|\left(I+B_{\varepsilon}\right) u_{\varepsilon}\right\|_{X_{\varepsilon}} \geqslant \frac{1}{M}, \quad \forall \varepsilon \in\left(0, \varepsilon_{0}\right] \text { e } \forall u_{\varepsilon} \in X_{\varepsilon} \text { com }\left\|u_{\varepsilon}\right\|_{X_{\varepsilon}}=1 .
$$

Suponha que isto não seja verdade, isto é, suponha que exista uma sequência $\left\{u_{\varepsilon_{n}}\right\}$, com $u_{\varepsilon_{n}} \in X_{\varepsilon_{n}}$, $\left\|u_{\varepsilon_{n}}\right\|_{X_{\varepsilon_{n}}}=1$ e $\varepsilon_{n} \rightarrow 0$ tal que $\left\|\left(I+B_{\varepsilon_{n}}\right) u_{\varepsilon_{n}}\right\|_{X_{\varepsilon_{n}}} \rightarrow 0$. Desde que $u_{\varepsilon_{n}}+B_{\varepsilon_{n}} u_{\varepsilon_{n}} \rightarrow 0$, e $\left\{B_{\varepsilon_{n}} u_{\varepsilon_{n}}\right\}$ possui subsequência convergente (a qual continuaremos denotando por $\left\{B_{\varepsilon_{n}} u_{\varepsilon_{n}}\right\}$ ), que converge para $u_{0}$, com $\left\|u_{0}\right\|_{X_{0}}=1$, temos que $u_{\varepsilon_{n}} \stackrel{E}{\longrightarrow}-u_{0}$, o que implica novamente que $\left(I+B_{0}\right) u_{0}=0$ contradizendo nossa hipótese.

Como nas aplicações em geral os operadores $B_{\varepsilon}$ são inversas de operadores diferenciais $A_{\varepsilon}: D\left(A_{\varepsilon}\right) \subset X_{\varepsilon} \rightarrow X_{\varepsilon}$ adicionaremos as seguintes hipóteses: 
(CR) Os operadores $A_{\varepsilon}, \varepsilon \in[0,1]$ são fechados, densamente definidos com resolvente compacto, $0 \in \rho\left(A_{\varepsilon}\right)$ e ainda

$$
A_{\mathcal{\varepsilon}}^{-1} \stackrel{C C}{\longrightarrow} A_{0}^{-1}
$$

Lema 2.7. Para todo $\lambda \in \rho\left(A_{0}\right)$, existe um $\varepsilon_{\lambda}>0$ tal que $\lambda \in \rho\left(A_{\varepsilon}\right)$ para todo $\varepsilon \in\left[0, \varepsilon_{\lambda}\right]$, e existe uma constante $M_{\lambda}>0$ tal que

$$
\sup _{\varepsilon \in\left(0, \varepsilon_{\lambda}\right]}\left\|\left(\lambda-A_{\varepsilon}\right)^{-1}\right\|_{\mathscr{L}\left(X_{\varepsilon}\right)} \leqslant M_{\lambda}
$$

Além disso, $\left(\lambda-A_{\varepsilon}\right)^{-1} \stackrel{C C}{\longrightarrow}\left(\lambda-A_{0}\right)^{-1}$.

Demonstração: Seja $\lambda \in \rho\left(A_{0}\right)$. Então a igualdade $\lambda-A_{0}=-\left(I-\lambda A_{0}^{-1}\right) A_{0}$, implica a existência de $\left(I-\lambda A_{0}^{-1}\right)^{-1}$. Pelo Lema 2.6 existe $\varepsilon_{\lambda} \in\left(0, \varepsilon_{0}\right]$ tal que $\left(I-\lambda A_{\varepsilon}^{-1}\right)^{-1}$ está bem definido e é uniformemente limitado para todo $\varepsilon \in\left(0, \varepsilon_{\lambda}\right]$. Logo podemos definir o operador $\left(\lambda-A_{\varepsilon}\right)^{-1}=$ $-A_{\varepsilon}^{-1}\left(I-\lambda A_{\varepsilon}^{-1}\right)^{-1}$ e concluir que $\left\|\left(\lambda-A_{\varepsilon}\right)^{-1}\right\|_{\mathscr{L}\left(X_{\varepsilon}\right)} \leqslant M_{\lambda}, \quad \forall \varepsilon \in\left[0, \varepsilon_{\lambda}\right]$.

Agora, da limitação uniforme de $\left(I-\lambda A_{\varepsilon}^{-1}\right)^{-1}$ e da convergência compacta de $A_{\varepsilon}^{-1}$, dada uma sequência $\varepsilon_{n} \rightarrow 0$, com $u_{\varepsilon_{n}} \in X_{\varepsilon_{n}}$ e $\left\|u_{\varepsilon_{n}}\right\|_{\sum_{\varepsilon_{n}}}=1$, segue que $\left(\lambda-A_{\varepsilon_{n}}\right)^{-1} u_{\varepsilon_{n}}=-A_{\varepsilon_{n}}^{-1}\left(I-\lambda A_{\varepsilon_{n}}^{-1}\right)^{-1} u_{\varepsilon_{n}}$ possui subsequência $E$-convergente. Portanto, se $u_{\varepsilon} \stackrel{E}{\longrightarrow} u_{0}$, para cada sequência de $\left\{\left(\lambda-A_{\varepsilon}\right)^{-1} u_{\varepsilon}\right\}$ existem uma subsequência (também denotada por $\left\{\left(\lambda-A_{\varepsilon}\right)^{-1} u_{\varepsilon}\right\}$ ) e $v_{0} \in X_{0}$ tais que

$$
\left(\lambda-A_{\varepsilon}\right)^{-1} u_{\varepsilon}=-A_{\varepsilon}^{-1}\left(I-\lambda A_{\varepsilon}^{-1}\right)^{-1} u_{\varepsilon}=z_{\varepsilon} \stackrel{E}{\longrightarrow} v_{0} .
$$

Dessa forma,

$$
A^{-1} u \stackrel{E}{\longleftarrow} A_{\varepsilon}^{-1} u_{\varepsilon}=-\left(I-\lambda A_{\varepsilon}^{-1}\right) z_{\varepsilon} \stackrel{E}{\longrightarrow}-\left(I-\lambda A^{-1}\right) v_{0}
$$

e portanto $v_{0}=-A_{0}^{-1}\left(I-\lambda A_{0}^{-1}\right)^{-1} u_{0}=\left(\lambda-A_{0}\right)^{-1} u_{0}$, mostrando que $\left(\lambda-A_{\mathcal{E}}\right)^{-1} \stackrel{E E}{\longrightarrow}\left(\lambda-A_{0}\right)^{-1}$, ou seja, $\left(\lambda-A_{\varepsilon}\right)^{-1} \stackrel{C C}{\longrightarrow}\left(\lambda-A_{0}\right)^{-1}$.

Lema 2.8. Dados $\lambda \in \mathbb{C}$ e $\delta \in \mathbb{R}^{+}$, defina $S_{\lambda, \delta}=\{\mu \in \mathbb{C}:|\mu-\lambda|=\delta\}$. Se para algum $\delta>0$, $\sigma\left(A_{0}\right) \cap S_{\lambda, \delta}=\emptyset$, então existe $\varepsilon_{\delta}>0$ tal que $\sigma\left(A_{\varepsilon}\right) \cap S_{\lambda, \delta}=\emptyset$ para todo $\varepsilon \in\left(0, \varepsilon_{\delta}\right]$.

Demonstração: Suponha que não. Então existem sequências $\varepsilon_{n} \rightarrow 0, \mu_{n} \in S_{\lambda, \delta}$ (a qual podemos assumir convergente para $\left.\mu \in S_{\lambda, \delta}\right)$ e $u_{\varepsilon_{n}} \in X_{\varepsilon_{n}}^{0},\left\|u_{\varepsilon_{n}}\right\|_{\chi_{\varepsilon_{n}}}=1$, tais que $u_{\varepsilon_{n}}-\mu_{n} A_{\varepsilon_{n}}^{-1} u_{\varepsilon_{n}}=0$. As convergências $A_{\mathcal{\varepsilon}}^{-1} \stackrel{C C}{\longrightarrow} A_{0}^{-1}$ e $\mu_{n} \rightarrow \mu$ implicam na convergência $\mu_{n} A_{\mathcal{\varepsilon}_{n}}^{-1} \stackrel{C C}{\longrightarrow} \mu A_{0}^{-1}$ que por sua vez implica que a sequência $\left\{u_{\varepsilon_{n}}\right\},\left(u_{\varepsilon_{n}}=\mu_{n} A_{\varepsilon_{n}}^{-1} u_{\varepsilon_{n}}\right)$ possui subsequência convergente para $u_{0} \in X_{0}$, e $\|u\|_{X_{0}}=1$. Como $u_{\varepsilon_{n}}-\mu_{n} A_{\mathcal{\varepsilon}_{n}}^{-1} u_{\varepsilon_{n}}=0$ obtemos que $\mu-\mu A_{0}^{-1} u_{0}=0$, ou seja, $u_{0} \in \sigma\left(A_{0}\right)$. 
Recordemos que os operadores $A_{\varepsilon}$ possuem resolvente compacto, logo seus espectros $\sigma\left(A_{\varepsilon}\right)$, consistem apenas de autovalores isolados. No próximo resultado mostraremos que o espectro $\sigma\left(A_{\varepsilon}\right)$ de $A_{\varepsilon}$ aproxima-se do espectro $\sigma\left(A_{0}\right)$ de $A_{0}$ a medida que $\varepsilon \rightarrow 0$.

Teorema 2.9. As seguintes afirmações são válidas:

(i) Se para alguma sequência $\varepsilon_{n} \rightarrow 0$, temos que $\lambda_{n} \rightarrow \lambda_{0}$, com $\lambda_{n} \in \sigma\left(A_{\varepsilon_{n}}\right)$, então $\lambda_{0} \in \sigma\left(A_{0}\right)$.

(ii) Reciprocamente para todo $\lambda_{0} \in \sigma\left(A_{0}\right)$, existem sequências $\varepsilon_{n} \rightarrow 0$ e $\lambda_{n} \in \sigma\left(A_{\varepsilon_{n}}\right)$, tais que $\lambda_{n} \rightarrow \lambda_{0}$.

Demonstração: Consideremos sequências $\varepsilon_{n} \rightarrow 0$ e $\lambda_{n} \in \sigma\left(A_{\mathcal{\varepsilon}_{n}}\right)$, tais que $\lambda_{n} \rightarrow \lambda$. Sejam $u_{n} \in X_{\varepsilon_{n}}$, com $\left\|u_{n}\right\|_{\varepsilon_{\varepsilon_{n}}}=1$ tais que $\left(I-\lambda_{n} A_{\varepsilon_{n}}^{-1}\right) u_{n}=0$. Então

$$
\left\|\left(I-\lambda A_{\varepsilon_{n}}^{-1}\right) u_{n}\right\|_{X_{\varepsilon_{n}}} \leqslant\left\|\left(I-\lambda_{n} A_{\varepsilon_{n}}^{-1}\right) u_{n}\right\|_{X_{\varepsilon_{n}}}+\left\|\left(\lambda_{n}-\lambda\right) A_{\varepsilon_{n}}^{-1} u_{n}\right\|_{X_{\varepsilon_{n}}} \rightarrow 0 .
$$

Da convergência $\lambda A_{\varepsilon_{n}}^{-1} \stackrel{C C}{\longrightarrow} \lambda A_{0}^{-1}$, existe uma subsequência de $\left\{\lambda A_{\varepsilon_{n}}^{-1} u_{n}\right\}$ convergente a $v$. Como $\left(I-\lambda A_{\mathcal{\varepsilon}_{n}}^{-1}\right) u_{n} \rightarrow 0$ obtemos $u_{n} \stackrel{E}{\longrightarrow} v$ e $\|v\|_{X_{0}}=1$. Portanto $\left(I-\lambda A_{0}^{-1}\right) v=0$, mostrando que $\lambda \in$ $\rho\left(A_{0}\right)$.

Para a afirmação (ii), seja $\lambda \in \sigma\left(A_{0}\right)$ e considere $O_{\lambda, \delta}=\{\mu \in \mathbb{C}:|\mu-\lambda| \leqslant \delta\}$, com $\delta$ tal que $O_{\lambda, \delta} \cap \sigma\left(A_{0}\right)=\{\lambda\}$. Mostraremos que existe $\bar{\varepsilon} \in(0,1]$ tal que $O_{\lambda, \delta} \cap \sigma\left(A_{\varepsilon}\right) \neq \varnothing$, para todo $\varepsilon \in(0, \bar{\varepsilon}]$.

Primeiramente afirmamos que existe $\varepsilon_{1} \in(0,1]$ tal que os operadores $\left(\mu-A_{\varepsilon}\right)^{-1}$ estão bem definidos e são uniformemente limitados para todo $\varepsilon_{1} \in(0,1]$ e todo $\mu \in \partial O_{\lambda, \delta}=S_{\lambda, \delta}$.

Para a primeira parte, o Lema 2.8 garante a existência de $\varepsilon_{\delta}$ tal que $S_{\lambda, \delta} \subset \rho\left(A_{\varepsilon}\right)$ para todo $\varepsilon \in\left(0, \varepsilon_{\delta}\right]$. Agora, mostrar que $\left(\mu-A_{\varepsilon}\right)^{-1}$ é uniformemente limitado para todo $\varepsilon \in\left(0, \varepsilon_{1}\right]$ e todo $\mu \in S_{\lambda, \delta}$, uma vez que $\left\{\left\|A_{\varepsilon}^{-1}\right\|_{\mathscr{L}\left(X_{\varepsilon}\right)^{0}}, \varepsilon \in\left(0, \varepsilon_{0}\right]\right\}$ é limitado, é suficiente mostrar que os operadores $\left(I-\mu A_{\varepsilon}^{-1}\right)^{-1}$ são uniformemente limitados para todo $\varepsilon \in\left(0, \varepsilon_{1}\right]$ e todo $\mu \in S_{\lambda, \delta}$.

Suponha que esta última não se verifique, isto é, suponha que existam sequências $\varepsilon_{n} \rightarrow 0$, $\left\{\mu_{n}\right\} \subset S_{\lambda, \delta}$ (que podemos assumir convergente a $\mu$ ), e $u_{n} \in X_{\varepsilon_{n}},\left\|u_{n}\right\|_{X_{\varepsilon_{n}}}=1$, tais que

$$
\left\|\left(I-\mu_{n} A_{\varepsilon_{n}}^{-1}\right)^{-1} u_{n}\right\|_{X_{\varepsilon_{n}}} \rightarrow \infty \text {, ou equivalentemente, }\left\|\left(I-\mu_{n} A_{\varepsilon_{n}}^{-1}\right) u_{n}\right\|_{X_{\varepsilon_{n}}} \rightarrow 0 .
$$

Agora, da convergência $A_{\varepsilon}^{-1} \stackrel{C C}{\longrightarrow} A_{0}^{-1}$, (tomando subsequência se necessário) $\mu_{n} A_{\varepsilon}^{-1} u_{n}=\left(\mu_{n}-\right.$ $\mu) A_{\varepsilon}^{-1} u_{n}+\mu A_{\varepsilon}^{-1} u_{n} \stackrel{E}{\longrightarrow} v$. E como $\left(I-\mu_{n} A_{\varepsilon_{n}}^{-1}\right) u_{n} \rightarrow 0$, implica $u_{n} \stackrel{E}{\longrightarrow} v$ e $\|v\|_{X_{0}}=1$. Logo $(I-$ $\left.\mu A_{0}^{-1}\right) v=0$, absurdo pois, $\mu \in \rho\left(A_{0}\right)$. Tome $\bar{\varepsilon}=\min \left\{\varepsilon_{\delta}, \varepsilon_{1}\right\}$.

Agora vamos supor que $O_{\lambda, \delta} \subset \rho\left(A_{\varepsilon}\right)$ para todo $\varepsilon \in(0, \bar{\varepsilon}]$. Sendo a função $O_{\lambda, \delta} \ni \mu \mapsto(I-$ $\left.\mu A_{0}^{-1}\right)^{-1}$ holomorfa, e limitada em $S_{\lambda, \delta}$, do Teorema do Máximo Módulo existe uma constante 
$C>0$, tal que

$$
\left\|\left(I-\lambda A_{\varepsilon}^{-1}\right)^{-1}\right\|_{\mathscr{L}\left(X_{\varepsilon}\right)} \leqslant \max _{\substack{\mu \in S_{\lambda, \delta} \\ \varepsilon \in(0, \bar{\varepsilon}]}}\left\|\left(I-\mu A_{\varepsilon}^{-1}\right)^{-1}\right\|_{\mathscr{L}\left(X_{\varepsilon}\right)} \leqslant C .
$$

Logo, se $\varepsilon_{n} \rightarrow 0$ e $X_{\varepsilon_{n}} \ni u_{n} \stackrel{E}{\longrightarrow} u \in X_{0}$,

$$
\left\|\left(I-\lambda A_{0}^{-1}\right) u\right\|_{X_{0}}=\lim _{n \rightarrow \infty}\left\|\left(I-\lambda A_{\varepsilon_{n}}^{-1}\right) u_{n}\right\|_{X_{\varepsilon_{n}}} \geqslant \frac{1}{C}\|u\|_{X_{0}}
$$

mostrando que $\lambda \in \rho\left(A_{0}\right)$. Portanto $O_{\lambda, \delta}$ intercepta $\sigma\left(A_{\varepsilon}\right)$, para $\varepsilon$ suficientemente pequeno.

O próximo resultado generaliza o Lema 2.8.

Lema 2.10. Se $K$ é um subconjunto compacto de $\rho\left(A_{0}\right)$, então existe uma constante $\varepsilon_{K}>0$ tal que $K \subset \rho\left(A_{\varepsilon}\right)$ para todo $\varepsilon \in\left(0, \varepsilon_{K}\right] e$

$$
\sup _{\substack{\mu \in K \\ \varepsilon \in\left(0, \varepsilon_{K}\right]}}\left\|\left(\mu-A_{\varepsilon}\right)^{-1}\right\|_{\mathscr{L}\left(X_{\varepsilon}\right)}<\infty .
$$

Além disso, para todo $u \in X_{0}$

$$
\sup _{\mu \in K}\left\|\left(\mu-A_{\varepsilon}\right)^{-1} E_{\varepsilon} u-E_{\varepsilon}\left(\mu-A_{0}\right)^{-1} u\right\|_{X_{\varepsilon}} \stackrel{\varepsilon \rightarrow 0}{\longrightarrow} 0 .
$$

Demonstração: Vamos primeiramente provar que existe $\varepsilon_{K}>0$ tal que $K \subset \rho\left(A_{\varepsilon}\right)$ para todo $\varepsilon \in\left(0, \varepsilon_{K}\right]$. Suponhamos que existam sequências $\varepsilon_{n} \rightarrow 0$ e $\left\{\mu_{n}\right\} \subset K$ (podemos assumir $\mu_{n} \rightarrow \mu \in K$ ) onde cada $\mu_{n}$ é um autovalor de $A_{\varepsilon_{n}}$. Do item (i), do Teorema $2.9 \mu \in \sigma(A)$, o que é uma contradição.

Para provar (2.6), é suficiente mostrar que $\sup _{\substack{\mu \in K \\ \varepsilon \in\left(0, \varepsilon_{k}\right]}}\left\|\left(I-\mu A_{\varepsilon}^{-1}\right)^{-1}\right\|_{\mathscr{L}\left(X_{\varepsilon}\right)}<\infty$.

Novamente procedemos por redução ao absurdo. Assuma que existam sequências $\varepsilon_{n} \rightarrow 0$, $\left\{\mu_{n}\right\} \subset K$, com $\mu_{n} \rightarrow \mu \in K$ tal que $\left\|\left(I-\mu_{n} A_{\varepsilon_{n}}^{-1}\right)^{-1}\right\|_{\mathscr{L}\left(X_{\varepsilon_{n}}\right)} \rightarrow \infty$. Como $\mu_{n} A_{\varepsilon_{n}}^{-1}$ converge compactamente para $\mu A^{-1}$, isto contradiz o Lema 2.6.

Seja $u \in U$. Para provar (2.7), suponha por absurdo que existam sequências $\varepsilon_{n} \rightarrow 0, K \ni \mu_{n} \rightarrow$ $\mu \in K$ e $\eta>0$ tais que

$$
\left\|\left(\mu_{n}-A_{\varepsilon_{n}}\right)^{-1} E_{\varepsilon_{n}} u-E_{\varepsilon_{n}}\left(\mu_{n}-A_{0}\right)^{-1} u\right\|_{X_{\varepsilon_{n}}} \geqslant \eta
$$

Da identidade do resolvente,

$$
\left(\mu_{n}-A_{\varepsilon_{n}}\right)^{-1} E_{\varepsilon_{n}} u-\left(\mu-A_{\varepsilon_{n}}\right)^{-1} E_{\varepsilon_{n}} u=\left(\mu-\mu_{n}\right)\left(\mu_{n}-A_{\varepsilon_{n}}\right)^{-1}\left(\mu-A_{\varepsilon_{n}}\right)^{-1} E_{\varepsilon_{n}} u,
$$

e de $(2.6)$

$$
\left\|\left(\mu_{n}-A_{\varepsilon_{n}}\right)^{-1} E_{\varepsilon_{n}} u-\left(\mu-A_{\varepsilon_{n}}\right)^{-1} E_{\varepsilon_{n}} u\right\|_{X_{\varepsilon_{n}}} \rightarrow 0 .
$$


Do Lema 2.7 como $E_{\mathcal{\varepsilon}_{n}} u \stackrel{E}{\longrightarrow} u$

$$
\left\|\left(\mu-A_{\varepsilon_{n}}\right)^{-1} E_{\varepsilon_{n}} u-E_{\varepsilon_{n}}\left(\mu-A_{0}\right)^{-1} u\right\|_{X_{\varepsilon_{n}}} \rightarrow 0,
$$

Agora, da continuidade do resolvente,

$$
\left\|\left(\mu_{n}-A_{0}\right)^{-1} u-\left(\mu-A_{0}\right)^{-1} u\right\|_{X_{0}} \rightarrow 0 \Rightarrow\left\|E_{\varepsilon_{n}}\left(\mu_{n}-A_{0}\right)^{-1} u-E_{\varepsilon_{n}}\left(\mu-A_{0}\right)^{-1} u\right\|_{X_{\varepsilon_{n}}} \rightarrow 0,
$$

e deste modo,

$$
\begin{aligned}
\eta \leqslant\left\|\left(\mu_{n}-A_{\varepsilon_{n}}\right)^{-1} E_{\varepsilon_{n}} u-E_{\varepsilon_{n}}\left(\mu_{n}-A_{0}\right)^{-1} u\right\|_{X_{\varepsilon_{n}}} & \leqslant\left\|\left(\mu_{n}-A_{\varepsilon_{n}}\right)^{-1} E_{\varepsilon_{n}} u-\left(\mu-A_{\varepsilon_{n}}\right)^{-1} E_{\varepsilon_{n}} u\right\|_{X_{\varepsilon_{n}}} \\
& +\left\|\left(\mu-A_{\varepsilon_{n}}\right)^{-1} E_{\varepsilon_{n}} u-E_{\varepsilon_{n}}\left(\mu-A_{0}\right)^{-1} u\right\|_{X_{\varepsilon_{n}}} \\
& +\left\|E_{\varepsilon_{n}}\left(\mu-A_{0}\right)^{-1} u-E_{\varepsilon_{n}}\left(\mu_{n}-A_{0}\right)^{-1} u\right\|_{X_{\varepsilon_{n}}} \rightarrow 0 .
\end{aligned}
$$

Definição 2.11. Para um ponto isolado $\lambda \in \sigma\left(A_{0}\right)$ associamos seu auto espaço generalizado $W\left(\lambda, A_{0}\right)=Q\left(\lambda, A_{0}\right) X_{0}$ onde

$$
Q\left(\lambda, A_{0}\right)=\frac{1}{2 \pi i} \int_{S_{\lambda, \delta}}\left(\xi I-A_{0}\right)^{-1} d \xi
$$

com $\delta$ suficientemente pequeno de modo a $\sigma\left(A_{0}\right) \backslash\{\lambda\}$ não interceptar o disco $O_{\lambda, \delta}$.

O Lema 2.8 garante a existência de $\varepsilon_{\delta}$ tal que $\rho\left(A_{\varepsilon}\right) \supset S_{\lambda, \delta}$ para todo $\varepsilon \leqslant \varepsilon_{\delta}$. Ficam então bem definidos os auto-espaços generalizados $W\left(\lambda, A_{\varepsilon}\right)=Q\left(\lambda, A_{\varepsilon}\right) X_{\mathcal{\varepsilon}}$ onde

$$
Q\left(\lambda, A_{\varepsilon}\right)=\frac{1}{2 \pi i} \int_{S_{\lambda, \delta}}\left(\xi I-A_{\varepsilon}\right)^{-1} d \xi
$$

Acerca das projeções $Q_{\varepsilon}(\lambda)=Q\left(\lambda, A_{\varepsilon}\right)$ e $Q(\lambda)=Q\left(\lambda, A_{0}\right)$, temos o seguinte resultado:

Lema 2.12. Sejam $\lambda \in \sigma\left(A_{0}\right)$ e $\delta$ suficientemente pequeno de modo a $\sigma\left(A_{0}\right) \backslash\{\lambda\}$ não interceptar o disco $O_{\lambda, \delta}$. Então $Q_{\varepsilon}(\lambda) \stackrel{C C}{\longrightarrow} Q(\lambda)$ quando $\varepsilon \rightarrow 0$.

Demonstração: A convergência $\left(\xi-A_{\varepsilon}\right)^{-1} \stackrel{C C}{\longrightarrow}\left(\xi-A_{0}\right)^{-1}$, para todo $\xi \in S_{\lambda, \delta}$, e a limitação uniforme de $\left\{\left\|\left(\xi+A_{\varepsilon}\right)^{-1}\right\|_{\mathscr{L}\left(X_{\varepsilon}\right)}\right\}$ onde $\varepsilon \in\left(0, \varepsilon_{0}\right]$ asseguram que pelo Teorema da Convergência Dominada de Lebesgue

$$
Q_{\varepsilon}(\lambda)=\frac{1}{2 \pi i} \int_{S_{\lambda, \delta}}\left(\xi-A_{\varepsilon}\right)^{-1} d \xi \stackrel{E E}{\longrightarrow} \frac{1}{2 \pi i} \int_{S_{\lambda, \delta}}\left(\xi-A_{0}\right)^{-1} d \xi=Q(\lambda) .
$$

Agora, se $u_{n} \in X_{\varepsilon_{n}}$ com $\left\|u_{n}\right\|_{X_{\varepsilon_{n}}}=1$, escrevendo $Q_{\varepsilon}(\lambda)=A_{\varepsilon}^{-1} \frac{1}{2 \pi i} \int_{S_{\lambda, \delta}}\left(I-\xi A_{\varepsilon}^{-1}\right)^{-1} d \xi$, e, pelo Lema $2.10,\left(I-\xi A_{\varepsilon}^{-1}\right)^{-1}$ é uniformemente limitado, $\frac{1}{2 \pi i} \int_{S_{\lambda, \delta}}\left(I-\xi A_{\varepsilon}^{-1}\right)^{-1} d \xi$ é limitada. Agora da 
convergência $A_{\varepsilon}^{-1} \stackrel{C C}{\longrightarrow} A_{0}^{-1}$, resulta que a família $\left\{Q_{\varepsilon_{n}}(\lambda) u_{n}\right\}$ possui subsequência convergente, e portanto, $Q_{\varepsilon}(\lambda) \stackrel{C C}{\longrightarrow} Q(\lambda)$ como queríamos.

Teorema 2.13. Com a notação introduzida acima, são válidas as seguintes afirmações

(i) Existe $\bar{\varepsilon}>0$ tal que $\operatorname{dim} W\left(\lambda, A_{\varepsilon}\right)=\operatorname{dim} W\left(\lambda, A_{0}\right)$ para todo $\varepsilon \in(0, \bar{\varepsilon}]$;

(ii) Para todo $u \in W\left(\lambda, A_{0}\right)$, existe uma sequência $u_{\varepsilon} \in W\left(\lambda, A_{\varepsilon}\right)$, tal que $u_{\varepsilon} \stackrel{E}{\longrightarrow} u$;

(iii) Toda sequência $\varepsilon_{n} \rightarrow 0, u_{n} \in W\left(\lambda, A_{\varepsilon_{n}}\right)$, com $\left\|u_{n}\right\|_{X_{\varepsilon_{n}}}=1$ possui subsequência $E$-convergente e todo ponto limite desta sequência pertence a $W\left(\lambda, A_{0}\right)$.

\section{Demonstração:}

(i) Mostremos inicialmente que que existe $\bar{\varepsilon}_{0}$ tal que posto $\left(Q_{\varepsilon}(\lambda)\right) \geqslant \operatorname{posto}(Q(\lambda))$ para todo $\varepsilon \in$ $\left(0, \bar{\varepsilon}_{0}\right]$. De fato, assuma que exista uma sequência $\varepsilon_{n} \rightarrow 0$ tal que posto $\left(Q_{\varepsilon}(\lambda)\right)<\operatorname{posto}(Q(\lambda))$. Se $\left\{v_{1}, \cdots v_{k}\right\}$ é uma base de $W\left(\lambda, A_{0}\right)=Q(\lambda) X_{0}$ então, $\left\{Q_{\varepsilon_{n}}(\lambda) E_{\varepsilon_{n}} v_{1}, \cdots Q_{\varepsilon_{n}}(\lambda) E_{\varepsilon_{n}} v_{k}\right\}$ é um conjunto linearmente dependente de $X_{\varepsilon_{n}}$. Escolha $\alpha_{1}^{n}, \cdots, \alpha_{k}^{n}$ tais que $\sum_{i=1}^{k}\left|\alpha_{i}^{n}\right|=1$ e $\sum_{i=1}^{k} \alpha_{i}^{n} Q_{\varepsilon_{n}}\left(\lambda_{0}\right) E_{\varepsilon_{n}} v_{i}=0$.

Tomando subsequências se necessário, podemos supor que existem $\alpha_{1}, \cdots, \alpha_{k}$ tais que $\alpha_{i}^{n} \rightarrow \alpha_{i}$, $1 \leqslant i \leqslant k . \operatorname{Logo}$

$$
\sum_{i=1}^{k} \alpha_{i} v_{i}=\sum_{i=1}^{k} \alpha_{i} Q\left(\lambda_{0}\right) v_{i}=\lim _{n \rightarrow \infty} \sum_{i=1}^{k} \alpha_{i}^{n} Q_{\varepsilon_{n}}\left(\lambda_{0}\right) E_{\varepsilon_{n}} v_{i}=0 \text { com } \sum_{i=1}^{k}\left|\alpha_{i}\right|=1
$$

contradizendo a independendência linear de $\left\{v_{1}, \cdots, v_{k}\right\}$. Isto implica que existe um $\bar{\varepsilon}_{0}$ tal que $\operatorname{posto}\left(Q_{\varepsilon}(\lambda)\right) \geqslant \operatorname{posto}(Q(\lambda))$ como queríamos.

Assuma agora que exista uma sequência $\varepsilon_{n} \rightarrow 0$, tal que posto $\left(Q_{\varepsilon_{n}}(\lambda)\right)>\operatorname{posto}(Q(\lambda))=$ $\operatorname{posto}\left(E_{\varepsilon_{n}} Q(\lambda)\right)$. Do Lema IV.2.3 em [35], segue que para cada $n \in \mathbb{N}$ existe $u_{n} \in W\left(\lambda, A_{\varepsilon_{n}}\right)$, $\left\|u_{n}\right\|_{X_{\varepsilon_{n}}}=1$, tal que $\operatorname{dist}\left(u_{n}, E_{\varepsilon_{n}} W\left(\lambda, A_{0}\right)\right)=1$. Da convergência compacta, podemos assumir $u_{n}=Q_{\varepsilon_{n}}(\lambda) u_{n} \stackrel{E}{\longrightarrow} Q(\lambda) u=u$, e assim

$$
1 \leqslant\left\|u_{n}-E_{\varepsilon_{n}} Q(\lambda) u\right\|_{X_{\varepsilon_{n}}}=\left\|Q_{\varepsilon_{n}}(\lambda) u_{n}-E_{\varepsilon_{n}} Q(\lambda) u\right\|_{X_{\varepsilon_{n}}} \rightarrow 0,
$$

uma contradição.

(ii) Seja $u \in W\left(\lambda, A_{0}\right)$ e considere $u_{\varepsilon}=Q_{\varepsilon}(\lambda) u$. Logo $u_{\varepsilon}=Q_{\varepsilon}(\lambda) u \stackrel{E}{\longrightarrow} Q(\lambda) u=u$.

(iii) Dadas as sequências $\varepsilon_{n} \rightarrow 0$ e $u_{n} \in W\left(\lambda, A_{\varepsilon_{n}}\right),\left\|u_{n}\right\|_{X_{\varepsilon_{n}}}=1$, a convergência $Q_{\varepsilon_{n}} \stackrel{C C}{\longrightarrow} Q$ assegura que $u_{n}=Q_{\varepsilon_{n}}(\lambda) u_{n}$ possui subsequência $E$-convergente, digamos $u_{n} \stackrel{E}{\longrightarrow} u$, e assim $u_{n}=Q_{\varepsilon_{n}}(\lambda) u_{n} \stackrel{E}{\longrightarrow} Q(\lambda) u$ mostrando que $u=Q(\lambda) u \in W\left(\mu_{0}, A_{0}\right)$. 


\subsubsection{Continuidade dos semigrupos lineares}

A continuidade do espectro dos operadores $A_{\varepsilon}$ em $\varepsilon=0$ obtida na seção anterior nos permite obter estimativas sobre o comportamento dos semigrupos lineares que por sua vez nos permitem uma análise da dinâmica (não-linear) da equação (2.1). Nosso estudo agora diz respeito a dinâmica da família de equações

$$
\left\{\begin{array}{l}
\dot{u}+A_{\varepsilon} u=F_{\varepsilon}(u), \quad t>0 \\
u(0)=u_{0} \in X_{\varepsilon}
\end{array}\right.
$$

onde os operadores $A_{\varepsilon}$ são operadores setoriais positivos em $X_{\varepsilon}$ satisfazendo a condição de estabilidade

(S) Existem constantes $0<\phi<\frac{\pi}{2}, M \geqslant 1$ e $\omega \in \mathbb{R}$ tal que o setor $\Sigma_{\phi, \omega}=\{\lambda \in \mathbb{C}$ : $|\arg (\lambda-\omega)|<\pi-\phi\}$ está contido no resolvente $\rho\left(A_{\varepsilon}\right)$ para todo $\varepsilon \in[0,1]$ e

$$
\left\|\left(\lambda I-A_{\varepsilon}\right)^{-1}\right\| \leqslant \frac{M}{|\lambda-\omega|}, \forall \lambda \in \Sigma_{\phi, \omega} \text { e } \forall \varepsilon \in[0,1] .
$$

As não linearidades $F_{\varepsilon}: X_{\varepsilon}^{\frac{1}{2}} \rightarrow X_{\varepsilon}$ são supostas limitadas uniformemente em $\varepsilon$, Lipschitz contínuas com constantes de Lipschitz também uniformes em $\varepsilon$ e Frechet diferenciáveis com $F_{\varepsilon}^{\prime}: X_{\varepsilon}^{\frac{1}{2}} \rightarrow \mathscr{L}\left(X_{\varepsilon}^{\frac{1}{2}}, X_{\varepsilon}\right)$.

Observamos que satisfeita a condição de estabilidade (S), para cada $\alpha<\lambda$, existe uma constante $M=M(\alpha)$, tal que

$$
\left\|e^{-A_{\varepsilon} t} u\right\|_{X_{\varepsilon}^{\frac{1}{2}}} \leqslant M t^{-\frac{1}{2}} e^{-\alpha t}\|u\|_{X_{\varepsilon}} .
$$

para todo $t>0$ e todo $\varepsilon \in[0,1]$.

Agora estamos aptos a mostrar uma versão do Teorema de Trotter-Kato para a família de semigrupos $e^{-A_{\varepsilon} t}$.

Teorema 2.14. Sejam $\varepsilon_{n} \stackrel{n \rightarrow \infty}{\longrightarrow} 0,\left\{u_{\varepsilon_{n}}\right\}, u_{\varepsilon_{n}} \in X_{\mathcal{\varepsilon}_{n}}$, e $u_{0} \in X_{0}$ com $u_{\mathcal{\varepsilon}_{n}} \stackrel{E}{\longrightarrow} u_{0}$. Então

$$
\lim _{n \rightarrow \infty}\left\|e^{-A_{\varepsilon_{n}} t} u_{\varepsilon_{n}}-E_{\varepsilon_{n}} e^{-A_{0} t} u_{0}\right\|_{X_{\varepsilon_{n}}^{\frac{1}{2}}}=0,
$$

uniformemente em $\left[t_{1}, t_{2}\right] \subset(0, \infty)$.

Demonstração: Primeiramente observemos que

$$
\begin{aligned}
\left\|e^{-A_{\varepsilon_{n}} t} u_{\varepsilon_{n}}-E_{\varepsilon_{n}} e^{-A_{0} t} u_{0}\right\|_{X_{\varepsilon_{n}}^{\frac{1}{2}}} & \\
& \leqslant\left\|e^{-A_{\varepsilon_{n}} t} u_{\varepsilon_{n}}-e^{-A_{\varepsilon_{n}} t} E_{\varepsilon_{n}} u_{0}\right\|_{X_{\varepsilon_{n}}^{\frac{1}{2}}}+\left\|e^{-A_{\varepsilon_{n}} t} E_{\varepsilon_{n}} u_{0}-E_{\varepsilon_{n}} e^{-A_{0} t} u_{0}\right\|_{X_{\varepsilon_{n}}^{\frac{1}{2}}} \\
& \leqslant M t^{-\frac{1}{2}} e^{-\alpha t}\left\|u_{\varepsilon_{n}}-E_{\varepsilon_{n}} u_{0}\right\|_{X_{\varepsilon_{n}}}+\left\|e^{-A_{\varepsilon_{n}} t} E_{\varepsilon_{n}} u_{0}-e^{-A_{0} t} u_{0}\right\|_{X_{\varepsilon_{n}}^{\frac{1}{2}}}
\end{aligned}
$$


Portanto é suficiente mostrar que

$$
\left\|e^{-A_{\varepsilon_{n}} t} E_{\varepsilon_{n}} u_{0}-E_{\varepsilon_{n}} e^{-A_{0} t} u_{0}\right\|_{X_{\varepsilon_{n}}^{\frac{1}{2}}} \rightarrow 0, \quad n \rightarrow \infty
$$

uniformemente em $\left[t_{1}, t_{2}\right]$.

Observemos que para $u \in X_{0}$, integrando de 0 a $t$ a expressão $\frac{d}{d s}\left[e^{-A_{\varepsilon_{n}}(t-s)} A_{\mathcal{\varepsilon}_{n}}^{-1} E_{\varepsilon_{n}} e^{-A_{0} s} A_{0}^{-1} u\right]$ obtemos

$$
A_{\mathcal{\varepsilon}_{n}}^{-1}\left(E_{\varepsilon_{n}} e^{-A_{0} t}-e^{-A_{\varepsilon_{n} t} t} E_{\varepsilon_{n}}\right) A_{0}^{-1} u=\int_{0}^{t} e^{-A_{\varepsilon_{n}}(t-s)}\left(E_{\varepsilon_{n}} A_{0}^{-1}-A_{\mathcal{\varepsilon}_{n}}^{-1} E_{\varepsilon_{n}}\right) e^{-A_{0} s} u d s
$$

Assim

$$
\begin{aligned}
\left\|A_{\mathcal{E}_{n}}^{-1}\left(E_{\varepsilon_{n}} e^{-A_{0} t}-e^{-A_{\varepsilon_{n}} t} E_{\varepsilon_{n}}\right) A_{0}^{-1} u\right\|_{X_{\varepsilon_{n}}^{\frac{1}{2}}} & \\
& \leqslant \int_{0}^{t}\left\|A_{\varepsilon_{n}}^{\frac{1}{2}} e^{-A_{\varepsilon_{n}}(t-s)}\left(E_{\varepsilon_{n}} A_{0}^{-1}-A_{\varepsilon_{n}}^{-1} E_{\varepsilon_{n}}\right) e^{-A_{0} s} u\right\|_{X_{\varepsilon_{n}}} d s \\
& \leqslant \int_{0}^{t} M e^{\alpha(t-s)}\left\|\left(E_{\varepsilon_{n}} A_{0}^{-1}-A_{\varepsilon_{n}}^{-1} E_{\varepsilon_{n}}\right) e^{-A_{0} s} u\right\|_{X_{\varepsilon_{n}}^{\frac{1}{2}}} d s
\end{aligned}
$$

Observando que $\left\{e^{-A_{0} s} u: s \in[0, t]\right\}$ é um subconjunto compacto de $X_{0}$ segue da convergência compacta $A_{\varepsilon}^{-1} \stackrel{C C}{\longrightarrow} A_{0}^{-1}$, que o integrando converge para 0 uniformemente em $\left[0, t_{1}\right]$, para todo $t_{1}>0$. Logo para todo $v_{0} \in D\left(A_{0}\right)=R\left(A_{0}^{-1}\right)$

$$
\left\|A_{\varepsilon_{n}}^{-1}\left(E_{\varepsilon_{n}} e^{-A_{0} t} v_{0}-e^{-A_{\varepsilon_{n}} t} E_{\varepsilon_{n}} v_{0}\right)\right\|_{X_{\varepsilon_{n}}^{\frac{1}{2}}} \rightarrow 0, \quad n \rightarrow \infty
$$

uniformemente em $\left[0, t_{1}\right]$, para todo $t_{1}>0$. Por densidade (2.16) é válida para todo $v_{0} \in X_{0}$. De fato, seja $z_{0} \in D\left(A_{0}\right)$ tal que $\left\|v_{0}-z_{0}\right\|_{X_{0}}<\delta$. Então

$$
\begin{aligned}
\left\|A_{\varepsilon_{n}}^{-1}\left(E_{\varepsilon_{n}} e^{-A_{0} t} v_{0}-e^{-A_{\varepsilon_{n}} t} E_{\varepsilon_{n}} v_{0}\right)\right\|_{X_{\varepsilon_{n}}^{\frac{1}{2}} \leqslant} & \left\|A_{\varepsilon_{n}}^{-1}\left(E_{\varepsilon_{n}} e^{-A_{0} t}\left(v_{0}-z_{0}\right)-e^{-A_{\varepsilon_{n}} t} E_{\varepsilon_{n}}\left(v_{0}-z_{0}\right)\right)\right\|_{X_{\varepsilon_{n}}^{\frac{1}{2}}} \\
& +\left\|A_{\varepsilon_{n}}^{-1}\left(E_{\varepsilon_{n}} e^{-A_{0} t} z_{0}-e^{-A_{\varepsilon_{n}} t} E_{\varepsilon_{n}} z_{0}\right)\right\|_{X_{\varepsilon_{n}}^{\frac{1}{2}}} \\
\leqslant & \left\|A_{\varepsilon_{n}}^{-1} e^{-A_{\varepsilon_{n} t} t} E_{\varepsilon_{n}}\left(v_{0}-z_{0}\right)\right\|_{X_{\varepsilon_{n}}^{\frac{1}{2}}}+\left\|\left(A_{\varepsilon_{n}}^{-1} E_{\varepsilon_{n}}-E_{\varepsilon_{n}} A_{0}^{-1}\right) e^{-A_{0} t}\left(v_{0}-z_{0}\right)\right\|_{X_{\varepsilon_{n}}^{\frac{1}{2}}} \\
& +\left\|E_{\varepsilon_{n}} A_{0}^{-1} e^{-A_{0} t}\left(v_{0}-z_{0}\right)\right\|_{X_{\varepsilon_{n}}^{\frac{1}{2}}}+\left\|A_{\varepsilon_{n}}^{-1}\left(E_{\varepsilon_{n}} e^{-A_{0} t} z_{0}-e^{-A_{\varepsilon_{n}} t} E_{\varepsilon_{n}} z_{0}\right)\right\|_{X_{\varepsilon_{n}}^{\frac{1}{2}}}
\end{aligned}
$$

Agora se $u \in X_{0}$, temos

$$
\begin{aligned}
\left\|\left(E_{\varepsilon_{n}} e^{-A_{0} t}-e^{-A_{\varepsilon_{n}} t} E_{\varepsilon_{n}}\right) A_{0}^{-1} u\right\|_{X_{\varepsilon_{n}}^{\frac{1}{2}}} & \\
& \leqslant\left\|\left(e^{-A_{\varepsilon_{n}} t} E_{\varepsilon_{n}} A_{0}^{-1}-A_{\varepsilon_{n}}^{-1} e^{-A_{\varepsilon_{n}} t} E_{\varepsilon_{n}}\right) u\right\|_{X_{\varepsilon_{n}}^{\frac{1}{2}}} \\
& +\left\|A_{\varepsilon_{n}}^{-1}\left(E_{\varepsilon_{n}} e^{-A_{0} t}-e^{-A_{\varepsilon_{n}} t} E_{\varepsilon_{n}}\right) u\right\|_{X_{\varepsilon_{n}}^{\frac{1}{2}}}+\left\|\left(A_{\varepsilon_{n}}^{-1} E_{\varepsilon_{n}} e^{-A_{0} t}-E_{\varepsilon_{n}} e^{-A_{0} t} A_{0}^{-1}\right) u\right\|_{X_{\varepsilon_{n}}^{\frac{1}{2}}} .
\end{aligned}
$$

Logo concluímos o resultado através da convergência compacta $A_{\varepsilon}^{-1} \stackrel{C C}{\longrightarrow} A_{0}^{-1}$ e de (2.16). 


\subsubsection{Continuidade dos semigrupos não-lineares}

Recordemos que se $u, v$ são funções contínuas, não negativas, definidas no intervalo $[a, b]$ da reta tais que para algum $\alpha \geqslant 0$

$$
u(t) \leqslant \alpha+\int_{a}^{t} v(s) u(s) d s,
$$

então o Lema de Gronwall garante que

$$
u(t) \leqslant \alpha e^{\int_{a}^{t} v(s) d s},
$$

mostrando em particular que $u \equiv 0$ se $\alpha=0$. Entretanto ao estudarmos estimativas para o semigrupo (linear) associado a equação (2.12) sabemos que

$$
\left\|e^{-A t} u\right\|_{X_{\varepsilon}^{\alpha}} \leqslant M t^{-\alpha}\|u\|_{X_{\varepsilon}}
$$

Neste caso o Lema de Gronwall usual não se aplica. Apresentaremos uma versão generalizada do Lema de Gronwall útil a nossas aplicações. (ver Henry [34])

Lema 2.15 (Desigualdade de Gronwall generalizada). Sejam $t<r, \phi:[t, r] \rightarrow \mathbb{R}^{+}$uma função contínua, $a:[t, r] \rightarrow \mathbb{R}^{+}, b>0$ e $0<\beta \leqslant 1$. Assumamos que

$$
\phi(t) \leqslant a(t)+b \int_{t}^{r}(s-t)^{\beta-1} \phi(s) d s, t \leqslant r .
$$

Então

$$
\phi(t) \leqslant \sum_{k=0}^{\infty}\left(B^{k} a\right)(t)
$$

onde

$$
B^{k} a(t)=\int_{t}^{r} \frac{(b \Gamma(\beta))^{k}}{\Gamma(k \beta)}(s-t)^{k \beta-1} a(s) d s .
$$

Além disso, se a(t) é constante, com $a(t) \equiv a$, então

$$
\phi(t) \leqslant a E_{\beta}\left((b \Gamma(\beta))^{\frac{1}{\beta}}\right)(s-t) \leqslant a c e^{(b \Gamma(\beta))^{\frac{1}{\beta}}(r-t)} .
$$

Se $a(t)=c_{0} \int_{t}^{r}(s-t)^{-\alpha} e^{\rho(s-r)} d s, \rho>0$, então temos

$$
\phi(t) \leqslant \frac{c_{0}}{b}\left[E_{\beta}\left((b \Gamma(\beta))^{\frac{1}{\beta}}\right)(s-t)-1\right] \leqslant \frac{c_{0}}{b}\left[e^{(b \Gamma(\beta))^{\frac{1}{\beta}}(r-t)}-1\right],
$$

$e$, finalmente, se $\psi:[t, r] \rightarrow \mathbb{R}^{+}$é uma função contínua e $a(t)=c_{0} \int_{t}^{r}(s-t)^{-\alpha} e^{\rho s} \psi(s) d s, \rho>0$, então

$$
\phi(t) \leqslant c_{0} c^{\prime} \Gamma(\beta) \int_{t}^{r}(s-t)^{\beta-1} e^{\rho s} e^{d(b \Gamma(\beta))^{\frac{1}{\beta}}(s-t)} \psi(s) d s
$$


onde $\Gamma(\cdot)$ é a função-gama

$$
\Gamma(x)=\int_{0}^{\infty} e^{-t} t^{x-1} d t .
$$

Teorema 2.16. Seja $T_{\varepsilon}(t) u_{\varepsilon}$ a solução da equação (2.12) com $u_{\varepsilon} \in X_{\varepsilon}^{\frac{1}{2}}$. Sejam $\varepsilon_{n} \stackrel{n \rightarrow \infty}{\longrightarrow} 0$, $\left\{u_{\varepsilon_{n}}\right\}, u_{\varepsilon_{n}} \in X_{\varepsilon_{n}}^{\frac{1}{2}}, u_{0} \in X_{0}^{\frac{1}{2}}$ com $u_{\varepsilon_{n}} \stackrel{E}{\longrightarrow} u_{0}$. Dado $T \in(0, \infty)$, se $t_{n} \stackrel{n \rightarrow \infty}{\longrightarrow} t \in[0, T]$ então

$$
\left\|T_{\mathcal{E}}\left(t_{n}\right) u_{\varepsilon_{n}}-E_{\varepsilon_{n}} T_{0}(t) u_{0}\right\|_{X_{\varepsilon}^{\frac{1}{2}}} \rightarrow 0, \quad n \rightarrow \infty .
$$

Demonstração: Para $t \in[0, T]$ temos pela Fórmula da Variação das Constantes,

$$
\begin{aligned}
T_{\mathcal{\varepsilon}}(t) u_{\varepsilon_{n}}-E_{\varepsilon_{n}} T_{0}(t) u_{0}=e^{-A_{\varepsilon} t} u_{\varepsilon_{n}}-E_{\varepsilon_{n}} e^{-A_{0} t} u_{0} & +\int_{0}^{t} e^{-A_{\varepsilon_{n}}(t-s)}\left[F_{\varepsilon}\left(T_{\varepsilon}(s) u_{\varepsilon_{n}}\right)-E_{\varepsilon_{n}} F_{0}\left(T_{0}(s) u_{0}\right)\right] d s \\
& +\int_{0}^{t}\left[e^{-A_{\varepsilon_{n}}(t-s)} E_{\varepsilon_{n}}-E_{\varepsilon_{n}} e^{-A_{0}(t-s)}\right] F_{0}\left(T_{0}(s) u_{0}\right) d s .
\end{aligned}
$$

Da continuidade dos semigrupos lineares obtemos,

$$
\begin{aligned}
\left\|e^{-A_{\varepsilon} t} u_{\varepsilon_{n}}-E_{\varepsilon_{n}} e^{-A_{0} t} u_{0}\right\|_{X_{\varepsilon}^{\frac{1}{2}}} & \leqslant\left\|e^{-A_{\varepsilon} t} u_{\varepsilon_{n}}-E_{\varepsilon_{n}} e^{-A_{\varepsilon} t} u_{0}\right\|_{X_{\varepsilon}^{\frac{1}{2}}}+\left\|e^{-A_{\varepsilon} t} E_{\varepsilon_{n}} u_{0}-E_{\varepsilon_{n}} e^{-A_{0} t} u\right\|_{X_{\varepsilon}^{\frac{1}{2}}} \\
& \leqslant M e^{-\alpha t}\left\|u_{\varepsilon_{n}}-E_{\varepsilon_{n}} u_{0}\right\|_{X_{\varepsilon}^{\frac{1}{2}}}+o(1) .
\end{aligned}
$$

Observemos que $\left.\left\{F_{0}\left(T_{0}(s) u_{0}\right)\right): s \in[0, t]\right\}$ é um subconjunto compacto de $X_{0}$, de modo que podemos afirmar que a integral

$$
\left.\int_{0}^{t} \|\left[e^{-A_{\varepsilon}(t-s)} E_{\varepsilon_{n}}-E_{\varepsilon_{n}} e^{-A_{0}(t-s)}\right] F_{0}\left(T_{0}(s) u_{0}\right)\right) \|_{X_{\varepsilon}^{\frac{1}{2}}} d s \rightarrow 0, \quad \varepsilon \rightarrow 0
$$

Além disso, se $L$ é a constante de Lipschitz das $F_{\varepsilon}$, então

$$
\begin{aligned}
\int_{0}^{t} \| e^{-A_{\varepsilon}(t-s)}\left[F_{\varepsilon}\left(T_{\mathcal{E}}(s) u_{\varepsilon_{n}}\right)-\right. & \left.E_{\varepsilon_{n}} F_{0}\left(T_{0}(s) u_{0}\right)\right] \|_{X_{\varepsilon}^{\frac{1}{2}}} d s \\
& \leqslant M L \int_{0}^{t}(t-s)^{-\frac{1}{2}} e^{-\alpha(t-s)}\left\|T_{\varepsilon}(s) u_{\varepsilon_{n}}-E_{\varepsilon_{n}} T_{0}(s) u_{0}\right\|_{X_{\varepsilon}^{\frac{1}{2}}} d s
\end{aligned}
$$

Logo,

$$
\begin{aligned}
e^{\alpha t}\left\|T_{\varepsilon_{n}}(t) u_{\varepsilon_{n}}-E_{\varepsilon_{n}} T_{0}(t) u_{0}\right\|_{X_{\varepsilon}^{\frac{1}{2}}} \leqslant & M\left\|u_{\varepsilon_{n}}-E_{\varepsilon_{n}} u_{0}\right\|_{X_{\varepsilon}^{\frac{1}{2}}}+o(1) e^{\alpha t} \\
& +M L \int_{0}^{t}(t-s)^{-\frac{1}{2}} e^{\alpha s}\left\|T_{\varepsilon_{n}}(s) u_{\varepsilon_{n}}-E_{\varepsilon_{n}} T_{0}(s) u_{0}\right\|_{X_{\varepsilon}^{\frac{1}{2}}} d s .
\end{aligned}
$$

e as duas primeiras parcelas do lado direito da última desigualdade podem ser majoradas por $\tilde{M} v(\varepsilon), \operatorname{com} \tilde{M}=\tilde{M}(T)$ e $v(\varepsilon) \stackrel{\varepsilon \rightarrow 0}{\longrightarrow} 0$.

Agora, colocando $\varphi_{\varepsilon_{n}}(t)=e^{\omega t}\left\|T_{\varepsilon_{n}}(t) u_{\varepsilon_{n}}-E_{\varepsilon_{n}} T_{0}(t) u_{0}\right\|_{X_{\varepsilon}^{\frac{1}{2}}}$, temos para $n$ suficientemente grande

$$
\varphi(t)_{\varepsilon_{n}} \leqslant \tilde{M}+M L \int_{0}^{t}(t-s)^{-\frac{1}{2}} \varphi(s) d s \quad \text { em }(0, T) .
$$


Do Lema 2.15 segue então que

$$
\varphi_{\varepsilon_{n}}(t) \leqslant \tilde{M}_{1} v\left(\varepsilon_{n}\right) \rightarrow 0, \quad n \rightarrow \infty,
$$

onde $\tilde{M}_{1}=(M, \tilde{M}, L, T)$ é uma constante independente de $\varepsilon$.

\subsection{Continuidade dos atratores}

As hipóteses da seção 2.1.1 garantem que os semigrupos $\left\{T_{\mathcal{\varepsilon}}(t) ; t \geqslant 0\right\}$ associados a familia de equações (2.12) possuem atratores globais $\mathscr{A}_{\varepsilon}$ em $X_{\varepsilon}^{\frac{1}{2}}, \varepsilon \in[0, \bar{\varepsilon}]$. Além disso, garantem também sua limitação uniforme,

$$
\sup _{\varepsilon \in[0, \bar{\varepsilon}]} \sup _{w \in \mathscr{A}_{\varepsilon}}\|w\|_{X_{\varepsilon}^{\frac{1}{2}}}<\infty .
$$

Nossa Definição 2.17 de semicontinuidade superior e inferior da família de atratores $\mathscr{A}_{\varepsilon}$ requer um sentido apropriado levando em conta a variação dos espaços funcionais.

Definição 2.17. Consideremos uma família de conjuntos $\left\{J_{\varepsilon} \subset X_{\varepsilon}\right\}$.

A família $\left\{J_{\varepsilon}\right\}$ é semicontínua superiormente em $\varepsilon=0$ se $\lim _{\varepsilon \rightarrow 0} \operatorname{dist}\left(J_{\varepsilon}, E_{\varepsilon} J_{0}\right)=0$.

A família $\left\{J_{\varepsilon}\right\}$ é semicontínua inferiormente em $\varepsilon=0$ se $\lim _{\varepsilon \rightarrow 0} \operatorname{dist}\left(E_{\varepsilon} J_{0}, J_{\varepsilon}\right)=0$.

A familia $\left\{J_{\varepsilon}\right\}$ é contínua em $\lambda=\lambda_{0}$ se for semicontínua superior e inferiormente.

\section{Lema 2.18.}

(i) A família $\left\{J_{\varepsilon}\right\}$ é semicontínua superiormente em $\varepsilon=\varepsilon_{0}$ se toda seqüência $\left\{u_{\mathcal{\varepsilon}_{n}}\right\}_{n \in \mathbb{N}}$, com $u_{\varepsilon_{n}} \in J_{\mathcal{E}_{n}}, \varepsilon_{n} \rightarrow 0$, possui uma subseqüência $E$-convergente para um elemento de $J_{0}$.

(ii) Se $J_{0}$ é compacto e para todo $u \in J_{0}$, existe uma seqüência $\left\{u_{\varepsilon_{n}}\right\}_{n \in \mathbb{N}}$ com $u_{\varepsilon_{n}} \in J_{\varepsilon_{n}}, \varepsilon_{n} \rightarrow 0$, tal que $u_{\varepsilon_{n}} \stackrel{E}{\longrightarrow}$ u, então a família $\left\{J_{\mathcal{\varepsilon}}\right\}$ é semicontínua inferiormente em 0 .

Com os resultados das seções anteriores

Teorema 2.19. A família de atratores $\left\{\mathscr{A}_{\varepsilon}: \varepsilon \in\left[0, \varepsilon_{0}\right]\right\}$ é semicontínua superiormente em $\varepsilon=0$, ou seja,

$$
\lim _{\varepsilon \rightarrow 0} \sup _{u_{\varepsilon} \in \mathscr{A}_{\varepsilon}} \inf _{u_{0} \in \mathscr{A}_{0}}\left\|u_{\varepsilon}-E_{\varepsilon} u_{0}\right\|_{X_{\varepsilon}^{\frac{1}{2}}}=0 .
$$

Demonstração: Consideremos as seqüencias $\varepsilon_{n} \rightarrow 0,\left\{u_{\varepsilon_{n}}\right\}, u_{\varepsilon_{n}} \in \mathscr{A}_{\varepsilon_{n}}$. Por hipótese temos que

$$
\sup _{\varepsilon \in[0, \bar{\varepsilon}] w \in \mathscr{A}_{\varepsilon}} \sup _{X_{\varepsilon}}\|w\|_{\frac{1}{2}}<\infty
$$




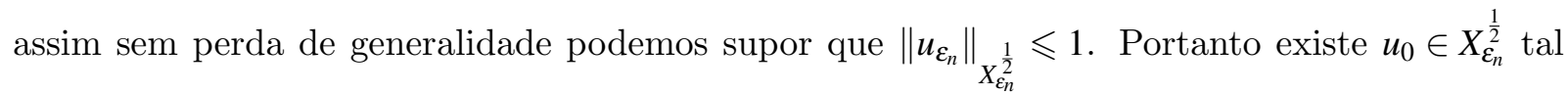
que $u_{\varepsilon_{n}} \stackrel{E}{\longrightarrow} u_{0}$.

Resta-nos mostrar que $u_{0} \in \mathscr{A}_{0}$. Para tanto, é suficiente mostrarmos que existe uma órbita completa limitada por $u_{0}$. Da invariância dos atratores $\mathscr{A}_{\varepsilon_{n}}$ para cada $n \in \mathbb{N}$, existe uma órbita completa e limitada

$$
\begin{aligned}
\psi_{\varepsilon_{n}}: & \mathbb{R} \rightarrow X_{\varepsilon_{n}}^{\frac{1}{2}} \\
t & \mapsto \psi_{\varepsilon_{n}}\left(t, u_{\varepsilon_{n}}\right)
\end{aligned}
$$

por $u_{\varepsilon_{n}}$. Para $t \geqslant 0$, segue da continuidade dos semigrupos não-lineares que

$$
\psi_{\varepsilon_{n}}\left(t, u_{\varepsilon_{n}}\right)=T_{\varepsilon_{n}}(t) u_{\varepsilon_{n}} \rightarrow T_{0}(t) u_{0} .
$$

Por outro lado para $t<0$, construímos uma órbita por $u_{0}$ do seguinte modo:

Dado $k \in \mathbb{Z}^{+}$, suponha sem perda de generalidade que $0<\varepsilon_{n}<\varepsilon_{0}$. Se $t \in(-k,-k+1]$, consideramos a seqüência $\left\{\psi_{\varepsilon_{n}}\left(-k, u_{\varepsilon_{n}}\right)\right\}_{n \in \mathbb{N}}$ em $\bigcup_{\varepsilon \in(0, \bar{\varepsilon}]} \mathscr{A}_{\mathcal{\varepsilon}}$. Novamente existe um elemento que denotaremos por $\tilde{\psi}_{0}\left(-k, u_{0}\right) \in X_{0}^{\frac{1}{2}}$ tal que $\psi_{\varepsilon_{n}}\left(-k, u_{\varepsilon_{n}}\right) \stackrel{X_{\varepsilon_{n}}}{\longrightarrow} \tilde{\psi}_{0}(-k, u)$.

Portanto para $t \in(-k,-k+1]$,

$$
\psi_{\varepsilon_{n}}\left(t, u_{\varepsilon_{n}}\right)=\psi_{\varepsilon_{n}}\left(t+k, \psi_{\varepsilon_{n}}\left(-k, u_{\varepsilon_{n}}\right)\right)=T_{\varepsilon_{n}}(t+k) \psi_{\varepsilon_{n}}\left(-k, u_{\varepsilon_{n}}\right) \rightarrow T_{0}(t+k) \psi_{0}\left(-k, u_{0}\right):=\tilde{\psi}_{0}\left(t, u_{0}\right) .
$$

Finalmente, definindo

$$
\psi_{0}(t, u)= \begin{cases}T_{0}(t) u_{0}, & \text { para } t \geqslant 0 \\ \tilde{\psi}_{0}\left(t, u_{0}\right), & \text { para } t<0\end{cases}
$$

vemos que $\psi_{0}\left(t, u_{0}\right)$ é uma órbita completa limitada por $u_{0}$. Agora o resultado segue do Lema 2.18 .

\subsubsection{Continuidade dos conjuntos de equilíbrio}

Antes de descrevermos a estrutura do atrator global iniciemos estudando a continuidade dos elementos mais simples que vivem no atrator, as soluções de equilíbrio. As soluções de equilíbrio são aquelas que independem do tempo, ou seja, as soluções do problema elíptico

$$
A_{\mathcal{\varepsilon}} u-F_{\varepsilon}(u)=0, \quad\left(\text { resp. } A_{0} u-F_{0}(u)=0\right) \text {. }
$$

O fato dos operadores $A_{\varepsilon}$ serem setoriais com resolvente compacto entra de forma crucial na prova da continuidade da dinâmica próxima a equilíbrios, pois a linearização do lado direito da equação (2.12) terá somente um número finito de autovalores com parte real positiva. 
Definição 2.20. Seja $\mathscr{E}_{\varepsilon}=\left\{u_{\varepsilon}^{*} \in X_{\varepsilon}: A_{\varepsilon} u_{\varepsilon}^{*}-F_{\varepsilon}\left(u_{\varepsilon}^{*}\right)=0\right\}, \varepsilon \in[0,1]$. Uma solução de equilíbrio $u_{\mathcal{\varepsilon}}^{*} \in \mathscr{E}_{\varepsilon}$ é hiperbólica se o espectro $\sigma\left(A_{\varepsilon}-F_{\varepsilon}^{\prime}\left(u_{\varepsilon}^{*}\right)\right)$ do operador $A_{\varepsilon}-F_{\varepsilon}^{\prime}\left(u_{\varepsilon}^{*}\right)$ é disjunto do eixo imaginário, isto é, $\sigma\left(A_{\varepsilon}-F_{\varepsilon}^{\prime}\left(u_{\varepsilon}^{*}\right)\right) \cap i \mathbb{R}=\emptyset$.

Neste ponto fazemos também a seguinte hipótese:

(CC) A família $\left\{A_{\varepsilon}^{-1} F_{\varepsilon}\right\}_{\varepsilon \in(0,1]}$ converge compactamente para o operador $A_{0}^{-1} F_{0}$.

Agora a semicontinuidade superior dos conjuntos $\mathscr{E}_{\varepsilon}$ em $\varepsilon=0$ segue como uma consequência desta convergência compacta.

Lema 2.21. Dada uma seqüencia $\stackrel{n \rightarrow \infty}{\longrightarrow} 0$, seja $\left\{u_{\mathcal{E}_{n}}^{*}\right\}, u_{\mathcal{E}_{n}}^{*} \in \mathscr{E}_{\mathcal{E}_{n}}$. Então a menos de subseqüências existe um equilíbrio $u_{0}^{*} \in \mathscr{E}_{0}$ tal que

$$
\lim _{n \rightarrow \infty}\left\|u_{\varepsilon_{n}}^{*}-E_{\varepsilon} u_{0}^{*}\right\|_{X_{\varepsilon}^{\frac{1}{2}}}=0 .
$$

Demonstração: Observemos que $\mathscr{E}_{\varepsilon_{n}} \subset \mathscr{A}_{\varepsilon_{n}}$, logo $\sup _{\varepsilon_{n}}\left\|F_{\varepsilon_{n}}\left(u_{\varepsilon_{n}}^{*}\right)\right\|_{X_{\varepsilon_{n}}^{\frac{1}{2}}}<\infty$. Por hipótese segue que a seqüencia $\left\{u_{\mathcal{E}_{n}}^{*}=A_{\mathcal{\varepsilon}_{n}}^{-1} F_{\mathcal{E}_{n}}\left(u_{\mathcal{E}_{n}}^{*}\right)\right\}$ é $E$-relativamente compacta, e portanto existe $u_{0}^{*} \in X_{0}^{\frac{1}{2}}$ tal que $\left\|u_{\varepsilon_{n}}^{*}-E_{\varepsilon} u_{0}^{*}\right\|_{X_{\varepsilon}^{\frac{1}{2}}} \rightarrow 0, \quad n \rightarrow \infty$. Novamente pela convergência compacta dos resolventes e a continuidade de $F_{\varepsilon}$ segue que $u_{0}^{*}=A_{0}^{-1} F_{0}\left(u_{0}^{*}\right) \in \mathscr{E}_{0}$.

Teorema 2.22. Se $u_{0}^{*} \in \mathscr{E}_{0}$ é um ponto de equilíbrio tal que $0 \notin \sigma\left(A_{0}-F_{0}^{\prime}\left(u_{0}^{*}\right)\right)$ então, $u_{0}^{*}$ é uma solução isolada e $\left|\mathrm{D}\left(u_{0}^{*}, I-A_{0}^{-1} F_{0}^{\prime}\left(u_{0}^{*}\right)\right)\right|=1$.

Demonstração: Observemos que $u_{0}^{*} \in \mathscr{E}_{0}$ se e somente é ponto fixo do operador compacto $-A_{0}^{-1} F_{0}(\cdot): U \rightarrow U$. Também, $0 \notin \sigma\left(A_{0}+F_{0}^{\prime}\left(u^{*}\right)\right)$ se e somente se $1 \notin \sigma\left(I-A_{0}^{-1} F_{0}^{\prime}\left(u^{*}\right)\right)$. E segue que existe uma constante $\eta>0$ tal que $\left\|v+A_{0}^{-1} F_{0}\left(u^{*}\right) v\right\|_{X_{0}} \geqslant 2 \eta\|v\|_{X_{0}}$. Se escrevermos

$$
A_{0}^{-1} F_{0}\left(u^{*}+v\right)-A_{0}^{-1} F_{0}\left(u^{*}\right)-A_{0}^{-1} F_{0}^{\prime}\left(u^{*}\right) v=w\left(u^{*}, v\right), \quad \frac{\left\|w\left(u^{*}, v\right)\right\|_{X_{0}}}{\|v\|_{X_{0}}} \stackrel{v \rightarrow 0}{\longrightarrow} 0
$$

existe $r>0$ tal que $\left\|w\left(u^{*}, v\right)\right\|_{X_{0}} \leqslant \alpha\|v\|_{X_{\varepsilon}}$ para $\|v\|_{X_{0}} \leqslant r$. Então, para $\left\|u^{*}-u\right\|_{X_{0}} \leqslant r$

$$
\left\|u-A_{0}^{-1} F_{0}(u)\right\|_{X_{0}} \geqslant\left\|u-u^{*}-A_{0}^{-1} F_{0}^{\prime}\left(u^{*}\right)\left(u^{*}-u\right)\right\|_{X_{0}}-\left\|w\left(u^{*}, u-u^{*}\right)\right\|_{X_{0}} \geqslant \eta\left\|u-u^{*}\right\| .
$$

Portanto $u^{*}$ é um ponto de equilíbrio isolado. O restante da prova decorre do Teorema 1.51.

Corolário 2.23. Se $u_{0}^{*} \in \mathscr{E}_{0}$ é uma equilíbrio hiperbólico então, $u_{0}^{*}$ é um equilíbrio isolado e $\left|\mathrm{D}\left(u^{*}, I+A_{0}^{-1} F_{0}^{\prime}\left(u_{0}^{*}\right)\right)\right|=1$. 
Proposição 2.24. Se $\mathscr{E}_{0}$ é um conjunto discreto, então $\mathscr{E}_{0}$ é um conjunto finito. Se $0 \notin \sigma\left(A_{0}+\right.$ $\left.F_{0}^{\prime}\left(u_{0}^{*}\right)\right)$ para todo $u_{0}^{*} \in \mathscr{E}_{0}$, então $\mathscr{E}_{0}$ é um conjunto finito. Além disso, se todos seus pontos são hiperbólicos esse número é ímpar.

Demonstração: Observemos primeiramente que para todo $u_{0}^{*} \in \mathscr{E}_{0}, u_{0}^{*}+A_{0}^{-1} F_{0}\left(u_{0}^{*}\right)=0$. Considerando $K=\sup _{u \in X_{0}^{\frac{1}{2}}}\left\{\left\|F_{0}(u)\right\|_{X_{0}^{\frac{1}{2}}}\right\}$, o operador $-A_{0}^{-1} F_{0}(\cdot)$ leva a bola $B_{X_{0}^{\frac{1}{2}}}\left(0,\left\|A^{-1}\right\| K\right)$ nela mesma.

Pelo Teorema do Ponto Fixo de Schauder $D\left(I+A_{0}^{-1} F_{0}(\cdot), B\left(0,\left\|A^{-1}\right\| K\right)=1\right.$, e portanto, existe ao menos um ponto fixo $u_{0}^{*}$ de $-A_{0}^{-1} F_{0}(\cdot)$ na bola $\left.B_{X_{0}^{\frac{1}{2}}}\left(0,\left\|A^{-1}\right\| K\right)\right)$; isto é,

$$
\left.I u^{*}+A^{-1} F_{0}\left(u^{*}\right)=0 \operatorname{com} u^{*} \in B_{X_{0}^{\frac{1}{2}}}\left(0,\left\|A^{-1}\right\| K\right)\right) .
$$

Sendo o operador $-A_{0}^{-1} F_{0}(\cdot): X_{0}^{\frac{1}{2}} \longrightarrow X_{0}^{\frac{1}{2}}$ compacto, temos $\mathscr{E}_{0}=\left\{u_{0}^{*}: A_{0} u_{0}^{*}+F_{0}\left(u_{0}^{*}\right)=0\right\}$ é compacto em $X_{0}^{\frac{1}{2}}$. Além disso, pelo Teorema 2.22 todo ponto fixo $u_{0}^{*}$ é isolado. Se o número de pontos fixos fosse infinito, existiria uma seqüencia $\left\{u_{n}^{*}\right\}_{n \in \mathbb{N}}$, tal que a seqüencia $-A_{0}^{-1} F_{0}\left(u_{n}^{*}\right)=u_{n}^{*} \rightarrow u_{0}^{*}$ para alguma subseqüência $n \in \mathbb{N}^{\prime} \subset \mathbb{N}$, contradizendo o fato de ser $u_{0}^{*}$ isolado.

O Próximo Teorema garante a semicontinuidade inferior da família de equilíbrios $\mathscr{E}_{\varepsilon}$.

Teorema 2.25 (G. Vainikko, [49]). Sejam $\Omega_{\varepsilon} \subset X_{\varepsilon}$ abertos e limitados e $T_{\varepsilon}: \bar{\Omega}_{\varepsilon} \rightarrow X$. Suponhamos válidas as seguintes hipóteses

(i) Os operadores $T_{\varepsilon}: \bar{\Omega}_{\varepsilon} \rightarrow X$ são completamente contínuos e além disso, $T_{0}$ não possui ponto fixo em $\partial \Omega_{0}$ e $D\left(I-T_{0}, \Omega, 0\right) \neq 0$;

(ii) Os abertos $\Omega_{\varepsilon}$ são tais que

- para todo $u_{0} \in \bar{\Omega}$ existe uma família $\left\{u_{\varepsilon}\right\}, u_{\varepsilon} \in \Omega_{\varepsilon}$ tal que $u_{\varepsilon} \stackrel{E}{\longrightarrow} u_{0}$;

- se $u_{\varepsilon} \in \bar{\Omega}_{\varepsilon}$ é tal que $u_{\varepsilon} \stackrel{E}{\longrightarrow} u_{0}$ então $u_{0} \in \bar{\Omega}_{0}$;

- se $u_{\varepsilon} \in \partial \Omega_{\varepsilon}$, é tal que $u_{\varepsilon} \stackrel{E}{\longrightarrow} u_{0}$ então $u_{0} \in \partial \Omega$.

(iii) $T_{\varepsilon} \stackrel{C C}{\longrightarrow} T_{0}$.

Então existe $\varepsilon_{0}$ tal que para $0<\varepsilon<\varepsilon_{0}$ os operadores $T_{\varepsilon}$ possuem ponto fixo $u_{\varepsilon}^{*}$ em $\bar{\Omega}_{\varepsilon}$. Além disso, qualquer família $\left\{u_{\varepsilon}^{*}\right\}$ de pontos fixos de $T_{\varepsilon}$ é $E$-compacta e seus E-limites são pontos fixos de $T_{0}$ em $\Omega_{0}$. Em particular se $T_{0}$ possui somente um único ponto fixo $u_{0}^{*}$ de índice não nulo, então $\left\|u_{\varepsilon}^{*}-E_{\varepsilon} u_{0}^{*}\right\|_{X_{\varepsilon}} \rightarrow 0$. 
Proposição 2.26. Assuma que $u_{0}^{*} \in \mathscr{E}_{0}$ seja um equilíbrio hiperbólico. Então existem $\varepsilon_{0}$ e $\boldsymbol{\delta}>0$ tais que para $0<\varepsilon \leqslant \varepsilon_{0}$ existe ao menos um equilibrio $u_{\varepsilon}^{*} \in \mathscr{E}_{\varepsilon} \bigcap\left\{w_{\varepsilon}:\left\|w_{\varepsilon}-E_{\varepsilon} u_{0}^{*}\right\|_{X_{\varepsilon}^{\frac{1}{2}}} \leqslant \delta\right\}$. Além disso, $\left\|u_{\varepsilon}^{*}-E_{\varepsilon} u_{0}^{*}\right\|_{X_{\varepsilon}^{\frac{1}{2}}} \rightarrow 0$.

Demonstração: Como no Corolário 2.23 existe uma bola $B\left(u_{0}^{*}, \delta\right)$ tal que não existem outros pontos fixos nela exceto $u_{0}^{*}$, e assim $\left|D\left(u_{0}^{*}, I+A^{-1} F_{0}(\cdot)\right)\right|=1$. Agora colocando $\Omega_{0}=B\left(u_{0}^{*}, \delta\right), \Omega_{\varepsilon}=$ $B\left(E_{\varepsilon} u_{0}^{*}, \delta\right)$ e $T_{0}=I+A^{-1} F_{0}(\cdot), T_{\varepsilon}=I+A_{\varepsilon_{n}}^{-1} F_{\varepsilon}(\cdot)$ no Teorema 2.25 suas hipóteses ficam claramente satisfeitas (exceto possivelmente a condição (ii)), e portanto existe pelo menos um ponto fixo $u_{\varepsilon}^{*}$ na bola $B\left(E_{\varepsilon_{n}} u_{0}^{*}, \delta\right)$. Ainda $u_{\varepsilon}^{*} \stackrel{E}{\longrightarrow} u_{0}^{*}$.

Verificação da condição (ii) do Teorema 2.25

Sejam $\Omega_{0}$ e $\Omega_{\varepsilon}$ e $u_{0}^{*}$ como antes.

- Se $u_{0} \in \bar{\Omega}_{0}$, como $\left\|E_{\varepsilon} u_{0}-E_{\varepsilon} u_{0}^{*}\right\| \rightarrow\left\|u_{0}-u_{0}^{*}\right\| \leqslant \delta$ temos que $E_{\varepsilon} u_{0} \in \Omega_{\varepsilon}$ para $\varepsilon$ suficientemente pequeno e ainda $u_{\varepsilon}=E_{\varepsilon} u_{0} \stackrel{E}{\longrightarrow} u_{0}$.

- Se $u_{\varepsilon} \in \bar{\Omega}_{\varepsilon}$ é tal que $u_{\varepsilon} \stackrel{E}{\longrightarrow} u_{0}$, basta observar que $\left\|u_{0}-u_{0}^{*}\right\| \leftarrow\left\|E_{\varepsilon} u_{0}-E_{\varepsilon} u_{0}^{*}\right\| \leqslant\left\|E_{\varepsilon} u_{0}-u_{\varepsilon}\right\|+$ $\left\|u_{\varepsilon}-E_{\varepsilon} u_{0}^{*}\right\|$.

- Novamente se $u_{\varepsilon} \in \partial \Omega_{\varepsilon}$, é tal que $u_{\varepsilon} \stackrel{E}{\longrightarrow} u_{0}$ basta observar que, $0 \leqslant||\left|u_{0}^{*}-u_{0} \|-\delta\right| \leftarrow$ ||$\left|E_{\varepsilon} u_{0}^{*}-E_{\varepsilon} u_{0}\left\|-\delta|\leqslant||| E_{\varepsilon} u_{0}^{*}-u_{\varepsilon}\right\|+\left\|u_{\varepsilon}-E_{\varepsilon} u_{0}\right\|-\delta\right|=\left\|u_{\varepsilon}-E_{\varepsilon} u_{0}\right\| \rightarrow 0$.

2.2.2 Linearização em torno de um ponto de equilíbrio

Nesta seção mostraremos que existem muitas outras soluções que estão nos atratores $\mathscr{A}_{\varepsilon}$ além das soluções de equilíbrio. Da caracterização via Fórmula da Variação das Constantes para uma solução da equação (2.1) e nos fazendo valer do Lema de Gronwall generalizado, é fácil ver que para qualquer $u_{0} \in X_{\varepsilon}^{\frac{1}{2}}, \varepsilon \in[0,1]$, a solução $T_{\varepsilon}(t) u_{0}$ por $u_{0}$ permanece limitada, ou seja,

$$
\sup _{t \geqslant 0}\left\|T_{\varepsilon}(t) u_{0}\right\|_{X_{\varepsilon}^{\frac{1}{2}}}<\infty
$$

Logo se mostramos que existe uma solução global $T_{\varepsilon}(\cdot) u_{0}: \mathbb{R} \longrightarrow X_{\varepsilon}^{\frac{1}{2}}$ por $u_{0}$ que permanece limitada, então ela deve necessariamente pertencer ao atrator $\mathscr{A}_{\mathcal{E}}$. Assim é suficiente exibir uma solução por $u_{0}$ que pode ser continuada para todo $t<0$ e que permaneça limitada para obter uma solução no atrator $\mathscr{A}_{\varepsilon}$. 
Definição 2.27. Para $\varepsilon \in\left[0, \varepsilon_{0}\right]$, o conjunto instável de $u_{\varepsilon}^{*} \in \mathscr{E}_{\varepsilon}$ é o conjunto $\mathrm{W}^{u}\left(u_{\varepsilon}^{*}\right)=\left\{\eta \in X_{\varepsilon}^{\frac{1}{2}}\right.$ : existe uma solução $u(t, \eta)$ definido para todo $t \leqslant 0$ tal que $\left.u(t, \eta) \stackrel{t \rightarrow-\infty}{\longrightarrow} u_{\varepsilon}^{*}\right\}$.

Antes de darmos mais detalhes sobre como construir $\mathrm{W}^{u}\left(u_{0}^{*}\right)$ ao menos em uma vizinhança de $u_{0}^{*}$, observemos o que ocorre com a equação (2.12) em uma vizinhança de um de equilíbrio $x_{0}^{*} \in \mathscr{E}_{0}$. Fazendo a mudança de variáveis $v=u-u_{0}^{*}$, olhemos por um instante a equação

$$
\left\{\begin{array}{l}
\dot{v}+\bar{A}_{0} v=F_{0}\left(v+u_{0}^{*}\right)-F_{0}\left(u_{0}^{*}\right)-F_{0}^{\prime}\left(u_{0}^{*}\right) v \\
v(0)=u_{0}-u_{0}^{*}=v_{0}
\end{array}\right.
$$

onde, $\bar{A}_{0}=\left(A_{0}-F_{0}^{\prime}\left(u_{0}^{*}\right)\right)$.

Esta equação é equivalente a equação (2.12) neste novo sistema de coordenadas. Entretanto podemos observar que para $v$ muito pequeno, a parte não-linear é também muito pequena. É natural então estudarmos a equação (2.12) linearizada em uma vizinhança de $u_{0}^{*}$ e estudar o quão estável ela permanece por pequenas pertubações, ou seja, estudar a equação

$$
\left\{\begin{array}{l}
\dot{v}+\bar{A}_{0} v=0 \\
v(0)=v_{0}
\end{array}\right.
$$

Agora denotemos por $\sigma_{\varepsilon}^{+}=\left\{\mu \in \sigma\left(-\bar{A}_{\varepsilon}\right): \operatorname{Re} \mu>0\right\}, \varepsilon \in\left[0, \varepsilon_{0}\right]$ e definimos $Q_{0}^{+}\left(\sigma^{+}\right)$a projeção determinada por $\sigma_{0}^{+}$, isto é,

$$
Q_{0}^{+}\left(\sigma_{0}^{+}\right)=\frac{1}{2 \pi i} \int_{\Gamma^{+}}\left(\mu+\bar{A}_{0}\right)^{-1} d \mu,
$$

onde $\Gamma^{+}$é uma curva em $\left\{\mu \in \rho\left(-A_{0}\right): \operatorname{Re} \mu>0\right\}$, que envolve $\sigma_{0}^{+}$.

Para cada $v_{0} \in Q_{0} X_{0}^{\frac{1}{2}}$, a solução $v\left(t, v_{0}\right)$ de $(2.12)$ existe para todo $t \leqslant 0$ e $v\left(t, v_{0}\right) \rightarrow 0$ quando $t \rightarrow-\infty$ e ainda $v+u_{*} \rightarrow u_{*}$ quando $t \rightarrow-\infty$. Este fato serve como inspiração para o que queremos fazer. Quando perturbamos (2.12) com uma não-linearidade muito pequena, devemos observar soluções de (2.21) que existem para todo $t \leqslant 0$. Naturalmente, o dado inicial para o qual tais soluções existem não estará no subespaço linear $Q_{0}^{+} X_{0}^{\frac{1}{2}}$, mas em uma variedade não-linear próxima.

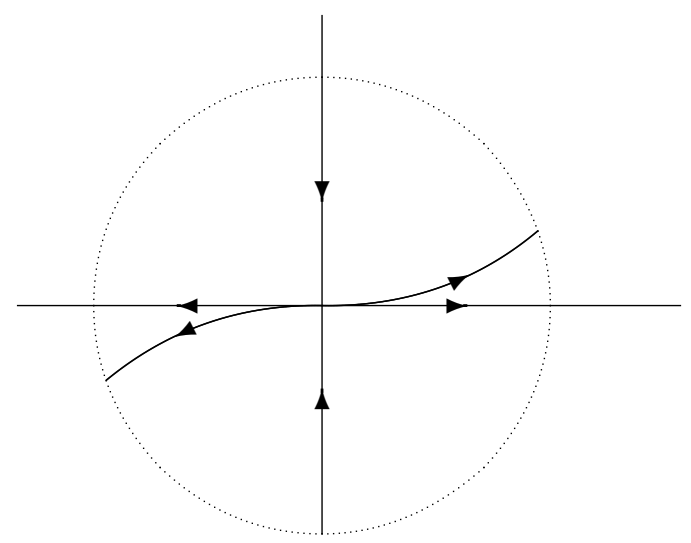


Teorema 2.28. Suponhamos que $u_{0}^{*} \in \mathscr{E}_{0}$ seja um equilíbrio hiperbólico do problema (2.12) com $\varepsilon=0$. Então existem uma vizinhança $U$ de $x_{0}^{*}$ em $X_{0}^{\frac{1}{2}}$ e uma aplicação $S_{0}: Q_{0}^{+} U \longrightarrow\left(I-Q_{0}^{+}\right) U$, tais que o conjunto instável local $W_{\delta}^{u}\left(u_{0}^{*}\right)$ de $u_{0}^{*}$ é dado por

$$
W_{\delta}^{u}\left(u_{0}^{*}\right)=:\left\{w \in W^{u}\left(u_{0}^{*}\right):\left\|w-u_{0}^{*}\right\|_{X_{0}^{\frac{1}{2}}}<\delta\right\}=\left\{\left(Q_{0}^{+} u, S_{0}\left(Q_{0}^{+} u\right)\right): u \in U\right\} .
$$

Demonstração: Vide Teorema 2.36 .

Fazemos aqui uma hipótese de estabilidade com respeito a linearização da equação (2.12).

(ES) Se $u_{\varepsilon}^{*} \in \mathscr{E}_{\varepsilon}$ e $u_{0}^{*} \in \mathscr{E}_{0}$ são tais que $u_{\varepsilon}^{*} \stackrel{E}{\longrightarrow} u_{0}^{*}$ então a família $\left\{A_{\varepsilon}^{-1} F_{\varepsilon}^{\prime}\left(u_{\varepsilon}^{*}\right)\right\}_{\varepsilon \in(0,1]}$ converge compactamente para o operador $A_{0}^{-1} F_{0}^{\prime}\left(u_{0}^{*}\right)$ e

$$
\frac{\left\|w_{\varepsilon}\left(u_{\varepsilon}^{*}, v\right)\right\|_{X_{\varepsilon}}}{\|v\|_{X_{\varepsilon}}}=o(1), \text { quando }\|v\|_{X_{\varepsilon}} \rightarrow 0
$$

uniformemente em $\varepsilon$.

Nas hipóteses da Seção 2.1.1 e supondo (ES) segue a semicontinuidade inferior da família de conjuntos de equilíbrios $\mathscr{E}_{\varepsilon}$.

Teorema 2.29. Se $u_{0}^{*} \in \mathscr{E}_{0}$ é um ponto de equilíbrio tal que $0 \notin \sigma\left(A_{0}-F_{0}^{\prime}\left(u_{0}^{*}\right)\right)$, então existe um $\boldsymbol{\delta}>$ 0 tal que existe um único $u_{\mathcal{\varepsilon}}^{*} \in \mathscr{E}_{\varepsilon} \cap B\left(E_{\varepsilon} u_{0}^{*}, \delta\right)$. Além disso, se $u_{0}^{*} \in \mathscr{E}_{0}$ é tal que $0 \notin \rho\left(A_{0}+F_{0}^{\prime}\left(u_{0}^{*}\right)\right)$, então da Proposição 2.24 sabemos que existem no máximo um número finito deles, digamos $u_{1}^{*}, \cdot, u_{r}^{*}$. Neste caso, existe um $\varepsilon_{0}$ tal que $\mathscr{E}_{\varepsilon}$ possui exatamente $r$ elementos $u_{\varepsilon, 1}^{*}, \cdot, u_{\varepsilon, r}^{*}$, para todo $\varepsilon \leqslant \varepsilon_{0}$ e ainda $u_{\varepsilon, i}^{*} \stackrel{E}{\longrightarrow} u_{i}^{*}$.

Além disso, se $u_{0}^{*}$ é hiperbólico então $u_{\varepsilon}^{*}$ também o é.

Demonstração: Observemos que $u_{\varepsilon}^{*} \in \mathscr{E}_{\varepsilon}$ se e somente é ponto fixo do operador compacto $-A_{\varepsilon}^{-1} F_{\varepsilon}(\cdot): X_{\varepsilon} \rightarrow X_{\varepsilon}$. Também, $0 \notin \sigma\left(A_{0}+F_{\varepsilon}^{\prime}\left(u_{\varepsilon}^{*}\right)\right)$ se e somente se $1 \notin \sigma\left(I-A_{\varepsilon}^{-1} F_{\varepsilon}^{\prime}\left(u_{\varepsilon}^{*}\right)\right)$. E segue que existe uma constante $\eta>0$ (independente de $\varepsilon$ ) tal que para qualquer $\varepsilon \leqslant \varepsilon_{0}, 0 \notin$ $\sigma\left(A_{0}+F_{\varepsilon}^{\prime}\left(u_{\varepsilon}^{*}\right)\right),\left\|v_{\varepsilon}+A_{\varepsilon}^{-1} F_{\varepsilon}\left(u_{\varepsilon}^{*}\right) v_{\varepsilon}\right\|_{X_{\varepsilon}} \geqslant 2 \eta\left\|v_{\varepsilon}\right\|_{X_{\varepsilon}}$. Se escrevermos

$$
A_{\varepsilon}^{-1} F_{\varepsilon}\left(u_{\varepsilon}^{*}+v_{\varepsilon}\right)-A_{\varepsilon}^{-1} F_{\varepsilon}\left(u_{\varepsilon}^{*}\right)-A_{\varepsilon}^{-1} F_{\varepsilon}^{\prime}\left(u_{\varepsilon}^{*}\right) v_{\varepsilon}=w_{\varepsilon}\left(u_{\varepsilon}^{*}, v_{\varepsilon}\right), \quad \frac{\left\|w_{\varepsilon}\left(u_{\varepsilon}^{*}, v_{\varepsilon}\right)\right\|_{X_{\varepsilon}}}{\left\|v_{\varepsilon}\right\|_{X_{\varepsilon}}} \leqslant h\left(\left\|v_{\varepsilon}\right\|_{X_{\varepsilon}}\right),
$$

onde $h:[0, \infty) \rightarrow \mathbb{R}$ pode ser tomada contínua com $h(0)=0$. Logo existe $\delta>0$ (independente de $\varepsilon)$ tal que

$$
\left\|w_{\varepsilon}\left(u_{\varepsilon}^{*}, v_{\varepsilon}\right)\right\|_{X_{\varepsilon}} \leqslant \eta\left\|v_{\varepsilon}\right\|_{X_{\varepsilon}}
$$


para $\left\|v_{\varepsilon}\right\|_{X_{\varepsilon}} \leqslant 2 \delta$.

Então, para $\left\|u_{\varepsilon}^{*}-u_{\varepsilon}\right\|_{X_{\varepsilon}} \leqslant 2 \delta$

$$
\left\|u_{\varepsilon}-A_{\varepsilon}^{-1} F_{\varepsilon}\left(u_{\varepsilon}\right)\right\|_{X_{\varepsilon}} \geqslant\left\|u_{\varepsilon}-u_{\varepsilon}^{*}-A_{\varepsilon}^{-1} F_{\varepsilon}^{\prime}\left(u_{\varepsilon}^{*}\right)\left(u_{\varepsilon}^{*}-u_{\varepsilon}\right)\right\|_{X_{\varepsilon}}-\left\|w_{\varepsilon}\left(u_{\varepsilon}^{*}, u_{\varepsilon}-u_{\varepsilon}^{*}\right)\right\|_{X_{\varepsilon}} \geqslant \eta\left\|u_{\varepsilon}-u_{\varepsilon}^{*}\right\| .
$$

Portanto $u_{\varepsilon}^{*}$ é um ponto de equilíbrio isolado. Isto e o fato que $u_{\varepsilon}^{*} \stackrel{E}{\longrightarrow} u_{0}^{*}$ demonstram o resultado.

Observação 2.30. Como $A_{\varepsilon}$ é setorial, tem resolvente compacto e $F_{\varepsilon}^{\prime}\left(u_{\varepsilon}^{*}\right) \in \mathscr{L}\left(X_{\varepsilon}^{\frac{1}{2}}, X_{\varepsilon}\right)$, então o operador $\bar{A}_{\varepsilon}$ é também setorial com resolvente compacto, valendo estimativas setoriais do resolvente uniformes em $\boldsymbol{\varepsilon}$. Logo com nossas hipóteses a família de operadores $\left\{\bar{A}_{\varepsilon}^{-1}\right\}_{\varepsilon \in(0,1]}$ converge compactamente para o operador $\bar{A}_{0}^{-1}$.

De fato, basta observar que $\left(A_{\varepsilon}-F_{\varepsilon}^{\prime}\left(u_{\varepsilon}^{*}\right)\right)^{-1}=\left(I-A_{\varepsilon}^{-1} F_{\varepsilon}^{\prime}\left(u_{\varepsilon}^{*}\right)\right)^{-1} A_{\varepsilon}^{-1}$

Com isso também para os operadores $\overline{A_{\varepsilon}}$ temos um análogo do Lema 2.10.

Lema 2.31. Seja $K$ um subconjunto compacto de $\rho\left(-\bar{A}_{0}\right)$. Então existe uma constante $\varepsilon_{K}>0$ de tal forma que $K \subset \rho\left(-\overline{A_{\varepsilon}}\right)$ para todo $\varepsilon \in\left(0, \varepsilon_{K}\right]$

$$
\sup _{\alpha \in[0,1]} \sup _{\varepsilon \in\left(0, \varepsilon_{K}\right]} \sup _{\mu \in K}\left\|\left(\mu+\overline{A_{\varepsilon}}\right)^{-1}\right\|_{\mathscr{L}\left(X_{\varepsilon}, X_{\varepsilon}^{\alpha}\right)}<\infty .
$$

Adicionalmente para $u_{0} \in X_{0}^{\frac{1}{2}}$

$$
\lim _{\varepsilon \rightarrow 0} \sup _{\mu \in K}\left\|\left(\mu+\overline{A_{\varepsilon}}\right)^{-1} u_{0}-\left(\mu+\overline{A_{0}}\right)^{-1} u_{0}\right\|_{X_{0}^{\frac{1}{2}}}=0 .
$$

Lembremos que os operadores $\bar{A}_{\varepsilon}$ possuem resolvente compacto, e, dessa forma, o espectro dos operadores $-\overline{A_{\mathcal{E}}}, \varepsilon \in\left[0, \varepsilon_{0}\right]$ consistem apenas de autovalores isolados, logo, de maneira inteiramente análoga à seção anterior, para cada $\mu \in \sigma\left(-\bar{A}_{0}\right)$, associamos seu auto-espaço generalizado $W\left(\lambda,-\bar{A}_{0}\right)=Q_{0}\left(\lambda,-\bar{A}_{0}\right) X_{0}$ onde

$$
Q_{0}\left(\lambda,-\bar{A}_{0}\right)=\frac{1}{2 \pi i} \int_{S_{\lambda, \delta}}\left(\xi I+\bar{A}_{0}\right)^{-1} d \xi
$$

com $\delta$ suficientemente pequeno de modo a $\sigma(A) \backslash\{\lambda\}$ não interceptar o disco $O_{\lambda, \delta}$.

Em particular o análogo do Lema 2.8 nos permite definir para $\varepsilon$ suficientemente pequeno os auto espaços generalizados $W\left(\lambda,-\bar{A}_{\varepsilon}\right)=Q\left(\lambda,-A_{\varepsilon}\right) X_{\varepsilon}$ onde

$$
Q\left(\lambda,-\bar{A}_{\varepsilon}\right)=\frac{1}{2 \pi i} \int_{S_{\lambda, \delta}}\left(\xi I+\bar{A}_{\varepsilon}\right)^{-1} d \xi .
$$

Portanto temos também as seguintes versões do Lema 2.12 e do Teorema 3.19. 
Lema 2.32. Sejam $\lambda \in \sigma\left(-\bar{A}_{0}\right)$ e $\delta$ suficientemente pequeno de modo a $\sigma\left(-\bar{A}_{0}\right) \backslash\{\lambda\}$ não interceptar o disco $O_{\lambda, \delta}$. Então $Q_{\varepsilon}\left(\lambda,-\bar{A}_{\mathcal{\varepsilon}}\right) \stackrel{C C}{\longrightarrow} Q_{0}\left(\lambda,-\bar{A}_{0}\right)$.

Teorema 2.33. Com as hipóteses do lema anterior as seguintes afirmações são válidas:

(i) para todo $\mu_{0} \in \sigma\left(-\bar{A}_{0}\right)$, existem seqüências $\varepsilon_{n} \rightarrow 0$ e $\left\{\mu_{\varepsilon_{n}}\right\}, \mu_{\varepsilon_{n}} \in \sigma\left(-\bar{A}_{\varepsilon_{n}}\right), n \in \mathbb{N}$, tais que $\mu_{\varepsilon_{n}} \rightarrow \mu_{0}$ quando $n \rightarrow \infty$.

(ii) se para alguma seqüência $\varepsilon_{n} \rightarrow 0$, e alguma seqüência $\left\{\mu_{\varepsilon_{n}}\right\}, \mu_{\varepsilon_{n}} \in \sigma\left(-\bar{A}_{\mathcal{E}_{n}}\right), n \in \mathbb{N}$, temos $\mu_{\varepsilon_{n}} \rightarrow \mu_{0}$ quando $n \rightarrow \infty$, então $\mu_{0} \in \sigma\left(-\bar{A}_{0}\right)$.

(iii) existe $\bar{\varepsilon}>0$ tal que $\operatorname{dim} W\left(\mu,-\bar{A}_{\varepsilon}\right)=\operatorname{dim} W\left(\mu,-\bar{A}_{0}\right)$, para todo $0<\varepsilon \leqslant \bar{\varepsilon}$.

(iv) para todo $u \in W\left(\mu_{0},-\bar{A}_{0}\right)$, existe uma seqüência $\left\{u_{\varepsilon}\right\}, u_{\varepsilon} \in W\left(\mu_{0},-\bar{A}_{\varepsilon}\right)$, tal que $u_{\varepsilon} \stackrel{\varepsilon \rightarrow 0}{\longrightarrow} u$.

(v) toda seqüência $\varepsilon_{n} \rightarrow 0,\left\{u_{\mathcal{E}_{n}}\right\}, u_{n} \in W\left(\mu,-\bar{A}_{\mathcal{E}_{n}}\right), n \in \mathbb{N}$, com $\left\|u_{\mathcal{E}_{n}}\right\|_{\mathcal{E}_{\varepsilon_{n}}}=1$ tem uma subseqüência convergente e todo ponto limite desta seqüência está em $W\left(\mu_{0},-\bar{A}_{0}\right)$.

Recordemos a definição da projeção $Q_{0}^{+}$dada por

$$
Q_{0}^{+}\left(\sigma_{0}^{+}\right)=\frac{1}{2 \pi i} \int_{\Gamma^{+}}\left(\mu+\bar{A}_{0}\right)^{-1} d \mu
$$

onde $\Gamma^{+}$é uma curva em $\left\{\mu \in \rho\left(-A_{0}\right): \operatorname{Re} \mu>0\right\}$, que envolve $\sigma_{0}^{+}$. Do Lema 2.31, segue a existência de um $\varepsilon_{\Gamma}$ tal que $\Gamma^{+} \subset \rho\left(-\bar{A}_{\varepsilon}\right)$ for $\varepsilon \in\left(0, \varepsilon_{\Gamma}^{+}\right]$. Seja $Q_{\varepsilon}^{+}\left(\sigma_{\varepsilon}^{+},-\bar{A}_{\varepsilon}\right)$ projeção dada por

$$
Q_{\varepsilon}^{+}\left(\sigma_{\varepsilon}^{+}\right)=\frac{1}{2 \pi i} \int_{\Gamma^{+}}\left(\mu+\bar{A}_{\varepsilon}\right)^{-1} d \mu .
$$

Consideremos $-\bar{A}_{\varepsilon}^{+}$e $-\bar{A}_{\varepsilon}^{-}$as restrições de $-\bar{A}_{\varepsilon}$ a $W_{\varepsilon}^{+}=Q_{\varepsilon}^{+}\left(\sigma_{\varepsilon}^{+}\right) X$ e ao espaço $W_{\varepsilon}^{-}=\left(I-Q_{\varepsilon}^{+}\left(\sigma_{\varepsilon}^{+}\right)\right) X$, respectivamente.

Teorema 2.34. São válidas as seguintes afirmações:

(i) existe um $\varepsilon_{0}>0$ tal que $\operatorname{dim} W\left(\sigma_{\varepsilon}^{+},-\bar{A}_{\varepsilon}^{+}\right)=\operatorname{dim} W\left({\sigma_{0}^{+}}^{+},-{\overline{A_{0}}}^{+}\right)$, para todo $0<\varepsilon \leqslant \varepsilon_{0}$.

(ii) para todo $u \in W\left({\sigma_{0}^{+}}^{+},{\overline{A_{0}}}^{+}\right)$, existe uma seqüência $\left\{u_{\varepsilon}\right\}, u^{\varepsilon} \in W\left(\sigma_{\varepsilon}^{+},-\overline{A_{\varepsilon}}\right)$, tal que $u^{\varepsilon} \stackrel{\varepsilon \rightarrow 0}{\longrightarrow} u$.

(iii) toda seqüência $\left\{u_{n}\right\}, u_{n} \in W\left(\sigma_{\varepsilon}^{+},-\bar{A}_{\varepsilon_{n}}\right), n \in \mathbb{N},, \varepsilon_{n} \rightarrow 0$, com $\left\|u_{n}\right\|_{X_{\varepsilon_{n}}}=1$ possui uma subseqüência convergente e todo ponto limite desta seqüência está em $W\left(\sigma_{0}^{+},-\overline{A_{0}^{+}}\right)$.

(iv) existem $\beta>0$ e $\varepsilon_{\beta}>0$ tais que $\sigma\left(-\bar{A}_{\varepsilon}^{+}\right) \cap\{\mu \in \mathbb{C}:|\operatorname{Re} \mu|>\beta\}=\varnothing, \varepsilon \in\left(0, \varepsilon_{\beta}\right]$. 
(v) existem $\frac{\pi}{2}>\phi_{\beta}>0, \omega_{\beta}>0$ e $\varepsilon_{\beta}>0$ tais que $\Sigma_{-\omega_{\beta}, \phi_{\beta}} \subset \rho\left(-\bar{A}_{\bar{\varepsilon}}^{-}\right), \varepsilon \in\left[0, \varepsilon_{\beta}\right]$ e

$$
\left\|\left(\mu+\bar{A}_{\varepsilon}^{-}\right)^{-1}\right\|_{\mathscr{L}\left(X_{\varepsilon}\right)} \leqslant \frac{M_{\beta}}{\left|\mu-\omega_{\beta}\right|} \text {, para todo } \mu \in \Sigma_{-\omega_{\beta}, \phi_{\beta}} \text { e para todo } \varepsilon \in\left[0, \varepsilon_{\beta}\right] .
$$

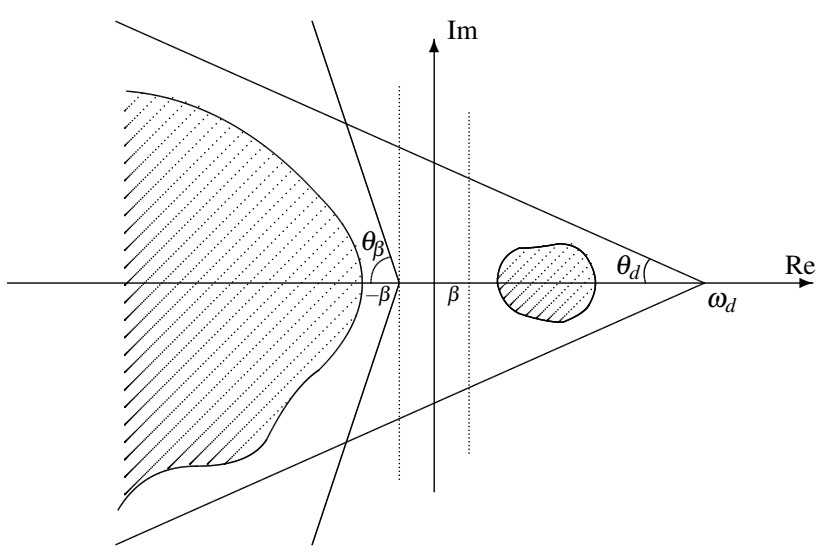

Figura 1

\subsubsection{Continuidade das variedades instáveis}

A existência de variedades invariantes é um tópico amplamente estudado, e pode ser encontrado com certo detalhe em [34]. Entretanto a seguinte prova é uma adaptação para englobar a possibilidade dos espaços envolvidos variarem com relação a um parâmetro.

Como estamos assumindo que todos os equilíbrios $u_{0}^{*} \in \mathscr{E}_{0}$ do problema (2.12) são hiperbólicos, sabemos que o conjunto $\sigma_{0}^{+}$consiste apenas de um número finito de autovalores com multiplicidade finita e para $\varepsilon$ suficientemente pequeno, o mesmo é também verdade para $\sigma_{\varepsilon}^{+}$.

Proposição 2.35. Dada uma seqüencia $\varepsilon_{n} \rightarrow 0$, seja $\left\{u_{\mathcal{\varepsilon}_{n}}^{*}\right\}, u_{\mathcal{E}_{n}}^{*} \in \mathscr{E}_{\varepsilon_{n}}$ tal que $u_{\mathcal{E}_{n}}^{*} \stackrel{E}{\longrightarrow} u_{0}^{*} \in \mathscr{E}_{0}$. Então existe um $\bar{\varepsilon}>0$ tal que todos os equilíbrios $u_{\mathcal{\varepsilon}}^{*} \in \mathscr{E}_{\varepsilon}$ são hiperbólicos para $0 \leqslant \varepsilon \leqslant \bar{\varepsilon}$, e $\left\|\bar{A}_{\mathcal{\varepsilon}_{n}}^{-1}\right\| \leqslant C$, com $C$ independente de $\varepsilon_{n}$. Além disso, $Q_{\varepsilon}^{+}$converge compactamente para $Q_{0}^{+}$(e então $\operatorname{posto}\left(\mathrm{Q}_{\varepsilon}^{+}\right)=\operatorname{posto}\left(\mathrm{Q}_{0}^{+}\right)$, para $\left.0<\varepsilon \leqslant \bar{\varepsilon}\right)$, e a família de conjuntos $\sigma_{\varepsilon}^{+}$é contínua em $\varepsilon=0$.

Podemos, portanto, concluir que para $\varepsilon \in[0, \bar{\varepsilon}]$ existem constantes (independentes de $\varepsilon$ ) $\beta>0$ e $M_{\beta} \geqslant 1$, tais que

$$
\begin{aligned}
& \left\|e^{-\bar{A}_{\varepsilon} t} Q_{\mathcal{\varepsilon}}^{+}\right\|_{\mathscr{L}\left(X_{\varepsilon}\right)} \leqslant M_{\beta} e^{\beta t}, \quad t \leqslant 0 \\
& \left\|e^{-\bar{A}_{\varepsilon} t}\left(I-Q_{\varepsilon}^{+}\right)\right\|_{\mathscr{L}\left(X_{\varepsilon}, X_{\varepsilon}^{\frac{1}{2}}\right)} \leqslant M_{\beta} t^{-\frac{1}{2}} e^{-\beta t}, \quad t>0 .
\end{aligned}
$$


Para mostrarmos que as variedades instáveis dos pontos de equilíbrio estão, num certo sentido, próximas quando $\varepsilon$ é suficientemente pequeno, fazemos uso da dicotomia acima e também da convergência da parte linear obtida nas seções precedentes.

Ao decompormos o espaço $X_{\varepsilon}^{\frac{1}{2}}=Q_{\varepsilon}^{+} X_{\varepsilon}^{\frac{1}{2}} \oplus\left(I-Q_{\varepsilon}^{+}\right) X_{\varepsilon}^{\frac{1}{2}}$, induzimos também uma decomposição, $v_{\varepsilon}=w_{\varepsilon}+z_{\varepsilon}$ da solução de $(2.12)$, com $w_{\varepsilon}=Q_{\varepsilon}^{+} v_{\varepsilon}$ e $z_{\varepsilon}=\left(I-Q_{\varepsilon}^{+}\right) v_{\varepsilon}$. Ainda, $w_{\varepsilon}$ e $z_{\varepsilon}$ satisfazem

$$
\begin{aligned}
& \dot{w}_{\varepsilon}+\bar{A}_{\varepsilon}^{+} w_{\varepsilon}=H_{\varepsilon}\left(w_{\varepsilon}, z_{\varepsilon}\right):=\quad Q_{\varepsilon}^{+}\left[F_{\varepsilon}\left(w_{\varepsilon}+z_{\varepsilon}+u_{\varepsilon}^{*}\right)-F_{\varepsilon}\left(u_{\varepsilon}^{*}\right)-F_{\varepsilon}^{\prime}\left(u_{\varepsilon}^{*}\right)\left(w_{\varepsilon}+z_{\varepsilon}\right)\right] \\
& \dot{z}_{\varepsilon}+\bar{A}_{\varepsilon}^{-} z_{\varepsilon}=G_{\varepsilon}\left(w_{\varepsilon}, z_{\varepsilon}\right):=\left(I-Q_{\varepsilon}^{+}\right)\left[F_{\varepsilon}\left(w_{\varepsilon}+z_{\varepsilon}+u_{\varepsilon}^{*}\right)-F_{\varepsilon}\left(u_{\varepsilon}^{*}\right)-F_{\varepsilon}^{\prime}\left(u_{\varepsilon}^{*}\right)\left(w_{\varepsilon}+z_{\varepsilon}\right)\right]
\end{aligned}
$$

onde $\bar{A}_{\varepsilon}^{+}$(resp. $\left.\bar{A}_{\varepsilon}^{-}\right)$é a restrição do operador $\bar{A}_{\varepsilon}$ a imagem de $Q_{\varepsilon}^{+}$(resp. núcleo de $Q_{\varepsilon}^{+}$).

Observemos que $H_{\varepsilon}(0,0)=0=G_{\varepsilon}(0,0)$. Além disso, as hipóteses sobre $F_{\varepsilon}$ garantem que as aplicações $H_{\varepsilon}$ e $G_{\varepsilon}$ sejam continuamente Fréchet diferenciáveis com $H_{\varepsilon}^{\prime}(0,0) \equiv 0 \equiv G_{\varepsilon}^{\prime}(0,0)$.

Agora, da limitação uniforme das projeções $Q_{\varepsilon}^{+}, \varepsilon \in\left[0, \varepsilon_{0}\right]$, e da observação do parágrafo anterior, dado $\rho>0$, existem $\bar{\varepsilon}>0$ e $\delta>0$ tal que se $\|w\|_{X_{\varepsilon}^{\frac{1}{2}}}+\|z\|_{X_{\varepsilon}^{\frac{1}{2}}}<\delta$ e $\varepsilon \leqslant \bar{\varepsilon}$, então

$$
\begin{aligned}
& \left\|H_{\varepsilon}(w, z)\right\|_{X_{\varepsilon}^{\frac{1}{2}}} \leqslant \rho, \quad\left\|G_{\varepsilon}(w, z)\right\|_{X_{\varepsilon}} \leqslant \rho, \\
& \left\|H_{\varepsilon}(w, z)-H_{\varepsilon}(\tilde{w}, \tilde{z})\right\|_{X_{\varepsilon}^{\frac{1}{2}}} \leqslant \rho\left(\|w-\tilde{w}\|_{X_{\varepsilon}^{\frac{1}{2}}}+\|z-\tilde{z}\|_{X_{\varepsilon}^{\frac{1}{2}}}\right), \\
& \left\|G_{\varepsilon}(w, z)-G_{\varepsilon}(\tilde{w}, \tilde{z})\right\|_{X_{\varepsilon}} \leqslant \rho\left(\|w-\tilde{w}\|_{X_{\varepsilon}^{\frac{1}{2}}}+\|z-\tilde{z}\|_{X_{\varepsilon}^{\frac{1}{2}}}\right) .
\end{aligned}
$$

O fato de podermos escolher $\rho$ e $\delta$ uniformes para $\varepsilon \in[0, \bar{\varepsilon}]$ satisfazendo as desigualdades acima é o ponto chave para obtermos que as variedades instáveis locais estão definidas em uma pequena vizinhança do ponto de equilíbrio $u_{\varepsilon}^{*}$, uniformemente para $\varepsilon \leqslant \bar{\varepsilon}$. Nosso interesse é no comportamento das soluções próximas a $(0,0)$, assim podemos cortar as não-linearidades $H_{\varepsilon}$ e $G_{\varepsilon}$ fora da bola $B_{\delta}(0,0)$ de modo que as estimativas $(2.29)$ permaneçam válidas para todo $(w, z) \in X_{\varepsilon}^{\frac{1}{2}}$.

A seguir mostraremos que para $\rho>0$ escolhido apropriadamente, a variedade instável de $u_{\mathcal{E}}^{*} \in \mathscr{E}_{\mathcal{E}}$ é dada localmente como o gráfico

$$
\mathrm{W}_{\varepsilon}^{u}=\left\{(w, z) \in X_{\mathcal{\varepsilon}}^{\frac{1}{2}}: z=S_{\varepsilon}^{*}(w), w \in Q_{\varepsilon}^{+} X_{\varepsilon}^{\frac{1}{2}}\right\},
$$

de uma aplicação $S_{\varepsilon}^{*}: Q_{\varepsilon}^{+} X_{\varepsilon}^{\frac{1}{2}} \rightarrow\left(I-Q_{\varepsilon}^{+}\right) X_{\varepsilon}^{\frac{1}{2}}$ limitada e Lipschitz contínua. Além disso, para qualquer $R>0$, mostraremos que

$$
\sup _{\substack{\frac{1}{2} \\ w \in B_{\varepsilon}^{X^{2}}(0, R)}}\left\|S_{\varepsilon}^{*}(w)-E_{\varepsilon} S_{0}^{*}(w)\right\|_{X_{\varepsilon}^{\frac{1}{2}}} \stackrel{\varepsilon \rightarrow 0}{\longrightarrow} 0 .
$$

Para fixar notação, observemos que para constantes apropriadas $M, \beta$, (independentes de 
$\varepsilon \in[0, \bar{\varepsilon}])$, são válidas as seguintes estimativas

$$
\begin{aligned}
& \left\|e^{-\bar{A}_{\varepsilon}^{-} t} z\right\|_{X_{\varepsilon}^{\frac{1}{2}}} \leqslant M e^{-\beta t}\|z\|_{X_{\varepsilon}^{\frac{1}{2}}}, \quad t \geqslant 0, \\
& \left\|e^{-\bar{A}_{\varepsilon}^{-} t} z\right\|_{X_{\varepsilon}^{\frac{1}{2}}} \leqslant M t^{-\frac{1}{2}} e^{-\beta t}\|z\|_{X_{\varepsilon}}, \quad t>0, \\
& \left\|e^{-\bar{A}_{\varepsilon}^{+} t} v\right\|_{X_{\varepsilon}^{\frac{1}{2}}} \leqslant M e^{\beta t}\|v\|_{X_{\varepsilon}^{\frac{1}{2}}}, \quad t \leqslant 0 .
\end{aligned}
$$

Teorema 2.36. Seja $u_{0}^{*} \in \mathscr{E}_{0}$ um ponto de equilíbrio hiperbólico. Pelo Teorema 2.29 existem $\bar{\delta}>0$ e $\bar{\varepsilon}_{0}>0$ tais que existe um único $u_{\varepsilon}^{*} \in \mathscr{E}_{\varepsilon}$ com $\left\|u_{\varepsilon}^{*}-u_{0}^{*}\right\|_{X_{\varepsilon}^{\frac{1}{2}}}<\bar{\delta}$, para todo $\varepsilon \in\left[0, \bar{\varepsilon}_{0}\right]$. Então existem $\delta$ e $\varepsilon_{0}>0$ tal que se $W_{\delta}^{u}\left(u_{\varepsilon}^{*}\right):=W^{u}\left(u_{\varepsilon}^{*}\right) \cap B\left(\delta, u_{\varepsilon}^{*}\right)$ entã

$$
\lim _{\varepsilon \rightarrow 0} \sup _{u_{\varepsilon} \in W_{\delta}^{u}\left(u_{\varepsilon}^{*}\right)} \inf _{u_{0} \in W_{\delta}^{u}\left(u_{0}^{*}\right)}\left\|u_{\varepsilon}-E_{\varepsilon} u_{0}\right\|_{X_{\varepsilon}^{\frac{1}{2}}}=0 .
$$

Demonstração: Mostremos primeiramente a existência da variedade instável local do equilíbrio $u_{\varepsilon}^{*}$ como um gráfico.

$$
\begin{aligned}
& \mathscr{X}_{\varepsilon}=\left\{S_{\varepsilon}: Q_{\varepsilon}^{+} X_{\varepsilon}^{\frac{1}{2}} \rightarrow\left(I-Q_{\varepsilon}^{+}\right) X_{\varepsilon}^{\frac{1}{2}}: \sup _{w \in X_{\varepsilon}^{\frac{1}{2}}}\left\|S_{\varepsilon} Q_{\varepsilon}^{+}(w)\right\|_{X_{\varepsilon}^{\frac{1}{2}}} \leqslant D,\left\|S_{\varepsilon} Q_{\varepsilon}^{+}(w)-S_{\varepsilon} Q_{\varepsilon}^{+}(\tilde{w})\right\|_{X_{\varepsilon}^{\frac{1}{2}}} \leqslant \Delta\|w-\tilde{w}\|_{X_{\varepsilon}^{\frac{1}{2}}},\right. \\
& \left.\quad w, \tilde{w} \in X_{\varepsilon}^{\frac{1}{2}}\right\},
\end{aligned}
$$

munido da norma $\left\|S_{\varepsilon}\right\|_{\mathscr{X}_{\varepsilon}}=\sup _{w \in X_{\varepsilon}^{\frac{1}{2}}}\left\|S_{\varepsilon} Q_{\varepsilon}^{+}(w)\right\|_{X_{\varepsilon}^{\frac{1}{2}}}$. Não é difícil ver que $\left(\mathscr{X}_{\varepsilon},\|\cdot\|_{\mathscr{X}_{\varepsilon}}\right)$ é um espaço métrico completo.

Seja $w_{\varepsilon}(t)=\psi\left(t, \tau, \eta, S_{\varepsilon}\right)$ a solução de

$$
\left\{\begin{array}{l}
\dot{w}_{\varepsilon}+\bar{A}_{\varepsilon}^{+} w_{\varepsilon}=H_{\varepsilon}\left(w_{\varepsilon}, S_{\varepsilon} Q_{\varepsilon}^{+}\left(w_{\varepsilon}\right)\right), \quad t<\tau \\
w_{\varepsilon}(\tau)=\eta
\end{array}\right.
$$

$\operatorname{com} S_{\varepsilon} \in \mathscr{X}_{\varepsilon}$ e $\eta \in Q_{\varepsilon}^{+} X_{\varepsilon}^{\frac{1}{2}}$. Definimos $\Phi: \mathscr{X}_{\varepsilon} \rightarrow \mathscr{X}_{\varepsilon}$ por

$$
\Phi\left(S_{\varepsilon}\right)(\eta)=\int_{-\infty}^{\tau} e^{-\bar{A}_{\varepsilon}^{-}(\tau-s)} G_{\varepsilon}\left(w_{\varepsilon}(s), S_{\varepsilon} Q_{\varepsilon}^{+}\left(w_{\varepsilon}(s)\right)\right) d s .
$$

Observemos que

$$
\left\|\Phi\left(S_{\varepsilon}\right)(\eta)\right\|_{X_{\varepsilon}^{\frac{1}{2}}} \leqslant \int_{-\infty}^{\tau} M(\tau-s)^{-\frac{1}{2}} e^{-\beta(\tau-s)} \rho d s=\rho M \beta^{\frac{-1}{2}} \Gamma\left(\frac{1}{2}\right) .
$$

Logo para $\rho$ suficientemente pequeno temos $\left\|\Phi\left(S_{\varepsilon}\right)\right\|_{\mathscr{X}_{\varepsilon}} \leqslant D$.

Suponhamos agora que $S_{\varepsilon}, \tilde{S}_{\varepsilon} \in \mathscr{X}_{\varepsilon}, \quad \eta, \tilde{\eta} \in Q_{\varepsilon}^{+} X_{\varepsilon}^{\frac{1}{2}}, \quad$ e $\operatorname{sejam} w_{\varepsilon}(t)=\psi\left(t, \tau, \eta, S_{\varepsilon}\right)$, $\tilde{w}_{\varepsilon}(t)=\psi\left(t, \tau, \tilde{\eta}, \tilde{S}_{\varepsilon}\right)$ Então

$$
w_{\varepsilon}(t)-\tilde{w}_{\varepsilon}(t)=e^{-\bar{A}_{\varepsilon}^{+}(t-\tau)}(\eta-\tilde{\eta})+\int_{\tau}^{t} e^{-\bar{A}_{\varepsilon}^{+}(t-s)}\left[H_{\varepsilon}\left(w_{\varepsilon}, S_{\varepsilon} Q_{\varepsilon}^{+}\left(w_{\varepsilon}\right)\right)-H_{\varepsilon}\left(\tilde{w}_{\varepsilon}, \tilde{S}_{\varepsilon} Q_{\varepsilon}^{+}\left(\tilde{w}_{\varepsilon}\right)\right)\right] d s .
$$


Logo

$$
\begin{aligned}
\left\|w_{\varepsilon}(t)-\tilde{w}_{\varepsilon}(t)\right\|_{X_{\varepsilon}^{\frac{1}{2}}} & \leqslant M e^{\beta(t-\tau)}\|\eta-\tilde{\eta}\|_{X_{\varepsilon}^{\frac{1}{2}}}+M \int_{t}^{\tau} e^{\beta(t-s)}\left\|H_{\varepsilon}\left(w_{\varepsilon}, S_{\varepsilon} Q_{\varepsilon}^{+}\left(w_{\varepsilon}\right)\right)-H_{\varepsilon}\left(\tilde{w}_{\varepsilon}, \tilde{S}_{\varepsilon} Q_{\varepsilon}^{+}\left(\tilde{w}_{\varepsilon}\right)\right)\right\|_{X_{\varepsilon}^{\frac{1}{2}}} d s \\
& \leqslant M e^{\beta(t-\tau)}\|\eta-\tilde{\eta}\|_{X_{\varepsilon}^{\frac{1}{2}}}+\rho M \int_{t}^{\tau} e^{\beta(t-s)}\left[\left\|S_{\varepsilon} Q_{\varepsilon}^{+}\left(w_{\varepsilon}\right)-\tilde{S}_{\varepsilon} Q_{\varepsilon}^{+}\left(\tilde{w}_{\varepsilon}\right)\right\|_{X_{\varepsilon}^{\frac{1}{2}}}+\left\|w_{\varepsilon}-\tilde{w}_{\varepsilon}\right\|_{X_{\varepsilon}^{\frac{1}{2}}}\right] d s \\
& \leqslant M e^{\beta(t-\tau)}\|\eta-\tilde{\eta}\|_{X_{\varepsilon}^{\frac{1}{2}}}+\rho M \int_{t}^{\tau} e^{\beta(t-s)}\left[(1+\Delta)\left\|w_{\varepsilon}-\tilde{w}_{\varepsilon}\right\|_{X_{\varepsilon}^{\frac{1}{2}}}+\left\|S_{\varepsilon}-\tilde{S}_{\varepsilon}\right\|_{\mathscr{X}_{\varepsilon}}\right] d s \\
& \leqslant M e^{\beta(t-\tau)}\|\eta-\tilde{\eta}\|_{X_{\varepsilon}^{\frac{1}{2}}}+\rho M(1+\Delta) \int_{t}^{\tau} e^{\beta(t-s)}\left\|w_{\varepsilon}-\tilde{w}_{\varepsilon}\right\|_{X_{\varepsilon}^{\frac{1}{2}}} d s+\rho M\left\|S_{\varepsilon}-\tilde{S}_{\varepsilon}\right\|_{\mathscr{X}_{\varepsilon}} \int_{t}^{\tau} e^{\beta(t-s)} d s .
\end{aligned}
$$

Seja $\phi(t)=e^{-\beta(t-\tau)}\left\|w_{\varepsilon}(t)-\tilde{w}_{\varepsilon}(t)\right\|_{X_{\varepsilon}^{\frac{1}{2}}}$ Então

$$
\phi(t) \leqslant M\|\eta-\tilde{\eta}\|_{X_{\varepsilon}^{\frac{1}{2}}}+\rho M\left\|S_{\varepsilon}-\tilde{S}_{\mathcal{E}}\right\|_{\mathscr{X}_{\varepsilon}} \int_{t}^{\tau} e^{\beta(\tau-s)} d s+\rho M(1+\Delta) \int_{t}^{\tau} \phi(s) d s .
$$

Pela Desigualdade de Gronwall Generalizada

$$
\begin{aligned}
\left\|w_{\varepsilon}(t)-\tilde{w}_{\varepsilon}(t)\right\|_{X_{\varepsilon}^{\frac{1}{2}}} & \leqslant\left(M e^{\beta(t-\tau)}\|\eta-\tilde{\eta}\|_{X_{\varepsilon}^{\frac{1}{2}}}+\rho M \int_{t}^{\tau} e^{\beta(t-s)} d s\left\|S_{\varepsilon}-\tilde{S}_{\varepsilon}\right\|_{\mathscr{X}_{\varepsilon}}\right) e^{\rho M(1+\Delta)(\tau-t)} \\
& \leqslant\left(M\|\eta-\tilde{\eta}\|_{X_{\varepsilon}^{\frac{1}{2}}}+\rho M \beta^{-1}\left\|S_{\varepsilon}-\tilde{S}_{\varepsilon}\right\|_{\mathscr{X}_{\varepsilon}}\right) e^{\rho M(1+\Delta)(\tau-t)}
\end{aligned}
$$

Por outro lado,

$$
\begin{aligned}
\left\|\Phi\left(S_{\varepsilon}\right)(\eta)-\Phi\left(\tilde{S}_{\varepsilon}\right)(\tilde{\eta})\right\|_{X_{\varepsilon}^{\frac{1}{2}}} & \leqslant M \int_{-\infty}^{\tau}(\tau-s)^{-\frac{1}{2}} e^{-\beta(\tau-s)}\left\|G_{\varepsilon}\left(w_{\varepsilon}, S_{\varepsilon} Q_{\varepsilon}^{+}\left(w_{\varepsilon}\right)\right)-G_{\varepsilon}\left(\tilde{w}_{\varepsilon}, \tilde{S}_{\varepsilon} Q_{\varepsilon}^{+}\left(\tilde{w}_{\varepsilon}\right)\right)\right\|_{X_{\varepsilon}} d s \\
& \leqslant \rho M \int_{-\infty}^{\tau}(\tau-s)^{-\frac{1}{2}} e^{-\beta(\tau-s)}\left((1+\Delta)\left\|w_{\varepsilon}(s)-\tilde{w}_{\varepsilon}(s)\right\|_{X_{\varepsilon}^{\frac{1}{2}}}+\left\|S_{\varepsilon}-\tilde{S}_{\varepsilon}\right\|_{\mathscr{X}_{\varepsilon}}\right) d s .
\end{aligned}
$$

Agora, das estimativas para $\left\|w_{\varepsilon}-\tilde{w}_{\varepsilon}\right\|_{X_{\varepsilon}^{\frac{1}{2}}}$, obtemos

$$
\begin{aligned}
\left\|\Phi\left(S_{\varepsilon}\right)(\eta)-\Phi\left(\tilde{S}_{\varepsilon}\right)(\tilde{\eta})\right\|_{X_{\varepsilon}^{\frac{1}{2}} \leqslant} & \rho M \beta^{-\frac{1}{2}} \Gamma\left(\frac{1}{2}\right)\left(1+\frac{\rho M(1+\Delta)}{\beta-\rho M(1+\Delta)}\right)\left\|S_{\varepsilon}-\tilde{S}_{\varepsilon}\right\|_{\mathscr{X}_{\varepsilon}} \\
& +2 \rho M^{2}(1+\Delta) \beta^{-\frac{1}{2}} \Gamma\left(\frac{1}{2}\right)\|\eta-\tilde{\eta}\|_{X_{\varepsilon}^{\frac{1}{2}}} .
\end{aligned}
$$

Tomando $\rho$ de modo a $\frac{\beta}{2} \leqslant \beta-\rho M(1-\Delta) \leqslant \beta$, sejam

$$
I_{S}(\varepsilon):=\rho M \beta^{-\frac{1}{2}} \Gamma\left(\frac{1}{2}\right)\left(1+\frac{\rho M(1+\Delta)}{\beta-\rho M(1+\Delta)}\right)
$$

$\mathrm{e}$

$$
I_{\eta}(\varepsilon):=2 \rho M^{2}(1+\Delta) \beta^{-\frac{1}{2}} \Gamma\left(\frac{1}{2}\right)
$$

Dado $\theta<1$, é fácil ver que existe um $\rho_{0}$ tal que, para $\rho \leqslant \rho_{0}, I_{S}(\varepsilon) \leqslant \theta$ e $I_{\eta}(\varepsilon) \leqslant \Delta$ e portanto

$$
\left\|\Phi\left(S_{\varepsilon}\right)(\eta)-\Phi\left(\tilde{S}_{\varepsilon}\right)(\tilde{\eta})\right\|_{X_{\varepsilon}^{\frac{1}{2}}} \leqslant \Delta\|\eta-\tilde{\eta}\|_{X_{\varepsilon}^{\frac{1}{2}}}+\theta\left\|S_{\varepsilon}-\tilde{S}_{\mathcal{\varepsilon}}\right\|_{\mathscr{X}_{\varepsilon}}
$$


As desigualdades (2.32) e (2.33), garantem que $\Phi$ é uma contração em $\mathscr{X}_{\varepsilon}$. Seja $S_{\varepsilon}^{*}=\Phi\left(S_{\varepsilon}^{*}\right) \in \mathscr{X}_{\varepsilon}$ o único ponto fixo de $\Phi$.

Provaremos agora que $\mathrm{W}_{\varepsilon}^{u}:=\left\{\left(w, S_{\varepsilon}^{*}(w)\right) \in X_{\varepsilon}^{\frac{1}{2}}: w \in Q_{\varepsilon}^{+} X_{\varepsilon}^{\frac{1}{2}}\right\}$ é uma variedade invariante para (2.28). Sejam $\left(w_{0}, z_{0}\right) \in \mathrm{W}_{\varepsilon}^{u}$, com $z_{0}=S_{\mathcal{\varepsilon}}^{*}\left(w_{0}\right)$ e $w_{\varepsilon}^{*}(t)$ a solução do problema de Cauchy

$$
\left\{\begin{array}{l}
\dot{w}_{\varepsilon}+\bar{A}_{\varepsilon}^{+} w_{\varepsilon}=H_{\varepsilon}\left(w_{\varepsilon}, S_{\varepsilon}^{*}\left(w_{\varepsilon}\right)\right) \\
w_{\varepsilon}(0)=w_{0}
\end{array}\right.
$$

Isto define uma curva $\left(w_{\mathcal{\varepsilon}}^{*}(t), S_{\mathcal{\varepsilon}}^{*}\left(w_{\mathcal{\varepsilon}}^{*}(t)\right)\right) \in \mathrm{W}_{\varepsilon}^{u}, t \in \mathbb{R}$. Observemos ainda que a única solução de $\dot{z}_{\varepsilon}+\bar{A}_{\varepsilon}^{-} z_{\varepsilon}=G_{\varepsilon}\left(w_{\varepsilon}^{*}, S_{\mathcal{\varepsilon}}^{*}\left(w_{\varepsilon}^{*}\right)\right)$ que permanece limitada quando $t \rightarrow-\infty$ é

$$
z_{\mathcal{\varepsilon}}^{*}(t)=\int_{-\infty}^{t} e^{-\bar{A}_{\varepsilon}^{-}(t-r)} G_{\mathcal{\varepsilon}}\left(w_{\varepsilon}^{*}(s), S_{\mathcal{\varepsilon}}^{*}\left(w_{\varepsilon}^{*}(s)\right) d s=S_{\mathcal{\varepsilon}}^{*}\left(w_{\mathcal{\varepsilon}}^{*}(t)\right) .\right.
$$

Portanto, $\left(w_{\varepsilon}^{*}(t), S_{\mathcal{\varepsilon}}^{*}\left(w_{\varepsilon}^{*}(t)\right)\right)$ é uma solução do sistema (2.28) passando por $\left(w_{0}, z_{0}\right)$, mostrando assim a invariância de $\mathrm{W}_{\varepsilon}^{u}$.

A seguir provaremos que se $H_{\varepsilon}$ e $G_{\varepsilon}$ satisfazem $(2.29)$ para todo $\left(w_{\varepsilon}, z_{\varepsilon}\right) \in X_{\varepsilon}^{\frac{1}{2}} \oplus\left(I-Q_{\varepsilon}^{+}\right) X_{\varepsilon}^{\frac{1}{2}}$ $\operatorname{com} \rho$ suficientemente pequeno e se $\left(w_{\varepsilon}(t), z_{\varepsilon}(t)\right), t \in \mathbb{R}$, é uma solução global de (2.28) que está em $W_{\varepsilon}^{u}$, então $z_{\varepsilon}(t)=S_{\varepsilon}^{*}\left(w_{\varepsilon}(t)\right)$, para todo $t \in \mathbb{R}$. Com este intuito mostraremos que existem constantes $M \geqslant 1$ e $\gamma>0$ tais que

$$
\left\|z_{\varepsilon}(t)-S_{\mathcal{\varepsilon}}^{*}\left(w_{\varepsilon}(t)\right)\right\|_{X_{\varepsilon}} \leqslant M e^{-\gamma\left(t-t_{0}\right)}\left\|z_{\varepsilon}\left(t_{0}\right)-S_{\varepsilon}^{*}\left(w_{\varepsilon}\left(t_{0}\right)\right)\right\|_{X_{\varepsilon}}, \quad t_{0} \leqslant t
$$

Fazendo $t_{0} \rightarrow-\infty$, obteremos $z_{\varepsilon}(t)=S_{\mathcal{\varepsilon}}^{*}\left(w_{\varepsilon}(t)\right)$ para todo $t \in \mathbb{R}$.

De fato, seja $y_{\varepsilon}(s, t), s \leqslant t$, a solução de

$$
\left\{\begin{array}{l}
\dot{y}_{\varepsilon}+\bar{A}_{\varepsilon}^{+} y_{\varepsilon}=H_{\varepsilon}\left(y_{\varepsilon}, S_{\varepsilon}^{*}\left(y_{\varepsilon}\right)\right), \quad s \leqslant t \\
y_{\varepsilon}(t, t)=w_{\varepsilon}(t)
\end{array}\right.
$$

Colocando $\xi_{\varepsilon}(t)=z_{\varepsilon}(t)-S_{\varepsilon}^{*}\left(w_{\varepsilon}(t)\right)$, temos

$$
\begin{aligned}
\left\|y_{\varepsilon}(s, t)-w_{\varepsilon}(s)\right\|_{X_{\varepsilon}^{\frac{1}{2}}} & =\left\|\int_{t}^{s} e^{-\bar{A}_{\varepsilon}^{+}(s-\theta)}\left[H_{\varepsilon}\left(y_{\varepsilon}(\theta, t), S_{\varepsilon}^{*}\left(y_{\varepsilon}(\theta, t)\right)\right)-H_{\varepsilon}\left(w_{\varepsilon}(\theta), z_{\varepsilon}(\theta)\right)\right] d \theta\right\|_{X_{\varepsilon}^{\frac{1}{2}}} \\
& \leqslant \rho M \int_{s}^{t} e^{\beta(s-\theta)}\left[(1+\Delta)\left\|y_{\varepsilon}(\theta, t)-w_{\varepsilon}(\theta)\right\|_{X_{\varepsilon}^{\frac{1}{2}}}+\left\|S_{\varepsilon}^{*}\left(w_{\varepsilon}(\theta)\right)-z_{\varepsilon}(\theta)\right\|_{X_{\varepsilon}^{\frac{1}{2}}}\right] d \theta \\
& \leqslant \rho M \int_{s}^{t} e^{\beta(s-\theta)}\left[(1+\Delta)\left\|y_{\varepsilon}(\theta, t)-w_{\varepsilon}(\theta)\right\|_{X_{\varepsilon}^{\frac{1}{2}}}+\left\|\xi_{\varepsilon}(\theta)\right\|_{X_{\varepsilon}^{\frac{1}{2}}}\right] d \theta .
\end{aligned}
$$

Se $\phi_{\varepsilon}(s)=e^{-\beta s}\left\|y_{\varepsilon}(s, t)-w_{\varepsilon}(s)\right\|_{X_{\varepsilon}^{\frac{1}{2}}}$, então

$$
\phi_{\varepsilon}(s) \leqslant \rho M(1+\Delta) \int_{s}^{t} \phi_{\varepsilon}(\theta) d \theta+\rho M \int_{s}^{t} e^{-\beta \theta}\left\|\xi_{\varepsilon}(\theta)\right\|_{X_{\varepsilon}^{\frac{1}{2}}} d \theta, \quad s \leqslant t .
$$

Pela Desigualdade de Gronwall temos

$$
\left\|y_{\varepsilon}(s, t)-w_{\mathcal{E}}(s)\right\|_{X_{\varepsilon}^{\frac{1}{2}}} \leqslant \rho M \int_{s}^{t} e^{-(\beta-\rho M(1+\Delta))(\theta-s)}\left\|\xi_{\varepsilon}(\theta)\right\|_{X_{\varepsilon}^{\frac{1}{2}}} d \theta, \quad s \leqslant t .
$$


Seja agora $s \leqslant t_{0} \leqslant t$. Então,

$$
\begin{aligned}
\left\|y_{\mathcal{\varepsilon}}(s, t)-y_{\varepsilon}\left(s, t_{0}\right)\right\|_{X_{\varepsilon}^{\frac{1}{2}}} & =\left\|e^{-\bar{A}_{\varepsilon}^{+}\left(s-t_{0}\right)}\left[y_{\varepsilon}\left(t_{0}, t\right)-w\left(t_{0}\right)\right]\right\|_{X_{\varepsilon}^{\frac{1}{2}}} \\
& +\| \int_{t_{0}}^{s} e^{-\bar{A}_{\varepsilon}^{+}(s-\theta)}\left[H_{\varepsilon}\left(y_{\varepsilon}(\theta, t), S_{\varepsilon}^{*}\left(y_{\varepsilon}(\theta, t)\right)-H_{\varepsilon}\left(y_{\varepsilon}\left(\theta, t_{0}\right), S_{\varepsilon}^{*}\left(y_{\varepsilon}\left(\theta, t_{0}\right)\right)\right)\right] d \theta \|_{X_{\varepsilon}^{\frac{1}{2}}}\right. \\
& \leqslant \rho M^{2} e^{\beta\left(s-t_{0}\right)} \int_{t_{0}}^{t} e^{-(\beta-\rho M(1+\Delta))\left(\theta-t_{0}\right)}\left\|\xi^{\varepsilon}(\theta)\right\|_{X_{\varepsilon}^{\frac{1}{2}}} d \theta \\
& +\rho M \int_{s}^{t_{0}} e^{\beta(s-\theta)}(1+\Delta)\left\|y_{\varepsilon}(\theta, t)-y_{\varepsilon}\left(\theta, t_{0}\right)\right\|_{X_{\varepsilon}^{\frac{1}{2}}} d \theta
\end{aligned}
$$

Novamente pela Desigualdade de Gronwall,

$$
\left\|y_{\varepsilon}(s, t)-y_{\varepsilon}\left(s, t_{0}\right)\right\|_{X_{\varepsilon}^{\frac{1}{2}}} \leqslant \rho M^{2} \int_{t_{0}}^{t} e^{-(\beta-\rho M(1+\Delta))(\theta-s)}\left\|\xi_{\varepsilon}(\theta)\right\|_{X_{\varepsilon}^{\frac{1}{2}}} d \theta
$$

Vamos estimar agora $\xi_{\varepsilon}(t)$. Observemos que

$$
\begin{aligned}
& \xi_{\varepsilon}(t)-e^{-\bar{A}_{\varepsilon}^{-}\left(t-t_{0}\right)} \xi_{\varepsilon}\left(t_{0}\right)=z_{\varepsilon}(t)-S_{\varepsilon}^{*}\left(w_{\varepsilon}(t)\right)-e^{-\bar{A}_{\varepsilon}^{-}\left(t-t_{0}\right)}\left[z_{\varepsilon}\left(t_{0}\right)-S_{\varepsilon}^{*}\left(w_{\varepsilon}\left(t_{0}\right)\right)\right] \\
& =\int_{t_{0}}^{t} e^{-\bar{A}_{\bar{\varepsilon}}^{-}(t-s)} G_{\varepsilon}\left(w_{\varepsilon}(s), z_{\varepsilon}(s)\right) d s-S_{\varepsilon}^{*}\left(w_{\varepsilon}(t)\right)+e^{-\bar{A}_{\varepsilon}^{-}\left(t-t_{0}\right)} S_{\varepsilon}^{*}\left(w_{\varepsilon}\left(t_{0}\right)\right) \\
& =\int_{t_{0}}^{t} e^{-\bar{A}_{\varepsilon}^{-}(t-s)} G_{\varepsilon}\left(w_{\varepsilon}(s), z_{\varepsilon}(s)\right) d s-\int_{-\infty}^{t} e^{-\bar{A}_{\bar{\varepsilon}}^{-}(t-s)} G_{\varepsilon}\left(y_{\varepsilon}(s, t), S_{\varepsilon}^{*}\left(y_{\varepsilon}(s, t)\right)\right) d s \\
& +e^{-\bar{A}_{\bar{\varepsilon}}^{-}\left(t-t_{0}\right)} \int_{-\infty}^{t_{0}} e^{-\bar{A}_{\bar{\varepsilon}}^{-}\left(t_{0}-s\right)} G_{\mathcal{\varepsilon}}\left(y_{\mathcal{\varepsilon}}\left(s, t_{0}\right), S_{\mathcal{\varepsilon}}^{*}\left(y_{\mathcal{\varepsilon}}\left(s, t_{0}\right)\right)\right) d s \\
& =\int_{t_{0}}^{t} e^{-\bar{A}_{\varepsilon}^{-}(t-s)}\left[G_{\varepsilon}\left(v_{\varepsilon}(s), z_{\varepsilon}(s)\right)-G_{\varepsilon}\left(y_{\varepsilon}(s, t), S_{\varepsilon}^{*}\left(y_{\varepsilon}(s, t)\right)\right)\right] d s \\
& -\int_{-\infty}^{t_{0}} e^{-\bar{A}_{\varepsilon}^{-}(t-s)}\left[G_{\varepsilon}\left(y_{\varepsilon}(s, t), S_{\varepsilon}^{*}\left(y_{\varepsilon}(s, t)\right)\right)-G_{\varepsilon}\left(y_{\varepsilon}\left(s, t_{0}\right), S_{\varepsilon}^{*}\left(y_{\varepsilon}\left(s, t_{0}\right)\right)\right)\right] d s
\end{aligned}
$$


Logo por (2.35) e (2.36),

$$
\begin{aligned}
\| \xi_{\varepsilon}(t)-e^{-\bar{A}_{\varepsilon}^{-}\left(t-t_{0}\right)} \xi_{\varepsilon}\left(t_{0}\right) & \|_{X_{\varepsilon}^{\frac{1}{2}}} \\
\leqslant & \rho M \int_{t_{0}}^{t} e^{-\beta(t-s)}\left[\left\|w_{\varepsilon}(s)-y_{\varepsilon}(s, t)\right\|_{X_{\varepsilon}^{\frac{1}{2}}}+\left\|z_{\varepsilon}(s)-S_{\varepsilon}^{*}\left(y_{\varepsilon}(s, t)\right)\right\|_{X_{\varepsilon}^{\frac{1}{2}}}\right] d s \\
& +\rho M(1+\Delta) \int_{-\infty}^{t_{0}} e^{-\beta(t-s)}\left\|y_{\varepsilon}(s, t)-y_{\varepsilon}\left(s, t_{0}\right)\right\|_{X_{\varepsilon}^{\frac{1}{2}}} d s \\
& \leqslant \rho M \int_{t_{0}}^{t} e^{-\beta(t-s)}\left\|\xi_{\varepsilon}(s)\right\|_{X_{\varepsilon}^{\frac{1}{2}}} d s+\rho M(1+\Delta) \int_{t_{0}}^{t} e^{-\beta(t-s)}\left\|w_{\varepsilon}(s)-y_{\varepsilon}(s, t)\right\|_{X_{\varepsilon}^{\frac{1}{2}}} d s \\
& +\rho M(1+\Delta) \int_{-\infty}^{t_{0}} e^{-\beta(t-s)}\left\|y_{\varepsilon}(s, t)-y_{\varepsilon}\left(s, t_{0}\right)\right\|_{X_{\varepsilon}^{\frac{1}{2}}} d s \\
& \leqslant \rho M \int_{t_{0}}^{t} e^{-\beta(t-s)}\left\|\xi_{\varepsilon}(s)\right\|_{X_{\varepsilon}^{\frac{1}{2}}} d s \\
& +\rho^{2} M^{2}(1+\Delta) \int_{t_{0}}^{t} e^{-\beta(t-s)} \int_{s}^{t} e^{-(\beta-\rho M(1+\Delta))(\theta-s)}\left\|\xi_{\varepsilon}(\theta)\right\|_{X_{\varepsilon}^{\frac{1}{2}}} d \theta d s \\
& +\rho^{2} M^{3}(1+\Delta) \int_{-\infty}^{t_{0}} e^{-\beta(t-s)} \int_{t_{0}}^{t} e^{-(\beta-\rho M(1+\Delta))(\theta-s)}\left\|\xi^{\varepsilon}(\theta)\right\|_{X_{\varepsilon}^{\frac{1}{2}}} d \theta d s \\
& \leqslant \rho M \int_{t_{0}}^{t} e^{-\beta(t-s)}\left\|\xi_{\varepsilon}(s)\right\|_{X_{\varepsilon}^{\frac{1}{2}}} d s \\
& +\rho^{2} M^{2}(1+\Delta) e^{-\beta t} \int_{t_{0}}^{t} e^{-(\beta-\rho M(1+\Delta)) \theta}\left\|\xi_{\varepsilon}(\theta)\right\|_{X_{\varepsilon}^{\frac{1}{2}}} \int_{t_{0}}^{\theta} e^{(2 \beta-\rho M(1+\Delta)) s} d s d \theta \\
& +\rho^{2} M^{3}(1+\Delta) e^{-\beta t} \int_{t_{0}}^{t} e^{-(\beta-\rho M(1+\Delta)) \theta}\left\|\xi_{\varepsilon}(\theta)\right\|_{X_{\varepsilon}^{\frac{1}{2}}} \int_{-\infty}^{t_{0}} e^{(2 \beta-\rho M(1+\Delta)) s} d s d \theta,
\end{aligned}
$$

de modo que

$$
\begin{aligned}
\| \xi_{\varepsilon}(t) & -e^{-\bar{A}_{\varepsilon}^{-}\left(t-t_{0}\right)} \xi_{\varepsilon}\left(t_{0}\right)\left\|_{X_{\varepsilon}^{\frac{1}{2}}} \leqslant\left[\rho M+\frac{\rho^{2} M^{2}(1+\Delta)}{\beta-\rho M(1+\Delta)}\right] \int_{t_{0}}^{t} e^{-\beta(t-s)}\right\| \xi_{\varepsilon}(s) \|_{X_{\varepsilon}^{\frac{1}{2}}} d s \\
& +\frac{\rho^{2} M^{3}(1+\Delta)}{\beta-\rho M(1+\Delta)} e^{-\beta\left(t-t_{0}\right)} \int_{t_{0}}^{t} e^{-(\beta-\rho M(1+\Delta))\left(\theta-t_{0}\right)}\left\|\xi_{\varepsilon}(\theta)\right\|_{X_{\varepsilon}^{\frac{1}{2}}} d \theta
\end{aligned}
$$

e portanto,

$$
\begin{aligned}
e^{\beta\left(t-t_{0}\right)}\left\|\xi_{\varepsilon}(t)\right\|_{X_{\varepsilon}^{\frac{1}{2}}} & \leqslant M\left\|\xi_{\varepsilon}\left(t_{0}\right)\right\|_{X_{\varepsilon}^{\frac{1}{2}}}+\left[\rho M+\frac{\rho^{2} M^{2}(1+\Delta)}{\beta-\rho M(1+\Delta)}\right] \int_{t_{0}}^{t} e^{\beta\left(s-t_{0}\right)}\left\|\xi_{\varepsilon}(s)\right\|_{X_{\varepsilon}^{\frac{1}{2}}} d s \\
& +\frac{\rho^{2} M^{3}(1+\Delta)}{\beta-\rho M(1+\Delta)} \int_{t_{0}}^{t} e^{-(2 \beta-\rho M(1+\Delta))\left(r-t_{0}\right)} e^{\beta\left(r-t_{0}\right)}\left\|\xi_{\varepsilon}(s)\right\|_{X_{\varepsilon}^{\frac{1}{2}}} d s \\
& \leqslant M\left\|\xi_{\varepsilon}\left(t_{0}\right)\right\|_{X_{\varepsilon}^{\frac{1}{2}}}+\left[\rho M+\frac{\rho^{2} M^{2}(1+\Delta)(1+M)}{\beta-\rho M(1+\Delta)}\right] \int_{t_{0}}^{t} e^{\beta\left(s-t_{0}\right)}\left\|\xi^{\varepsilon}(s)\right\|_{X_{\varepsilon}^{\frac{1}{2}}} d s .
\end{aligned}
$$

Temos ainda pela Desigualdade de Gronwall

$$
\left\|\xi_{\varepsilon}(t)\right\|_{X_{\varepsilon}^{\frac{1}{2}}} \leqslant M\left\|\xi_{\varepsilon}\left(t_{0}\right)\right\|_{X_{\varepsilon}^{\frac{1}{2}}} e^{-\gamma\left(t-t_{0}\right)},
$$

onde

$$
\gamma=\beta-\left[\rho M+\frac{\rho^{2} M^{2}(1+\Delta)(1+M)}{\beta-\rho M(1+\Delta)}\right] .
$$


Logo, $z_{\mathcal{\varepsilon}}(t)=S_{\mathcal{\varepsilon}}^{*}\left(w_{\mathcal{\varepsilon}}(t)\right)$, para todo $t \in \mathbb{R}$, como queríamos.

Finalmente mostremos a continuidade das variedades instáveis dos pontos de equilíbrio. Isto é, se $u_{\mathcal{\varepsilon}}^{*} \in \mathscr{E}_{\mathscr{E}}, \varepsilon \leqslant \bar{\varepsilon}$ é tal que sua variedade instável é dada pelo gráfico de $S_{\mathcal{\varepsilon}}^{*}$, mostremos que

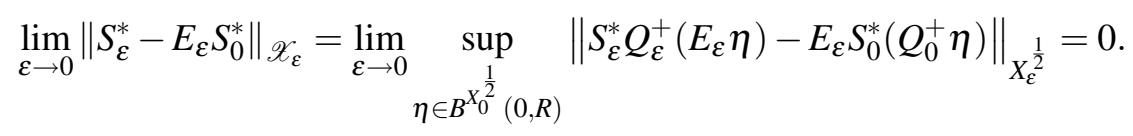

De fato, observemos que

$$
\begin{aligned}
\left\|S_{\varepsilon}^{*} Q_{\varepsilon}^{+}\left(E_{\varepsilon} \eta\right)-E_{\varepsilon} S_{0}^{*}\left(Q_{0}^{+} \eta\right)\right\|_{X_{\varepsilon}^{\frac{1}{2}}} & \leqslant \int_{-\infty}^{\tau}\left\|e^{-\bar{A}_{\varepsilon}^{-}(\tau-s)} G_{\varepsilon}\left(w_{\varepsilon}, S_{\varepsilon}^{*}\left(w_{\varepsilon}\right)\right)-E_{\varepsilon} e^{-\bar{A}_{0}^{-}(\tau-s)} G_{0}\left(w_{0}, S_{0}^{*}\left(w_{0}\right)\right)\right\|_{X_{\varepsilon}^{\frac{1}{2}}} d s \\
& \leqslant \int_{-\infty}^{\tau}\left\|e^{-\bar{A}_{\varepsilon}^{-}(\tau-s)} G_{\varepsilon}\left(w_{\varepsilon}, S_{\varepsilon}^{*}\left(w_{\varepsilon}\right)\right)-e^{-\bar{A}_{\varepsilon}^{-}(\tau-s)} G_{\varepsilon}\left(E_{\varepsilon} w_{0}, E_{\varepsilon} S_{0}^{*}\left(w_{0}\right)\right)\right\|_{X_{\varepsilon}^{\frac{1}{2}}} d s \\
& +\int_{-\infty}^{\tau}\left\|e^{-\bar{A}_{\varepsilon}^{-}(\tau-s)} G_{\varepsilon}\left(E_{\varepsilon} w_{0}, E_{\varepsilon} S_{0}^{*}\left(w_{0}\right)\right)-e^{-\bar{A}_{\varepsilon}^{-}(\tau-s)} E_{\varepsilon} G_{0}\left(w_{0}, S_{0}^{*}\left(w_{0}\right)\right)\right\|_{X_{\varepsilon}^{\frac{1}{2}}} d s \\
& +\int_{-\infty}^{\tau}\left\|e^{-\bar{A}_{\bar{\varepsilon}}^{-}(\tau-s)} E_{\varepsilon} G_{0}\left(w_{0}, S_{0}^{*}\left(w_{0}\right)\right)-E_{\varepsilon} e^{-\bar{A}_{0}^{-}(\tau-s)} G_{0}\left(w_{0}, S_{0}^{*}\left(w_{0}\right)\right)\right\|_{X_{\varepsilon}^{\frac{1}{2}}} d s .
\end{aligned}
$$

Denotando as três últimas integrais por $I_{1}, I_{2}$ e $I_{3}$, respectivamente, temos

$$
\begin{aligned}
I_{1} & \leqslant \int_{-\infty}^{\tau} M e^{-\beta(\tau-s)}(\tau-s)^{-\frac{1}{2}} \rho\left[(1+\Delta)\left\|w_{\varepsilon}(s)-E_{\varepsilon} w_{0}(s)\right\|_{X_{\varepsilon}^{\frac{1}{2}}}+\left\|S_{\varepsilon}^{*}-E_{\varepsilon} S_{0}^{*}\right\|_{\mathscr{X}_{\varepsilon}}\right] d s \\
& \leqslant \rho M \beta^{-\frac{1}{2}} \Gamma\left(\frac{1}{2}\right)\left\|S_{\varepsilon}^{*}-E_{\varepsilon} S_{0}^{*}\right\|_{\mathscr{X}_{\varepsilon}}+\rho M(1+\Delta) \int_{-\infty}^{\tau} e^{-\beta(\tau-s)}(\tau-s)^{-\frac{1}{2}}\left\|w_{\varepsilon}(s)-E_{\varepsilon} w_{0}(s)\right\|_{X_{\varepsilon}^{\frac{1}{2}}} d s .
\end{aligned}
$$

Como a aplicação $Q_{0}^{+} X_{0}^{\frac{1}{2}} \ni w_{0} \mapsto G_{0}\left(w_{0}, S^{*}\left(w_{0}\right)\right)$ é contínua, ela leva subconjuntos limitados de $Q_{0}^{+} X_{0}^{\frac{1}{2}}$ em subconjuntos relativamente compactos de $X_{0}^{\frac{1}{2}}$. Observemos ainda que $G_{\varepsilon} \stackrel{E E}{\longrightarrow} G_{0}$, e portanto, $I_{2} \rightarrow 0$ quando $\varepsilon \rightarrow 0$. Finalmente, como $G_{0}\left(w_{0}, S_{0}^{*}\left(w_{0}\right)\right)$ está em um conjunto compacto de $X_{0}^{\frac{1}{2}}$, garantimos, como no Teorema 2.14 , que $I_{3} \rightarrow 0$ quando $\varepsilon \rightarrow 0$. Logo,

$$
\begin{aligned}
\left\|S_{\varepsilon}^{*} Q_{\varepsilon}^{+}\left(E_{\varepsilon} \eta\right)-E_{\varepsilon} S_{0}^{*}\left(Q_{0}^{+} \eta\right)\right\|_{X_{\varepsilon}^{\frac{1}{2}} \leqslant} & o(1)+\rho M \beta^{-\frac{1}{2}} \Gamma\left(\frac{1}{2}\right)\left\|S_{\varepsilon}^{*}-S_{0}^{*}\right\|_{\mathscr{X}_{\varepsilon}} \\
& +\rho M(1+\Delta) \int_{-\infty}^{\tau} e^{-\beta(\tau-s)}(\tau-s)^{-\frac{1}{2}}\left\|w_{\varepsilon}(s)-E_{\varepsilon} w_{0}(s)\right\|_{X_{\varepsilon}^{\frac{1}{2}}} d s .
\end{aligned}
$$

Portanto é suficiente estimarmos $\left\|w_{\varepsilon}(t)-E_{\varepsilon} w_{0}(t)\right\|_{X_{\varepsilon}^{\frac{1}{2}}}$. Como $\eta \in B_{X_{0}^{\frac{1}{2}}}(0, R)$, de maneira aná- 
loga, obtemos

$$
\begin{aligned}
\left\|w_{\varepsilon}(t)-E_{\varepsilon} w_{0}(t)\right\|_{X_{\varepsilon}^{\frac{1}{2}} \leqslant} \leqslant & \left\|e^{-\bar{A}_{\varepsilon}^{+}(t-\tau)} E_{\varepsilon} \eta-E_{\varepsilon} e^{-\bar{A}_{0}^{+}(t-\tau)} \eta\right\|_{X_{\varepsilon}^{\frac{1}{2}}} \\
& +\int_{t}^{\tau}\left\|e^{-\bar{A}_{\varepsilon}^{+}(t-s)} H_{\varepsilon}\left(w_{\varepsilon}, S_{\varepsilon}^{*}\left(w_{\varepsilon}\right)\right)-e^{-\bar{A}_{\varepsilon}^{+}(t-s)} H_{\varepsilon}\left(E_{\varepsilon} w_{0}, E_{\varepsilon} S_{0}^{*}\left(w_{0}\right)\right)\right\|_{X_{\varepsilon}^{\frac{1}{2}}} d s \\
& +\int_{t}^{\tau}\left\|e^{-\bar{A}_{\varepsilon}^{+}(t-s)} H_{\varepsilon}\left(E_{\varepsilon} w_{0}, E_{\varepsilon} S_{0}^{*}\left(w_{0}\right)\right)-e^{-\bar{A}_{\varepsilon}^{+}(t-s)} E_{\varepsilon} H_{0}\left(w_{0}, S_{0}^{*}\left(w_{0}\right)\right)\right\|_{X_{\varepsilon}^{\frac{1}{2}}} d s \\
& +\int_{t}^{\tau}\left\|e^{-\bar{A}_{\varepsilon}^{+}(t-s)} E_{\varepsilon} H_{0}\left(w_{0}, S_{0}^{*}\left(w_{0}\right)\right)-E_{\varepsilon} e^{-\bar{A}_{0}^{+}(t-s)} H_{0}\left(w_{0}, S_{0}^{*}\left(w_{0}\right)\right)\right\|_{X_{\varepsilon}^{\frac{1}{2}}} d s \\
\leqslant & o(1)+\rho M \int_{t}^{\tau} e^{\beta(t-s)} d s\left\|S_{\varepsilon}^{*}-E_{\varepsilon} S_{0}^{*}\right\|_{\mathscr{X}_{\varepsilon}} \\
& +\rho M(1+\Delta) \int_{t}^{\tau} e^{\beta(t-s)}\left\|w_{\varepsilon}(s)-E_{\varepsilon} w_{0}(s)\right\|_{X_{\varepsilon}^{\frac{1}{2}}} d s .
\end{aligned}
$$

Seja $\phi(t)=e^{\beta(\tau-t)}\left\|w_{\varepsilon}(t)-E_{\varepsilon} w_{0}(t)\right\|_{X_{\varepsilon}^{\frac{1}{2}}}$. Então

$$
\phi(t) \leqslant o(1)+\rho M \int_{t}^{\tau} e^{\beta(\tau-s)} d s\left\|S_{\mathcal{\varepsilon}}^{*}-E_{\varepsilon} S_{0}^{*}\right\|_{\mathscr{X}_{\varepsilon}}+\rho M(1+\Delta) \int_{t}^{\tau} \phi(s) d s
$$

e obtemos

$$
\left\|w_{\varepsilon}(t)-E_{\varepsilon} w_{0}(t)\right\|_{X_{\varepsilon}^{\frac{1}{2}}} \leqslant \rho M\left[o(1)+\beta^{-1}\left\|S_{\varepsilon}^{*}-E_{\varepsilon} S_{0}^{*}\right\|_{\mathscr{X}_{\varepsilon}}\right] e^{[\rho M(1+\Delta)-\beta](\tau-t)} .
$$

Portanto

$$
\begin{aligned}
&\left\|S_{\varepsilon}^{*}\left(E_{\varepsilon} \eta\right)-E_{\varepsilon} S_{0}^{*}(\eta)\right\|_{X_{\varepsilon}^{\frac{1}{2}}} \leqslant o(1)+\rho M \beta^{-\frac{1}{2}} \Gamma\left(\frac{1}{2}\right)\left\|S_{\varepsilon}^{*}-E_{\varepsilon} S_{0}^{*}\right\|_{\mathscr{X}_{\varepsilon}} \\
&+ \rho M(1+\Delta) \int_{-\infty}^{\tau} e^{[-2 \beta+\rho M(1+\Delta)](\tau-s)}(\tau-s)^{-\frac{1}{2}} d s \\
& {\left[o(1)+\rho M \beta^{-1}\left\|S_{\varepsilon}^{*}-E_{\varepsilon} S_{0}^{*}\right\|_{\mathscr{X}_{\varepsilon}}\right] } \\
& \quad \leqslant o(1)+\rho M \beta^{-\frac{1}{2}} \Gamma\left(\frac{1}{2}\right)\left\|S_{\varepsilon}^{*}-E_{\varepsilon} S_{0}^{*}\right\|_{\mathscr{X}_{\varepsilon}} \\
&+\left[\rho M(1+\Delta)(2 \beta-\rho M(1+\Delta))^{-\frac{1}{2}} \Gamma\left(\frac{1}{2}\right) \rho M \beta^{-1}\right]\left\|S_{\varepsilon}^{*}-E_{\varepsilon} S_{0}^{*}\right\|_{\mathscr{X}_{\varepsilon}}
\end{aligned}
$$

Assim,

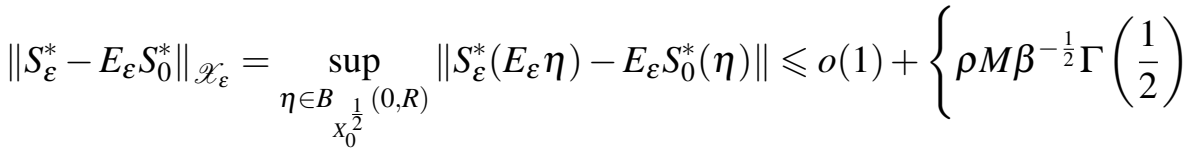

$$
\begin{aligned}
& \left.+\left[\rho^{2} M^{2} \beta^{-1}(1+\Delta)(2 \beta-\rho M(1+\Delta))^{-\frac{1}{2}} \Gamma\left(\frac{1}{2}\right)\right]\right\}\left\|S_{\varepsilon}^{*}-E_{\varepsilon} S_{0}^{*}\right\|_{\mathscr{X}_{\varepsilon}},
\end{aligned}
$$

e portanto $\left\|S_{\varepsilon}^{*}-E_{\varepsilon} S_{0}^{*}\right\|_{\mathscr{X}_{\varepsilon}} \rightarrow 0$ quando $\varepsilon \rightarrow 0$, como queríamos. 
Corolário 2.37. Nas hipóteses do Teorema anterior existe $\delta>0$ tal que

$$
\mathrm{W}_{\varepsilon, \text { loc }}^{u}=\left\{\left(w_{\varepsilon}, z_{\varepsilon}\right) \in X_{\varepsilon}^{\frac{1}{2}} \oplus\left(I-Q_{\varepsilon}^{+}\right) X_{\varepsilon}^{\frac{1}{2}}:\left(w_{\varepsilon}, z_{\varepsilon}\right) \in W_{\varepsilon}^{u},\left\|w_{\varepsilon}\right\|_{X_{\varepsilon}^{\frac{1}{2}}}+\left\|z_{\varepsilon}\right\|_{X_{\varepsilon}^{\frac{1}{2}}}<\delta\right\}
$$

é dada por

$$
\mathrm{W}_{\varepsilon, \mathrm{loc}}^{u}=\left\{\left(w_{\varepsilon}, S_{\varepsilon}^{*}\left(w_{\varepsilon}\right)\right): w_{\varepsilon} \in X_{\varepsilon}^{\frac{1}{2}}\right\} \cap\left\{\left(w_{\varepsilon}, z_{\varepsilon}\right) \in X_{\varepsilon}^{\frac{1}{2}} \oplus\left(I-Q_{\varepsilon}^{+}\right) X_{\varepsilon}^{\frac{1}{2}}:\left\|w_{\varepsilon}\right\|_{X_{\varepsilon}^{\frac{1}{2}}}+\left\|z_{\varepsilon}\right\|_{X_{\varepsilon}^{\frac{1}{2}}}<\delta\right\} .
$$

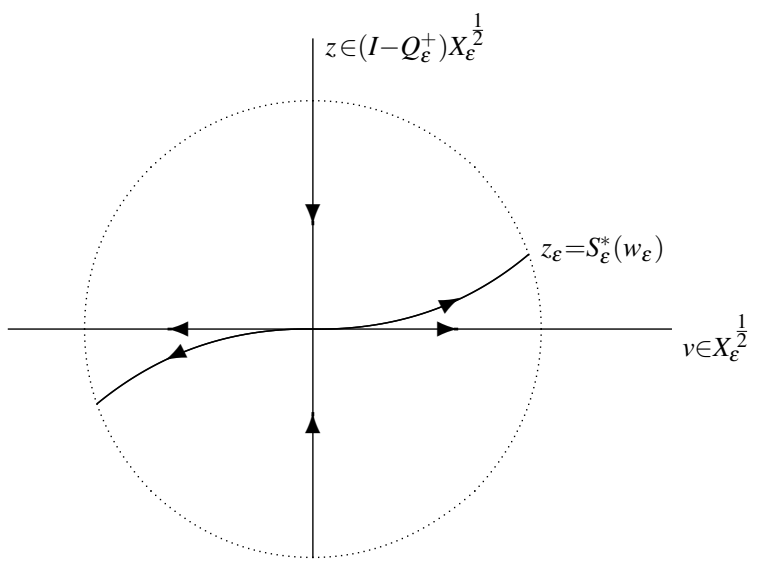

Figura 2: $W_{\varepsilon, \text { loc }}^{u}\left(u_{*}^{\varepsilon}\right)$

Como conseqüência imediata da Proposição 2.36, temos

Corolário 2.38. Assumamos que as condições da Proposição 2.36 estejam satisfeitas, $\mathscr{E} 0=$ $\left\{u_{0,1}^{*}, \cdots, u_{0, n_{0}}^{*}\right\}$ onde cada $u_{0, i}^{*}, i=1, \cdots, n_{0}$ é hiperbólico. Então existem $\boldsymbol{\delta}>0, \varepsilon_{0}>0$ tais que $\mathscr{E}_{\varepsilon}=$ $\left\{u_{\varepsilon, 1}^{*}, \cdots, u_{\varepsilon, n_{0}}^{*}\right\}, \varepsilon \in\left[0, \varepsilon_{0}\right]$, e suas variedades instáveis locais $\mathrm{W}_{\varepsilon}^{u}\left(u_{\varepsilon, j}^{*}\right), j=1, \ldots, n_{0}$, satisfazem

$$
\mathrm{W}_{\varepsilon}^{u}\left(u_{\varepsilon, j}^{*}\right) \stackrel{E}{\longrightarrow} \mathrm{W}_{0}^{u}\left(u_{0, j}^{*}\right)
$$

2.2.4 Semicontinuidade inferior dos atratores

Nas hipóteses das seções anteriores e supondo adicionalmente que os semigrupos $\left\{T_{\varepsilon}(t) ; t \geqslant 0\right\}$ possuem estrutura gradiente,

Teorema 2.39. A família de atratores $\left\{\mathscr{A}_{\varepsilon}: \varepsilon \in\left(0, \varepsilon_{0}\right]\right\}$ é semicontínua inferiormente em $\varepsilon=0$. 
Demonstração: Seja $u \in \mathscr{A}_{0}$. Como $T_{0}(t)$ é gradiente, temos $\mathscr{A}_{0}=\bigcup_{u_{0}^{*} \in \mathscr{E}_{0}} \mathrm{~W}^{u}\left(u_{0}^{*}\right)$, de modo que $u \in \mathrm{W}^{u}\left(u_{0}^{*}\right)$, para algum $u_{0}^{*} \in \mathscr{E}_{0}$. Sejam $\tau \in \mathbb{R}$ e $v \in \mathrm{W}^{u}\left(u_{0}^{*}\right)$ tais que $T_{0}(\tau) v=u$. Seja ainda $u_{\varepsilon}^{*}$ com $u_{\varepsilon}^{*} \stackrel{E}{\longrightarrow} u_{0}^{*}$. Da convergência das variedades invariantes segue a existência de uma seqüência $v_{\varepsilon} \in \mathrm{W}_{\varepsilon}^{u}\left(u_{\varepsilon}^{*}\right)$ tal que $v_{\varepsilon} \stackrel{E}{\longrightarrow} v$. Finalmente, da continuidade dos semigrupos não-lineares, temos que $T_{\varepsilon}(\tau) v_{\varepsilon} \stackrel{E}{\longrightarrow} T_{0}(\tau) v=u$. Para concluir a demonstração, resta-nos apenas observar que se $u_{\varepsilon}=T_{\varepsilon}(\tau) v_{\varepsilon}$, então $u_{\varepsilon} \in \mathscr{A}_{\varepsilon}$, pois $v_{\varepsilon} \in \bigcup_{u_{\varepsilon}^{*} \in \mathscr{E}_{\varepsilon}} \mathrm{W}^{u}\left(u_{\varepsilon}^{*}\right)=\mathscr{A}_{\varepsilon}$ e $\mathscr{A}_{\varepsilon}$ é invariante.

$u$

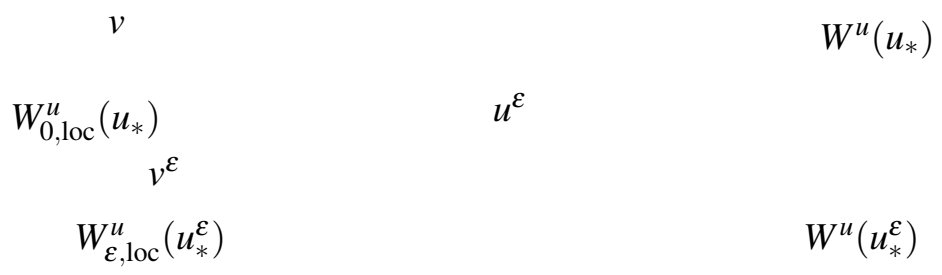

Figura 3: Semicontinuidade inferior dos atratores

Corolário 2.40. A família de atratores $\left\{\mathscr{A}_{\varepsilon}: \varepsilon \in\left(0, \varepsilon_{0}\right]\right\}$ é contínua em $\varepsilon=0$. 


\title{
Segunda Parte - Aplicações
}

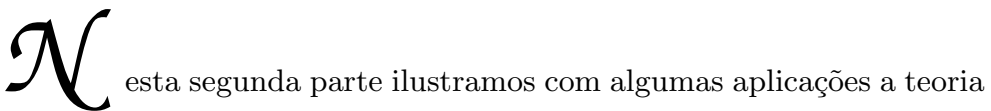 \\ abstrata desenvolvida na primeira parte. Analisamos alguns exemplos \\ dos chamados domínios finos e mostramos como obter a continuidade \\ de sua dinâmica assintótica. Na literatura existe um tratamento sepa- \\ rado para cada caso e aqui colocamos alguns exemplos representativos \\ dessa classe sob a perspectiva apresentada anteriormente.
}





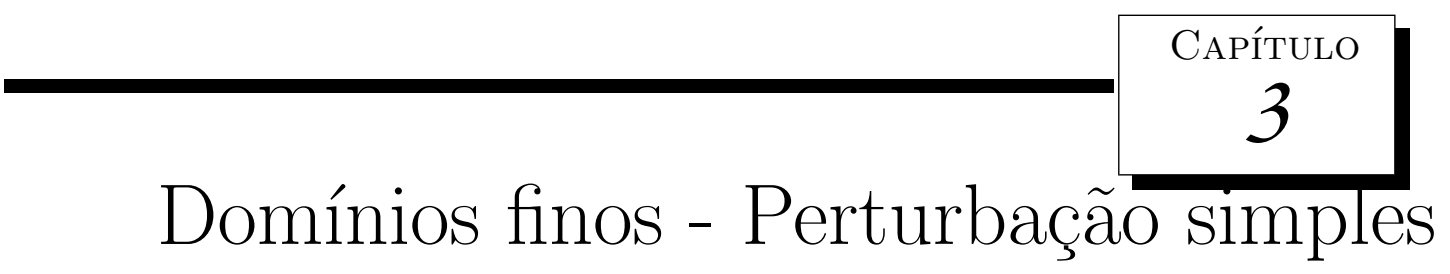

Seja $\Omega \subset \mathbb{R}^{m+n}$ um domínio limitado e regular (fronteira pelo menos de classe $C^{2}$ ). Escreveremos os pontos de $\mathbb{R}^{m+n}$ como $(x, y)$, onde $x \in \mathbb{R}^{m}$ e $y \in \mathbb{R}^{n}$. Denotaremos por $\nabla_{x}$ (resp. $\nabla_{y}$ ) os operadores gradientes atuando em $\mathbb{R}^{m} \subset \mathbb{R}^{m+n}$ (resp. $\mathbb{R}^{n} \subset \mathbb{R}^{m+n}$ ). Analogamente, $\Delta_{x}$ (resp. $\Delta_{y}$ ) denota o operador de Laplace atuando em $\mathbb{R}^{m}$ (resp. $\mathbb{R}^{n}$ ).

Começaremos estudando o comportamento dinâmico das soluções do problema de reaçãodifusão

$$
\begin{array}{ll}
u_{t}=\Delta u-u+f(u), & (0, \infty) \times \Omega \\
\frac{\partial u}{\partial \eta}=0, & (0, \infty) \times \partial \Omega
\end{array}
$$

com respeito a perturbações singulares do domínio $\Omega$, assumindo que a não-linearidade $f$ seja espacialmente homogênea e satisfaça certas condições de crescimento e coercividade.

Comprimindo-se o domínio $\Omega$ por um fator $0<\varepsilon<1$ na direção $y$, obtemos o domínio comprimido $\Omega_{\varepsilon}$. Mais precisamente, colocando $\Omega_{\varepsilon}:=T_{\varepsilon}(\Omega)$, onde $T_{\varepsilon}: \mathbb{R}^{m+n} \rightarrow \mathbb{R}^{m+n}$ é a transformação $T_{\varepsilon}(x, y)=(x, \varepsilon y)$, nós consideramos neste domínio comprimido a família de problemas de reaçãodifusão

$$
\begin{array}{ll}
u_{t}=\Delta u-u+f(u), & (0, \infty) \times \Omega_{\varepsilon} \\
\frac{\partial u}{\partial \eta_{\varepsilon}}=0, & (0, \infty) \times \partial \Omega_{\varepsilon}
\end{array}
$$

onde $\eta_{\varepsilon}$ é o vetor normal unitário exterior a $\partial \Omega_{\varepsilon}$, e $f: \mathbb{R} \rightarrow \mathbb{R}$ é uma não linearidade satisfazendo certas condições de crescimento que assegurem que a equação $\left(\tilde{P}_{\varepsilon}\right)$ gere um semigrupo $\left\{\tilde{T}_{\varepsilon}(t) ; t \geqslant 0\right\}$ de operadores (não lineares) em $H^{1}\left(\Omega_{\varepsilon}\right)$. Aqui o domínio $\Omega_{\varepsilon}$ varia de acordo com o parâmetro $\varepsilon$. Nosso interesse é analisar o que ocorre com a dinâmica de $\left(\tilde{P}_{\varepsilon}\right)$ quando $\varepsilon \rightarrow 0$.

Neste caso podemos reescalar o domínio $\Omega_{\varepsilon}$ obtendo o domínio fixado inicialmente $\Omega$. A equação $\left(\tilde{P}_{\varepsilon}\right)$ pode ser escrita de maneira equivalente, onde o parâmetro $\varepsilon$ é incorporado à equação

$$
\begin{array}{ll}
u_{t}=\Delta_{x} u+\frac{1}{\varepsilon^{2}} \Delta_{y} u-u+f(u), & (0, \infty) \times \Omega \\
\frac{\partial u}{\partial \eta_{x}}+\frac{1}{\varepsilon^{2}} \frac{\partial u}{\partial \eta_{y}}=0, & (0, \infty) \times \partial \Omega
\end{array}
$$


onde $\eta=\left(\frac{\partial u}{\partial \eta_{x}}, \frac{\partial u}{\partial \eta_{y}}\right)$ é o vetor normal unitário exterior a $\partial \Omega$.

Apresentaremos a relação entre os problemas $\left(\tilde{P}_{\varepsilon}\right)$ e $\left(P_{\mathcal{E}}\right)$. Estabelecida uma equivalência entre esses problemas podemos estudar a equação de evolução abstrata associada a $\left(P_{\varepsilon}\right)$.

Fixado $\varepsilon>0$, definimos a aplicação $\Phi_{\varepsilon}: L^{2}\left(\Omega_{\varepsilon}\right) \rightarrow L^{2}(\Omega)$ dada por $u \mapsto u \circ T_{\varepsilon}$. Observemos que este isomorfismo se restringe a um isomorfismo de $H^{1}\left(\Omega_{\varepsilon}\right)$ sobre $H^{1}(\Omega)$. Este isomorfismo nos fornece uma conjugação entre os problemas $\left(\tilde{P}_{\varepsilon}\right)$ e $\left(P_{\varepsilon}\right)$ da seguinte forma:

Se $\left\{\tilde{T}_{\mathcal{E}}(t) ; t \geqslant 0\right\}$ é o semigrupo de soluções associado ao problema $\left(\tilde{P}_{\mathcal{E}}\right)$, então

$$
T_{\varepsilon}(t)(\cdot):=\Phi_{\varepsilon} \tilde{T}_{\varepsilon}(t) \Phi_{\varepsilon}^{-1}(\cdot),
$$

define um semigrupo de soluções em um subespaço vetorial fechado de $H^{1}(\Omega)$. Não é difícil verificar que $\left\{T_{\mathcal{\varepsilon}}(t) ; t \geqslant 0\right\}$ é o semigrupo de soluções associado ao problema $\left(P_{\varepsilon}\right)$.

No sentido de capturarmos o comportamento limite do problema $\left(\tilde{P}_{\varepsilon}\right)$, faz-se necessário considerarmos espaços nos quais as funções $h$ definidas em $\Omega_{\varepsilon}$, e que são independentes da variável $y$, possuam norma que não tendam a zero $\operatorname{com} \varepsilon$. Assim, para compararmos os semigrupos $\left\{\tilde{T}_{\mathcal{E}}(t) ; t \geqslant 0\right\}$ e $\left\{T_{\mathcal{E}}(t) ; t \geqslant 0\right\}$ introduzimos o seguinte marco funcional.

Dado $\varepsilon \in(0,1)$, consideremos os espaços, $U_{\varepsilon}:=L^{2}\left(\Omega_{\varepsilon}\right)$ munido da norma $\|u\|_{U_{\varepsilon}}=\varepsilon^{\frac{-n}{2}}\|u\|_{L^{2}\left(\Omega_{\varepsilon}\right)}$ e $U_{\varepsilon}^{1}:=H^{1}\left(\Omega_{\varepsilon}\right)$ munido da norma $\|u\|_{U_{\varepsilon}^{1}}=\varepsilon^{\frac{-n}{2}}\|u\|_{H^{1}\left(\Omega_{\varepsilon}\right)}$. Introduzindo em $H^{1}(\Omega)$ a norma equivalente

$$
\|u\|_{\varepsilon}:=\left(\int_{\Omega}\left(\left|\nabla_{x} u\right|^{2} \frac{1}{\varepsilon^{2}}\left|\nabla_{y} u\right|^{2}+|u|^{2}\right) d x d y\right)^{\frac{1}{2}}
$$

vê-se que $\Phi_{\varepsilon}: U_{\varepsilon}^{1} \rightarrow H_{\varepsilon}^{1}(\Omega)$ é uma isometria, onde $H_{\varepsilon}^{1}(\Omega):=\left(H^{1}(\Omega),\|\cdot\|_{\varepsilon}\right) . \operatorname{Em} X=: L^{2}(\Omega)$ usaremos o produto interno usual $\langle u, v\rangle=\int_{\Omega} u v d x d y$.

Observemos que $H_{\varepsilon}^{1}(\Omega)$ é um espaço de Hilbert com produto interno

$$
\begin{gathered}
a_{\varepsilon}: H_{\varepsilon}^{1}(\Omega) \times H_{\varepsilon}^{1}(\Omega) \rightarrow \mathbb{R} \\
a_{\varepsilon}(u, v):=\int_{\Omega}\left(\nabla_{x} u \cdot \nabla_{x} v+\frac{1}{\varepsilon^{2}} \nabla_{y} u \cdot \nabla_{y} v+u \cdot v\right) d x d y
\end{gathered}
$$

que induz um operador linear $A_{\varepsilon}: D\left(A_{\varepsilon}\right) \subset X \rightarrow X$ auto-adjunto, positivo, com resolvente compacto, definido por $a_{\varepsilon}(u, v)=\left\langle A_{\varepsilon} u, v\right\rangle, \forall v \in H_{\varepsilon}^{1}(\Omega)$, segundo a proposição

Proposição 3.1 (Lax-Milgran). Sejam $V, H$ espaços de Hilbert com $V \hookrightarrow H$. Assuma que a : $V \times V \rightarrow \mathbb{R}$ é uma forma bilinear contínua e coerciva. Então existe um único operador linear limitado $B \in \mathscr{L}\left(V, V^{*}\right)$ tal que

$$
a(u, v)=\langle B u, v\rangle, \quad u, v \in V
$$

e as seguintes propriedades são satisfeitas: 
i. B é um isomorfismo de $V$ sobre $V^{*}$.

ii. A restrição $B_{H}$ do operador $B$ a $D\left(B_{H}\right)=\{v \in V: B v \in H\}$ é um operador linear fechado em $H$ (chamado de $H$-realização).

iii. Se a forma bilinear a é simétrica então $B_{H}$ é auto-adjunto.

iv. Se, além disso, a imersão $V \hookrightarrow H$ é compacta, então o operador $B_{H}$ possui resolvente compacto.

Agora observemos que ao fazer $\varepsilon \rightarrow 0$ na expressão de $a_{\varepsilon}$, se $u \in H^{1}(\Omega)$, então

$$
\lim _{\varepsilon \rightarrow 0} a_{\mathcal{\varepsilon}}(u, u)=\left\{\begin{array}{cl}
\int_{\Omega}\left(\left|\nabla_{x} u\right|^{2}+|u|^{2}\right) d x d y & \text { se } \nabla_{y} u=0 \\
\infty & \text { se } \nabla_{y} u \neq 0 .
\end{array}\right.
$$

Logo na tentativa de estabelecermos um limite formal para a equação $\left(P_{\varepsilon}\right)$ somos levados a considerar a "forma bilinear limite", $a_{0}: H_{s}^{1}(\Omega) \times H_{s}^{1}(\Omega) \rightarrow \mathbb{R}$, definida em um subespaço vetorial de $H^{1}(\Omega) \times H^{1}(\Omega)$, onde $H_{s}^{1}(\Omega):=\left\{u \in H^{1}(\Omega): \nabla_{y} u=0\right\}$. Definimos $a_{0}$ por

$$
a_{0}(u, v):=\int_{\Omega}(\nabla u \cdot \nabla v+u v) d x d y .
$$

Teorema 3.2. O conjunto $H_{s}^{1}(\Omega)$ é um subespaço vetorial fechado de $H^{1}(\Omega)$. Além disso, $H_{s}^{1}(\Omega)$ possui dimensão infinita.

Demonstração: Ver [39].

Se denotarmos por $L_{s}^{2}:=$ fecho de $H_{s}^{1}(\Omega)$ em $L^{2}(\Omega)$, a Proposição 3.1 garante também que $a_{0}$ define um operador linear $A_{0}: D\left(A_{0}\right) \subset L_{s}^{2} \rightarrow L_{s}^{2}$ auto-adjunto, positivo, com resolvente compacto, definido por

$$
a_{0}(u, v)=\left\langle A_{0} u, v\right\rangle_{L_{s}^{2}, L_{s}^{2}}=\left\langle A_{0} u, v\right\rangle, \forall v \in H_{s}^{1} .
$$

Proposição 3.3. Dado $\varepsilon \in[0,1]$ o operador $A_{\varepsilon}: D\left(A_{\varepsilon}\right) \subset L^{2}(\Omega) \rightarrow L^{2}(\Omega)$, (substituindo $L^{2}(\Omega)$ por $L_{s}^{2}$, quando $\varepsilon=0$ ) é setorial valendo a estimativa

$$
\left\|\left(A_{\varepsilon}+\mu\right)^{-1}\right\|_{\mathscr{L}\left(L^{2}(\Omega)\right)} \leqslant \frac{\mathrm{M}}{|\mu+1|}, \quad \text { para todo } \mu \in \Sigma_{-1, \phi},
$$

onde $\mathrm{M}$ é uma constante independente de $\varepsilon$ e $0<\phi<\frac{\pi}{2}$.

Demonstração: Como $\left\langle-A_{\varepsilon} u, u\right\rangle \leqslant-\langle u, u\rangle$, para todo $u \in H_{\varepsilon}^{1}(\Omega)$, temos que $W\left(-A_{\varepsilon}\right) \subset(-\infty,-1]$. Já que $\Sigma:=\mathbb{C} \backslash(-\infty,-1] \subset \mathbb{C} \backslash W\left(-A_{\varepsilon}\right)$ é aberto e conexo, e ainda $0 \in \Sigma \cap \rho\left(-A_{\varepsilon}\right)$, o Teorema 1.10 garante que se $\mu \in \Sigma$, temos

$$
\left\|\left(\mu+A_{\varepsilon}\right)^{-1}\right\|_{\mathscr{L}\left(L^{2}(\Omega)\right)} \leqslant \frac{1}{\operatorname{dist}\left(\mu, W\left(-A_{\varepsilon}\right)\right)} .
$$


Logo, se $0<\phi<\frac{\pi}{2}$ e $\mu \in \Sigma_{-1, \phi}=\{w \in \mathbb{C}:|\arg (\mathrm{w}+1)|<\pi-\phi\}$, temos

$$
\operatorname{dist}\left(\mu, W\left(-A_{\varepsilon}\right)\right) \geqslant \operatorname{dist}(\mu,(-\infty,-1])=|\mu+1| \sin (\pi-\arg (\mu+1)) \geqslant|\mu+1| \sin \phi,
$$

obtendo assim

$$
\left\|\left(\mu+A_{\varepsilon}\right)^{-1}\right\|_{\mathscr{L}\left(L^{2}(\Omega)\right)} \leqslant \frac{M}{|\mu+1|}, \quad \text { para todo } \mu \in \Sigma_{-1, \phi}
$$

onde $M=\frac{1}{\sin \phi}$ independe de $\varepsilon$.

Nas condições acima, para cada $0 \leqslant \varepsilon<1$ os espaços de potência fracionária $X_{\varepsilon}^{\alpha}, \alpha \in \mathbb{R}$, associados ao operador $A_{\varepsilon}$, (ver Henry [34]), estão bem definidos e verificam as seguintes propriedades de imersão

Proposição 3.4. Para cada $0 \leqslant \varepsilon<1$ sejam $X_{\varepsilon}^{\alpha}$ os espaços de potência fracionária associados ao operador $A_{\varepsilon}$. Então se $0<\varepsilon<1$, para cada $0 \leqslant \alpha<1$, temos que $X_{\varepsilon}^{\alpha} \hookrightarrow H^{2 \alpha}(\Omega)$, e em particular

$$
X_{\varepsilon}^{1}=D\left(A_{\varepsilon}\right), \quad X_{\varepsilon}^{\frac{1}{2}}=H_{\varepsilon}^{1}(\Omega), \quad X_{\varepsilon}^{0}=L^{2}(\Omega) .
$$

Adicionalmente

$$
X_{0}^{1}=D\left(A_{0}\right), \quad X_{0}^{\frac{1}{2}}=H_{s}^{1}(\Omega), \quad X_{0}^{0}=L_{s}^{2}(\Omega) .
$$

Demonstração: Ver [8].

Consideremos agora a seguinte hipótese sobre a não-linearidade $f$.

(HC) Se $N=m+n>2$, suponhamos que $f \in C^{2}(\mathbb{R})$ e para algum $C>0,\left|f^{\prime}(s)\right| \leqslant C\left(|s|^{\frac{4}{N-2}}+1\right)$ para todo $s \in \mathbb{R}$. Além disso, supomos também que

$$
\lim \sup _{|s| \rightarrow \infty} \frac{f(s)}{s}<0 .
$$

Em [9] os autores mostram que sob estas condições o problema $\left(\tilde{P}_{\varepsilon}\right)$ é globalmente $(t \geqslant 0)$ bem posto em $H^{1}\left(\Omega_{\varepsilon}\right)$, e mostram ainda a existência de atratores $\tilde{\mathscr{A}}_{\varepsilon} \subset H^{1}\left(\Omega_{\varepsilon}\right)$ (união de órbitas limitadas) uniformemente limitados em $L^{\infty}\left(\Omega_{\varepsilon}\right)$. Mais precisamente

Teorema 3.5. Suponha que $f$ satisfaça as hipóteses acima. Então existe $\varepsilon_{0}>0$ tal que os atratores $\mathscr{A}_{\mathcal{\varepsilon}} \subset X_{\mathcal{\varepsilon}}^{\frac{1}{2}}$ dos semigrupos não-lineares $\left\{T_{\mathcal{\varepsilon}}(t) ; t \geqslant 0\right\}, \varepsilon \in[0, \bar{\varepsilon}]$, associados aos problemas (3.2) e (3.5) satisfazem

$$
\sup _{\varepsilon \in\left[0, \varepsilon_{0}\right]} \sup _{w \in \mathscr{A} \varepsilon}\|w\|_{X_{\varepsilon}^{\frac{1}{2}}}<\infty
$$

$e$

$$
\sup _{\varepsilon \in\left[0, \varepsilon_{0}\right]} \sup _{w \in \mathscr{A}_{\varepsilon}}\|w\|_{L^{\infty}(\Omega)}<\infty
$$


Demonstração: Ver [9]

Isto nos permite "cortar" a não linearidade $f$ fora de um intervalo suficientemente grande, de modo que ela e suas derivadas até segunda ordem permaneçam limitadas, sem com isso, modificar os atratores $\mathscr{A}_{\mathcal{E}}$.

(HC*) Sem perda de generalidade assumimos a partir de agora, que a não linearidade $f$ é uma função Lipschitz contínua com derivadas limitadas até segunda ordem, satisfazendo

$$
\lim \sup _{|s| \rightarrow \infty} \frac{f(s)}{s}<0 .
$$

Lema 3.6. Dado $\varepsilon>0$, consideremos a seguinte aplicação $F_{\varepsilon}: X_{\varepsilon}^{\frac{1}{2}} \rightarrow X_{\varepsilon}^{0}$ dada por $F_{\varepsilon}:=\Phi_{\varepsilon} \circ \tilde{F}_{\varepsilon} \circ$ $\Phi_{\varepsilon}^{-1}$, onde $\tilde{F}_{\varepsilon}: U_{\varepsilon}^{1} \rightarrow X_{\varepsilon}^{0}$ é o operador de Nemitsküi associado a $f$. Então $F_{\varepsilon}$ é Lipschitz contínua.

Demonstração: A aplicação $F_{\varepsilon}$ está bem definida já que

$$
H^{1}(\Omega) \stackrel{\Phi_{\varepsilon}^{-1}}{\longrightarrow} H^{1}\left(\Omega_{\varepsilon}\right) \stackrel{\tilde{F}_{\varepsilon}}{\longrightarrow} L^{2}\left(\Omega_{\varepsilon}\right) \stackrel{\Phi_{\varepsilon}}{\longrightarrow} L^{2}(\Omega) .
$$

Além disso $F_{\varepsilon}$ não depende do parâmetro $\varepsilon$, já que para quaisquer que sejam $u \in H^{1}(\Omega) \mathrm{e}$ $(x, y) \in \Omega$,

$$
\Phi_{\varepsilon} \circ \tilde{F}_{\varepsilon} \circ \Phi_{\varepsilon}^{-1}(u)(x, y)=f(u(x, y)),
$$

e o lema segue observando-se a hipótese (HC*).

Agora o problema $\left(P_{\varepsilon}\right)$ pode ser formulado como uma equação abstrata

$$
\begin{cases}\dot{u}+A_{\varepsilon} u & =F_{\varepsilon}(u), \quad t>0 \\ u(0) & =u_{0}\end{cases}
$$

no espaço de fase $X_{\varepsilon}^{\frac{1}{2}}$, onde $A_{\varepsilon}: D\left(A_{\varepsilon}\right) \subset X_{\varepsilon}^{0} \rightarrow X_{\varepsilon}^{0}$ é o operador linear definido por

$$
\begin{aligned}
D\left(A_{\varepsilon}\right) & =\left\{u \in H_{\varepsilon}^{1}(\Omega):-\Delta_{x} u-\frac{1}{\varepsilon^{2}} \Delta_{y} u+u \in L^{2}(\Omega), \frac{\partial u}{\partial \eta_{x}}+\frac{1}{\varepsilon^{2}} \frac{\partial u}{\partial \eta_{y}}=0 \text { em } \partial \Omega\right\} \\
A_{\varepsilon} u & =-\Delta_{x} u-\frac{1}{\varepsilon^{2}} \Delta_{y} u+u, u \in D\left(A_{\varepsilon}\right),
\end{aligned}
$$

e $F_{\varepsilon}: X_{\varepsilon}^{\frac{1}{2}} \rightarrow X_{\varepsilon}^{0}$ é o operador de Nemitsküi associado a $f$.

Teorema 3.7. Suponhamos que a hipótese $H C^{*}$ esteja satisfeita, então para qualquer $u_{0} \in L^{2}(\Omega)$, existe uma única solução $u_{\varepsilon} \in C\left([0, \infty), L^{2}(\Omega)\right) \cap C\left((0, \infty), H_{\mathcal{\varepsilon}}^{1}(\Omega)\right)$ da equação

$$
\begin{cases}\dot{u}+A_{\varepsilon} u & =F_{\varepsilon}(u) \\ u(0) & =u_{0}\end{cases}
$$

Além disso, se o dado inicial $u_{0} \in H_{\mathcal{\varepsilon}}^{1}(\Omega)$, então a solução $u_{\varepsilon} \in C\left([0, \infty), H_{\varepsilon}^{1}(\Omega)\right)$. 
De maneira análoga o caso limite também pode ser formulado como uma equação abstrata

$$
\begin{cases}\dot{u}+A_{0} u & =F_{0}(u), \quad t>0 \\ u(0) & =u_{0}\end{cases}
$$

no espaço de fase $X_{0}^{\frac{1}{2}}$, onde $A_{0}: D\left(A_{0}\right) \subset X_{0}^{0} \rightarrow X_{0}^{0}$ é o operador linear definido por

$$
\begin{aligned}
D\left(A_{0}\right) & =\left\{u \in H_{s}^{1}(\Omega):-\Delta u+u \in L_{s}^{2}(\Omega), \frac{\partial u}{\partial \eta}=0 \text { em } \partial \Omega\right\} \\
A_{0} u & =-\Delta u+u, u \in D\left(A_{0}\right)
\end{aligned}
$$

e $F_{0}: X_{0}^{\frac{1}{2}} \rightarrow X_{0}^{0}$ é o operador de Nemitskĭi associado a $f$.

Teorema 3.8. Suponhamos que a hipótese $\left(H C^{*}\right)$ esteja satisfeita, então para qualquer $u_{0} \in$ $L_{s}^{2}(\Omega)$, existe uma única solução $u_{0} \in C\left([0, \infty), L_{s}^{2}(\Omega)\right) \cap C\left((0, \infty), H_{s}^{1}(\Omega)\right)$ da equação

$$
\begin{cases}\dot{u}+A_{0} u & =F_{0}(u) \\ u(0) & =u_{0}\end{cases}
$$

Além disso, se o dado inicial $u_{0} \in H_{s}^{1}(\Omega)$, então a solução $u_{\varepsilon} \in C\left([0, \infty), H_{s}^{1}(\Omega)\right)$.

Teorema 3.9. A familia de operadores $\left\{A_{\varepsilon}^{-1} \in \mathscr{L}\left(L^{2}(\Omega)\right)\right\}_{\varepsilon \in(0,1]}$ converge compactamente para o operador $A_{0}^{-1} \in \mathscr{L}\left(L_{s}^{2}(\Omega)\right){ }^{1}$

Demonstração: Seja $\left\{f_{\varepsilon}\right\}_{\varepsilon \in(0,1]}$ uma família uniformemente limitada, isto é, $\left\|f_{\varepsilon}\right\|_{X_{\varepsilon}^{0}}=1$, colocando $u_{\varepsilon}=A_{\varepsilon}^{-1} f_{\varepsilon}$ vemos que

$$
\int_{\Omega} \nabla_{x} u_{\varepsilon} \cdot \nabla_{x} v+\frac{1}{\varepsilon^{2}} \nabla_{y} u_{\varepsilon} \cdot \nabla_{y} v+u_{\varepsilon} v=\int_{\Omega} f_{\varepsilon} v, \quad \forall v \in X_{\varepsilon}^{\frac{1}{2}}
$$

em particular, para $v=u_{\varepsilon}, \int_{\Omega}\left|\nabla_{x} u_{\varepsilon}\right|^{2}+\frac{1}{\varepsilon^{2}}\left|\nabla_{y} u_{\varepsilon}\right|^{2}+\left|u_{\varepsilon}\right|^{2}=\int_{\Omega} f_{\varepsilon} u_{\varepsilon}$

Logo

$$
\left\|u_{\varepsilon}\right\|_{H^{1}(\Omega)}^{2} \leqslant\left\|u_{\mathcal{\varepsilon}}\right\|_{\varepsilon}^{2} \leqslant\left\|f_{\varepsilon}\right\|_{X_{\varepsilon}^{0}}\left\|u_{\varepsilon}\right\|_{H^{1}(\Omega)}
$$

e portanto $\left\|u_{\varepsilon}\right\|_{H^{1}(\Omega)} \leqslant 1$.

Portanto, a menos de subseqüências, existe $u_{0} \in H^{1}(\Omega)$ tal que $u_{\varepsilon} \rightarrow u_{0}$, fracamente em $H^{1}(\Omega)$ e fortemente em $L^{2}(\Omega)$.

Além disso

$$
\left\|u_{\varepsilon}\right\|_{H^{1}(\Omega)} \leqslant 1 \Rightarrow\left\|u_{\varepsilon}\right\|_{\varepsilon}^{2} \leqslant 1 \Rightarrow \frac{1}{\varepsilon^{2}} \int_{\Omega}\left|\nabla_{y} u_{\varepsilon}\right|^{2} \leqslant 1
$$

\footnotetext{
${ }^{1}$ Observemos que para este tipo de perturbação as extensões $E_{\varepsilon}$ coincidem com o operador identidade de $L^{2}(\Omega)$.
} 
mostrando que $\nabla_{y} u_{\varepsilon} \stackrel{\left(L^{2}(\Omega)\right)^{N}}{\longrightarrow} 0$.

Agora se $\phi \in C_{0}^{\infty}(\Omega), i=1, \cdots, N$

$$
\int_{\Omega} u_{0} \phi_{y_{i}} \leqslant\left\|u_{0}-u_{\varepsilon}\right\|_{L^{2}(\Omega)}\left\|\phi_{y_{i}}\right\|_{L^{2}(\Omega)}+\left\|\nabla_{y} u_{\varepsilon}\right\|_{\left(L^{2}(\Omega)\right)^{N}}\|\phi\|_{L^{2}(\Omega)} \stackrel{\varepsilon \rightarrow 0}{\longrightarrow} 0
$$

e então concluímos que $u_{0} \in X_{0}^{\frac{1}{2}}$. Ou seja, $A_{\varepsilon}^{-1} f_{\varepsilon} \stackrel{E}{\longrightarrow} u_{0} \in X_{0}^{\frac{1}{2}} \subset X_{0}^{0}$.

Agora consideremos $f_{\varepsilon} \stackrel{E}{\longrightarrow} f_{0}$. Logo $\left\{f_{\varepsilon}\right\}$ é uniformemente limitada. Novamente se $A_{\varepsilon}^{-1} f_{\varepsilon}=u_{\varepsilon}$ existe $u_{0} \in X_{0}^{\frac{1}{2}}$ tal que $u_{\varepsilon} \stackrel{E}{\longrightarrow} u_{0}$.

Mostremos que $u_{0}=A_{0}^{-1} f_{0}$, isto é, mostremos que $\int_{\Omega} \nabla u_{0} \cdot \nabla v+u_{0} v=\int_{\Omega} f_{0} v \quad \forall v \in X_{0}^{\frac{1}{2}}$.

Já que $f_{\varepsilon} \stackrel{E}{\longrightarrow} f_{0}$ e $u_{\varepsilon} \stackrel{E}{\longrightarrow} u_{0}$ temos $\int_{\Omega} f_{\varepsilon} v \rightarrow \int_{\Omega} f_{0} v$ e $\int_{\Omega} u_{\varepsilon} v \rightarrow \int_{\Omega} u_{0} v \quad \forall v \in X_{0}^{\frac{1}{2}}$. Além disso, da convergência $u_{\varepsilon} \stackrel{H^{1}(\Omega)}{\longrightarrow} u_{0}$, segue que $\int_{\Omega} \nabla_{x} u_{\varepsilon} \cdot \nabla_{x} v \rightarrow \int_{\Omega} \nabla u_{0} \cdot \nabla v, \quad \forall v \in X_{0}^{\frac{1}{2}}$.

Observando que $\int_{\Omega} \nabla_{x} u_{\varepsilon} \cdot \nabla_{x} v+u_{\varepsilon} v=\int_{\Omega} f_{\varepsilon} v, \quad \forall v \in X_{0}^{\frac{1}{2}}$ o resultado está provado.

Definição 3.10. Uma medida de não-compacidade $\beta$ em um espaço métrico completo $Y$ é uma função $\beta: 2^{Y} \rightarrow \mathbb{R}^{+}$satisfazendo as seguintes propriedades:

(i) $\beta(A)=0$ se, e somente se, A é pré-compacto.

(ii) $\beta(A \cup B)=\max \{\beta(A), \beta(B)\}$.

(iii) $\beta(A+B) \leqslant \beta(A)+\beta(B)$.

Lema 3.11. Para todo $0<\theta \leqslant 1$, a família $\left\{A_{\varepsilon}^{-\theta}\right\}_{\varepsilon \in(0,1]}$ converge compactamente para o operador $A_{0}^{-\theta}$.

Demonstração: Sejam $\Sigma_{-\lambda, \phi}$ como na Proposição 3.3 e $\Gamma$ a fronteira de $\Sigma_{-\lambda, \phi}$. Como

$$
A_{\varepsilon}^{-\theta}=\frac{1}{2 \pi i} \int_{\Gamma} \mu^{-\theta}\left(\mu+A_{\varepsilon}\right)^{-1} d \mu
$$

segue que a integral acima é absolutamente uniformemente convergente para $\varepsilon \in\left(0, \varepsilon_{\lambda}\right]$. Logo, dado $\eta>0$, podemos dividir o contorno $\Gamma=\Gamma_{1}^{\eta} \cup \Gamma_{2}^{\eta}$ de tal maneira que $\Gamma_{1}^{\eta}$ seja limitada e a integral

$$
\frac{1}{2 \pi i} \int_{\Gamma_{2}^{\eta}}\left\|\mu^{-\theta}\left(\mu+A_{\varepsilon}\right)^{-1}\right\|_{\mathscr{L}\left(X_{\varepsilon}\right)} d \mu \leqslant \eta, \quad \text { para todo } \varepsilon \in\left(0, \varepsilon_{\lambda}\right] .
$$

Sobre $\Gamma_{1}^{\eta}$, reescrevemos a integral como

$$
B_{\varepsilon}:=\frac{A_{\varepsilon}^{-1}}{2 \pi i} \int_{\Gamma_{1}^{\eta}} \mu^{-\theta} A_{\varepsilon}\left(\mu+A_{\varepsilon}\right)^{-1} d \mu
$$


e usamos que $\left\|\mu^{-\theta} A_{\varepsilon}\left(\mu+A_{\varepsilon}\right)^{-1}\right\|_{X_{\varepsilon}}=\left\|\mu^{-\theta}\left(I+\mu A_{\varepsilon}{ }^{-1}\right)^{-1}\right\|_{X_{\varepsilon}}$ é uniformemente limitado para $\varepsilon \in\left(0, \varepsilon_{\lambda}\right]$ e também que $\left\{A_{\varepsilon}^{-1}: \varepsilon \in\left(0, \varepsilon_{\lambda}\right]\right\}$ é compactamente convergente para garantir que $\left\{B_{\varepsilon}: \varepsilon \in\left(0, \varepsilon_{\lambda}\right]\right\}$ é também compactamente convergente. Seja $\beta: 2^{X} \rightarrow \mathbb{R}^{+}$uma medida de nãocompacidade. Agora, tomando quaisquer seqüências $\varepsilon_{n} \rightarrow 0$ e $\left\{u_{n}\right\}, u_{n} \in X,\left\|u_{n}\right\|_{X}=1$, obtemos

$$
\beta\left(\left\{A_{\varepsilon_{n}}^{-\theta} u_{n}\right\}\right) \leqslant \beta\left(B_{\varepsilon_{n}}\left\{u_{n}\right\}\right)+\beta\left(\frac{1}{2 \pi i} \int_{\Gamma_{2}^{\eta}} \mu^{-\theta}\left(\mu+A_{\varepsilon_{n}}\right)^{-1} d \mu\left\{u_{n}\right\}\right) \leqslant \eta
$$

Logo, dadas seqüências $\varepsilon_{n} \rightarrow 0$ e $\left\{u_{n}\right\}$, com $u_{n} \in X_{\mathcal{\varepsilon}},\left\|u_{n}\right\|_{X_{\varepsilon}}=1$, temos que $\beta\left(\left\{A_{\varepsilon_{n}}^{-\theta} u_{n}\right\}\right)=0$ e que $\left\{A_{\varepsilon}^{-\theta}: \varepsilon \in\left(0, \varepsilon_{\lambda}\right]\right\}$ é E-relativamente compacto.

Agora do Teorema da Convergência Dominada e do Lema 2.7, temos

$$
A_{\varepsilon}^{-\theta}=\frac{1}{2 \pi i} \int_{\Gamma}\left\|-\mu^{-\theta}\left(\mu+A_{\varepsilon}\right)^{-1}\right\|_{\mathscr{L}\left(X_{\varepsilon}\right)} d \mu \stackrel{\varepsilon \rightarrow 0}{\longrightarrow} \frac{1}{2 \pi i} \int_{\Gamma}\left\|\mu^{-\theta}\left(\mu+A_{0}\right)^{-1}\right\|_{\mathscr{L}\left(X_{\varepsilon}\right)} d \mu=A_{0}^{-\theta},
$$

para todo $\mu \in \Gamma$.

Uma vez fixados $X_{\varepsilon}^{0}=L^{2}(\Omega)$ e $X_{\varepsilon}^{1}=D\left(A_{\varepsilon}\right)$ (resp. $X_{0}^{0}=L_{s}^{2}(\Omega)$ e $X_{0}^{1}=D\left(A_{0}\right)$ ), consideremos $\left\{\left(X_{\varepsilon}^{\alpha}, A_{\varepsilon}^{\alpha}\right)\right\}_{\alpha}$ a escala de interpolação-extrapolação gerada pelo par $\left(X_{\varepsilon}^{0}, A_{\varepsilon}\right)$ (resp. $\left(X_{0}^{0}, A_{0}\right)$, (que coincide com a escala de espaços de potência fracionária do operador $A_{\varepsilon}$ ). Abusando de nossa notação, a partir de agora identificaremos todas as realizações do operador $A_{\varepsilon}$ (resp. $A_{0}$ ) nesta escala, e as denotaremos também por $A_{\varepsilon}$ (resp. $\left.A_{0}\right)$.

Mostramos acima que se $\left\{A_{\mathcal{\varepsilon}}^{-1} f_{\varepsilon}\right\}$ é uma seqüencia uniformemente limitada, então esta seqüencia possui um limite fraco em $H^{1}(\Omega)$ que vive em $H_{s}^{1}$. Entretanto, podemos mostrar na verdade, que esta convergência se dá na norma $H_{\mathcal{\varepsilon}}^{1}(\Omega)$.

Lema 3.12. Sejam $\varepsilon_{n} \stackrel{n \rightarrow \infty}{\longrightarrow} 0$ e $\left\{f_{\varepsilon_{n}}\right\}, f_{\varepsilon_{n}} \in X_{\varepsilon_{n}}^{0}$, e $f_{0} \in X_{0}^{0}$ com $f_{\varepsilon_{n}} \stackrel{E}{\longrightarrow} f_{0}$. Então se $u_{\varepsilon_{n}}=A_{\varepsilon_{n}}^{-1} f_{\varepsilon_{n}}$ e $u_{0}=A_{0}^{-1} f_{0}$

$$
\lim _{n \rightarrow \infty}\left\|u_{\varepsilon_{n}}-u_{0}\right\|_{H^{1}(\Omega)}=0
$$

Demonstração: Tomando $v \in X_{0}^{\frac{1}{2}}$ e passando ao limite a expressão

$$
\int_{\Omega} \nabla_{x} u_{\varepsilon_{n}} \cdot \nabla_{x} v+\frac{1}{\varepsilon_{n}^{2}} \nabla_{y} u_{\varepsilon_{n}} \cdot \nabla_{y} v+u_{\varepsilon_{n}} v=\int_{\Omega} f_{\varepsilon_{n}} v
$$

obtemos $\int_{\Omega} \nabla_{x} \bar{u} \cdot \nabla_{x} v+\bar{u} v=\int_{\Omega} f_{0} v$. Como $v \in X_{0}^{\frac{1}{2}}$ é arbitrário, temos $\bar{u}=A^{-1} f_{0}=u_{0}$. 
Por outro lado, a convergência fraca $u_{\varepsilon_{n}} \stackrel{H^{1}(\Omega)}{\longrightarrow} u_{0}$ implica,

$$
\begin{aligned}
\left\|u_{0}\right\|_{H^{1}(\Omega)}^{2}=\int_{\Omega}\left|\nabla_{x} u_{0}\right|^{2}+\left|u_{0}\right|^{2} & \leqslant \lim _{n \rightarrow \infty}\left(\int_{\Omega}\left|\nabla_{x} u_{\varepsilon_{n}}\right|^{2}+\left|\nabla_{y} u_{\varepsilon_{n}}\right|^{2}+\left|u_{\varepsilon_{n}}\right|^{2}\right) \\
& \leqslant \lim _{n \rightarrow \infty}\left(\int_{\Omega}\left|\nabla_{x} u_{\varepsilon_{n}}\right|^{2}+\left|\nabla_{y} u_{\varepsilon_{n}}\right|^{2}+\left|u_{\varepsilon_{n}}\right|^{2}\right) \\
& \leqslant \lim _{n \rightarrow \infty}\left(\int_{\Omega}\left|\nabla_{x} u_{\varepsilon_{n}}\right|^{2}+\frac{1}{\varepsilon^{2}}\left|\nabla_{y} u_{\varepsilon_{n}}\right|^{2}+\left|u_{\varepsilon_{n}}\right|^{2}\right) \\
& =\lim _{n \rightarrow \infty} \int_{\Omega} f_{\varepsilon_{n}} u_{\varepsilon_{n}}=\int_{\Omega} f_{0} u_{0}=\int_{\Omega}\left|\nabla_{x} u_{0}\right|^{2}+\left|u_{0}\right|^{2}=\left\|u_{0}\right\|_{H^{1}(\Omega)}^{2}
\end{aligned}
$$

Mostrando que $\lim _{n \rightarrow \infty}\left\|u_{\varepsilon_{n}}\right\|_{H^{1}(\Omega)}=\left\|u_{0}\right\|_{H^{1}(\Omega)}$.

Lema 3.13. Com as hipóteses do Lema anterior, dada a caracterização variacional das soluções $u_{\varepsilon}$ e $u_{0}$ dos problemas $A_{\varepsilon} u=f_{\varepsilon}$ e $A_{0} u=f_{0}$ respectivamente, sejam $\lambda_{\varepsilon}$ e $\lambda_{0}$ tais que

$$
\begin{aligned}
& \lambda_{\varepsilon}=\frac{1}{2}\left\|u_{\varepsilon}\right\|_{\varepsilon}^{2}-\int_{\Omega} f_{\varepsilon} u_{\varepsilon}=\min _{\phi \in X_{\varepsilon}^{\frac{1}{2}}}\left(\frac{1}{2}\|\phi\|_{\varepsilon}^{2}-\int_{\Omega} f_{\varepsilon} \phi\right) \\
& \lambda_{0}=\frac{1}{2}\left\|u_{0}\right\|_{X_{0}^{\frac{1}{2}}}^{2}-\int_{\Omega} f_{0} u_{0}=\min _{\phi \in X_{0}^{\frac{1}{2}}}\left(\frac{1}{2}\|\phi\|_{X_{0}^{\frac{1}{2}}}^{2}-\int_{\Omega} f_{0} \phi\right) .
\end{aligned}
$$

Então

$$
\lim _{\varepsilon \rightarrow 0} \lambda_{\varepsilon}=\lambda_{0}
$$

Demonstração: Primeiramente observemos que

$$
\lambda_{\varepsilon} \leqslant \frac{1}{2}\left\|u_{0}\right\|_{X_{0}^{\frac{1}{2}}}^{2}-\int_{\Omega} f_{\varepsilon} u_{0}=\frac{1}{2}\left\|u_{0}\right\|_{X_{0}^{\frac{1}{2}}}^{2}-\int_{\Omega} f_{0} u_{0}-\int_{\Omega}\left(f_{\varepsilon}-f_{0}\right) u_{0}=\lambda_{0}-\int_{\Omega}\left(f_{\varepsilon}-f_{0}\right) u_{0} .
$$

Logo

$$
\limsup _{\varepsilon \rightarrow 0} \lambda_{\varepsilon} \leqslant \lambda_{0}
$$

Por outro lado,

$$
\begin{aligned}
\lambda_{0}= & \frac{1}{2} \int_{\Omega}\left(\left|\nabla u_{0}\right|^{2}+\left|u_{0}\right|^{2}\right)-\int_{\Omega} f_{0} u_{0} \\
= & \frac{1}{2} \int_{\Omega}\left(\left|\nabla_{x} u_{0}-\nabla_{x} u_{\varepsilon}+\nabla_{x} u_{\varepsilon}\right|^{2}+\left|u_{0}-u_{\varepsilon}+u_{\varepsilon}\right|^{2}\right)-\int_{\Omega} f_{0}\left(u_{0}-u_{\varepsilon}\right)-\int_{\Omega}\left(f_{0}-f_{\varepsilon}\right) u_{\varepsilon}-\int_{\Omega} f_{\varepsilon} u_{\varepsilon} \\
= & \frac{1}{2} \int_{\Omega}\left(\left|\nabla_{x} u_{0}-\nabla_{x} u_{\varepsilon}\right|^{2}+\left|u_{0}-u_{\varepsilon}\right|^{2}\right)+\int_{\Omega}\left(\nabla_{x} u_{0}-\nabla_{x} u_{\varepsilon}\right) \cdot \nabla_{x} u_{\varepsilon}+\frac{1}{2} \int_{\Omega}\left|\nabla_{x} u_{\varepsilon}\right|^{2}+\int_{\Omega}\left(u_{0}-u_{\varepsilon}\right) u_{\varepsilon} \\
& +\frac{1}{2} \int_{\Omega}\left|u_{\varepsilon}\right|^{2}-\int_{\Omega} f_{0}\left(u_{0}-u_{\varepsilon}\right)-\int_{\Omega}\left(f_{0}-f_{\varepsilon}\right) u_{\varepsilon}-\int_{\Omega} f_{\varepsilon} u_{\varepsilon} \\
\leqslant & \frac{1}{2}\left\|u_{0}-u_{\varepsilon}\right\|_{H^{1}(\Omega)}^{2}+\lambda_{\varepsilon}+\int_{\Omega}\left(\nabla_{x} u_{0}-\nabla_{x} u_{\varepsilon}\right) \cdot \nabla_{x} u_{\varepsilon}+\int_{\Omega}\left(u_{0}-u_{\varepsilon}\right) u_{\varepsilon}-\int_{\Omega} f_{0}\left(u_{0}-u_{\varepsilon}\right)-\int_{\Omega}\left(f_{0}-f_{\varepsilon}\right) u_{\varepsilon}
\end{aligned}
$$

E o resultado segue de (6.6) e do Lema anterior. 
Lema 3.14. Sejam $\varepsilon_{n} \stackrel{n \rightarrow \infty}{\longrightarrow} 0$ e $\left\{f_{\varepsilon_{n}}\right\}, f_{\varepsilon_{n}} \in X_{\varepsilon_{n}}^{0}$, e $f_{0} \in X_{0}^{0}$ com $f_{\varepsilon_{n}} \stackrel{E}{\longrightarrow} f_{0}$. Então se $u_{\varepsilon_{n}}=A_{\varepsilon_{n}}^{-1} f_{\varepsilon_{n}}$ e $u_{0}=A_{0}^{-1} f_{0}$

$$
\lim _{n \rightarrow \infty}\left\|u_{\varepsilon_{n}}-u_{0}\right\|_{\varepsilon_{n}}=0
$$

Demonstração: De maneira análoga a prova do Lema anterior temos

$$
\begin{aligned}
\lambda_{\varepsilon} & =\frac{1}{2}\left\|u_{\varepsilon}\right\|_{\varepsilon}^{2}-\int_{\Omega} f_{\varepsilon} u_{\varepsilon} \\
& =\frac{1}{2}\left\|u_{\varepsilon}-u_{0}+u_{0}\right\|_{\varepsilon}^{2}-\int_{\Omega} f_{\varepsilon} u_{\varepsilon}-\int_{\Omega} f_{\varepsilon}\left(u_{\varepsilon}-u_{0}\right)-\int_{\Omega}\left(f_{\varepsilon}-f_{0}\right) u_{0}-\int_{\Omega} f_{0} u_{0} \\
& =\frac{1}{2}\left\|u_{\varepsilon}-u_{0}\right\|_{\varepsilon}^{2}+\frac{1}{\varepsilon^{2}}\left|\nabla_{y} u_{\varepsilon}\right|^{2}+\frac{1}{2}\left\|u_{0}\right\|_{X_{0}^{\frac{1}{2}}}^{2}-\int_{\Omega} f_{0} u_{0} \\
& +\int_{\Omega}\left(\nabla_{x} u_{\varepsilon}-\nabla_{x} u_{0}\right) \cdot \nabla_{x} u_{0}+\int_{\Omega}\left(u_{\varepsilon}-u_{0}\right) u_{0}-\int_{\Omega} f_{\varepsilon}\left(u_{\varepsilon}-u_{0}\right)-\int_{\Omega}\left(f_{\varepsilon}-f_{0}\right) u_{0} \\
& =\frac{1}{2}\left\|u_{0}-u_{\varepsilon}\right\|_{\varepsilon}^{2}+\lambda_{0} \\
& +\int_{\Omega}\left(\nabla_{x} u_{0}-\nabla_{x} u_{\varepsilon}\right) \cdot \nabla_{x} u_{\varepsilon}+\int_{\Omega}\left(u_{0}-u_{\varepsilon}\right) u_{\varepsilon}-\int_{\Omega} f_{\varepsilon}\left(u_{\varepsilon}-u_{0}\right)-\int_{\Omega}\left(f_{\varepsilon}-f_{0}\right) u_{0},
\end{aligned}
$$

e o resultado segue dos lemas anteriores.

Corolário 3.15. A família de operadores $\left\{A_{\varepsilon}^{-1} \in \mathscr{L}\left(X_{\varepsilon}^{\frac{1}{2}}\right)\right\}_{\varepsilon \in(0,1]}$ converge compactamente para o operador $A_{0}^{-1} \in \mathscr{L}\left(X_{0}^{\frac{1}{2}}\right)$.

A seguir verificamos as hipóteses necessárias aos resultados abstratos do Capítulo 2.

Lema 3.16. A família de operadores $\left\{A_{\varepsilon}^{-1} F_{\varepsilon}\right\}_{\varepsilon \in(0,1]}$ converge compactamente para o operador $A_{0}^{-1} F_{0}$.

Demonstração: Da limitação uniforme das $F_{\varepsilon}$ é fácil ver que se $\left\{u_{\varepsilon}\right\}, u_{\varepsilon} \in X_{\varepsilon}$ é uma família uniformemente limitada então $\left\{F_{\varepsilon}\left(u_{\varepsilon}\right)\right\}$ é também uniformemente limitada e como $A_{\varepsilon}^{-1} \stackrel{C C}{\longrightarrow} A_{0}^{-1}$ a família $\left\{A_{\varepsilon}^{-1} F_{\varepsilon}\left(u_{\varepsilon}\right)\right\}$ é $E$-relativamente compacta. Ainda se $u_{\varepsilon} \stackrel{E}{\longrightarrow} u_{0}$ da continuidade da $f$ temos que $F_{\varepsilon}\left(u_{\varepsilon}\right) \stackrel{E}{\longrightarrow} F_{0}\left(u_{0}\right)$. Portanto $A_{\varepsilon}^{-1} F_{\varepsilon}\left(u_{\varepsilon}\right) \stackrel{E}{\longrightarrow} A_{0}^{-1} F_{0}\left(u_{0}\right)$ uma vez que $A_{\varepsilon}^{-1} \stackrel{E E}{\longrightarrow} A_{0}^{-1}$.

Lema 3.17. Dada uma seqüencia $\varepsilon_{n} \stackrel{n \rightarrow \infty}{\longrightarrow} 0$, considere uma seqüencia $\left\{u_{\varepsilon_{n}}\right\}, u_{\varepsilon_{n}} \in X_{\varepsilon_{n}}^{\frac{1}{2}}$ com $\left\|u_{\varepsilon_{n}}-E_{\varepsilon_{n}} u_{0}\right\|_{X_{\varepsilon_{n}}^{\frac{1}{2}}} \rightarrow 0, \quad n \rightarrow \infty$. Consideremos a familia de operadores

$$
F_{\varepsilon_{n}}^{\prime}\left(u_{\varepsilon_{n}}\right): X_{\varepsilon_{n}}^{\frac{1}{2}} \longrightarrow X_{\varepsilon_{n}}^{0}, \quad \varepsilon \in[0,1]
$$

Se $\left\{v_{\varepsilon_{n}}\right\}$ é uma seqüencia, $X_{\varepsilon_{n}}^{\frac{1}{2}} \ni v_{\mathcal{E}_{n}} \stackrel{X_{\varepsilon_{n}}}{\longrightarrow} v_{0} \in X_{0}^{\frac{1}{2}}$, então $F_{\varepsilon_{n}}^{\prime}\left(u_{\varepsilon_{n}}\right) v_{\varepsilon_{n}} \stackrel{X_{\varepsilon_{n}}}{\longrightarrow} F_{0}^{\prime}\left(u_{0}\right) v_{0}$. 
Demonstração: Observemos que,

$$
\begin{aligned}
\left\|F_{\varepsilon_{n}}^{\prime}\left(u_{\varepsilon_{n}}\right) v_{\varepsilon_{n}}-E_{\varepsilon_{n}} F_{0}^{\prime}\left(u_{0}\right) v_{0}\right\|_{X_{\varepsilon_{n}}} & \\
& \leqslant\left\|F_{\varepsilon_{n}}^{\prime}\left(u_{\varepsilon_{n}}\right) v_{\varepsilon_{n}}-F_{\varepsilon_{n}}^{\prime}\left(u_{\varepsilon_{n}}\right) E_{\varepsilon_{n}} v_{0}\right\|_{X_{\varepsilon_{n}}}+\left\|F_{\varepsilon_{n}}^{\prime}\left(u_{\varepsilon_{n}}\right) E_{\varepsilon_{n}} v_{0}-E_{\varepsilon_{n}} F_{0}^{\prime}\left(u_{0}\right) v_{0}\right\|_{X_{\varepsilon_{n}}} \\
& \leqslant\left\|F_{\varepsilon_{n}}^{\prime}\left(u_{\varepsilon_{n}}\right)\right\|_{\left.\mathscr{L}_{\left(X_{n}\right.} \frac{1}{2}, X_{\varepsilon_{n}}\right)}\left\|v_{\varepsilon_{n}}-E_{\varepsilon_{n}} v_{0}\right\|_{X_{\varepsilon_{n}}}+\left\|F_{\varepsilon_{n}}^{\prime}\left(u_{\varepsilon_{n}}\right) E_{\varepsilon_{n}} v_{0}-F_{\varepsilon_{n}}^{\prime}\left(E_{\varepsilon_{n}} u_{0}\right) E_{\varepsilon_{n}} v_{0}\right\|_{X_{\varepsilon_{n}}} \\
& +\left\|F_{\varepsilon_{n}}^{\prime}\left(E_{\varepsilon_{n}} u_{0}\right) E_{\varepsilon_{n}} v_{0}-F_{0}^{\prime}\left(u_{0}\right) v_{0}\right\|_{X_{\varepsilon_{n}}} .
\end{aligned}
$$

Logo o resultado segue da $C^{1}$-regularidade de $F_{\varepsilon_{n}}^{\prime}$, da Lipschitz continuidade de $f$ que implica que $\sup _{\varepsilon \in[0,1]}\left\|F_{\varepsilon}^{\prime}\left(u_{\varepsilon}\right)\right\|_{\mathscr{L}\left(X_{\varepsilon}^{\frac{1}{2}}, X_{\varepsilon}\right)}<\infty$ e da identidade $F_{\varepsilon}^{\prime}\left(E_{\varepsilon_{n}} u_{0}\right) E_{\varepsilon_{n}} v_{0}=F_{0}^{\prime}\left(u_{0}\right) v_{0}, \forall \varepsilon \in[0,1]$.

Corolário 3.18. Se $\left\{u_{\varepsilon}^{*}\right\}, u_{\varepsilon}^{*} \in \mathscr{E}_{\varepsilon}$ é uma seqüencia de equilíbrios tais que $u_{\varepsilon}^{*} \stackrel{E}{\longrightarrow} u_{0}^{*} \in \mathscr{E}_{0}$. Então a familia de operadores $\left\{A_{\varepsilon}^{-1} F_{\varepsilon}^{\prime}\left(u_{\varepsilon}^{*}\right)\right\}_{\varepsilon \in(0,1]}$ converge compactamente para o operador $A_{0}^{-1} F_{0}^{\prime}\left(u_{0}^{*}\right)$.

Considerando os funcionais $V_{\varepsilon}: X_{\varepsilon}^{\frac{1}{2}} \rightarrow \mathbb{R}$, (resp. $V_{0}: X_{0}^{\frac{1}{2}} \rightarrow \mathbb{R}$ ) dados por

$$
\begin{gathered}
V_{\varepsilon}(\varphi)=\frac{1}{2} \int_{\Omega}\left(\left|\nabla_{x} \varphi\right|^{2}+\frac{1}{\varepsilon^{2}}\left|\nabla_{y} \varphi\right|^{2}+\varphi^{2}\right) d x-\int_{\Omega} F(\varphi) d x, \\
V_{0}(\varphi)=\frac{1}{2} \int_{\Omega}\left(|\nabla \varphi|^{2}+\varphi^{2}\right) d x-\int_{\Omega} F(\varphi) d x,
\end{gathered}
$$

onde $F$ é uma primitiva de $f$, vemos que os problemas (3.2) e (3.5) têm estrutura gradiente.

Teorema 3.19. Com a notação introduzida acima, são válidas as seguintes afirmações

(i) Sejam $\lambda_{\varepsilon, 1} \leqslant \lambda_{\varepsilon, 2} \leqslant \cdots$ os autovalores de $A_{\varepsilon}$ contados com suas respectivas multiplicidades. Então existe uma subseqüência $\left\{\lambda_{\varepsilon_{n}, i}\right\}$ tal que $\lambda_{\varepsilon_{n}, i} \rightarrow \lambda_{0, i}$. Reciprocamente se $\lambda_{0, i}$ é um autovalor de $A_{0}$ então existe uma subseqüência $\left\{\lambda_{\varepsilon_{n}, i}\right\}$ tal que $\lambda_{\varepsilon_{n}, i} \rightarrow \lambda_{0, i}$.

(ii) $S e \varphi_{\varepsilon, i}$ é a auto-função associada ao respectivo autovalor $\lambda_{\varepsilon, i}$ então existe uma subseqüência $\left\{\varphi_{\varepsilon_{n}, i}\right\}$ tal que $\varphi_{\varepsilon_{n}, i} \stackrel{H_{\varepsilon}^{1}(\Omega)}{\longrightarrow} \varphi_{0, i}$. Reciprocamente se $\varphi_{0, i}$ é um autovalor de $A_{0}$ então existe uma subseqüência $\left\{\varphi_{\varepsilon_{n}, i}\right\}$ tal que $\varphi_{\varepsilon_{n}, i} \stackrel{H_{\varepsilon}^{1}(\Omega)}{\longrightarrow} \varphi_{0, i}$.

(iii) $S e\left\{\mathscr{A}_{\varepsilon} \subset H^{1}(\Omega)_{\varepsilon}\right\}$ é a família de atratores para o semifluxo gerado pela família de equações (3.2) então $\left\{\mathscr{A}_{\varepsilon}\right\}$ é contínua em $\varepsilon=0$. 



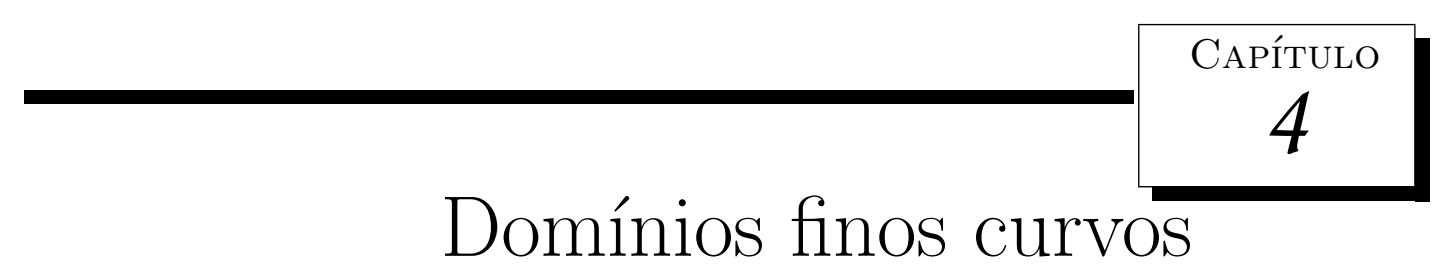

Aqui o exemplo anterior é generalizado nos seguinte sentido. Consideramos um modelo de reação-difusão definido em um domínio $\Omega \subset \mathbb{R}^{n}, n \geqslant 2$, que se colapsa a uma subvariedade $\mathscr{M}$ imersa de dimensão $k$ de $\mathbb{R}^{n}(k<n)$. Para $p \in \mathscr{M}$ denotamos $T_{p} \mathscr{M}$ o espaço tangente a $\mathscr{M}$ no ponto $p$ e o identificamos com um subespaço vetorial de $\mathbb{R}^{n}$.

Definição 4.1. Se $\mathscr{M}$ é uma variedade de classe $C^{r}, r \geqslant 2$, um subconjunto aberto $U$ de $\mathbb{R}^{n}$ é uma vizinhança normal de $\mathscr{M}$ se existe uma aplicação $\phi: U \rightarrow \mathscr{M}$ de classe $C^{r-1}$, tal que

i) Se $x \in U$ e $p \in \mathscr{M}$ então $\phi(x)=p$ se e somente se o vetor $x-p$ é ortogonal a $T_{p} \mathscr{M}$;

ii) $\varepsilon x+(1-\varepsilon) \phi(x) \in U$ quaisquer que sejam $x \in U$ e $\varepsilon \in[0,1]$.

Observamos que pelo Teorema da vizinhança tubular, uma vizinhança normal de $\mathscr{M}$ sempre existe.

As seguintes propriedades são conseqüências imediatas da definição anterior.

Proposição 4.2. Seja $U$ uma vizinhança normal de $\mathscr{M}$. Então a aplicação $\phi$ da definição 4.1 é unicamente determinada. Além disso,

i) $\phi(U)=\mathscr{M}$ e $\phi(x)=x$ se e somente se $x \in \mathscr{M}$;

ii) $D \phi(x) v=0$ para todo $x \in U$ e todo vetor $v$ ortogonal a $T_{\phi(x)} \mathscr{M}$

Seja $(U, \phi)$ uma vizinhança normal de $\mathscr{M}$. Para $x \in U$ seja $Q(x)=\mathbb{R}^{n} \rightarrow \mathbb{R}^{n}$ a projeção ortogonal de $\mathbb{R}^{n} \cong T_{\phi(x)} \mathbb{R}^{n}$ sobre $T_{\phi(x)} \mathscr{M}$ e $P(x):=I-Q(x)$. Note que $P(x)$ é a projeção ortogonal de $\mathbb{R}^{n} \cong T_{\phi(x)} \mathbb{R}^{n}$ sobre o complemento ortogonal de $T_{\phi(x)} \mathscr{M}$ em $\mathbb{R}^{n} \cong T_{\phi(x)} \mathbb{R}^{n}$.

Para $\varepsilon \in[0,1]$ definimos a compressão na direção normal à $\mathscr{M}$,

$$
\Phi_{\varepsilon}(x):=\varepsilon x+(1-\varepsilon) \phi(x)=\phi(x)+\varepsilon(x-\phi(x)) .
$$

A seguir coletamos uma série de propriedades derivadas desta definição e que podem ser encontradas em [43]. 
Proposição 4.3. A aplicação

$$
\begin{array}{ccc}
{[0,1] \times U} & \rightarrow & \mathbb{R}^{n} \\
(\varepsilon, x) & \mapsto \Phi_{\varepsilon}(x)
\end{array}
$$

é contínua. Além disso para $\varepsilon \in(0,1]$

i) $\Phi_{\varepsilon}(U)=\left\{y \in U: \phi(y)+\frac{1}{\varepsilon}(y-\phi(y)) \in U\right\}, \Phi_{\varepsilon}(U)$ é aberto em $\mathbb{R}^{n}$ e $\Phi_{\varepsilon}: U \rightarrow \Phi_{\varepsilon}(U)$ é um difeomorfismo de classe $C^{r-1}$ com inversa

$$
\Phi_{\varepsilon}^{-1}(y)=\phi(y)+\frac{1}{\varepsilon}(y-\phi(y)), \quad y \in \Phi_{\varepsilon}(U)
$$

ii) $\phi\left(\Phi_{\varepsilon}(x)\right)=\phi(x)$ para todo $x \in U$.

Proposição 4.4. Para $x \in U$ e $\varepsilon \in[0,1]$ definimos a transformação

$$
J_{\mathcal{E}}(x)=\left\{\begin{array}{l}
\varepsilon^{-\frac{n-k}{2}}\left|\operatorname{det} D \Phi_{\varepsilon}(x)\right|, \quad \varepsilon>0, \\
\left|\operatorname{det}\left(D \phi(x)||_{\phi(x)} \mathscr{M}\right)\right|, \quad \varepsilon=0 .
\end{array}\right.
$$

Então $J_{\mathcal{\varepsilon}}(x)>0$ para todo $x \in U$ e $\varepsilon \in[0,1]$. A aplicação

$$
\begin{array}{cl}
{[0,1] \times U} & \rightarrow \mathbb{R} \\
(\varepsilon, x) & \mapsto J_{\mathcal{E}}(x)
\end{array}
$$

é contínua e para todo $x \in U$ e $\varepsilon \in[0,1]$ existe uma transformação linear $S_{\varepsilon}(x): \mathbb{R}^{n} \rightarrow \mathbb{R}^{n}$ tal que para $\varepsilon \in(0,1]$

$$
D \Phi_{\varepsilon}^{-1}\left(\Phi_{\varepsilon}(x)\right)=S_{\varepsilon}(x)+\frac{1}{\varepsilon} P(x), \quad \text { para todo } x \in U
$$

e assim,

$$
\left(D \Phi_{\varepsilon}^{-1}\left(\Phi_{\varepsilon}(x)\right)\right)^{T}=S_{\varepsilon}(x)^{T}+\frac{1}{\varepsilon} P(x), \quad \text { para todo } x \in U .
$$

Além disso,

i) a aplicação

$$
\begin{aligned}
{[0,1] \times U } & \rightarrow \mathscr{L}\left(\mathbb{R}^{n}, \mathbb{R}^{n}\right) \\
(\varepsilon, x) & \mapsto \quad S_{\varepsilon}(x) \quad\left(\operatorname{resp} .(\varepsilon, x) \mapsto S_{\varepsilon}(x)^{T}\right)
\end{aligned}
$$

é contínua;

ii) para todo $x \in U, \varepsilon \in[0,1]$ e $v \in\left(T_{\phi(x)} \mathscr{M}\right)^{\perp}$

$$
S_{\varepsilon}(x) v=S_{\varepsilon}(x)^{T} v=0
$$

iii) para todo $x \in U$ e $\varepsilon \in[0,1]$ as transformações

$$
\left.S_{\varepsilon}(x)\right|_{T_{\phi(x)} \mathscr{M}}: T_{\phi(x)} \mathscr{M} \rightarrow T_{\phi(x)} \mathscr{M}
$$


$e$

$$
\left.S_{\varepsilon}(x)^{T}\right|_{T_{\phi(x)} \mathscr{M}}: T_{\phi(x)} \mathscr{M} \rightarrow T_{\phi(x)} \mathscr{M}
$$

são bem definidas e bijetoras. Também

$$
\left(\left.S_{0}(x)\right|_{T_{\phi(x)} \mathscr{M}}\right)^{-1}=\left.D \phi(x)\right|_{T_{\phi(x)} \mathscr{M}}
$$

$e$

$$
\left(\left.S_{0}(x)^{T}\right|_{T_{\phi(x)}} \mathscr{M}\right)^{-1}=\left.D \phi(x)^{T}\right|_{T_{\phi(x)}} \mathscr{M}
$$

Finalmente, $\phi: U \rightarrow \mathscr{M}$ é uma aplicação aberta.

Seja $\Omega$ um domínio limitado e suave de $\mathbb{R}^{n}$. Supomos que $\bar{\Omega} \subset U$. Para $\varepsilon \in(0,1]$ seja $\Omega_{\varepsilon}:=\Phi_{\varepsilon}(\Omega)$ o domínio comprimido. Como nos capítulos anteriores nosso interesse é estudar o comportamento assintótico das soluções do problema

$$
\begin{array}{ll}
u_{t}=\Delta u-u+f(u), & (0, \infty) \times \Omega_{\varepsilon} \\
\frac{\partial u}{\partial \eta}=0, & (0, \infty) \times \partial \Omega_{\varepsilon}
\end{array}
$$

Para este caso também nos fazemos valer de um isomorfismo linear de $H^{1}\left(\Omega_{\varepsilon}\right)$ sobre $H^{1}(\Omega)$ (resp. $L^{2}\left(\Omega_{\varepsilon}\right)$ sobre $L^{2}(\Omega)$ ), dado por

$$
u \stackrel{T_{\varepsilon}}{\longmapsto} u \circ \Phi_{\varepsilon}
$$

Dado $\varepsilon \in(0,1]$, a formulação variacional da equação 4.2 é dada através da forma bilinear $\tilde{a}_{\varepsilon}: H^{1}\left(\Omega_{\varepsilon}\right) \times H^{1}\left(\Omega_{\varepsilon}\right)$,

$$
\tilde{a}_{\varepsilon}(u, v)=\int_{\Omega_{\varepsilon}}\langle\nabla u(x), \nabla v(x)\rangle+u(x) v(x) d x, \quad u, v \in H^{1}\left(\Omega_{\varepsilon}\right),
$$

onde $\langle\cdot, \cdot\rangle$ denota o produto interno usual em $\mathbb{R}^{n}$. Através da transformação $T_{\varepsilon}$ derivamos uma forma bilinear equivalente $a_{\varepsilon}: H^{1}(\Omega) \times H^{1}(\Omega)$, dada por

$$
\begin{aligned}
a_{\mathcal{\varepsilon}}(u, v)= & \int_{\Omega} J_{\mathcal{\varepsilon}}(x)\left\langle S_{\mathcal{\varepsilon}}(x)^{T} \nabla u(x), S_{\mathcal{\varepsilon}}(x)^{T} \nabla v(x)\right\rangle d x \\
& +\frac{1}{\varepsilon^{2}} \int_{\Omega} J_{\mathcal{E}}(x)\langle P(x) \nabla u(x), P(x) \nabla v(x)\rangle d x+\int_{\Omega} J_{\mathcal{\varepsilon}}(x) u(x) v(x) d x .
\end{aligned}
$$

Observemos que pela fórmula de mudança de variáveis e pela Proposição 4.4 temos para $\varepsilon \in(0,1]$,

$$
a_{\varepsilon}\left(u \circ \Phi_{\varepsilon}, v \circ \Phi_{\varepsilon}\right)=\varepsilon^{-\frac{n-k}{2}} \tilde{a}_{\varepsilon}(u, v)
$$

quaisquer que sejam $u, v \in H^{1}\left(\Omega_{\varepsilon}\right)$.

Observemos ainda que se $u \in H^{1}(\Omega)$,

$$
a_{\varepsilon}(u, u) \rightarrow\left\{\begin{array}{cl}
\int_{\Omega} J_{0}(x)\left(\left\langle S_{0}(x)^{T} \nabla u(x), S_{0}(x)^{T} \nabla u(x)\right\rangle+u(x)^{2}\right) d x, & \text { se } P(x) \nabla u(x)=0 \text { q.s } \\
\infty & \text { caso contrário. }
\end{array}\right.
$$


Isto nos motiva definir o espaço

$$
H_{s}^{1}(\Omega):=\left\{u \in H^{1}(\Omega): P(x) \nabla u(x)=0 \text { q.s. em } \Omega\right\},
$$

e a forma bilinear limite

$$
\begin{gathered}
a_{0}: H_{s}^{1}(\Omega) \times H_{s}^{1}(\Omega) \rightarrow \mathbb{R} \\
a_{0}(u, v)=\int_{\Omega} J_{0}(x)\left(\left\langle S_{0}(x)^{T} \nabla u(x), S_{0}(x)^{T} \nabla v(x)\right\rangle+u(x) v(x)\right) d x .
\end{gathered}
$$

Proposição 4.5. O espaço $H_{s}^{1}(\Omega)$ é um espaço de Hilbert de dimensão infinita.

Demonstração: Ver [43].

Para $\varepsilon \in[0,1]$ definimos $L_{\mathcal{\varepsilon}}^{2}(\Omega):=$ o espaço $L^{2}(\Omega)$ munido do produto interno $(u, v)_{\varepsilon}=\int_{\Omega} J_{\varepsilon}(x) u(x) v(x) d x$. Também $L_{s}^{2}(\Omega):=$ fecho de $H_{s}^{1}(\Omega)$ em $L_{0}^{2}(\Omega)$.

Pela Proposição 4.4 existem constantes positivas $c_{1}$ e $c_{2}$ independentes de $\varepsilon$ tais que para todo $u \in L^{2}(\Omega)$ e $\varepsilon \in[0,1]$

$$
c_{1}(u, u)_{\varepsilon} \leqslant\|u\|_{L^{2}(\Omega)}^{2} \leqslant c_{2}(u, u)_{\varepsilon} .
$$

Dado $\varepsilon>0$ denotamos por $H_{\mathcal{\varepsilon}}^{1}(\Omega):=$ o espaço $H^{1}(\Omega)$ munido de norma

$$
\|u\|_{\varepsilon}^{2}=a_{\varepsilon}(u, u) \text {. }
$$

Finalmente, em $H_{s}^{1}(\Omega)$ introduzimos a norma

$$
\|u\|_{0}^{2}=a_{0}(u, u)
$$

Proposição 4.6. Para todo $\delta \in(0,1)$ existe $\bar{\varepsilon} \in(0,1]$ tal que

$$
(1-\delta)(u, u)_{0} \leqslant(u, u)_{\varepsilon} \leqslant(1+\delta)(u, u)_{0}
$$

para todo $u \in L^{2}(\Omega)$ e $\varepsilon \in(0, \bar{\varepsilon}]$. Também

$$
(1-\delta) a_{0}(u, u) \leqslant a_{\varepsilon}(u, u) \leqslant(1+\delta) a_{0}(u, u)
$$

para todo $u \in H_{s}^{1}(\Omega)$ e $\varepsilon \in(0, \bar{\varepsilon}]$.

Adicionalmente, sempre que $u, v \in L^{2}(\Omega)$

$$
(u, v)_{\varepsilon} \rightarrow(u, v)_{0}, \text { quando } \varepsilon \rightarrow 0 .
$$

Além disso, em $H_{s}^{1}(\Omega)$ as normas $\|\cdot\|_{\varepsilon} e\|\cdot\|_{0}$ são equivalentes, com constante de equivalência independente de $\boldsymbol{\varepsilon}$ e

$$
a_{\mathcal{\varepsilon}}(u, u) \rightarrow a_{0}(u, u), \text { quando } \varepsilon \rightarrow 0,
$$

para todo $u \in H_{s}^{1}(\Omega)$. Finalmente existe uma constante $\eta>0$ tal que

$$
\eta\|u\|_{H^{1}(\Omega)} \leqslant\|u\|_{\varepsilon}, \text { para todo } u \in H^{1}(\Omega) \text { e } \varepsilon \in(0,1] \text {. }
$$


Pelas desigualdades 4.5 as normas de $L_{\varepsilon}^{2}(\Omega)$ são todas equivalentes a norma usual de $L^{2}(\Omega)$ com constante de equivalência independente de $\varepsilon$, de modo que, para $\varepsilon \in(0,1]$, as normas $\|\cdot\|_{\varepsilon}$ são também equivalentes a norma usual de $H^{1}(\Omega)$. Assim $H_{\mathcal{\varepsilon}}^{1}(\Omega)$ esta denso e compactamente imerso em $L_{\mathcal{\varepsilon}}^{2}(\Omega)$. Portanto se $A_{\varepsilon}: D\left(A_{\varepsilon}\right) \subset L_{\varepsilon}^{2}(\Omega) \rightarrow L_{\mathcal{\varepsilon}}^{2}(\Omega)\left(\right.$ resp. $\left.A_{0}: D\left(A_{0}\right) \subset L_{s}^{2}(\Omega) \rightarrow L_{S}^{2}(\Omega)\right)$ é o operador definido via a forma bilinear $a_{\varepsilon}$ (resp. $a_{0}$ ), então $A_{\varepsilon}$ (resp. $A_{0}$ ) é um operador auto-adjunto, positivo, setorial com resolvente compacto.

Teorema 4.7. A família $\left\{A_{\varepsilon}^{-1} \in \mathscr{L}\left(L_{\mathcal{\varepsilon}}^{2}(\Omega)\right)\right\} \stackrel{C C}{\longrightarrow} A_{0}^{-1} \in \mathscr{L}\left(L_{s}^{2}(\Omega)\right)$.

Demonstração: Seja $\left\{f_{\varepsilon}\right\}_{\varepsilon \in(0,1]}, f_{\varepsilon} \in L_{\varepsilon}^{2}(\Omega)$, uma família uniformemente limitada. Colocando $u_{\varepsilon}=A_{\varepsilon}^{-1} f_{\varepsilon}$ vemos que

$$
\int_{\Omega} J_{\mathcal{\varepsilon}}(x)\left(\left\langle S_{\mathcal{\varepsilon}}(x)^{T} \nabla u_{\mathcal{\varepsilon}}(x), S_{\mathcal{\varepsilon}}(x)^{T} \nabla u_{\varepsilon}(x)\right\rangle+\frac{1}{\varepsilon^{2}}\left\langle P(x) \nabla u_{\mathcal{\varepsilon}}(x), P(x) \nabla u_{\varepsilon}(x)\right\rangle+u_{\mathcal{\varepsilon}}(x)^{2}\right) d x=\left(u_{\varepsilon}, f_{\varepsilon}\right)_{\varepsilon} .
$$

Assim pela Proposição 4.4 e (4.8) existem constantes positivas $\eta$ e $\tilde{M}$ tais que

$$
\eta\left\|u_{\varepsilon}\right\|_{H^{1}(\Omega)}^{2} \leqslant\left\|u_{\mathcal{\varepsilon}}\right\|_{\varepsilon}^{2} \leqslant \tilde{M}\left\|f_{\mathcal{\varepsilon}}\right\|_{L_{\varepsilon}^{2}(\Omega)}\left\|u_{\varepsilon}\right\|_{H^{1}(\Omega)} \Rightarrow\left\|u_{\mathcal{\varepsilon}}\right\|_{H^{1}(\Omega)} \leqslant \frac{\tilde{M}}{\eta} .
$$

Logo existe $u_{0} \in H^{1}(\Omega)$ tal que $u_{\varepsilon} \rightarrow u_{0}$, fracamente em $H^{1}(\Omega)$ e fortemente em $L^{2}(\Omega)$. Afirmamos que $u_{0} \in H_{s}^{1}(\Omega)$. De fato, como

$$
\frac{1}{\varepsilon^{2}} \int_{\Omega} J_{\varepsilon}(x)\left\langle P(x) \nabla u_{\varepsilon}(x), P(x) \nabla u_{\varepsilon}(x)\right\rangle d x \leqslant \tilde{M}<\infty
$$

e $0<\tilde{m} \leqslant J_{\mathcal{\varepsilon}}(x)$ em $[0,1] \times \bar{\Omega}$, temos

$$
\int_{\Omega} J_{\varepsilon}(x)\left\langle P(x) \nabla u_{\varepsilon}(x), P(x) \nabla u_{\varepsilon}(x)\right\rangle d x \rightarrow 0,
$$

e $\operatorname{assim} P \nabla u_{\varepsilon} \rightarrow 0$ em $L^{2}\left(\Omega, \mathbb{R}^{n}\right)$. Uma vez que $\nabla u_{\varepsilon} \rightarrow \nabla u_{0}$ em $L^{2}\left(\Omega, \mathbb{R}^{n}\right)$ e a aplicação $\bar{\Omega} \ni x \mapsto$ $P(x) \in \mathscr{L}\left(\mathbb{R}^{n}, \mathbb{R}^{n}\right)$ é contínua, segue que $P \nabla u_{\varepsilon} \rightarrow P \nabla u_{0}$ em $L^{2}\left(\Omega, \mathbb{R}^{n}\right)$. Portanto $P(x) \nabla u_{0}(x)=0$ q.s. em $\Omega$.

O restante da prova segue como no Teorema 3.9 nos valendo da Proposição 4.4.

Lema 4.8. Sejam $\varepsilon_{n} \stackrel{n \rightarrow \infty}{\longrightarrow} 0$ e $\left\{f_{\varepsilon_{n}}\right\}, f_{\varepsilon_{n}} \in L_{\varepsilon_{n}}^{2}(\Omega)$, e $f_{0} \in L_{s}^{2}(\Omega)$ com $f_{\varepsilon_{n}} \stackrel{E}{\longrightarrow} f_{0}$. Ent $\tilde{a} o$ se $u_{\varepsilon_{n}}=A_{\varepsilon_{n}}^{-1} f_{\varepsilon_{n}}$ e $u_{0}=A_{0}^{-1} f_{0}$

$$
\lim _{n \rightarrow \infty}\left\|u_{\varepsilon_{n}}-u_{0}\right\|_{H^{1}(\Omega)}=0 .
$$

Demonstração: Observando a continuidade dos operadores $J_{\varepsilon}$ e $S_{\varepsilon}^{T}$ e a equivalência das normas $\|\cdot\|_{H^{1}(\Omega)}$ e $\|\cdot\|_{0}$, a demonstração segue como no Lema 3.12. 
Lema 4.9. Nas hipóteses do Lema anterior, sejam $\lambda_{\varepsilon}$ e $\lambda_{0}$ tais que

$$
\begin{aligned}
& \lambda_{\varepsilon}=\frac{1}{2}\left\|u_{\varepsilon}\right\|_{\varepsilon}^{2}-\left(f_{\varepsilon}, u_{\varepsilon}\right)_{\varepsilon}=\min _{\phi \in H_{\varepsilon}^{1}(\Omega)}\left(\frac{1}{2}\|\phi\|_{\varepsilon}^{2}-\int_{\Omega} J_{\varepsilon} f_{\varepsilon} \phi\right) \\
& \lambda_{0}=\frac{1}{2}\left\|u_{0}\right\|_{0}^{2}-\left(f_{0}, u_{0}\right)_{0}=\min _{\phi \in H_{s}^{1}(\Omega)}\left(\frac{1}{2}\|\phi\|_{0}^{2}-\int_{\Omega} J_{0} f_{0} \phi\right) .
\end{aligned}
$$

Então

$$
\lim _{\varepsilon \rightarrow 0} \lambda_{\varepsilon}=\lambda_{0}
$$

Demonstração: Pela Proposição 4.4, dado $\delta>0$, existe $\bar{\varepsilon}$ tal que se $0<\varepsilon<\bar{\varepsilon}$

$$
\begin{aligned}
\lambda_{\varepsilon} & \leqslant \frac{1}{2} \int_{\Omega} J_{\mathcal{\varepsilon}}\left(\left\langle S_{\varepsilon}^{T} \nabla u_{0}, S_{\varepsilon}^{T} \nabla u_{0}\right\rangle+u_{0}^{2}\right) d x-\left(f_{\varepsilon}, u_{0}\right)_{\varepsilon} \\
& \leqslant \frac{1}{2} \int_{\Omega} J_{0}\left(\left\langle S_{0}^{T} \nabla u_{0}, S_{0}^{T} \nabla u_{0}\right\rangle+u_{0}^{2}\right) d x+\delta-\int_{\Omega} J_{0} f_{0} u_{0} d x-\int_{\Omega}\left(J_{\varepsilon} f_{\varepsilon}-J_{0} f_{0}\right) u_{0} d x \\
& =\lambda_{0}+\delta-\int_{\Omega}\left(J_{\varepsilon} f_{\varepsilon}-J_{0} f_{0}\right) u_{0} d x
\end{aligned}
$$

Logo

$$
\limsup _{\varepsilon \rightarrow 0} \lambda_{\varepsilon} \leqslant \lambda_{0}
$$

Por outro lado,

$$
\begin{aligned}
\lambda_{0}= & \frac{1}{2} \int_{\Omega} J_{0}\left(\left|S_{0}^{T} \nabla u_{0}\right|^{2}+u_{0}^{2}\right)-\int_{\Omega} J_{0} f_{0} u_{0} \\
= & \frac{1}{2} \int_{\Omega} J_{0}\left(\left|S_{0}^{T} \nabla_{x} u_{0}-S_{\varepsilon}^{T} \nabla u_{\varepsilon}+S_{\varepsilon}^{T} \nabla u_{\varepsilon}\right|^{2}+\left|u_{0}-u_{\varepsilon}+u_{\varepsilon}\right|^{2}\right)-\int_{\Omega} J_{0} f_{0}\left(u_{0}-u_{\varepsilon}\right) \\
& -\int_{\Omega} J_{0}\left(f_{0}-f_{\varepsilon}\right) u_{\varepsilon}+\int_{\Omega} J_{\varepsilon} f_{\varepsilon} u_{\varepsilon}-\int_{\Omega}\left(J_{\varepsilon}-J_{0}\right) f_{\varepsilon} u_{\varepsilon} \\
= & \frac{1}{2} \int_{\Omega} J_{0}\left|S_{0}^{T} \nabla u_{0}-S_{\varepsilon}^{T} \nabla u_{\varepsilon}\right|^{2}+\int_{\Omega} J_{0}\left\langle S_{0}^{T} \nabla u_{0}-S_{\varepsilon}^{T} \nabla u_{\varepsilon}, S_{\varepsilon}^{T} \nabla u_{\varepsilon}\right\rangle+\frac{1}{2} \int_{\Omega} J_{0}\left(\left|S_{\varepsilon}^{T} \nabla u_{\varepsilon}\right|^{2}+\left|u_{\varepsilon}\right|^{2}\right) \\
& +\int_{\Omega} J_{0}\left(u_{0}-u_{\varepsilon}\right) u_{\varepsilon}+\frac{1}{2} \int_{\Omega} J_{0}\left|u_{0}-u_{\varepsilon}\right|^{2}-\int_{\Omega} J_{0} f_{0}\left(u_{0}-u_{\varepsilon}\right)-\int_{\Omega} J_{0}\left(f_{0}-f_{\varepsilon}\right) u_{\varepsilon}+\int_{\Omega} J_{\varepsilon} f_{\varepsilon} u_{\varepsilon}-\int_{\Omega}\left(J_{\varepsilon}-J_{0}\right) f_{\varepsilon} u_{\varepsilon} \\
\leqslant & \frac{\tilde{\delta}}{2}\left\|u_{0}-u_{\varepsilon}\right\|_{H^{1}(\Omega)}^{2}+\lambda_{\varepsilon}+\int_{\Omega}\left(J_{0}-J_{\varepsilon}\right)\left(\left|S_{\varepsilon}^{T} \nabla u_{\varepsilon}\right|^{2}+\left|u_{\varepsilon}\right|^{2}\right)+\int_{\Omega} J_{0}\left\langle S_{0}^{T} \nabla u_{0}-S_{\varepsilon}^{T} \nabla u_{\varepsilon}, S_{\varepsilon}^{T} \nabla u_{\varepsilon}\right\rangle \\
& +\int_{\Omega} J_{0}\left(u_{0}-u_{\varepsilon}\right) u_{\varepsilon}-\int_{\Omega} J_{0} f_{0}\left(u_{0}-u_{\varepsilon}\right)-\int_{\Omega} J_{0}\left(f_{0}-f_{\varepsilon}\right) u_{\varepsilon}
\end{aligned}
$$

E o resultado segue de (6.6) e do Lema anterior.

Lema 4.10. Sejam $\varepsilon_{n} \stackrel{n \rightarrow \infty}{\longrightarrow} 0$ e $\left\{f_{\varepsilon_{n}}\right\}, f_{\varepsilon_{n}} \in L_{\mathcal{E}_{n}}^{2}(\Omega)$, e $f_{0} \in L_{s}^{2}(\Omega)$ com $f_{\varepsilon_{n}} \stackrel{E}{\longrightarrow} f_{0}$. Então se $u_{\varepsilon_{n}}=$ $A_{\varepsilon_{n}}^{-1} f_{\varepsilon_{n}}$ e $u_{0}=A_{0}^{-1} f_{0}$

$$
\lim _{n \rightarrow \infty}\left\|u_{\varepsilon_{n}}-u_{0}\right\|_{\varepsilon_{n}}=0
$$


Demonstração: Como na prova do Lema anterior

$$
\begin{aligned}
\lambda_{\varepsilon} & =\frac{1}{2}\left\|u_{\varepsilon}\right\|_{\varepsilon}^{2}-\int_{\Omega} J_{\varepsilon} f_{\varepsilon} u_{\varepsilon} \\
& =\frac{1}{2}\left\|u_{\varepsilon}-u_{0}+u_{0}\right\|_{\varepsilon}^{2}-\int_{\Omega} J_{\varepsilon} f_{\varepsilon} u_{\varepsilon}-\int_{\Omega} J_{\varepsilon} f_{\varepsilon}\left(u_{\varepsilon}-u_{0}\right)-\int_{\Omega} J_{\varepsilon}\left(f_{\varepsilon}-f_{0}\right) u_{0}-\int_{\Omega} J_{\varepsilon} f_{0} u_{0} \\
& =\frac{1}{2}\left\|u_{\varepsilon}-u_{0}\right\|_{\varepsilon}^{2}+\frac{1}{2}\left\|u_{0}\right\|_{\varepsilon}^{2}-\int_{\Omega} J_{0} f_{0} u_{0}+\int_{\Omega}\left(J_{\varepsilon}-J_{0}\right) f_{0} u_{0} \\
& +\int_{\Omega}\left\langle S_{\varepsilon}\left(\nabla u_{\varepsilon}-\nabla u_{0}\right), \nabla u_{0}\right\rangle+\int_{\Omega} J_{\mathcal{\varepsilon}}\left(u_{\varepsilon}-u_{0}\right) u_{0}-\int_{\Omega} J_{\varepsilon} f_{\varepsilon}\left(u_{\varepsilon}-u_{0}\right)-\int_{\Omega} J_{\varepsilon}\left(f_{\varepsilon}-f_{0}\right) u_{0} .
\end{aligned}
$$

Observando que $\left\|u_{0}\right\|_{\varepsilon} \rightarrow\left\|u_{0}\right\|_{0}$ o resultado segue do Lema anterior.

Corolário 4.11. Seja $\varepsilon_{n} \rightarrow 0$. Se $f_{\mathcal{E}_{n}} \in L_{\varepsilon_{n}}^{2}(\Omega)$ E-converge para $f_{0} \in L_{s}^{2}(\Omega)$, então

$$
\left|a_{\varepsilon_{n}}\left(u_{\varepsilon_{n}}, u_{\varepsilon_{n}}\right)-a_{0}\left(u_{0}, u_{0}\right)\right| \rightarrow 0,
$$

onde $u_{\varepsilon_{n}}:=A_{\varepsilon_{n}}^{-1} f_{\mathcal{E}_{n}}$ e $u_{0}:=A_{0}^{-1} f_{0}$.

Demonstração: Iniciemos calculando

$$
\begin{aligned}
\left|a_{\varepsilon_{n}}\left(u_{\varepsilon_{n}}, u_{\mathcal{E}_{n}}\right)-a_{0}\left(u_{0}, u_{0}\right)\right| \leqslant & \left|a_{\varepsilon_{n}}\left(u_{\varepsilon_{n}}, u_{\varepsilon_{n}}\right)+\left(u_{\varepsilon_{n}}, u_{\varepsilon_{n}}\right) \varepsilon_{\varepsilon_{n}}-a_{\varepsilon_{n}}\left(u_{0}, u_{0}\right)-\left(u_{0}, u_{0}\right)_{\varepsilon_{n}}\right| \\
& +\left|a_{\varepsilon_{n}}\left(u_{0}, u_{0}\right)-a_{0}\left(u_{0}, u_{0}\right)\right|+\left|\left(u_{\varepsilon_{n}}, u_{\varepsilon_{n}}\right)_{\varepsilon_{n}}-\left(u_{0}, u_{0}\right)_{\varepsilon_{n}}\right| \\
= & ||\left|u_{\varepsilon_{n}}\right|_{\varepsilon_{n}}^{2}-|| u_{0} \|_{\mathcal{E}_{n}}^{2}|+| a_{\mathcal{\varepsilon}_{n}}\left(u_{0}, u_{0}\right)-a_{0}\left(u_{0}, u_{0}\right)|+|\left(u_{\varepsilon_{n}}, u_{\varepsilon_{n}}\right)_{\varepsilon_{n}}-\left(u_{0}, u_{0}\right)_{\varepsilon_{n}} \mid
\end{aligned}
$$

Agora pelo Lema 4.10

$$
\left|\left\|u_{\varepsilon_{n}}\right\|_{\varepsilon_{n}}-\left\|u_{0}\right\|_{\varepsilon_{n}}\right| \leqslant\left\|u_{\varepsilon_{n}}-u_{0}\right\|_{\mathcal{E}_{n}} \rightarrow 0
$$

e

$$
\left|\left\|\left.u_{\varepsilon_{n}}\right|_{L_{\varepsilon_{n}}^{2}(\Omega)}-\right\| u_{0}\left\|_{L_{\varepsilon_{n}}^{2}(\Omega)} \mid \leqslant\right\| u_{\varepsilon_{n}}-u_{0} \|_{L_{\varepsilon_{n}}^{2}(\Omega)} \rightarrow 0 .\right.
$$

Além disso, temos pela Proposição 4.6,

$$
\left\|u_{0}\right\|_{\varepsilon_{n}} \rightarrow\left\|u_{0}\right\|_{0} \quad \text { e } \quad\left\|u_{0}\right\|_{L_{\varepsilon_{n}}^{2}(\Omega)} \rightarrow\left\|u_{0}\right\|_{L_{s}^{2}(\Omega)}
$$

e o resultado segue. 



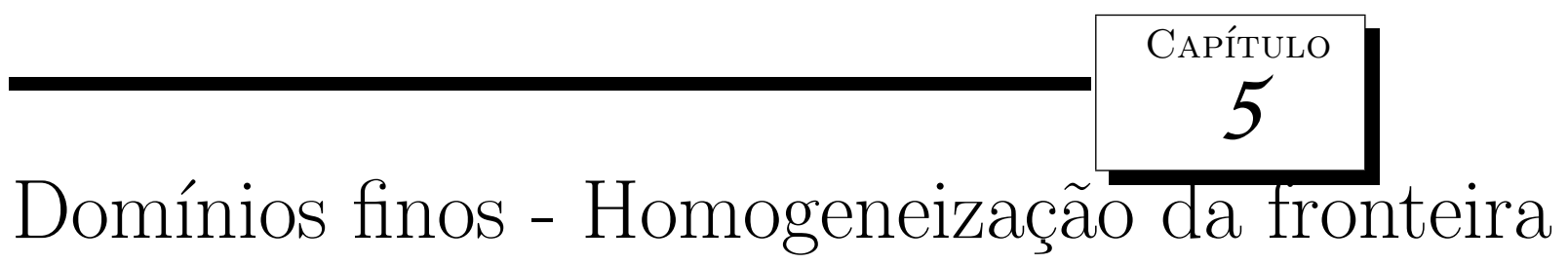

Neste exemplo mostraremos um análogo dos resultados anteriores para o caso em que o domínio espacial se colapsa a um domínio de dimensão inferior tal que há uma região de sua fronteira que apresenta um comportamento altamente oscilante.

Consideremos uma família de funções $k_{\varepsilon} \in C^{1}\left(\mathbb{R}^{m+n}\right)$ uniformemente limitada, i.e., existe uma constante $K>0$ tal que $\left|\sup _{\varepsilon} \sup _{x \in \mathbb{R}^{m+n}} k_{\varepsilon}(x)\right| \leqslant K$. Supomos ainda que $\sup _{x \in \mathbb{R}^{m+n}} k_{\varepsilon}(x) \stackrel{\varepsilon \rightarrow 0}{\longrightarrow} 0$. Como nos demais capítulos, nosso interesse é estudar o comportamento assintótico de uma equação de reação-difusão em um domínio fino. Entretanto neste caso há uma região da fronteira que apresenta um comportamento irregular determinado pela família $k_{\varepsilon}$. Seja a família de domínios $\Omega_{\varepsilon}:=\left\{\left(x,\left(k_{\varepsilon}(x)+1\right) y\right) \in R^{m+n}:(x, y) \in \Omega\right\}$.

Consideremos a equação

$$
\begin{array}{ll}
u_{t}=\Delta u-u+f(u), & (0, \infty) \times R_{\varepsilon} \\
\frac{\partial u}{\partial \eta_{\varepsilon}}=0, & (0, \infty) \times \partial R_{\varepsilon}
\end{array}
$$

onde $R_{\varepsilon}=\left\{(x, \varepsilon y),(x, y) \in \Omega_{\varepsilon}\right\}$, é a $y$-compressão pelo fator $\varepsilon$ do domínio $\Omega_{\varepsilon}$ e $\eta_{\varepsilon}$ é o vetor normal unitário exterior a $\partial R_{\mathcal{\varepsilon}}$.

Observemos que $R_{\varepsilon}=T_{\varepsilon}\left(\Omega_{\varepsilon}\right)$, onde $T_{\varepsilon}: \mathbb{R}^{m+n} \rightarrow \mathbb{R}^{m+n}$ é dada por $T_{\varepsilon}(x, y)=(x, \varepsilon y)$. Através de $T_{\mathcal{E}}$, de maneira análoga ao Capítulo 3 obtemos uma conjugação entre o problema $\left(\tilde{P}_{\varepsilon}\right)$ e o problema

$$
\begin{array}{ll}
u_{t}=\Delta_{x} u+\frac{1}{\varepsilon^{2}} \Delta_{y} u-u+f(u), & (0, \infty) \times \Omega_{\varepsilon} \\
\frac{\partial u}{\partial \eta_{x}}+\frac{1}{\varepsilon^{2}} \frac{\partial u}{\partial \eta_{y}}=0, & (0, \infty) \times \partial \Omega_{\varepsilon}
\end{array}
$$

definido em $\Omega_{\varepsilon}$. Analogamente mostraremos que a dinâmica da equação $\left(P_{\varepsilon}\right)$ pode ser aproximada pela dinâmica de uma equação abstrata $\left(P_{0}\right)$ definida em um subespaço fechado de $H^{1}(\Omega)$. Para isso introduzimos novamente os espaços $H_{s}^{1}(\Omega):=\left\{u \in H^{1}(\Omega): \nabla_{y} u=0\right\}$ e $L_{s}^{2}(\Omega):=$ fecho de $H_{s}^{1}(\Omega)$ em $L^{2}(\Omega)$. Para um subconjunto aberto $O$ de $\mathbb{R}^{m+n}$, seja $H_{\varepsilon}^{1}(O)$ o espaço $H^{1}(O)$ munido da norma

$$
\|u\|_{H_{\varepsilon}^{1}(O)}:=\left(\int_{O}\left(\left|\nabla_{x} u\right|^{2}+\frac{1}{\varepsilon^{2}}\left|\nabla_{y} u\right|^{2}+|u|^{2}\right) d x d y\right)^{\frac{1}{2}} .
$$


Os semigrupos associados as equações $\left(P_{\varepsilon}\right)$ e $\left(P_{0}\right)$ serão comparados no espaço $\mathscr{H}_{\varepsilon}^{1}=H_{\varepsilon}^{1}\left(\Omega_{\varepsilon} \cap \Omega\right) \oplus H_{\varepsilon}^{1}\left(\Omega_{\varepsilon} \backslash \bar{\Omega}\right) \oplus H_{\varepsilon}^{1}\left(\Omega \backslash \bar{\Omega}_{\varepsilon}\right)$, i.e.

$$
\mathscr{H}_{\varepsilon}^{1}=\left\{u \in L^{2}\left(\Omega_{\varepsilon} \cup \Omega\right): u_{\mid \Omega_{\varepsilon} \cap \Omega} \in H^{1}\left(\Omega_{\varepsilon} \cap \Omega\right), u_{\mid \Omega_{\varepsilon} \backslash \bar{\Omega}} \in H^{1}\left(\Omega_{\varepsilon} \backslash \bar{\Omega}\right), u_{|\Omega| \Omega_{\varepsilon}} \in H^{1}\left(\Omega \backslash \bar{\Omega}_{\varepsilon}\right)\right\},
$$

munido da norma $\|u\|_{\mathscr{H}_{\varepsilon}^{1}}^{2}=\|u\|_{H_{\varepsilon}^{1}\left(\Omega_{\varepsilon} \cap \Omega\right)}^{2}+\|u\|_{H_{\varepsilon}^{1}\left(\Omega_{\varepsilon} \backslash \bar{\Omega}\right)}^{2}+\|u\|_{H_{\varepsilon}^{1}\left(\Omega \backslash \bar{\Omega}_{\varepsilon}\right)}^{2}$.

Consideremos os operadores $E_{\varepsilon} \in \mathscr{L}\left(H_{s}^{1}(\Omega), H_{\varepsilon}^{1}\left(\Omega_{\varepsilon}\right)\right)$, (resp. $\left.E_{\varepsilon} \in \mathscr{L}\left(L_{s}^{2}(\Omega), L_{\varepsilon}^{2}\left(\Omega_{\varepsilon}\right)\right)\right)$, dados por

$$
E_{\varepsilon}=R_{\varepsilon} \circ E
$$

onde $E: H^{1}(\Omega) \rightarrow H^{1}\left(\mathbb{R}^{m+n}\right),\left(\right.$ resp. $\left.E: L^{2}(\Omega) \rightarrow L^{2}\left(\mathbb{R}^{m+n}\right)\right)$ estende uma função definida em $\Omega$ a uma função definida em todo $\mathbb{R}^{m+n}$, de modo que para funções $u \in H_{s}^{1}(\Omega)$ ainda tenhamos $E u \in H_{s}^{1}\left(\mathbb{R}^{m+n}\right)$ e $R_{\varepsilon}: H^{1}\left(\mathbb{R}^{m+n}\right) \rightarrow H^{1}\left(\Omega_{\varepsilon}\right)\left(\right.$ resp. $\left.R_{\varepsilon}: L^{2}\left(\mathbb{R}^{m+n}\right) \rightarrow L^{2}\left(\Omega_{\varepsilon}\right)\right)$ é a restrição a $\Omega_{\varepsilon}$.

Observamos que ao estendermos as funções de $H^{1}\left(\Omega_{\varepsilon}\right)$ (resp. $L^{2}\left(\Omega_{\varepsilon}\right)$ ) por zero fora de $\Omega_{\varepsilon}$, temos $H_{\varepsilon}^{1}\left(\Omega_{\varepsilon}\right) \hookrightarrow \mathscr{H}_{\varepsilon}^{1}\left(\right.$ resp. $\quad L^{2}\left(\Omega_{\varepsilon}\right) \hookrightarrow L^{2}\left(\Omega_{\varepsilon} \cup \Omega\right)$ ) com constante de imersão 1. Assim, se $u_{\varepsilon} \in H_{\varepsilon}^{1}\left(\Omega_{\varepsilon}\right)$ e $u \in H_{s}^{1}(\Omega)$, tem sentido $\left\|u_{\varepsilon}-E_{\varepsilon} u\right\|_{\mathscr{H}_{\varepsilon}^{1}}$ e podemos escrever $E_{\varepsilon} \in \mathscr{L}\left(H_{s}^{1}(\Omega), \mathscr{H}_{\varepsilon}^{1}\right)$.

Devido as nossas hipóteses sobre as funções $k_{\varepsilon}$, é claro que $\left|\Omega_{\varepsilon} \backslash \Omega\right|+\left|\Omega \backslash \Omega_{\varepsilon}\right| \rightarrow 0$, quando $\varepsilon \rightarrow 0$. Com isso e da construção dos operadores $E_{\varepsilon}$ fica fácil vermos que se $u \in H_{s}^{1}(\Omega)$ então $\left\|E_{\varepsilon} u\right\|_{\mathscr{H}_{\varepsilon}^{1}} \rightarrow\|u\|_{H_{s}^{1}(\Omega)}$, quando $\varepsilon \rightarrow 0$. De fato,

$$
\left\|E_{\varepsilon} u\right\|_{\mathscr{H}_{\varepsilon}^{1}}^{2}=\int_{\Omega}\left(|\nabla u|^{2}+|u|^{2}\right) d x d y+\int_{\Omega_{\varepsilon} \backslash \Omega}\left(\left|\nabla_{x} E u\right|^{2}+|E u|^{2}\right) d x d y \rightarrow\|u\|_{H_{s}^{1}(\Omega)}^{2}
$$

Além disso, se $K \subset \subset \Omega$, então existe $\varepsilon(K)$ tal que $K \subset \subset \Omega_{\varepsilon}, 0<\varepsilon<\varepsilon(K)$. Esta hipótese foi fortemente empregada nos trabalhos [3], [5] e [6].

Introduzimos as formas bilineares

$$
\begin{gathered}
a_{\varepsilon}: H_{\varepsilon}^{1}\left(\Omega_{\varepsilon}\right) \times H_{\varepsilon}^{1}\left(\Omega_{\varepsilon}\right) \rightarrow \mathbb{R} \\
a_{\varepsilon}(u, v):=\int_{\Omega_{\varepsilon}}\left(\nabla_{x} u \cdot \nabla_{x} v+\frac{1}{\varepsilon^{2}} \nabla_{y} u \cdot \nabla_{y} v+u v\right) d x d y
\end{gathered}
$$

que induz um operador linear fechado, positivo, auto-adjunto e com resolvente compacto

$$
A_{\varepsilon}: D\left(A_{\varepsilon}\right) \subset L^{2}\left(\Omega_{\varepsilon}\right) \rightarrow L^{2}\left(\Omega_{\varepsilon}\right),
$$

$\operatorname{com} D\left(A_{\varepsilon}\right)=\left\{u \in H^{2}\left(\Omega_{\varepsilon}\right): \frac{\partial u}{\partial \eta_{x}}+\frac{1}{\varepsilon^{2}} \frac{\partial u}{\partial \eta_{y}}=0\right\}, \mathrm{e}$

$$
\begin{gathered}
a_{0}: H_{s}^{1}\left(\Omega_{0}\right) \times H_{s}^{1}\left(\Omega_{0}\right) \rightarrow \mathbb{R} \\
a_{0}(u, v):=\int_{\Omega_{0}}(\nabla u \cdot \nabla v+u v) d x d y,
\end{gathered}
$$

que induz um operador linear fechado, positivo, auto-adjunto e com resolvente compacto

$$
A_{0}: D\left(A_{0}\right) \subset L_{s}^{2}(\Omega) \rightarrow L_{s}^{2}(\Omega)
$$


Proposição 5.1. A família $A_{\varepsilon}^{-1} \in \mathscr{L}\left(L^{2}\left(\Omega_{\varepsilon}\right)\right)$ converge compactamente para o operador $A^{-1} \in$ $\mathscr{L}\left(L_{s}^{2}(\Omega)\right)$.

Demonstração: Seja $\left\{f_{\varepsilon}\right\}_{\varepsilon \in(0,1]}$ uma família uniformemente limitada, isto é, $\left\|f_{\mathcal{\varepsilon}}\right\|_{L^{2}\left(\Omega_{\varepsilon}\right)}=1$, colocando $u_{\varepsilon}=A_{\varepsilon}^{-1} f_{\varepsilon}$ vemos que

$$
a_{\varepsilon}\left(u_{\varepsilon}, v\right)=\left(f_{\varepsilon}, v\right)_{L^{2}\left(\Omega_{\varepsilon}\right)}, \quad \forall v \in H^{1}\left(\Omega_{\varepsilon}\right)
$$

em particular, $a_{\varepsilon}\left(u_{\varepsilon}, u_{\varepsilon}\right)=\left(f_{\varepsilon}, u_{\varepsilon}\right)_{L^{2}\left(\Omega_{\varepsilon}\right)}$. Mas

$$
\left\|u_{\mathcal{\varepsilon}}\right\|_{H^{1}\left(\Omega_{\varepsilon}\right)}^{2} \leqslant a_{\varepsilon}\left(u_{\varepsilon}, u_{\varepsilon}\right) \leqslant\left\|f_{\varepsilon}\right\|_{L^{2}\left(\Omega_{\varepsilon}\right)}\left\|u_{\varepsilon}\right\|_{H^{1}\left(\Omega_{\varepsilon}\right)},
$$

e portanto $\left\|u_{\varepsilon}\right\|_{H^{1}\left(\Omega_{\varepsilon}\right)} \leqslant 1$.

Logo, a menos de subseqüências, existe $u_{0} \in H^{1}(\Omega)$ tal que $u_{\varepsilon} \rightarrow u_{0}$, fracamente em $H^{1}(K)$ e fortemente em $L^{2}(K)$, qualquer que seja $K \subset \subset \Omega$.

Além disso, como $a_{\varepsilon}\left(u_{\varepsilon}, u_{\varepsilon}\right) \leqslant 1$ é claro que $\nabla_{y} u_{\varepsilon} \rightarrow 0$. Agora se $\phi \in C_{0}^{\infty}(\Omega)$ então

$$
\int_{\Omega} u_{0} \phi_{y} \leqslant\left\|u_{0}-u_{\varepsilon}\right\|_{L^{2}\left(K_{\phi}\right)}\left\|\phi_{y}\right\|_{L^{2}(\Omega)}+\left\|\nabla_{y} u_{\varepsilon}\right\|_{L^{2}(\Omega)}\|\phi\|_{L^{2}(\Omega)} \stackrel{\varepsilon \rightarrow 0}{\longrightarrow} 0
$$

onde $K_{\phi}=\operatorname{supp} \phi$. Ou seja, $u_{0} \in H_{s}^{1}(\Omega)$. Agora como $\left|\Omega_{\varepsilon} \backslash \Omega\right| \rightarrow 0$, não é difícil ver que $A_{\varepsilon}^{-1} f_{\varepsilon} \stackrel{E}{\longrightarrow}$ $u_{0}$.

Consideremos agora uma seqüencia $f_{\varepsilon} \stackrel{E}{\longrightarrow} f_{0}$. Então $\left\{f_{\varepsilon}\right\}$ é uniformemente limitada, e novamente se $A_{\varepsilon}^{-1} f_{\varepsilon}=u_{\varepsilon}$ existe $u_{0} \in H_{s}^{1}(\Omega)$ tal que $u_{\varepsilon} \stackrel{E}{\longrightarrow} u_{0}$.

Mostremos que $u_{0}=A_{0}^{-1} f_{0}$, isto é, mostremos que $a_{0}\left(u_{0}, v\right)=\left(f_{0}, v\right)_{L^{2}(\Omega)}, \forall v \in H_{s}^{1}(\Omega)$.

Já que $f_{\varepsilon} \stackrel{E}{\longrightarrow} f_{0}$ e $u_{\varepsilon} \stackrel{E}{\longrightarrow} u_{0}$ temos $\left(f_{\varepsilon}, E_{\varepsilon} v\right)_{L^{2}\left(\Omega_{\varepsilon}\right)} \rightarrow\left(f_{0}, v\right)_{L^{2}(\Omega)}$ e $\left(u_{\varepsilon}, E_{\varepsilon} v\right)_{L^{2}\left(\Omega_{\varepsilon}\right)} \rightarrow$ $\left(u_{0}, v\right)_{L^{2}(\Omega)}, \forall v \in H_{s}^{1}(\Omega)$. Além disso, se $K \subset \subset \Omega$ então $\int_{K} \nabla_{x} u_{\varepsilon} \cdot \nabla_{x} E_{\varepsilon} v \rightarrow \int_{K} \nabla u_{0} \cdot \nabla v, \forall v \in H_{s}^{1}(\Omega)$. Como $K$ pode ser escolhido arbitrário de modo a $\left|\Omega_{\varepsilon} \backslash K\right| \rightarrow 0$, não é difícil concluir que $a_{\varepsilon}\left(u_{\varepsilon}, E_{\varepsilon} v\right) \rightarrow a_{0}\left(u_{0}, v\right), \forall v \in H_{s}^{1}(\Omega)$.

Observando que $a_{\varepsilon}\left(u_{\varepsilon}, E_{\varepsilon} v\right)=\left(f_{\varepsilon}, E_{\varepsilon} v\right)_{L^{2}\left(\Omega_{\varepsilon}\right)}, \forall v \in H_{s}^{1}(\Omega)$ temos o resultado.

Agora introduzimos um complemento à teoria abstrata do Capítulo 2 provando resultados de convergência fraca em espaços de Hilbert, sempre levando em conta o que os espaços envolvidos variam de acordo com o parâmetro $\varepsilon$.

Definição 5.2. Sejam $H_{\varepsilon}$ espaços de Hilbert com produto interno e norma $(\cdot, \cdot)_{\varepsilon}$ e $\|\cdot\|_{H_{\varepsilon}}$ respectivamente. Dizemos que uma família $\left\{u_{\varepsilon}\right\}_{\varepsilon \in(0,1]}$ de elementos $u_{\varepsilon} \in H_{\varepsilon}$, E-converge fracamente para um elemento $u \in H_{0}$, se para qualquer familia $\left\{w_{\varepsilon}\right\}_{\varepsilon \in(0,1]}$ E-convergente para $w$, implica $\left(w_{\varepsilon}, u_{\varepsilon}\right)_{\varepsilon} \rightarrow(w, u)_{0}$ quando $\varepsilon \rightarrow 0$, e escrevemos $u_{\varepsilon} \stackrel{E}{\rightarrow} u$. 
Proposição 5.3. Se $\left\|u_{\varepsilon}\right\|_{H_{\varepsilon}} \leqslant M, u \in H_{0}$ e para todo $w \in H_{0},\left(E_{\varepsilon} w, u_{\varepsilon}\right)_{\varepsilon} \rightarrow(w, u)_{0}$ quando $\varepsilon \rightarrow 0$, então $u_{\varepsilon} \stackrel{E}{\rightarrow} u$.

Demonstração: Seja $w_{\varepsilon} \stackrel{E}{\longrightarrow} w$. Como $E_{\varepsilon} w \stackrel{E}{\longrightarrow} w$, então

$$
\left(w_{\varepsilon}, u_{\varepsilon}\right)_{\varepsilon}=\left(w_{\varepsilon}-E_{\varepsilon} w, u_{\varepsilon}\right)_{\varepsilon}-\left(E_{\varepsilon} w, u_{\varepsilon}\right)_{\varepsilon} \rightarrow(w, u)_{\varepsilon} .
$$

Proposição 5.4. Se $u_{\varepsilon} \stackrel{E}{\rightarrow} u$ e $\lim \sup _{\varepsilon \rightarrow 0}\left\|u_{\varepsilon}\right\|_{H_{\varepsilon}} \leqslant\|u\|_{H_{0}}$ então $u_{\varepsilon} \stackrel{E}{\longrightarrow} u$.

Demonstração: Basta observar que para cada $u \in H_{0}, E_{\varepsilon} u \stackrel{E}{\longrightarrow} u$ e então

$$
0 \leqslant\left\|u_{\varepsilon}-E_{\varepsilon} u\right\|_{H_{\varepsilon}}^{2}=\left\|u_{\varepsilon}\right\|_{H_{\varepsilon}}^{2}-2\left(u_{\varepsilon}, E_{\varepsilon} u\right)_{\varepsilon}+\left\|E_{\varepsilon} u\right\|_{H_{\varepsilon}}^{2} \rightarrow 0
$$

Proposição 5.5. Seja $u_{\varepsilon} \in \mathscr{H}_{\varepsilon}^{1}$ com $\left\|u_{\varepsilon}\right\|_{\mathscr{H}_{\varepsilon}^{1}} \leqslant M$. Então existe uma subseqüência também denotada por $u_{\varepsilon}$ e $u \in H_{s}^{1}(\Omega)$ tal que $u_{\varepsilon} \stackrel{E}{\rightarrow} u$.

Demonstração: A menos de subseqüências existe uma função $u \in H_{s}^{1}(\Omega)$ com a propriedade que $u_{\varepsilon} \rightarrow u$ fracamente em $H^{1}(K)$ e fortemente em $L^{2}(K)$, para qualquer $K \subset \subset \Omega$. Para mostrarmos que $u_{\varepsilon} \stackrel{E}{\rightarrow} u$ é suficiente mostrarmos que $\left(u_{\varepsilon}, E_{\varepsilon} v\right)_{\mathscr{H}_{\varepsilon}^{1}} \rightarrow(u, v)_{H^{1}(\Omega)}$ para todo $v \in H_{s}^{1}(\Omega)$. Lembrando que nosso operador de extensão $E$ leva o subespaço $H_{s}^{1}(\Omega)$ de $H^{1}(\Omega)$ no subespaço $H_{s}^{1}\left(\mathbb{R}^{m+n}\right)$ de $H^{1}\left(\mathbb{R}^{m+n}\right)$ temos para $v \in H_{s}^{1}(\Omega)$

$$
\begin{aligned}
\left(u_{\varepsilon}, E_{\varepsilon} v\right)_{\mathscr{H}_{\varepsilon}^{1}}-(u, v)_{H^{1}(\Omega)} & =\left(u_{\varepsilon}, E_{\varepsilon} v\right)_{H_{\varepsilon}^{1}\left(\Omega_{\varepsilon} \cap \Omega\right)}+\left(u_{\varepsilon}, E_{\varepsilon} v\right)_{H_{\varepsilon}^{1}\left(\Omega_{\varepsilon} \backslash \Omega\right)}+\left(u_{\varepsilon}, E_{\varepsilon} v\right)_{H_{\varepsilon}^{1}\left(\Omega \backslash \Omega_{\varepsilon}\right)}-(u, v)_{H^{1}(\Omega)} \\
& =\left(u_{\varepsilon}-u, v\right)_{H^{1}\left(\Omega_{\varepsilon} \cap \Omega\right)}+\left(u_{\varepsilon}, E_{\varepsilon} v\right)_{H^{1}\left(\Omega_{\varepsilon} \backslash \Omega\right)}+\left(u_{\varepsilon}-u, v\right)_{H^{1}\left(\Omega \backslash \Omega_{\varepsilon}\right.} .
\end{aligned}
$$

Observemos que $\left(u_{\varepsilon}-u, v\right)_{H^{1}\left(\Omega_{\varepsilon} \cap \Omega\right)} \rightarrow 0$ e $\left(u_{\varepsilon}-u, v\right)_{H^{1}\left(\Omega \backslash \Omega_{\varepsilon}\right)} \rightarrow 0$ devido a convergência fraca em $H^{1}\left(\Omega_{\varepsilon} \cap \Omega\right)$ e $H^{1}\left(\Omega \backslash \Omega_{\varepsilon}\right)$ respectivamente, e a outra parcela evidentemente tende a zero.

Proposição 5.6. A família $\left\{A_{\varepsilon}^{-1} \in \mathscr{L}\left(\mathscr{H}_{\varepsilon}^{1}\right)\right\}_{\varepsilon \in(0,1]}$ converge compactamente para o operador $A_{0}^{-1} \in \mathscr{L}\left(H_{s}^{1}(\Omega)\right)$.

Demonstração: Seja agora uma família de elementos $f_{\varepsilon} \in \mathscr{H}_{\varepsilon}^{1}\left(\Omega_{\varepsilon}\right)$ uniformemente limitada. Pela Proposição anterior, existe $f_{0} \in H_{s}^{1}(\Omega)$ tal que $f_{\varepsilon} \stackrel{E}{\rightarrow} f_{0}$. Como na prova da Proposição 5.1 podemos mostrar que a família $u_{\varepsilon}=A_{\varepsilon}^{-1} f_{\varepsilon}$ é também uniformemente limitada. Agora, novamente pela Proposição anterior, existe $u_{0} \in H_{s}^{1}(\Omega)$ tal que $u_{\varepsilon} \stackrel{E}{\rightarrow} u_{0}$. Afirmamos que de fato $u_{\varepsilon} \stackrel{E}{\longrightarrow} u_{0}$. Basta observar que,

$$
\left\|u_{\varepsilon}\right\|_{H_{\varepsilon}^{1}\left(\Omega_{\varepsilon}\right)}^{2}=\left(f_{\varepsilon}, u_{\varepsilon}\right)_{L^{2}\left(\Omega_{\varepsilon}\right)} \rightarrow\left(f_{0}, u_{0}\right)_{L_{s}^{2}(\Omega)}=\left\|u_{0}\right\|_{H_{s}^{1}(\Omega)}^{2}
$$


e concluir pela Proposição 5.4.

Além disso $u_{0}=A^{-1} f_{0}$, pois

$$
\left(u_{\mathcal{\varepsilon}}, E_{\varepsilon} v\right)_{H_{\varepsilon}^{1}\left(\Omega_{\varepsilon}\right)}=\left(f_{\mathcal{\varepsilon}}, E_{\varepsilon} v\right)_{L^{2}\left(\Omega_{\varepsilon}\right)} \rightarrow\left(f_{0}, v\right)_{L_{s}^{2}(\Omega)}=\left(A_{0}^{-1} f_{0}, v\right)_{H_{s}^{1}(\Omega)}
$$

Se assumirmos agora que $f_{\varepsilon} \stackrel{E}{\longrightarrow} f_{0}$, então é claro que a família $f_{\varepsilon} \in \mathscr{H}_{\varepsilon}^{1}\left(\Omega_{\varepsilon}\right)$ é uniformemente limitada e a proposição segue pelos argumentos acima. 



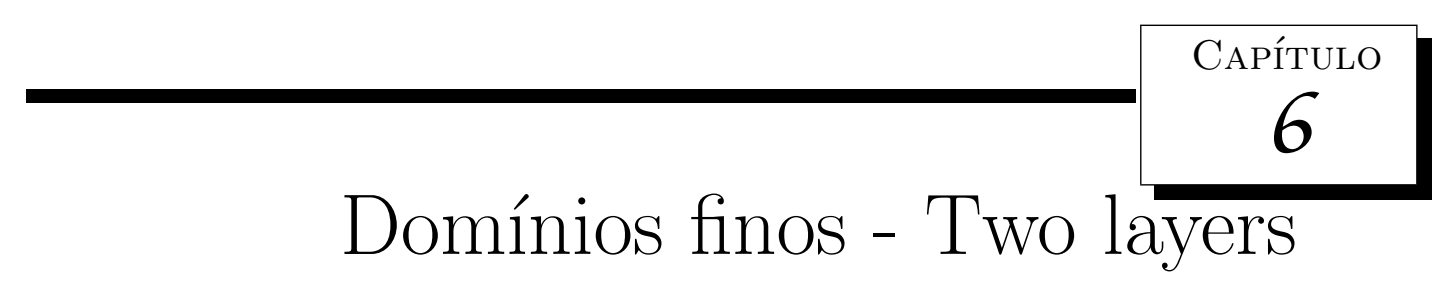

Como descrito na introdução, neste capítulo apresentamos um modelo que descreve o comportamento de uma equação de reação-difusão cujo domínio espacial consiste de duas componentes finas adjacentes $\Omega_{1, \varepsilon}$ e $\Omega_{2, \varepsilon}$ separadas por uma membrana permeável $\Gamma$. Supomos que a interação dos reagentes ocorre somente na interface $\Gamma$. Como conseqüência veremos que a intensidade da reação química em $\Gamma$ dependerá de quão fino forem os domínios preenchidos pelos reagentes. Este fenômeno será ditado pelo parâmetro $\varepsilon \in[0,1)$.

Sejam $\Omega_{1, \varepsilon}$ e $\Omega_{2, \varepsilon}$ domínios limitados de $\mathbb{R}^{m+1}$ com a seguinte estrutura

$$
\Omega_{1, \varepsilon}=\Gamma \times(0, \varepsilon), \quad \Omega_{2, \varepsilon}=\Gamma \times(-\varepsilon, 0),
$$

onde $\Gamma$ é um domínio limitado de $\mathbb{R}^{m}$ com fronteira $\partial \Gamma$ suave. Para um ponto $\xi \in \Omega_{\varepsilon}=\Omega_{1, \varepsilon} \cup \Omega_{2, \varepsilon}$, escrevemos $\xi=(x, y)$, onde $x \in \Gamma$ e $y \in(-\varepsilon, 0) \cup(0, \varepsilon)$.

Consideremos o seguinte sistema de equações parabólicas semilineares

$$
\begin{array}{ll}
\partial_{t} w_{i}=\Delta w_{i}-w_{i}+f_{i}\left(w_{i}\right), & (0, \infty) \times \Omega_{i, \varepsilon} \\
\frac{\partial w_{i}}{\partial \eta_{i}}=0, & (0, \infty) \times \partial \Omega_{i, \varepsilon} \backslash \Gamma \\
\frac{\partial w_{i}}{\partial \eta_{i}}-(-1)^{i} k(x)\left(w_{1}-w_{2}\right)=0, & (0, \infty) \times \Gamma
\end{array}
$$

onde $\eta_{i}$ é a normal exterior a $\partial \Omega_{i, \varepsilon}$.

Com relação as não linearidades $f_{i}$ assumiremos que sejam de classe $C^{2}$ com derivadas limitadas até segunda ordem e suporemos também que a aplicação $k \in C^{1}(\bar{\Gamma})$ é tal que $\inf _{x \in \Gamma} k(x)>0$.

Sob estas hipóteses o problema $\left(\tilde{P}_{\varepsilon}\right)$ gera um semigrupo $\tilde{T}_{\varepsilon}(t)(\cdot, \cdot):=\left(u_{1, \varepsilon}(t, \cdot), u_{2, \varepsilon}(t, \cdot)\right)$ atuando no espaço $H^{1}\left(\Omega_{1, \varepsilon}\right) \oplus H^{1}\left(\Omega_{2, \varepsilon}\right)$ que possui um atrator global $\tilde{\mathscr{A}}$. Mostraremos que a dinâmica da equação $\left(\tilde{P}_{\varepsilon}\right)$ pode ser aproximada pela dinâmica da seguinte equação definida em $\Gamma$

$$
\begin{aligned}
& \partial_{t} w=\Delta w-w+f(w), \quad \Gamma \\
& \frac{\partial w}{\partial \eta}=0, \quad \partial \Gamma,
\end{aligned}
$$

onde $f=\frac{1}{2}\left(f_{1}+f_{2}\right)$ e $\eta$ é a normal exterior unitária a $\partial \Gamma$. O que corresponde a sincronização dos componentes $u_{1, \varepsilon}$ e $u_{2, \varepsilon}$ da equação $\left(\tilde{P}_{\varepsilon}\right)$. 
Reescalando o domínio da equação $\left(\tilde{P}_{\varepsilon}\right)$ através da transformação $T_{\varepsilon}$ do capítulo 3 , transformamos o domínio $\Omega_{\varepsilon}$ no domínio $\Omega=\Omega_{1} \cup \Omega_{2}$, onde $\Omega_{1}=\Gamma \times(0,1)$ e $\Omega_{2}=\Gamma \times(-1,0)$. O problema anterior toma a forma

$$
\begin{array}{ll}
\partial_{t} w_{i}=\Delta_{x} w_{i}+\frac{1}{\varepsilon^{2}} \Delta_{y} w_{i}-w_{i}+f_{i}\left(w_{i}\right), & \Omega_{i} \\
\frac{\partial w_{i}}{\partial \eta_{i}}=0, & \partial \Omega_{i} \backslash \Gamma \\
\frac{\partial w_{i}}{\partial y}-\varepsilon k(x)\left(w_{1}-w_{2}\right)=0, & \Gamma,
\end{array}
$$

onde $\eta_{i}$ agora representa a normal exterior a fronteira $\partial \Omega_{i}$.

Para estudarmos a dinâmica do sistema $\left(P_{\varepsilon}\right)$ introduzimos o seguinte espaço

$$
H=L^{2}\left(\Omega_{1}\right) \oplus L^{2}\left(\Omega_{2}\right) \simeq L^{2}(\Omega)
$$

com norma $\|u\|_{H}^{2}=\left\|u_{1}\right\|_{L^{2}\left(\Omega_{1}\right)}^{2}+\left\|u_{2}\right\|_{L^{2}\left(\Omega_{2}\right)}^{2}$, onde $u=\left(u_{1}, u_{2}\right), u_{i} \equiv u_{\left.\right|_{\Omega_{i}}}$. A norma em $H$ é induzida pelo produto interno $(u, v)_{H}=\sum_{i=1}^{2}\left(u_{i}, v_{i}\right)_{L^{2}\left(\Omega_{i}\right)}$. Definimos também a família de espaços de Sobolev

$$
H_{\varepsilon}^{1}=H^{1}\left(\Omega_{1}\right) \oplus H^{1}\left(\Omega_{2}\right)
$$

munido de norma

$$
\|u\|_{\varepsilon}^{2}=\left\|\nabla_{x} u\right\|_{H}^{2}+\frac{1}{\varepsilon^{2}}\left\|\nabla_{y} u\right\|_{H}^{2}+\|u\|_{H}^{2} .
$$

Agora seja $a_{\varepsilon}$ a forma bilinear sobre o espaço $H_{\varepsilon}^{1}$ dada por,

$$
\begin{aligned}
a_{\varepsilon}(u, v)=\left(\nabla_{x} u, \nabla_{x} v\right)_{H}+ & \frac{1}{\varepsilon^{2}}\left(\nabla_{y} u, \nabla_{y} v\right)_{H}+(u, v)_{H} \\
& +\varepsilon^{-1} \int_{\Gamma} k(x)\left(\gamma_{1} u_{1}(x)-\gamma_{2} u_{2}(x)\right)\left(\gamma_{1} v_{1}(x)-\gamma_{2} v_{2}(x)\right) d x
\end{aligned}
$$

onde $u=\left(u_{1}, u_{2}\right), v=\left(v_{1}, v_{2}\right) \in H_{\varepsilon}^{1}$ e $\gamma_{i}, i=1,2$, são os operadores traço definidos de $H^{1}\left(\Omega_{i}\right)$ sobre $H^{\frac{1}{2}}\left(\partial \Omega_{i}\right)$. Observando a desigualdade de Friedrich

$$
\left\|\gamma_{i} u_{i}\right\|_{L^{2}\left(\partial \Omega_{i}\right)}^{2} \leqslant \delta\left(\left\|\nabla u_{i}\right\|_{L^{2}\left(\Omega_{i}\right)}^{2}+\left\|u_{i}\right\|_{L^{2}\left(\Omega_{i}\right)}^{2}\right)
$$

que é válida para todo $u_{i} \in H^{1}\left(\Omega_{i}\right)$, e algum $\delta>0$ independente de $u_{i}$, podemos mostrar que a forma $a_{\varepsilon}$ é simétrica, contínua e satisfaz

$$
\|u\|_{\varepsilon}^{2} \leqslant a_{\varepsilon}(u, u) \leqslant C\left(1+\varepsilon^{-1}\right)\|u\|_{\varepsilon}^{2}
$$

qualquer que seja $u \in H_{\varepsilon}^{1}$, onde a constante $C$ independe de $\varepsilon$. Portanto existe um único operador $A_{\varepsilon}: D\left(A_{\varepsilon}\right) \subset H \rightarrow H$ setorial, positivo, auto-adjunto tal que

$$
a_{\varepsilon}(u, v)=\left(A_{\varepsilon} u, v\right)_{H}, \quad u \in D\left(A_{\varepsilon}\right), v \in H_{\varepsilon}^{1} .
$$


Além disso, $D\left(A_{\varepsilon}^{\frac{1}{2}}\right)=H_{\varepsilon}^{1}$ e $a_{\varepsilon}(u, u)=\left\|A_{\varepsilon}^{\frac{1}{2}} u\right\|_{H}^{2}$.

Recordemos que a aplicação $u_{i} \mapsto \gamma_{i}\left(\frac{\partial u_{i}}{\eta_{i}}\right)$ definida em $C^{1}\left(\bar{\Omega}_{i}\right)$ estende-se de modo único a um operador $\gamma_{\eta_{i}}: H^{2}\left(\Omega_{i}\right) \rightarrow H^{\frac{1}{2}}\left(\partial \Omega_{i}\right), i=1,2$. Assim entendemos que uma função $u_{i} \in H^{2}\left(\Omega_{i}\right)$ satisfaz as condições de fronteira da equação $\left(P_{\varepsilon}\right)$ se e somente se

$$
\begin{aligned}
& \gamma_{\eta_{i}} u_{i}=0, \quad \partial \Omega_{i, \varepsilon} \backslash \Gamma \\
& \gamma_{\eta_{i}} u_{i}-(-1)^{i} k(x)\left(w_{1}-w_{2}\right)=0, \quad \Gamma, \quad i=1,2 .
\end{aligned}
$$

Temos ainda que $D\left(A_{\varepsilon}\right) \subset H^{2}\left(\Omega_{1}\right) \oplus H^{2}\left(\Omega_{2}\right)$ e através de integração por partes não é difícil verificarmos que uma função $u \in H^{2}\left(\Omega_{1}\right) \oplus H^{2}\left(\Omega_{2}\right)$ satisfaz a equação $\left(P_{\varepsilon}\right)$ se e somente se $u \in D\left(A_{\varepsilon}\right)$. Além disso, das hipóteses sobre as não linearidades $f_{i}$, é claro que o operador de Nemitsküi $F_{\varepsilon}=\left(F_{\varepsilon, 1}, F_{\varepsilon, 2}\right): H_{\varepsilon}^{1} \rightarrow H$

$$
F_{\varepsilon, i}\left(u_{i}\right)(x, y)=f_{i}\left(u_{i}(x, y)\right), \quad(x, y) \in \Omega_{i}, i=1,2
$$

é continuamente Fréchet diferenciável com derivada $F_{\varepsilon}^{\prime}$ uniformemente limitada.

Teorema 6.1. Nas hipóteses acima a equação

$$
\begin{cases}\dot{u}+A_{\varepsilon} u & =F_{\varepsilon}(u) \\ u(0) & =u_{0}\end{cases}
$$

determina um semigrupo $\left\{T_{\mathcal{\varepsilon}}(t): t \geqslant 0\right\}$ atuando $H_{\varepsilon}^{1}$.

Analogamente associamos à equação $\left(P_{0}\right)$ uma equação abstrata

$$
\begin{cases}\dot{u}+A_{0} u & =F_{0}(u) \\ u(0) & =u_{0}\end{cases}
$$

no espaço $H_{s}^{1}=H^{1}(\Gamma)$, onde $D\left(A_{0}\right)=\left\{u \in H^{2}(\Gamma): \frac{\partial u}{\partial \eta}=0\right\}, A_{0} u=-\Delta u+u, u \in D\left(A_{0}\right)$ e $F_{0}$ é o operador de Nemitskǔi associado a $f=\frac{f_{1}+f_{2}}{2}$. Como no Teorema anterior a equação (6.4) determina um semigrupo $\left\{T_{0}(t): t \geqslant 0\right\}$ atuando em $H_{s}^{1}$.

Introduzimos também o espaço $L_{s}^{2}:=L^{2}(\Gamma)$ e denotamos por $(\cdot, \cdot)$ seu produto interno usual. Observamos que cada elemento $v \in H_{s}^{1}$ (resp. $v \in L_{s}^{2}$ ) estende-se naturalmente a um elemento $u \in H_{\varepsilon}^{1}$ (resp. $u \in H$ ) pela fórmula $u_{i}(x, y)=v(x),(x, y) \in \Omega_{i}, i=1,2$. Tal extensão define operadores $E_{\varepsilon} \in \mathscr{L}\left(H_{s}^{1}, H_{\varepsilon}^{1}\right)\left(\right.$ resp. $\left.E_{\varepsilon} \in \mathscr{L}\left(L_{s}^{2}, H\right)\right)$ satisfazendo para todo $v \in H_{s}^{1}$,

$$
\left\|E_{\mathcal{\varepsilon}} v\right\|_{H_{\varepsilon}^{1}}=\|v\|_{H_{s}} \quad\left(\text { resp. }\left\|E_{\varepsilon} v\right\|_{L_{s}^{2}}=\|v\|_{H}\right) .
$$

Consideremos também a projeção $M u=\left(M_{1} u_{1}, M_{2} u_{2}\right)$ definida por,

$$
M_{1} u_{1}(\cdot)=\int_{0}^{1} u_{1}(\cdot, y) d y \quad M_{2} u_{2}(\cdot)=\int_{-1}^{0} u_{2}(\cdot, y) d y .
$$


Lema 6.2. Para todo $\varepsilon \in(0,1), A_{\varepsilon}^{-1} E_{\varepsilon}=E_{\varepsilon} A_{0}^{-1}$ em $H_{s}^{1}$.

Demonstração: O operador $A_{0}$ corresponde a forma bilinear

$$
a_{0}(u, v)=\left(\nabla_{x} u, \nabla_{x} v\right)+(u, v),
$$

sobre $H_{s}^{1} \times H_{s}^{1}$. Já que para todo $u=\left(u_{1}, u_{2}\right) \in H_{\varepsilon}^{1}$ e $v \in H_{s}^{1}$

$$
\left(\nabla_{x} u, \nabla_{x} E_{\varepsilon} v\right)_{H}=\int_{\Gamma} \nabla_{x}\left(\int_{0}^{1} u_{1}(x, y) d y\right) \cdot \nabla_{x} v(x) d x+\int_{\Gamma} \nabla_{x}\left(\int_{-1}^{0} u_{2}(x, y) d y\right) \cdot \nabla_{x} v(x) d x
$$

não é difícil ver que

$$
a_{\varepsilon}\left(u, E_{\varepsilon} v\right)=a_{0}\left(M_{1} u_{1}+M_{2} u_{2}, v\right),
$$

para todo $u \in H_{\varepsilon}^{1}$ e $v \in H_{s}^{1}$. Portanto segue da identidade

$$
a_{\varepsilon}\left(u, A_{\varepsilon}^{-1} E_{\varepsilon} v\right)=\left(u, E_{\varepsilon} v\right)_{H}=\left(M_{1} u_{1}+M_{2} u_{2}, v\right)_{H_{s}^{1}}=a_{0}\left(M_{1} u_{1}+M_{2} u_{2}, A_{0}^{-1} v\right)
$$

que para todo $u \in H_{\varepsilon}^{1}$ e $v \in H_{s}^{1}$,

$$
a_{\varepsilon}\left(u, A_{\varepsilon}^{-1} E_{\varepsilon} v-E_{\varepsilon} A_{0}^{-1} v\right)=0 .
$$

Tomando $u=A_{\varepsilon}^{-1} E_{\varepsilon} v-E_{\varepsilon} A_{0}^{-1} v$ prova-se o lema.

Lema 6.3. $A$ família $A_{\varepsilon}^{-1} \stackrel{C C}{\longrightarrow} A_{0}^{-1}$.

Demonstração: Seja $\left\{f_{\varepsilon}\right\}_{\varepsilon \in(0,1]}$ uma família uniformemente limitada, isto é, $\left\|f_{\varepsilon}\right\|_{\varepsilon}=1$, colocando $u_{\varepsilon}=A_{\varepsilon}^{-1} f_{\varepsilon}$ vemos que

$$
\left(\nabla_{x} u_{\varepsilon}, \nabla_{x} u_{\varepsilon}\right)_{H}+\frac{1}{\varepsilon^{2}}\left(\nabla_{y} u_{\varepsilon}, \nabla_{y} u_{\varepsilon}\right)_{H}+\left(u_{\varepsilon}, u_{\varepsilon}\right)_{H}+\varepsilon^{-1} \int_{\Gamma} k(x)\left(\gamma_{1} u_{1, \varepsilon}(x)-\gamma_{2} u_{2, \varepsilon}(x)\right)^{2} d x=\left(f_{\varepsilon}, u_{\varepsilon}\right)_{H} .
$$

Assim

$$
\left\|u_{\varepsilon}\right\|_{H^{1}(\Omega)}^{2} \leqslant\left\|u_{\mathcal{\varepsilon}}\right\|_{\varepsilon}^{2} \leqslant\left\|f_{\varepsilon}\right\|_{\varepsilon}\left\|u_{\mathcal{\varepsilon}}\right\|_{H^{1}(\Omega)},
$$

onde $H^{1}(\Omega) \simeq H^{1}\left(\Omega_{1}\right) \oplus H^{1}\left(\Omega_{2}\right)$. Portanto $\left\|u_{\mathcal{E}}\right\|_{H^{1}(\Omega)} \leqslant 1$.

Logo, de maneira análoga a prova do Teorema 3.9 , existe $\tilde{u}_{0}:=\left(\tilde{u}_{1,0}, \tilde{u}_{2,0}\right) \in H^{1}(\Omega)$ tal que $u_{\varepsilon} \rightarrow \tilde{u}_{0}$, fracamente em $H^{1}(\Omega)$ e fortemente em $L^{2}(\Omega)$. Também de forma análoga podemos mostrar que $\nabla_{y} \tilde{u}_{0}=0$ e além disso, de $(6.5)$ vemos que $\gamma_{1} \tilde{u}_{1,0}(x)=\gamma_{2} \tilde{u}_{2,0}(x):=u_{0}(x)$ q.s em $\Gamma$. Observando que para $i=1,2$ cada $x$-seção de $\Omega_{i}$ é conexa, podemos concluir que cada $\tilde{u}_{i, 0}$ é $y$-homogênea e portanto $\tilde{u}_{i, 0}(x, y)=u_{0}(x)$ q.s em $\Omega_{i}$. Logo $\tilde{u}_{0}:=\left(u_{0}, u_{0}\right)$, com $u_{0} \in H_{s}^{1}$. Assim $A_{\varepsilon}^{-1} f_{\varepsilon} \stackrel{E}{\longrightarrow} u_{0}$ em H. Agora seguindo exatamente a prova do Teorema 3.9 obtemos que $A_{\varepsilon}^{-1} f_{\varepsilon} \stackrel{E}{\longrightarrow} A_{0}^{-1} f_{0}=u_{0}$ em $H$. 
Lema 6.4. Sejam $\varepsilon_{n} \stackrel{n \rightarrow \infty}{\longrightarrow} 0$ e $\left\{f_{\varepsilon_{n}}\right\}, f_{\varepsilon_{n}} \in H_{\mathcal{E}_{n}}^{1}$, e $f_{0} \in H_{s}^{1}$ com $f_{\varepsilon_{n}} \stackrel{E}{\longrightarrow} f_{0}$. Então se $u_{\varepsilon_{n}}=A_{\mathcal{\varepsilon}_{n}}^{-1} f_{\varepsilon_{n}}$ e $u_{0}=A_{0}^{-1} f_{0}$

$$
\lim _{n \rightarrow \infty}\left\|u_{\varepsilon_{n}}-u_{0}\right\|_{H^{1}(\Omega)}=0
$$

Demonstração: Se $u_{\varepsilon}=\left(u_{\varepsilon, 1}, u_{\varepsilon, 2}\right)$, pelo Lema anterior sabemos que $u_{\varepsilon, i} \stackrel{H^{1}(\Omega)}{\longrightarrow} u_{0}$. Logo

$$
\begin{aligned}
& 2\left\|u_{0}\right\|_{H_{s}^{1}}^{2}=2\left\|u_{0}\right\|_{H^{1}(\Omega)}^{2} \int_{\Omega}\left|\nabla_{x} u_{0}\right|^{2}+\left|u_{0}\right|^{2} \leqslant \lim \inf _{n \rightarrow \infty} \sum_{i=1}^{2}\left(\int_{\Omega}\left|\nabla_{x} u_{\varepsilon_{n}, i}\right|^{2}+\left|\nabla_{y} u_{\varepsilon_{n}, i}\right|^{2}+\left|u_{\varepsilon_{n}, i}\right|^{2}\right) \\
& \leqslant \lim \sup _{n \rightarrow \infty} \sum_{i=1}^{2}\left(\int_{\Omega}\left|\nabla_{x} u_{\varepsilon_{n}, i}\right|^{2}+\left|\nabla_{y} u_{\varepsilon_{n}, i}\right|^{2}+\left|u_{\varepsilon_{n}, i}\right|^{2}\right) \\
& \leqslant \lim _{n \rightarrow \infty}\left(\nabla_{x} u_{\varepsilon}, \nabla_{x} u_{\varepsilon}\right)_{H}+\frac{1}{\varepsilon^{2}}\left(\nabla_{y} u_{\varepsilon}, \nabla_{y} u_{\varepsilon}\right)_{H}+\left(u_{\varepsilon}, u_{\varepsilon}\right)_{H} \\
& +\varepsilon^{-1} \int_{\Gamma} k(x)\left(\gamma_{1} u_{1, \varepsilon}(x)-\gamma_{2} u_{2, \varepsilon}(x)\right)^{2} d x \\
& =\lim _{n \rightarrow \infty} \int_{\Omega} f_{\varepsilon_{n}} u_{\varepsilon_{n}, i}=2 \int_{\Omega} f_{0} u_{0}=2 \int_{\Omega}\left|\nabla_{x} u_{0}\right|^{2}+\left|u_{0}\right|^{2}=2\left\|u_{0}\right\|_{H^{1}(\Omega)}^{2} .
\end{aligned}
$$

Mostrando que $\lim _{n \rightarrow \infty}\left\|u_{\varepsilon_{n}, i}\right\|_{H^{1}(\Omega)}=\left\|u_{0}\right\|_{H^{1}(\Omega)}$.

Lema 6.5. Sejam $\varepsilon_{n} \stackrel{n \rightarrow \infty}{\longrightarrow} 0 e\left\{f_{\varepsilon_{n}}\right\}, f_{\varepsilon_{n}} \in H_{\varepsilon_{n}}^{1}$, e $f_{0} \in H_{s}^{1}$ com $f_{\mathcal{E}_{n}} \stackrel{E}{\longrightarrow} f_{0}$. Dada a caracterização variacional das soluções $u_{\varepsilon}$ e $u_{0}$ dos problemas

$$
A_{\varepsilon} u_{\varepsilon}=f_{\varepsilon} \quad e \quad A_{0} u_{0}=f_{0}
$$

sejam $\lambda_{\varepsilon}$ e $\lambda_{0}$ tais que

$$
\begin{aligned}
& \lambda_{\varepsilon}=\frac{1}{2}\left(\left\|u_{\varepsilon}\right\|_{\varepsilon}^{2}+\varepsilon^{-1} \int_{\Gamma} k(x)\left(\gamma_{1} u_{\varepsilon, 1}-\gamma_{2} u_{\varepsilon, 2}\right)^{2} d x\right)-\left(f_{\varepsilon}, u_{\varepsilon}\right)_{H} \\
& =\min _{\phi \in H_{\varepsilon}^{1}} \frac{1}{2}\left(\|\phi\|_{\varepsilon}^{2}+\varepsilon^{-1} \int_{\Gamma} k(x)\left(\gamma_{1} \phi_{1}-\gamma_{2} \phi_{2}\right)^{2} d x\right)-\left(f_{\varepsilon}, \phi\right)_{H} ; \\
& \lambda_{0}=\frac{1}{2}\left\|u_{0}\right\|_{H_{s}^{1}}^{2}-\left(f_{0}, u_{0}\right)=\min _{\phi \in H_{s}^{1}} \frac{1}{2}\|\phi\|_{H_{s}^{1}}^{2}-\left(f_{0}, \phi\right) .
\end{aligned}
$$

Então

$$
\lim _{\varepsilon \rightarrow 0} \lambda_{\varepsilon}=\lambda_{0}
$$

Demonstração: Primeiramente observemos que

$$
\lambda_{\varepsilon} \leqslant \frac{1}{2}\left\|E_{\varepsilon} u_{0}\right\|_{\varepsilon}^{2}-\left(f_{\varepsilon}, E_{\varepsilon} u_{0}\right)_{H}=\frac{1}{2}\left\|u_{0}\right\|_{H_{s}^{1}}^{2}-\left(f_{0} u_{0}\right)-\left(f_{\varepsilon}-E_{\varepsilon} f_{0}, E_{\varepsilon} u_{0}\right)_{H}=\lambda_{0}-\left(f_{\varepsilon}-E_{\varepsilon} f_{0}, E_{\varepsilon} u_{0}\right)_{H} .
$$

Logo

$$
\limsup _{\varepsilon \rightarrow 0} \lambda_{\varepsilon} \leqslant \lambda_{0}
$$


Por outro lado

$$
\begin{aligned}
\lambda_{0}= & \frac{1}{2} \int_{\Gamma}\left(\left|\nabla u_{0}\right|^{2}+\left|u_{0}\right|^{2}\right)-\int_{\Gamma} f_{0} u_{0} \\
= & \frac{1}{2} \int_{\Omega}\left(\left|\nabla_{x} u_{0}-\nabla_{x} u_{\varepsilon, i}+\nabla_{x} u_{\varepsilon, i}\right|^{2}+\left|u_{0}-u_{\varepsilon, i}+u_{\varepsilon, i}\right|^{2}\right)-\int_{\Omega} f_{0}\left(u_{0}-u_{\varepsilon, i}\right)-\int_{\Omega}\left(f_{0}-f_{\varepsilon, i}\right) u_{\varepsilon, i} \\
& -\int_{\Omega} f_{\varepsilon, i} u_{\varepsilon, i} \\
= & \frac{1}{2} \int_{\Omega}\left(\left|\nabla_{x} u_{0}-\nabla_{x} u_{\varepsilon, i}\right|^{2}+\left|u_{0}-u_{\varepsilon, i}\right|^{2}\right)+\int_{\Omega}\left(\nabla_{x} u_{0}-\nabla_{x} u_{\varepsilon, i}\right) \cdot \nabla_{x} u_{\varepsilon, i}+\frac{1}{2} \int_{\Omega}\left|\nabla_{x} u_{\varepsilon, i}\right|^{2} \\
& +\int_{\Omega}\left(u_{0}-u_{\varepsilon, i}\right) u_{\varepsilon, i}+\frac{1}{2} \int_{\Omega}\left|u_{\varepsilon, i}\right|^{2}-\int_{\Omega} f_{0}\left(u_{0}-u_{\varepsilon, i}\right)-\int_{\Omega}\left(f_{0}-f_{\varepsilon, i}\right) u_{\varepsilon, i}-\int_{\Omega} f_{\varepsilon, i} u_{\varepsilon, i} \\
\leqslant & \frac{1}{2} \| u_{0}-\left.u_{\varepsilon, i}\right|_{H^{1}(\Omega)} ^{2}+\lambda_{\varepsilon}+\int_{\Omega}\left(\nabla_{x} u_{0}-\nabla_{x} u_{\varepsilon, i}\right) \cdot \nabla_{x} u_{\varepsilon, i}+\int_{\Omega}\left(u_{0}-u_{\varepsilon, i}\right) u_{\varepsilon, i}-\int_{\Omega} f_{0}\left(u_{0}-u_{\varepsilon, i}\right) \\
& -\int_{\Omega}\left(f_{0}-f_{\varepsilon, i}\right) u_{\varepsilon, i}
\end{aligned}
$$

Lema 6.6. Sejam $\varepsilon_{n} \stackrel{n \rightarrow \infty}{\longrightarrow} 0$ e $\left\{f_{\varepsilon_{n}}\right\}, f_{\varepsilon_{n}} \in H_{\varepsilon_{n}}^{1}$, e $f_{0} \in L_{s}^{2}$ com $f_{\varepsilon_{n}} \stackrel{E}{\longrightarrow} f_{0}$. Então se $u_{\varepsilon_{n}}=A_{\varepsilon_{n}}^{-1} f_{\varepsilon_{n}}$ e $u_{0}=A_{0}^{-1} f_{0}$ com a notação do Lema anterior

$$
\lim _{n \rightarrow \infty}\left\|u_{\varepsilon_{n}, i}-u_{0}\right\|_{\varepsilon_{n}}=0, \quad i=1,2 .
$$




\section{Referências Bibliográficas}

[1] E. A. M. Abreu and A. N. Carvalho, Lower semicontinuity of attractors for parabolic problems with Dirichlet boundary conditions in varying domains, Matemática Contemporânea, 27, 37-51, 2004.

[2] F. Antoci and M. Prizzi, Reaction-diffusion equations on unbounded thin domains, Topol. Methods Nonlinear Anal. 18, no. 2, 283-302, 2001.

[3] J. M. Arrieta, Spectral properties of Schrödinger operators under perturbations of the domain, PHD thesis, Georgia Institute of Technology, Atlanta, USA, 1991.

[4] J.M. Arrieta and S.M. Bruschi, Boundary oscillations and nonlinear boundary conditions, C. R. Math. Acad. Sci. Paris 343, no. 2, 99-104, 2006.

[5] J.M. Arrieta and S.M. Bruschi, Rapidly varying boundaries in equations with nonlinear boundary conditions. The case of a Lispschitz deformation, Mathematical Models and Methods in Applied Sciences, To appear.

[6] J. M. Arrieta and A. N. Carvalho, Spectral convergence and nonlinear dynamics of reactiondiffusion equations under perturbations of the domain, Journal of Differential Equations 199, 143-178, 2004.

[7] J. M. Arrieta and A. N. Carvalho and G. Lozada-Cruz, Dynamics in dumbbell domains I. Continuity of the set of equilibria, Journal of Differential Equations, 231, no. 2, 551-597, 2006 .

[8] J. M. Arrieta and A. N. Carvalho and A. Rodríguez-Bernal, Parabolic Problems with Nonlinear Boundary Conditions and Critical Nonlinearities, Journal of Differential Equations 156, 376-406, 1999.

[9] J.M. Arrieta and A.N. Carvalho and A. Rodríguez-Bernal, Attractors for parabolic problems with nonlinear boundary bondition. Uniform bounds, Communications in Partial Differential Equations, 25 (1-2), 1-37, 2000.

[10] A.V. Babin and M.I. Vishik, Attractors of evolution equations, North-Holland, Studies in mathematics and its applications v. 25, 1992. 
[11] M.C. Carbinatto, and K.P. Rybakowski, Conley index continuation and thin domain problems, Topol. Methods Nonlinear Anal. 16, no. 2, 201-251, 2000.

[12] A. N. Carvalho and S.M. Oliva and A.L. Pereira and A. Rodríguez-Bernal, Attractors for Parabolic Problems with Nonlinear Boundary Conditions, Journal of Mathematical Analysis and Applications 207, 409-461, 1997.

[13] A.N. Carvalho and S. Piskarev, A general approximation scheme for attractors of abstract parabolic problems, Numerical Functional Analysis and Optimization 27, (7-8), 785-829, 2006.

[14] J.W. Cholewa and T. Dlotko, Global attractors in abstract parabolic problems, Cambridge University Press, London Mathematical Society lecture note series v. 278, 2000.

[15] I.D. Chueshov and A.M. Rekalo, The global attractor of a contact parabolic problem with a thin two-layer domain, Sb. Math. 195, no. 1-2, 97-119, 2004.

[16] I.S. Ciuperca, Reaction-diffusion equations on thin domains with varying order of thinness, J. Differential Equations 126, no. 2, 244-291, 1996.

[17] I.S. Ciuperca, Spectral properties of Schrödinger operators on domains with varying orders of thinness, J. Dynam. Differential Equations 10, no. 1, 73-108, 1998.

[18] R. Courant and D. Hilbert, Methods of Mathematical Physics, Interscience Publishers Inc., New York, v. 1, 1953.

[19] K. Deimling, Nonlinear Functional Analysis, Springer-Verlag, New York, 1984.

[20] T. Elsken, Limiting behavior of attractors for systems on thin domains, Hiroshima Math. J. 32, no. 3, 389-415, 2002.

[21] T. Elsken, A reaction-diffusion equation on a net-shaped thin domain, Studia Math. 165, no. 2, 159-199, 2004.

[22] T. Elsken, Attractors for reaction-diffusion equations on thin domains whose linear part is non-self-adjoint, J. Differential Equations, 206, no. 1, 94-126, 2004.

[23] T. Elsken, Continuity of attractors for net-shaped thin domains, Topol. Methods Nonlinear Anal. 26, no. 2, 315-354, 2005.

[24] T. Elsken and M. Prizzi, Characterization of the limit of some higher dimensional thin domain problems, Topol. Methods Nonlinear Anal. 20, no. 1, 151-178, 2002. 
[25] A. Friedman, Partial Differential Equations, Northwestern University, 1969.

[26] J.K. Hale, Asymptotic Behavior of Dissipative Systems, Mathematical Surveys and Monographs, v. 25, American Mathematical Society, Providence, RI, 1988.

[27] J.K. Hale and G. Raugel, Lower semicontinuity of attractors of gradient systems and applications, Ann. Mat. Pura Appl. (4) 154, 281-326, 1989.

[28] J.K. Hale and G. Raugel, Lower semicontinuity of the attractor for a singularly perturbed hyperbolic equation, J. Dynam. Differential Equations 2, no. 1, 19-67, 1990.

[29] J.K. Hale and G. Raugel, A damped hyperbolic equation on thin domains, Trans. Amer. Math. Soc. 329, no. 1, 185-219, 1992.

[30] J.K. Hale and G. Raugel, Convergence in gradient-like systems with applications to PDE, Z. Angew. Math. Phys. 43, no. 1, 63-124, 1992.

[31] J.K. Hale and G. Raugel, Reaction-diffusion equation on thin domains, J. Math. Pures Appl. (9) 71, no. 1, 33-95, 1992.

[32] J.K. Hale and G. Raugel, A reaction-diffusion equation on a thin L-shaped domain, Proc. Roy. Soc. Edinburgh Sect. A 125, no. 2, 283-327, 1995.

[33] J.K. Hale and J. Vegas, A nonlinear parabolic equation with varying domain, Arch. Rational Mech. Anal. 86, no. 2, 99-123, 1984.

[34] D. Henry, Geometric Theory of Semilinear Parabolic Equations, Lectures Notes in Mathematics, 840, Springer-Verlag, 1981.

[35] T. Kato, Perturbation Theory for Linear Operators, Grundlehren der mathematischen Wissenschaften, v. 132, Springer-Verlag, 1976.

[36] M.A. Krasnoselskii, and P.P. Zabreiko, Geometrical Methods of Nonlinear Analysis, Springer-Verlag, New York, 1984.

[37] A. Pazy, Semigroups of Linear Operators and Applications to Partial Differential Equations, Springer-Verlag, New York, v. 44, 1983.

[38] M. Prizzi and K.P. Rybakowski, Recent results on thin domain problems, Topol. Methods Nonlinear Anal. 14, no. 2, 239-255, 1999.

[39] M. Prizzi and K. P. Rybakowski, The effect of domain squeezing upon the dynamics of reaction-diffusion equations, Journal of Differential Equations 173, no. 2, 271-320, 2001. 
[40] M. Prizzi and K.P. Rybakowski, Recent results on thin domain problems. II, Topol. Methods Nonlinear Anal. 19, no. 2, 199-219, 2002.

[41] M. Prizzi and K. P. Rybakowski, Inertial manifolds on squeezed domains, J. Dynam. Differential Equations, 15, no. 1, 1-48, 2003.

[42] M. Prizzi and K.P. Rybakowski, On inertial manifolds for reaction-diffusion equations on genuinely high-dimensional thin domains 154, no.3, 254-275, 2003.

[43] M. Prizzi and M. Rinaldi and K. P. Rybakowski, Curved Thin Domains and Parabolic Equations, Studia Mathematica 151, no. 2, 109-140, 2002.

[44] G. Raugel, Dynamics of partial differential equations on thin domains, Dynamical systems (Montecatini Terme, 1994), 208-315, Lecture Notes in Math., 1609, Springer, Berlin, 1995.

[45] A.M. Rekalo, Stabilization of solutions of nonlinear parabolic equations on thin two-layer domains, Mat. Fiz. Anal. Geom. 9, no. 3, 446-454, 2002.

[46] A.M. Rekalo, Asymptotic behavior of solutions of nonlinear parabolic equations on two-layer thin domains, Nonlinear Anal. 52, no. 5, 1393-1410, 2003.

[47] R. Temam, Infinite-dimensional dynamical systems in mechanics and physics, SpringerVerlag, New York, Applied mathematical sciences v. 68, 1988.

[48] E. Sánchez-Palencia, Nonhomogeneous media and vibration theory, Lecture Notes in Physics, v. 127, Springer-Verlag, 1980.

[49] G. Vainikko, Approximative methods for nonlinear equations (two approaches to the convergence problem), Nonlinear Analysis, Methods \& Applications, 2, no 6, 647-687, 1978. 